\title{
Nucleation and Short Fatigue Crack Growth Behaviour in the 2024-T3 Aluminum Alloy
}

\author{
By \\ Jonathan P. Tsang \\ B.A.Sc. \\ A thesis submitted to the Faculty of Graduate \\ Studies and Research in partial fulfillment of the \\ requirements for the degree of \\ Master of Applied Science \\ Department of Mechanical and Aerospace \\ Engineering Ottawa-Carleton Institute for \\ Mechanical and Aerospace Engineering \\ Carleton University \\ Ottawa, Ontario \\ May 2005 \\ (C) Copyright \\ $2005 \mathrm{~J}$. Tsang
}


Library and

Archives Canada

Published Heritage Branch

395 Wellington Street Ottawa ON K1A ON4

Canada
Bibliothèque et

Archives Canada

0-494-06803-5

Direction du

Patrimoine de l'édition

395, rue Wellington

Ottawa ON K1A ON4

Canada

\author{
Your file Votre référence \\ ISBN: \\ Ourfile Notre reterence \\ ISBN:
}

\section{NOTICE:}

The author has granted a nonexclusive license allowing Library and Archives Canada to reproduce, publish, archive, preserve, conserve, communicate to the public by telecommunication or on the Internet, loan, distribute and sell theses worldwide, for commercial or noncommercial purposes, in microform, paper, electronic and/or any other formats.

The author retains copyright ownership and moral rights in this thesis. Neither the thesis nor substantial extracts from it may be printed or otherwise reproduced without the author's permission.
AVIS:

L'auteur a accordé une licence non exclusive permettant à la Bibliothèque et Archives Canada de reproduire, publier, archiver, sauvegarder, conserver, transmettre au public par télécommunication ou par l'Internet, prêter, distribuer et vendre des thèses partout dans le monde, à des fins commerciales ou autres, sur support microforme, papier, électronique et/ou autres formats.

L'auteur conserve la propriété du droit d'auteur et des droits moraux qui protège cette thèse. $\mathrm{Ni}$ la thèse ni des extraits substantiels de celle-ci ne doivent être imprimés ou autrement reproduits sans son autorisation.
In compliance with the Canadian

Privacy Act some supporting forms may have been removed from this thesis.

While these forms may be included in the document page count, their removal does not represent any loss of content from the thesis.
Conformément à la loi canadienne sur la protection de la vie privée, quelques formulaires secondaires ont été enlevés de cette thèse.

Bien que ces formulaires aient inclus dans la pagination, il n'y aura aucun contenu manquant. 
The undersigned recommends to the Faculty

of Graduate Studies and Research, acceptance of the thesis:

\title{
Nucleation and Short Fatigue Crack Growth Behaviour in the 2024-T3 Aluminum Alloy
}

\author{
Submitted by \\ Jonathan P. Tsang \\ In partial fulfillment of the requirements for \\ the Degree of Master of Applied Science.
}

Thesis Supervisor

Chairman

Department of Mechanical and Aerospace Engineering 


\begin{abstract}
In this thesis, different crack detection and monitoring techniques are studied including surface replication, marker bands, and electrical potential drop (EPD). The EPD method is the most successful short crack detection technique detecting cracks as small as $42 \mu \mathrm{m}$ in radius in the 2024 aluminum alloy.
\end{abstract}

From post-fracture analysis, it is concluded that almost all the fatigue failures originate at constituent particles, ranging from approximately $50 \mu \mathrm{m}^{2}$ to $550 \mu \mathrm{m}^{2}$ in size, with many cracks nucleating along the notch wall. This leads to the hypothesis that cracks nucleate early during fatigue testing but then become arrested. This process repeats until one crack breaks through the arresting barrier and propagates to failure. The time required for the crack to break through barrier is the reason why the majority of the fatigue life is spent in the nucleation and short crack regimes.

A chemical analysis of the constituent particles reveals a compositional difference between the large particles at the nucleation sites and the smaller particles that have no role in fatigue crack nucleation. The larger particles contain significant iron and manganese and increased copper compared to the smaller particles. Therefore, to improve the fatigue life of 2024 , the iron content must be lowered. 


\section{ACKNOWLEDGEMENTS}

First and foremost, I would like to thank my mother, Ivy Wong, for all of her encouragement, guidance and love. She was always there for me when I needed help or advice and her strength and compassion inspires me to do my best. Without her, none of this would have been possible.

I'd also like to thank Dr. Jonathan Beddoes and Dr. Ali Merati for their helpful supervision. They have always been there to help with any technical problems and were also able to give me enough space and responsibility so that I may exercise my own judgment. Both of them have always had my best interest in mind and a graduate student could not ask for better supervisors.

Dr. Rick Kearsey and Scott Yandt have provided a lot of help and consultation for my thesis work, especially in regards to the EPD testing. It has been a tremendous benefit to have them work so closely with me. Thanks also go to Dr. Stephen Smith at NASA for his help with the EPD testing.

Lastly, I'd like to thank the Department of National Defence for partial funding of this thesis work. 


\section{TABLE OF CONTENTS}

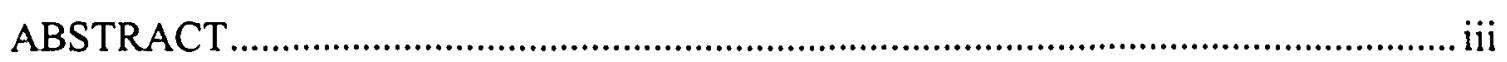

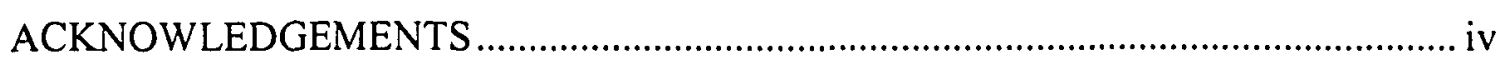

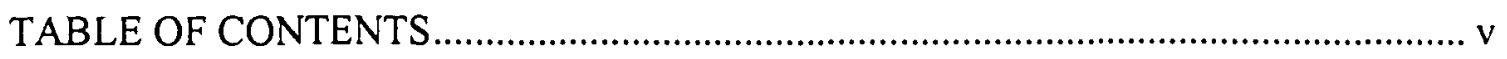

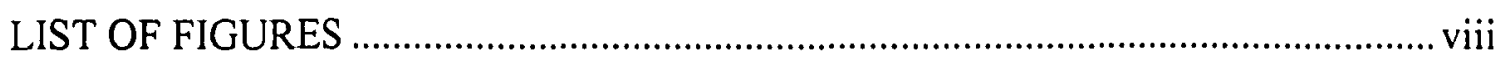

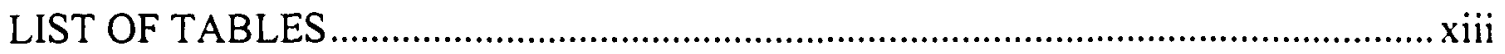

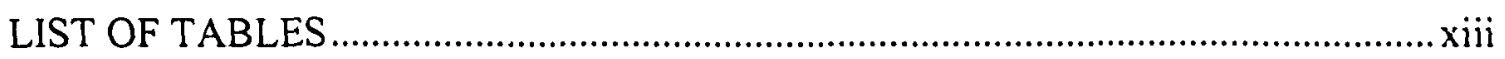

1.0 INTRODUCTION

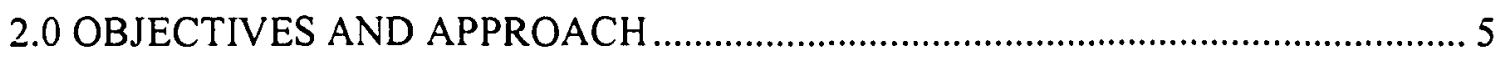

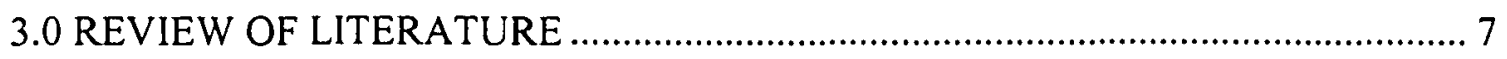

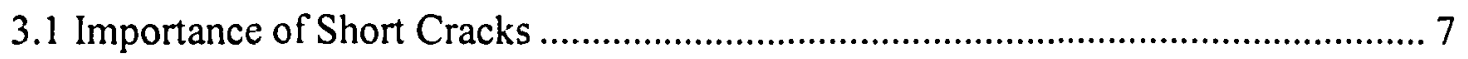

3.2 Difference Between Short and Long Cracks ..................................................... 8

3.3 Fatigue Crack Initiation and Short Crack Growth Behaviour .............................. 12

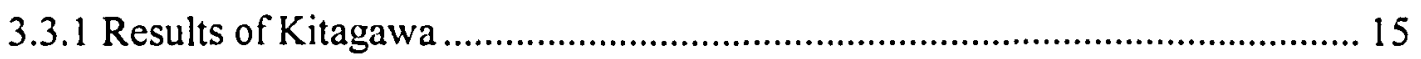

3.3.2 Results of Hunter and Fricke ……………................................................. 16

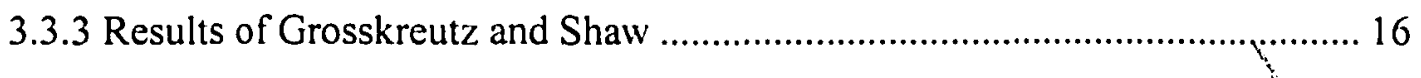

3.3.4 Results of Kung and Fine ............................................................................. 17

3.3.5 Results of the Australian Defence Science and Technology Organization

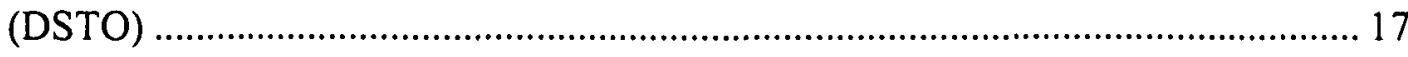

3.4 Characterization of Crack Nucleation Sites ....................................................... 19

3.4.1 Equivalent Initial Flaw Size (EIFS) ....................................................... 19

3.4.2 Equivalent Pre-crack Size (EPS) ……………………............................. 21 


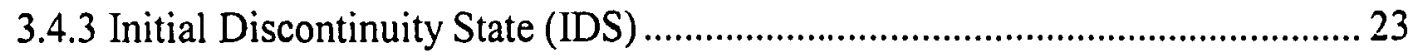

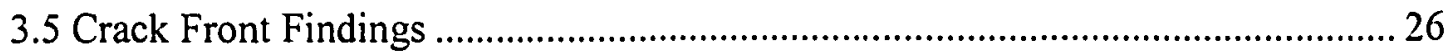

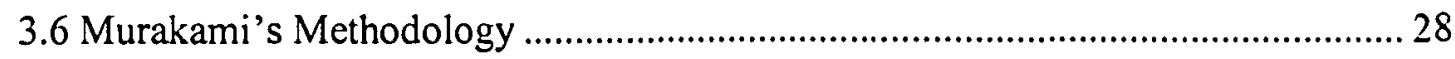

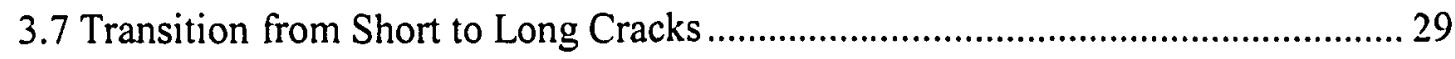

3.8 Energy Dispersive X-ray (EDX) Analysis........................................................... 32

3.9 Electrical Potential Drop (EPD) Technique............................................................ 36

3.9.1 Use of Electrical Potential Difference (EPD) for Measuring Crack Size........ 38

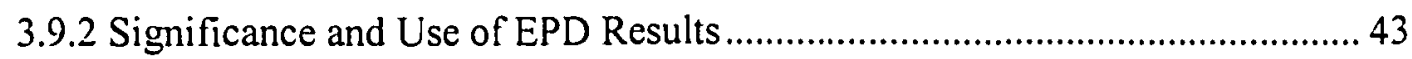

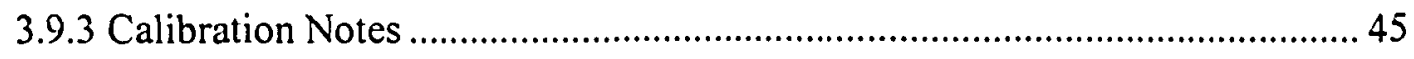

4.0 EXPERIMENTAL PROCEDURES AND MATERIALS …………...................... 48

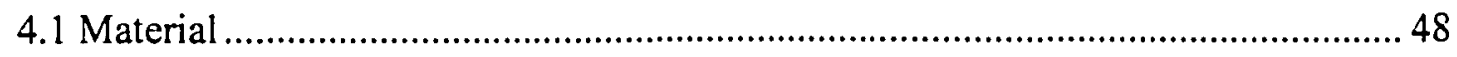

4.2 Procedures

4.2.1 Metallography and Image Analysis ........................................................... 52

4.2.2 Microsoft Excel Analysis Procedure ………………...................................... 55

4.2.3 Fatigue Testing Procedure ………………………................................... 56

4.2.4 Surface Replication ................................................................................ 57

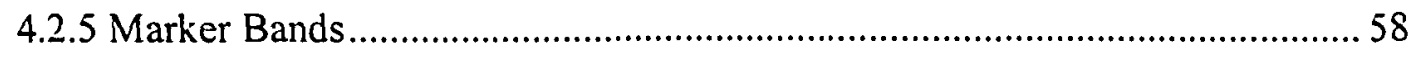

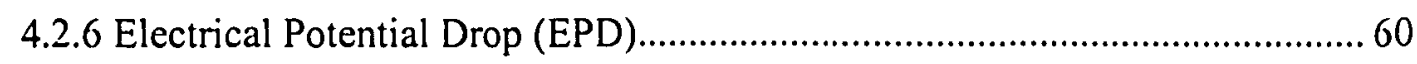

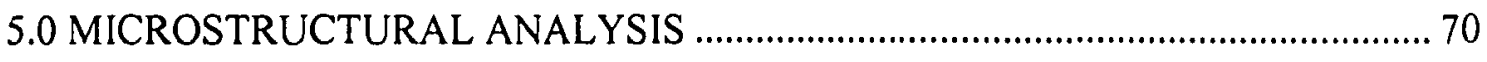

5.1 Metallographic Thickness Analysis.................................................................. 71

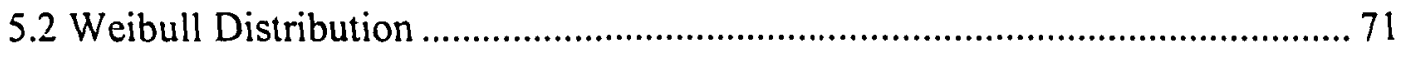

5.3 Microstructural Analysis...................................................................... 72

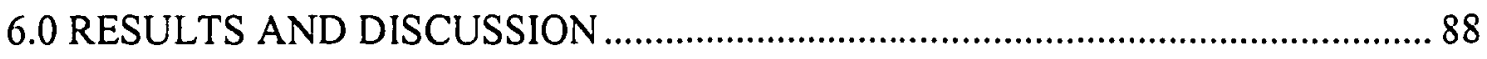




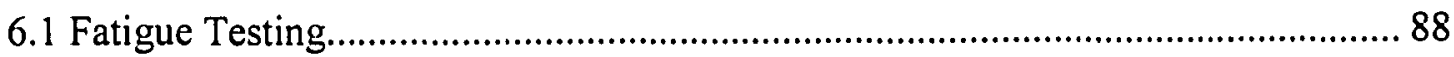

6.2 Surface Replication Technique ......................................................................... 93

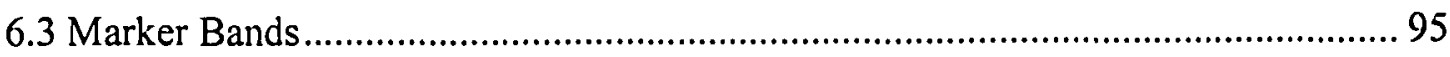

6.4 Electrical Potential Drop (EPD)...................................................................... 98

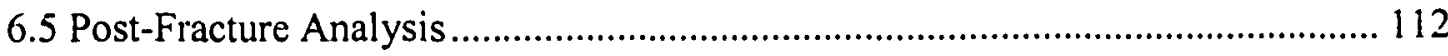

6.6 Energy Dispersive X-ray (EDX) Analysis........................................................ 126

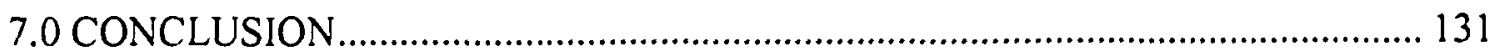

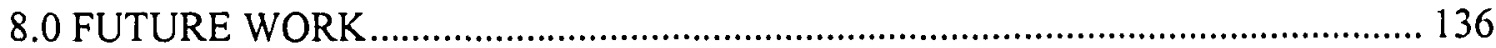

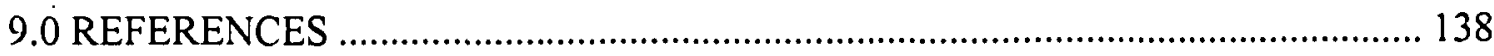

APPENDIX A - Image Pro Procedure ...................................................................... 144

APPENDIX B - Microsoft Excel Analysis Procedure .................................................... 159

APPENDIX C - Specimen Preparation and Loading Procedure.................................... 173

APPENDIX D - EPD Testing Procedure ............................................................... 198 


\section{LIST OF FIGURES}

Figure 1. da/dN vs. a curve showing the transition between short and long cracks [11] 31

Figure 2. Cross-section of the $\mathrm{Si}-\mathrm{Li}$ detector [33] 33

Figure 3. The $\mathrm{x}$-ray detection and photoelectron creation process [33] …....................... 34

Figure 4. Schematic of the EDS system [33] ............................................................... 34

Figure 5. EDX sorting of characteristic x-ray photon data. Each column would represent a certain element with those certain $\mathrm{x}$-ray energy characteristics. In this example, the EDX resolution is $100 \mathrm{eV}$

Figure 6. Influence of different potential lead locations on measured potential differences $[35]$ .46

Figure 7. Influence of different current lead locations on measured potential drops [35]47

Figure 8. A typical example of the image analysis performed on the SEM micrographs using the ImagePro Software.

Figure 9. Schematic showing the location of pictures taken during metallography. For the LS and ST planes, the locations were chosen such that a through-thickness microstructural analysis was performed. Ten rows of ten pictures were taken in each plane for a total examination area of over $2 \mathrm{~mm}^{2}$. .55

Figure 10. Single-edge notched (SEN) fatigue sample................................................. 56

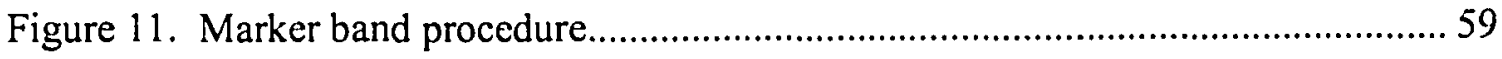

Figure 12. Wiring diagram for EPD crack measurements ............................................ 61

Figure 13. Voltage drop reading stability test at 10A of current .....................................64 64

Figure 14. Voltage drop reading stability test at $12 \mathrm{~A}$ of current ...................................... 65 
Figure 15. Voltage drop reading stability test at $14 \mathrm{~A}$ of current

Figure 16. Voltage variation test with an amplification of the voltage signal by $5000 \times .67$

Figure 17. Voltage variation test with an amplification of the voltage signal by $10000 x$

Figure 18. Three dimensional microstructure of the new, bare 2024-T3 aluminum alloy, using a) optical microscopy and b) scanning electron microscopy (backscattered electrons)

Figure 19. Typical backscattered SEM micrographs of the 2024-T3 aluminum alloy showing the a) LT plane, b) LS plane and c) ST plane.

Figure 20. Comparison of the average physical characteristics of the constituent particles throughout the thickness in the LS Plane. 77

Figure 21. Comparison of the average physical characteristics of the constituent particles throughout the thickness in the ST Plane. 77

Figure 22. Coordinates of all particles in the LS plane over a) $0.5 \mu \mathrm{m}^{2}$, b) $25 \mu \mathrm{m}^{2}$, c) 75 $\mu \mathrm{m}^{2}$, and d) $100 \mu \mathrm{m}^{2}$. 78

Figure 23. Coordinates of all particles in the ST plane over a) $0.5 \mu \mathrm{m}^{2}$, b) $25 \mu \mathrm{m}^{2}$, c) 75 $\mu \mathrm{m}^{2}$, and d) $100 \mu \mathrm{m}^{2}$. 79

Figure 24. Linearity plot for the Weibull Distribution of the area of the particles in the LT plane of 2024-T3 80

Figure 25. Linearity plot for the Weibull Distribution of the area of the particles in the LS plane of 2024-T3. .81

Figure 26. Linearity plot for the Weibull Distribution of the area of the particles in the ST plane of 2024-T3. 82 
Figure 27. Cumulative Weibull Distributions of the area of the constituent particles in the LT, LS, and ST planes. 83

Figure 28. The Distribution of the area of the constituent particles in the LT plane....... 84 Figure 29. The Distribution of the area of the constituent particles in the LS plane....... 85

Figure 30. The Distribution of the area of the constituent particles in the ST plane....... 86 Figure 31. Average dimensions of the constituent particles in the LT, LS and ST planes 87

Figure 32. Fatigue results for only the specimens that were tested until failure 92

Figure 33. Optical micrograph of smallest crack found with the surface replication technique 94

Figure 34. Typical set of marker bands found during optical microscopy... 95

Figure 35. Typical fracture surface with identifiable marker bands shown with the corresponding cycle count. 96

Figure 36. DCPD data for 2024-DCPD-TK-87 99

Figure 37. DCPD and marker band data for 2024-DCPD-TK-87 100

Figure 38. Typical EPD data plot for a sample tested with 12A and 5000X gain (2024DCPD-TK-52) 102

Figure 39. This is a SEM micrograph of the above sample showing the $1.3 \mathrm{~nm}$ crack detected by EPD method. 103

Figure 40. Static voltage stability test using $12 \mathrm{~A}$ of current and $10000 \mathrm{X}$ gain ........... 104

Figure 41. EPD voltage drop data for sample TK-59 ............................................... 105

Figure 42. EPD voltage drop data for sample TK-60 ................................................. 106

Figure 43. Averaged EPD data from sample TK-57 ................................................... 107 
Figure 44. Fracture surface of sample TK-57 showing $250 \mu \mathrm{m}$ radii crack found during fatigue testing 108

Figure 45. Averaged EPD data from sample TK-65 110

Figure 46. Fracture surface of sample TK-65 showing a) $42 \mu \mathrm{m}$ radii crack found during fatigue testing and $b$ ) the same crack using the backscattered electron detector to show the size of the constituent particle in relation to the crack size. 110

Figure 47. Typical crack nucleation sites found using the backscattered electron detector in the SEM 114

Figure 48. Particle area distribution in the ST plane with the nucleating particle data included

Figure 49. Examples of different cracks found along the notch wall taken with the a) secondary electron detector, b) backscattered electron detector, and also c) \& d) at high magnifications. Micrographs in e) and f) are taken at the notch wall of a sample that was fatigue for $\sim 17500$ cycles, showing no cracking at the constituent particles. 119

Figure 50. Crack growth rate vs. crack length [7] 121

Figure 51. Schematic of proposed hypothesis on the notch wall 122

Figure 52. Crack nucleation and growth hypothesis of a crack that eventually leads to failure 123

Figure 53. Chemical EDX analysis of the 2024-T3 aluminum matrix 127

Figure 54. Typical EDX analysis of small particles found in metallography 128

Figure 55. Typical EDX analysis of large particles found during metallography 129 
Figure 56. Typical EDX analysis of large particles found at the nucleation site on the

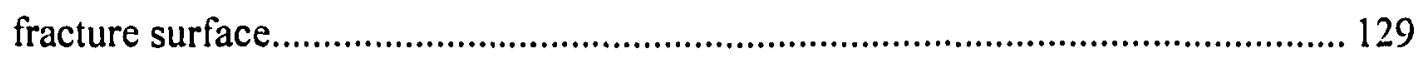




\section{LIST OF TABLES}

Table 1. Composition of AA2024 [36] ................................................................... 51

Table 2. Minimum mechanical properties of AA2024-T3 in the longitudinal (rolling)

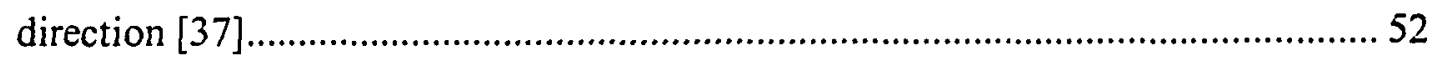

Table 3. Composition of Keller's reagent ………....................................................... 53

Table 4. Ranges for the measurement of different physical properties of the constituent particles

Table 5. Data collected from metallographic analysis of constituent particles 73

Table 6. Dimensions of particles in the L, T, and S directions...................................... 74

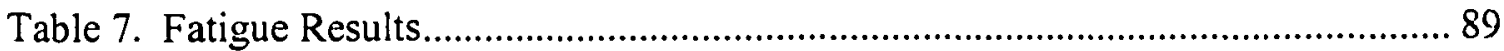

Table 8. Constituent particle data collected from the crack nucleation region.............. 115

Table 9. Approximate average chemical composition of each phase examined during the EDX analysis 130 


\subsection{INTRODUCTION}

Starting in the mid-1950's, aircrafts were designed using the safe-life approach where a particular structure was designed to operate for a certain number of in-service hours without significant fatigue damage occurring. The in-service hours or safe lives were chosen and substantiated based on four full-scale fatigue tests [1]. A major drawback to the safe-life approach was that it did not adequately account for possible initial manufacturing flaws that may exist in the airframe at delivery. Studies performed in the early 1970 's showed that over $50 \%$ of fatigue failures nucleated at initial flaws induced during manufacturing [1]. As a result, the safe-life approach was abandoned.

Another reliability approach that was used in the design of aircraft was the fail-safe approach. This approach requires that the structure be capable of sustaining complete failure or obvious partial failure of a single principal structural element at fail safe loads

[1]. As a result, aircraft designed with the fail safe approach had secondary load paths built into the design of the structure so that if any part of the structure failed, the load was carried through a secondary path. The fail-safe approach was thought to be the solution to the structural fatigue problems. However, there were concerns about the loss of failsafety with age and this was realized by the loss of an AVRO 748 in April 1976 due to multiple-site fatigue damage in the wing. In May 1977, a Dan-Air 707 aircraft lost a horizontal stabilizer, which was designed with the fail-safe approach, because of fatigue. As a result, the commercial transport industry lost faith in the fail-safe approach and 
introduced damage tolerance principles in December 1978. Since then, all commercial aircraft have been designed with a damage tolerant philosophy [1].

The damage tolerance approach (DTA) differed from the safe-life and fail-safe approach in that the method assumes the presence of defects or flaws that may have resulted from manufacturing. Also, the DTA predicts lifetimes based on fatigue crack growth data and fracture mechanics principles rather than statistical analysis of low-cycle fatigue tests used for the safe-life and fail-safe approaches. Based on the current knowledge of the crack growth rates, non-destructive inspection's probability of detection, and limiting crack size when a crack becomes unstable, a safe inspection interval is established such that if a crack is missed during the inspection, it will not become unstable before the next inspection. If during the inspection, no cracks are detected, the structure is returned to service for another safe inspection interval. This process is repeated until a crack is detected and the structure is either retired or it is repaired and returned to service. The DTA, much like the fail-safe approach, was thought to be the solution for structural fatigue problems. However, a 737 Aloha Airlines aircraft suffered a fatal accident due to a pressurized fuselage fatigue failure. Undetected multi-site fatigue damage in the critical rivet row of a skin splice caused skin crack coalescence and un-arrested fast fracture [1].

As can be seen, all three design philosophies have shown to be inadequate and one of the most important issues that need to be addressed are the effects of multi-site, small crack damage on residual strength, especially on aircraft who have exceeded half of their 
designed service life. A lot of the aircraft used today have exceeded their safe-life and remain in service, after adopting a damage tolerant operation approach, due to economic factors. Of these aircraft, almost all of the older large civilian passenger aircrafts and in certain military transport aircrafts, such as the Hercules C-130, used 2024-T3 for the fuselage and lower wing skins [2]. As a result, life prediction models have grown in importance for materials such as 2024-T3, which are used abundantly in aging aircraft, and because of the inadequacies of the fracture mechanics-based models used in the DTA approach, there is currently a push to replace these models with a holistic life prediction methodology. A holistic-based life prediction methodology is becoming more popular because it is material-based and encompasses the entire fatigue life, from the very beginning until failure. The advantage of such a methodology is that a complete and material-based model will be adaptive to other types of damage, environments and conditions.

The fracture mechanics-based methodologies do not accurately model the nucleation and growth of short cracks, which can play a large role in fatigue failures especially in regards to multi-site, small crack damage. Instead, these models utilize long crack data obtained from compact tension testing and linear elastic fracture mechanics (LEFM) to back-calculate the theoretical initial flaw size. However, pre-cursor work [3][4][5] involving post-fracture analysis of fatigue coupons and metallurgical studies has shown that the sole microstructural discontinuity controlling crack nucleation for high cycle fatigue failures is secondary phase constituent particles in 2024-T3. It is also observed that the nucleation and short crack growth made up about $95 \%$ of the fatigue life and that 
the constituent particle sizes were in the range of the short crack regime [3][4][5]. The short crack regime, which typically extends over 2-3 grains in size, exhibits different characteristics than the long crack regime. This is a result of the fact that linear fracture mechanic principles do not apply to cracks that are in the range of short cracks. Instead, other physical features of the material play an important role in the crack growth and behaviour by affecting the local stress field in front of the crack tip. These features include grain size, grain boundaries, inter-particle spacing, and texture.

In addition, it was observed in a previous study [3][4][5] that no correlation could be found between the nucleation size and the fatigue life, indicating that short cracks are the dominant regime in the fatigue life of high strength aluminum alloys and must be studied accurately before an accurate holistic model can be achieved. 


\subsection{OBJECTIVES AND APPROACH}

A five-stage experimental program will be used to study the nucleation and short fatigue crack growth behaviour in the candidate material. The objectives of this program are to:

a) Identify and develop a suitable technique for detection and monitoring of crack growth in the nucleation and short crack regimes.

b) Develop a better understanding of early fatigue crack growth in the 2024-T3 aluminum alloy and compare it with long crack growth.

The observations and data obtained from this thesis will be used to develop a more accurate methodology for an estimate of the fatigue life of aluminum aircraft components. In particular, this will add a greater quantitative and physical understanding of nucleation and short crack growth behaviour to current life prediction models that are dominated by extensive knowledge of long cracks.

This thesis research comprises five stages. The approach of each stage will be as follows:

i) Stage 1-Literature Review. An extensive literature review of the microstructural factors that influence nucleation and short crack behaviour, and different short crack detection methods. (Chapter 3) 
ii) Stage 2-Metallography. A microstructural and metallographic analysis was performed to acquire physical descriptions of the inherent discontinuities of 2024T3, known as the Initial Discontinuity State (IDS). (Chapter 5)

iii) Stage 3-Fatigue Testing. Fatigue tests were performed on single edge notched tensile (SENT) samples. (Chapter 6.1)

iv) Stage 4-Development of Crack Monitoring Techniques. Surface replication, marker bands, and electrical potential drop (EPD) crack detection techniques were evaluated and compared to identify and develop a capable technique for detecting and monitoring cracks in the nucleation and short crack regimes. (Chapter $6.2-$ 6.4)

v) Stage 5-Post-Fracture Analysis. A study of the mechanisms of early crack growth and to determine the correlation between the IDS and the failure origins of cyclic damage through examination of the fracture surface using a scanning electron microscope (SEM). In addition, a limited compositional analysis was performed on the constituent particles using an energy dispersive $\mathrm{x}$-ray (EDX) system. (Chapter $6.4-6.5$ ) 


\subsection{REVIEW OF LITERATURE}

In 1975, Pearson observed that small, naturally-initiated cracks in BS L65 and DTD 5050 , two commercially available aluminum alloys, did not behave according to long crack growth data that was acquired [6]. His discovery has now spanned almost three decades of investigation into the short crack phenomena. During this time, the study of cracking in aluminum alloys has led to three distinct stages of cracks [7]. These three types of fatigue cracks are:

- microstructurally small fatigue cracks whose length is in the range of a microstructural feature such as a single grain,

- physically small fatigue cracks whose crack length is typically between $50-500$ $\mu \mathrm{m}$, and

- long cracks which are greater than $0.5 \mathrm{~mm}$ in length.

\subsection{Importance of Short Cracks}

Most studies on fatigue performance have concentrated on long cracks. However, it has been observed that nucleation and short fatigue cracks dominate the fatigue performance of an aluminum alloy. In fact, Merati et al. [3][4][5] have shown that nucleation and short crack growth makes up to $95 \%$ of the total fatigue life. The study and understanding of the short crack regime is important because in this same study, it was 
shown that the initial flaw size back-calculated from a life prediction model, which uses long crack growth rate data as an approximation, does not match the measured initial flaw size found on the fracture surface. In addition, there was no correlation found between the initial flaw size on the fracture surface and the fatigue performance of the same alloy. A larger initial flaw or discontinuity did not translate into a lower fatigue life and a smaller discontinuity did not result in a longer fatigue life. As a result, the size of the nucleation feature hardly affects the fatigue life and can be considered to have almost negligible influence. That means that the majority of the fatigue life is spent in the short crack regime and it plays the most significant role in fatigue life prediction methodologies and must be fully understood in order to provide an accurate holistic life prediction.

Also, it has been the assumption that small defects, which exist in the material, will not experience crack growth and ultimately, will not experience fatigue failure provided the stress intensity factor is less than the threshold stress intensity factor for long crack growth, $\Delta \mathrm{K}_{\mathrm{th}}$. This assumption is known to be false in the short crack regime [8] and it further emphasizes the importance of understanding short cracks and using proper short crack data rather than assumptions and estimations based on long cracks.

\subsection{Difference Between Short and Long Cracks}

When dealing with cracks, crack growth rate, and predicting fatigue lives, most investigators have used the linear elastic fracture mechanics (LEFM) approach [8]. The 
LEFM approach has also been used to encompass different materials (i.e. steels, aluminum alloys, titanium alloys, etc) and for different testing parameters and specimen configurations (i.e. constant amplitude loading, variable amplitude loading, notches, holes, corrosion damage, etc). In this approach, the change in the stress intensity factor, $\Delta \mathrm{K}$, is usually used to predict the crack growth rates $(\mathrm{da} / \mathrm{dN})$ through the use of ParisLaw type descriptions.

Short cracks grow quicker than predicted by traditional da/dN vs. $\Delta \mathrm{K}$ data and do not follow conventional linear elastic fracture mechanics because short cracks exhibit anomalous growth $[7][8][9][10]$. The anomalous behaviour of short cracks can be linked to microstructural interactions, which can include the influence of grain boundaries on crack tip plasticity and crack direction, or the alteration of crack closure conditions caused by crack path or crack surface changes.

Crack closure plays a significant role on long crack growth rates and it effectively reduces the driving force for crack propagation. In the long crack regime, a region of plastically deformed material forms ahead of the crack tip because the highly concentrated stress in this region exceeds the yield strength of the material. As the crack continues to propagate, a wake of plastically deformed material develops around the crack. This plastic wake is basically a region of permanently stretched/deformed material in a field of elastic material. As a result, when the tensile load is reduced in a tensiontension cyclic fatigue test, the faces of the crack actually come in contact, even when the load is above zero. An increased tensile load must now be applied in order to fully open 
the crack tip and since crack propagation only occurs when the crack tip is open, the effective force for crack growth is now reduced from $K_{\max }$ to $\mathrm{K}_{\max }-\mathrm{K}_{\mathrm{cl}}$ where $\mathrm{K}_{\mathrm{cl}}$ is the driving force required to open the crack tip. Short cracks do not experience such large scale wakes because the crack size is too small relative to the crack tip plastic zone size to allow constraint of the zone, and as a result, do not experience the same amount of crack closure effect. The reduced amount of crack closure effect (i.e. higher driving force for crack propagation) may significantly contribute to the increased growth rate of short cracks.

Similarly, the LEFM methodology also fails because it is based on the assumption of small scale yielding in front of the crack tip. Some [11] have suggested that the reasonable limit for the applicability of LEFM is that the plastic zone size must be less than $1 / 5$ of the crack size. Since most short crack tests are conducted at high stress levels $\left(>0.5 \sigma_{y s}\right)$, significant amounts of plasticity occurs in front of the crack tip and the small scale yielding assumption is not valid in the short crack regime.

Another reason why short cracks propagate more rapidly than long cracks is because, for small cracks, similitude breaks down and the crack growth rate is no longer a unique function of only the stress intensity factor [9]. Metallurgical similitude is a condition in which the crack front encounters a large number of microstructural units (grains in this particular case) and the continuous crack growth rate is averaged over a large number of favourable and unfavourable grains. When the crack length is only on the order of a couple of grains, then the plastic zone deformation is greatly influenced by local 
microstructure because plastic deformation at the crack tip is assumed to occur as one or two slip bands that are inclined in the direction of the applied load and are confined. Acceleration and deceleration of the crack growth rate is a result of the interaction between the plastic zone crack tip with barriers to plastic deformation such as grain boundaries [9].

At the outset, when the crack length is less than a grain size or barrier spacing, the crack grows at an accelerated pace. As the crack approaches the grain boundary or barrier, the crack growth rate decreases and comes to arrest if the barrier is strong or if the slip systems in the neighbouring grain are unfavorably oriented [7]. Otherwise, a favorably oriented slip system in the neighbouring grain will not hinder or arrest the crack and the crack will continue to grow until it reaches the next barrier. As a result, it is important to note that the retardation of the crack does not necessarily have to occur at the first grain boundary or barrier and that the crack growth rate versus crack length varies significantly from grain to grain. It is not until the transition from short crack to long crack that the variability in growth rates become negligible [12]. This non-continuous crack growth pattern in the short crack regime, as mentioned previously, leads to the failure of LEFM assumptions.

However, an important consideration when dealing with the nucleation and short growth of fatigue cracks is the stress or strain level applied to testing or application. Plumtree and O'Connor [13] concluded that the microstructural features, which impaired crack growth, were dependent on the stress or strain level. This was later confirmed by Li [11], 
who observed that initiation and early crack growth was also dependent on applied stress and strain levels during fatigue testing. At high strains, it was found that the grain boundaries and secondary phase particles were traversed easily and, as a result, particle de-cohesion and cracking were observed. At low strains, the grain boundaries and constituent particles are effective barriers to early crack growth and only de-cohesion between the particle and the matrix was observed. Triple points and highly misorientated boundaries represented the greatest impediments to crack growth.

\subsection{Fatigue Crack Initiation and Short Crack Growth Behaviour}

In commercial Al-alloys, the nucleation of fatigue cracks is generally associated with constituent particles, whereas, in most steels and Al-bronze alloys, inclusions/inclusion clusters are the most significant microstructural feature. For ferrite, $\mathrm{Cu}$, and $\mathrm{Ni}$-alloys, slip band cracking is generally the microstructural discontinuity associated with fatigue cracks. Intermetallic particles imbedded in the matrix act as nucleation sites because they will affect the local, microscopic stress concentration field and act as stress raisers that facilitate cracking.

It has been shown in many research papers $[7][8][9][10]$ that short cracks can nucleate and propagate at stress levels below the fatigue threshold as predicted by the $\mathrm{da} / \mathrm{dN}$ curve for long cracks. As a result, short cracks must follow different conditions for crack growth than long cracks. The following criteria were found for rapid growth of short cracks: 
- ratio of plastic zone size to surface crack length $>0.05$, and

- ratio of plastic zone size to size of controlling microstructure (i.e. grain size) $<1$.

Currently, there is a debate as to whether cracks start at the interface between the particle and the matrix, as a crack in the particle, or at a slip band impinging on a particle. Merati et al. [3][4][5] conducted a study prior to this thesis that shows that the constituent particles found at the nucleation site were, in most cases, cracked. It is believed that the cracking within particle may already be there from processing such as rolling where the larger particles are crushed into particle clusters [14].

An accurate and effective quantitative method for evaluating the effects of constituents and small defects has not been developed because of the complication of the dominant mechanism and constituent configurations during crack nucleation. It is known, however, that the effect of the smaller constituents depends on their location (i.e. the surface of the material where crack nucleation is generally found/associated with because at the surface, the restraint on cycle slip is relatively low). These effects are a direct result of the stress concentration field normally found surrounding the defect or constituent. The effect of constituents or small defects is a short crack problem because the size of these constituents and defects are in the range of short cracks and therefore, they should be regarded as short cracks [3][4][5]. 
As expected, the time interval for a crack tip to be arrested at a grain boundary decreases as crack length increases until it reaches a point in the long crack regime that the arrest time at a grain boundary is negligible. Because of such significant dependence and interaction with the local microstructural features that are randomly distributed, the stress state and crack length are not sufficient to describe short cracks. Therefore, numerical analyses were used to investigate the short crack phenomenon. Akiniwa et al. [15] concluded that the probability distribution of the crack propagation rate was approximated by a three-parameter Weibull distribution, which Goto [16] also later used to analyze the distribution of crack initiation life, propagation life, fatigue life, and crack length.

Akiniwa et al. [15] then proposed a Monte Carlo simulation model, which simulated the short fatigue crack growth rates by assuming that the crack growth rates were dependent on the crack tip opening displacement (CTOD) shown by

$$
\frac{d a}{d N}=C(\triangle C T O D)^{n}
$$

In this simulation, a continuous distribution of dislocations ahead of the crack tip was assumed and it was also conducted by having the grain size, friction stress within each grain, and stress intensity required for slip across a grain boundary as random variables that follow a two-parameter Weibull distribution. In this model, a fatigue crack was assumed to have started from the weakest grain, which had the highest value of grain size diameter multiplied by the effective stress $\left(\Delta \sigma-2 \sigma_{f}\right)$ and the amount of crack 
propagation over a certain number of cycles $(\Delta \mathrm{N})$ was then calculated from equation [1] by assuming $\mathrm{C}=1$ and it was kept smaller than one-tenth of grain size by adjusting $\Delta \mathrm{N}$ in the simulation.

\subsubsection{Results of Kitagawa}

Kitagawa et al. [17] observed that several cracks initiate along the wall of the notch on a plane perpendicular to the loading axis and continue growing until they connect with another crack forming one crack plane. They also observed that when the crack reaches about $170 \mu \mathrm{m}$ in crack length ( $2 \mathrm{a}$ for surface cracks), the crack front becomes semicircular and remains so until $b / t \approx 0.4-0.5$, where $b$ is the maximum depth of the crack and $t$ is the thickness of the specimen. Subsequently, the crack front becomes semielliptical with the major axis along the surface of the specimen. Similar results were obtained at different stress levels, except that there were shape differences in the later stage of the fracture process, depending on the stress level.

Kitagawa et al. [17] used fatigue samples with an external initial flaw size prepared by an arc-strike on the surface of the specimen. A couple of methods were employed to detect and monitor crack growth. One of the methods was a "crack surface colouring technique," where cracks would be detected on the surface by heat treatment oxidation. The other methods were to use a surface replication method and by using "marker bands." Marker bands are applied by varying the stress levels so that there was a set number of underload and nominal load stress cycles. 


\subsubsection{Results of Hunter and Fricke}

Hunter and Fricke [18][19], in their examination of the 2024-T3 Al alloy, were not able to establish a relationship between fatigue crack initiation and slip bands. However, they still proposed that these cracks did initiate on coarse slip bands. They also believed that the constituent particles did not act as "focal points" for crack initiation and rather, cracks would detour around these constituent particles. If a void or notch were associated with the constituent particle, then this would act as a "focal point" for crack initiation and advancement.

\subsubsection{Results of Grosskreutz and Shaw}

Conversely, Grosskreutz and Shaw [20] found that all fatigue cracks in 2024-T4 Al alloys initiated at constituent particles. Very fine slip lines were observed in grains, even though the nominal applied stress was less than half of the yield stress. For most of the constituent particles, it was noticed that the region adjacent to the constituent particle had a higher concentration of these fine slip lines, although it does not appear that this had an effect on crack initiation. All of the fatigue cracks were found to have initiated near particles that did not have the higher concentration of slip line regions. Grosskreutz and Shaw suggested that the cracks would initiate at the boundary between the matrix and the constituent particle through interface de-bonding. 


\subsubsection{Results of Kung and Fine}

Kung and Fine [21] performed a crack initiation and micro-crack growth study of 2024T4 and 2124-T4. The 2124 alloy is a higher purity version of 2024 containing less constituent particles and larger grain sizes. It was found during this study that all of the fatigue cracks in the 2024 alloy and half of the cracks in the 2124 alloy were nucleated from constituent particles. However, the fatigue performance of the 2124 alloy was just slightly better than the fatigue life of the 2024 alloy. This finding suggests that the advantage of the 2124 alloy being purer and having less constituent particles was somewhat offset from the disadvantage of the larger grain size.

They observed two modes of fatigue crack initiation. In the first mode, crack initiation occurred on coarse slip bands that were not associated with constituent particles or polishing pits. These cracks were found to have formed within the interior of the grain, away from the grain boundary. The other mode of crack initiation was found to be associated with constituent particles or pits.

\subsubsection{Results of the Australian Defence Science and Technology Organization (DSTO)}

On samples with the as machined surface or polished with abrasive paper, Newman et al. [22][23] found that fatigue cracks initiated from constituent particles or constituent particle clusters (typically $3 \mu \mathrm{m}$ wide and $12 \mu \mathrm{m}$ deep) in thin 2024-T3 sheets. However, 
Barter et al. [9], in their work with the DSTO, found that the initiating particles were slightly larger than the $12 \mu \mathrm{m}$ that Newman [22][23] had first noted.

In addition, from their study of the initiation and early growth of fatigue cracks in 7050T7451 [9], some of the initiating constituent particle clusters were found to not intersect the surface. In those cases, however, they were generally very close (within $10 \mu \mathrm{m}$ ) to the surface. The constituent particles were also found to be a lot deeper in comparison to its width, which indicates that the surface length of the crack is a poor indication of the crack depth before it has reached the stable crack front shape

Other key features of crack nucleation and short crack growth were also discovered in this study. Around the initiating particle, several cracks were found to have nucleated and had grown together forming a unified crack front. This led to the crack front initially having the shape of the initiating constituent particle before it shortly becomes semielliptical. In addition to the cracks that nucleate at the constituent, additional cracks have been observed to grow on the surface away from the initiating particle. When crack front reaches these cracks, there is an apparent jump in the surface crack length and this is usually seen very close to the nucleation site but may occur as far away as $200 \mu \mathrm{m}$ or further depending on the inclusion shape. It should also be noted that the equivalent precrack sizes (EPS), which are the back-calculated sizes of the nucleation sites using fatigue crack growth models, were found to be similar to constituent particle sizes but not a perfect match. Therefore, it would be untrue to state that EPS is equal to the constituent particle size. 


\subsection{Characterization of Crack Nucleation Sites}

There have been many studies carried out on crack nucleation sites. The bulk of the studies have classified the nucleation sites as one of three different types; equivalent initial flaw size (EIFS), equivalent pre-crack size (EPS), and initial discontinuity state (IDS) [9].

\subsubsection{Equivalent Initial Flaw Size (EIFS)}

At first, the EIFS was used to help assess the effect of external, non-material related flaws such as machining marks and represent them as theoretical cracks that are assumed to exist within the material even before fatigue testing had begun [9]. Since then, with the increased use of the DTA, the EIFS plays a vital role in all life prediction models and has been expanded to cover material flaws and discontinuities as theoretical cracks that are present even in new materials and components [24]. It is very important to note, however, that the EIFS value is imaginary and very model dependent. Currently, the EIFS value (or crack size calculated from a model) does not represent any physical characteristic in the material's microstructure and is only a number, which has been calculated through models and numerical methods (i.e. FASTRAN, NASTRAN and AFGROW). As such, the EIFS value can be easily confused with real, in-service crack sizes. The model may not even indicate the actual crack sizes during any part of the 
fatigue life except at failure and at any point where the model intercepts with actual fractographic data.

Typically, the EIFS was estimated using a three parameter Weibull Distribution fitted to the time to crack initiation (TTCI), which is the time it takes for the crack to reach a set size (i.e. $0.75 \mathrm{~mm}$.), and back-projected using a simple crack growth model [9]. Therefore, the EIFS would follow a Weibull distribution as well.

Of all the crack growth models used to back-calculate the EIFS, the simplest crack growth model took the form:

$$
\frac{D a(t)}{D t}=Q[a(t)]^{\beta}
$$

where $\mathrm{a}(\mathrm{t})=$ crack depth at time $\mathrm{t}$, and

$\mathrm{Q}$ and $\mathrm{b}=$ constants which depend on loading parameters, structural and material properties, etc.

With the introduction of more advanced and accurate crack growth models and also because of the difficulty in obtaining fractographic growth data, a modification was made to the EIFS calculating procedure. This new procedure is an iterative process in which an initial crack size is selected and the crack is grown [9]. The experimental fatigue life is then compared with the fatigue performance predicted by the crack growth model and if they do not match then a new initial crack size is selected. This process continues until the experimental fatigue life is the same as the predicted life from the crack growth 
model. The initial crack size that was used when the two lives matched is assumed to be the EIFS. The accuracy of this methodology is further improved with the addition of some fractographic data when the crack front has reached certain key points. The data at these points are inserted into the model so that we know the predicted life should be fairly accurate in comparison to the actual life. It should be noted, however, that when obtaining the fractographic results, the crack depth is measured from the surface and not from the flaw/crack interface.

The problem with extrapolating back to the initial crack lengths that rely on macro-crack data and models is that the EIFS is usually well within the short crack regime [9]. So the assumptions and data used for the back-calculation are not entirely valid and may not be accurate. As a result, the EIFS values and distribution are only applicable to the particular conditions in which they were derived and cannot be applied in other circumstances.

\subsubsection{Equivalent Pre-crack Size (EPS)}

The crack growth modeling assumptions used in conventional EIFS calculation methods and the non-representative crack growth data used in the models (long crack growth data is used, even in the short crack regime), have led to variations in this standard approach. A modified approach has been developed that minimizes the variability in the EIFS approach [9]. This approach is called the equivalent pre-crack size (EPS) and uses the growth rate immediately around the flaw to characterize the flaw. In order to use this 
method, quantitative fractography must be acquired and used in the region adjacent to the flaw so that accurate data is used rather than model predictions. This will help towards greater accuracy and less variation because it will eliminate a lot of the sensitivity towards the model and data selection used. The EPS approach has even been effectively used when the loading history is complex or incomplete or when the crack configuration is complex.

The term "pre-crack" implies that it is not an actual crack, which eliminates some of the confusion caused by EIFS [9]. Pre-crack is a more appropriate expression than flaw because the defect has been shown to have a crack-like form. The estimation of the equivalent pre-crack size was based on a simple crack growth model instead of a complex cycle-by-cycle loading and backtracking from the fatigue failure life.

This simple crack growth model is based on two basic features found in real aircraft loading sequences. Firstly, the dominant cracks nucleate very early in its fatigue life; it is even possible for the crack to have nucleated within the first loading block. The second feature is that the crack often grows at a constant exponential rate for the majority of the fatigue life. The simple fatigue crack growth model is based on the following relationship [9]:

$$
a=a_{0} e^{B s}
$$

where $\mathrm{a}=$ crack depth, $\mathrm{a}_{0}=\mathrm{EPS}$, 
$\mathrm{S}=$ number of applied loading cycles, blocks, etc, and

$B=$ slope of the line fitted to the log a vs. fatigue loading history.

Deviations have been observed in this model but only as a result of changes in the crack growth mode. This simple crack growth model is based on assumptions that allow it to represent the crack down to a zero crack size. As a result, crack size measurements and quantitative fractography around the initiating flaw must be accompanied by an assumption of the initiating flaw size. Failure to obtain the data required may result in distortion of the crack growth plot.

The initiating flaw size is estimated by ignoring the drop off on the log a vs. fatigue load history plot that is common in the short crack regime and fitting a best-fit curve to the remaining portion of the plot. Following the best-fit curve to the projected crack size prior to loading will give the zero life crack size, which can then be inserted into the crack size data as the first approximation of the EPS. More accurate estimations of the EPS can be obtained through further iterations but, in most cases, it is not usually necessary.

\subsubsection{Initial Discontinuity State (IDS)}

The main difference between the previous two nucleation terms and the initial discontinuity state (IDS) is that the previous two considered a crack that would form on the first cycle of fatigue, which would then be considered as the initial flaw size. The 
IDS is a flaw that is to be assumed to be present in the new material, even before any fatigue loading has been applied.

Previously, Merati et al. [3][4][5] conducted a study on the fatigue related features of the IDS in 2024-T3, 7050-T6, and 7075-T6. The goal was to characterize and quantify the intrinsic discontinuity state within the material as the initial condition for fatigue crack growth to provide a more accurate holistic fatigue life assessment methodology. They conducted fatigue tests and failure analyses of both new and used aluminum components as well as alloys with surface treatments (clad and anodized layers) and also at high and low humidity.

It was found that the sole microstructural discontinuities in the new, bare 2024-T3 aluminum alloys were large and angular constituent particles. Subsequent energy dispersive $\mathrm{x}$-ray (EDX) analysis results led the authors to believe that these particles were iron-bearing particles that were most likely the $\beta$ phase $\left(\mathrm{Al}_{7} \mathrm{Cu}_{2} \mathrm{Fe}\right)$.

It was also found that for the used 2024-T3, which had surface treatments performed such as a clad layer and/or an anodized layer, the IDS was no longer related to constituent particles. It was found that the sole nucleation site during fatigue testing was the clad or anodized surface layers.

Because the constituent particles were found to be the sole microstructural fatigue crack nucleation controlling discontinuity in the new, bare 2024-T3, Merati et al. [3][4][5] 
performed a thorough metallographic examination of material with an emphasis on the constituent particles. Two main conclusions were derived; the larger, angular particles, whose sizes were in the same range as those found at the nucleation site on the fracture surfaces, tended to concentrate in the mid-thickness region in the transverse (LS) and short transverse (ST) planes and that the distribution of the particle sizes followed a cumulative Weibull distribution, where the larger particles, especially those in the range of the size of the ones found at the nucleation sites, made up only a very small fraction of the total particle population.

The authors concluded that the larger, angular particles were usually only found in the mid-thickness region because during the rolling process, the larger constituent particles closer to the surfaces were crushed by the different deformation stress states at the surface and the mid-thickness. The image analysis software would then count these particle clusters as many small particles and not as one big particle. As such, the larger particles were only found in the mid-thickness. It should be noted, however, that a through-thickness metallographic examination was performed as well as an examination through the width of the plate. Both examinations found that there were no significant trends or tendencies through thickness or width.

The constituent particles in each of the three planes (LT, LS, and ST) were examined and in each case, the particle size distribution was found to follow a cumulative Weibull distribution. After measuring the size of the particles found at the nucleation sites, the sizes were superimposed onto these distributions and it was found that the particles that 
were responsible for fatigue crack nucleation of the crack that eventually led to failure was found on the extreme end of the distribution where much less than $1 \%$ of the total particle population was found.

An interesting finding in this study was that there was no correlation found between the IDS size and the fatigue performance of the specimen. In other words, the expectation that a larger IDS size would equate to a shorter fatigue life was not found. The fatigue life was found to be independent of the IDS size. This significant finding further emphasizes the importance of the short crack regime in a complete fatigue life prediction methodology.

\subsection{Crack Front Findings}

Li [11] found that the small crack profile was semi-elliptical with the relationship between the crack depth and surface crack length to be as follows:

$$
\begin{aligned}
& c=0.384(a-0.7) \quad(\text { for } \mathrm{a}>1 \mathrm{~mm}) \\
& c=0.2 \times a(\text { for } \mathrm{a}<1 \mathrm{~mm})
\end{aligned}
$$

where $\mathrm{a}=$ surface crack length, and

$\mathrm{c}=$ crack depth.

This differs from the findings of Grinberg, et al. [25], who reported that short cracks are elliptical and the relation between the crack depth extending beyond the grain boundaries 
and the crack length equals 0.32 and remains constant through the entire short crack regime. They also found that this relationship was independent of stress amplitude and environment.

In addition, other researchers have made similar observations in their work involving short fatigue cracks. Kaynak and Ankara [26] found that the relationship between the crack depth and the surface crack length was, in fact, related to the thickness of the specimen as

$$
\frac{c}{a}=0.9-0.25\left(\frac{a}{t}\right)^{2}
$$

This relationship is only valid for $\mathrm{a} / \mathrm{t}$ ratios $>0.05$ and they did not have any information in regards to cracks when $a / t<0.04$. Carvalho and de Freitas [27] conducted experiments on the aluminum alloy 2090-T8E41 and found a slightly different variation of the same relationship. They found that, in their case, the correlation was

$$
\frac{c}{a}=1.18+0.51\left(\frac{a}{t}\right)^{2}
$$

Swain, et al. [8], although they only had a limited amount of experimental data came to the conclusion that for AISI 4340 steel, single edge notch tensile (SENT) specimens, the relationship was found to be 


$$
\frac{c}{a}=1.0-0.25\left(\frac{a}{t}\right)
$$

\subsection{Murakami's Methodology}

Murakami, et al. [28], in their attempt to model the fatigue performance, proposed that the best parameter used to describe different geometrical shapes is the square root of the defect area projected onto the plane normal to the applied load. However, it should be noted that they did not take into account crack closure effects or non-propagating cracks in their consideration of fatigue cracks.

One of the important features of their model is that, regardless of the material, they used the fact that for $\sqrt{\text { area }_{\text {defer }}} \leq 1000 \mu \mathrm{m}$, the relationship between threshold stress intensity factor and the defect area was $\Delta K_{t h} \propto\left(\sqrt{\operatorname{area}_{d d^{\prime} f e t}}\right)^{1 / 3}$. The aforementioned equation indicates that fatigue limit is dependent on defect or inclusion size by $\sigma_{1} \propto \frac{1}{\left(\sqrt{\text { area }}{ }_{d e f e c t}\right)^{1 / 6}}$ and even though this shows that as the area $\rightarrow 0, \sigma_{1} \rightarrow \propto$ because the lower limit of area is related to the maximum size of non-propagating crack, this equation is still valid.

Experiments have shown that fatigue cracks do, in fact, nucleate below the predicted fatigue threshold, $\Delta \mathrm{K}_{\mathrm{th}}$. However, because defects and constituent particles are regarded as short cracks, the fatigue limit should be used for crack propagation emanating from 
constituent particle clusters or defects and not as a condition for crack initiation/nucleation. Nucleation of short fatigue cracks may occur on the surface of a specimen or within the specimen, wherever a defect or constituent particle may be found.

In the case of surface cracks, the $\sqrt{a r e a_{d e f e c t}}$ may be regarded as the initial surface crack length.

\subsection{Transition from Short to Long Cracks}

Lankford [29] concluded that the growth rates of short and long cracks merge when the plastic zone size is equal to a relevant microstructural dimension.

In terms of investigating and analyzing the transition between short and long cracks, Navarro and de los Rios [30] put forward an expression for this transition:

$$
\Delta K_{t}=\left(\frac{4 \tau_{y}}{\pi \tau_{f}}\right) \Delta K_{t h}
$$

where $\tau_{f}=$ shear stress fatigue limit

Hussain and de los Rios [31] later put forward an expression to determine $\Delta \mathrm{K}_{1}$ at the interface between short and long cracks. This combined with Hyspecky and Strnadel's [32] proposed statistical criterion for the transition between short and long cracks, which can be used to determine the number of cycles for a long crack to form confirms that crack length follows a two parameter Weibull distribution in the form of: 


$$
\rho_{\varepsilon / 2}[a, \gamma(N), \beta(N)]=\beta(N) a^{\beta(N)-1}[\gamma(N)]^{-\beta(N)} \exp \left(-\left(\frac{a}{\gamma(N)}\right)^{\beta(N)}\right)
$$

where $\rho_{\varepsilon / 2}=$ probability density,

$\gamma(\mathrm{N})=$ size parameter, and

$\beta(N)=$ shape parameter.

In the short crack regime, increasing the number of cycles will increase the micro-crack length, $a_{m}$, which is the value where the probability density $\left(\rho_{\varepsilon / 2}\right)$ reaches its maximum and is defined as:

$$
a_{m}(N)=\left(\frac{\beta(N)-1}{\beta(N)[\gamma(N)]^{-\beta(N)}}\right)^{1 / \beta(N)}
$$

It has been suggested that the transition between short and long cracks occur when the micro-crack length, $a_{m}$, remains constant no matter how many further cycles will be performed. Therefore, at this transition point the derivative of the micro-crack length with respect to the number of cycles will be equal to zero as shown:

$$
\frac{d a_{m}(N)}{d N}=0
$$


Inserting equation 10 into equation 11 gives the following mathematical criteria for the transition from short to long cracks:

$$
\frac{1}{\gamma(N)} \frac{d \gamma(N)}{d N}=\frac{1}{\beta^{2}(N)}\left(\ln 3 \beta(N)-14-\ln \beta(N)-\frac{1}{\beta(N)}\right) \frac{d \beta(N)}{d N}
$$

After inserting the dependence of parameters $\gamma(\mathrm{N})$ and $\beta(\mathrm{N})$, a numerical solution will show the number of cycles, $N_{c}$, where the short cracks form into long cracks. This transition from short (stage II) to long crack is graphically shown in Figure 1.

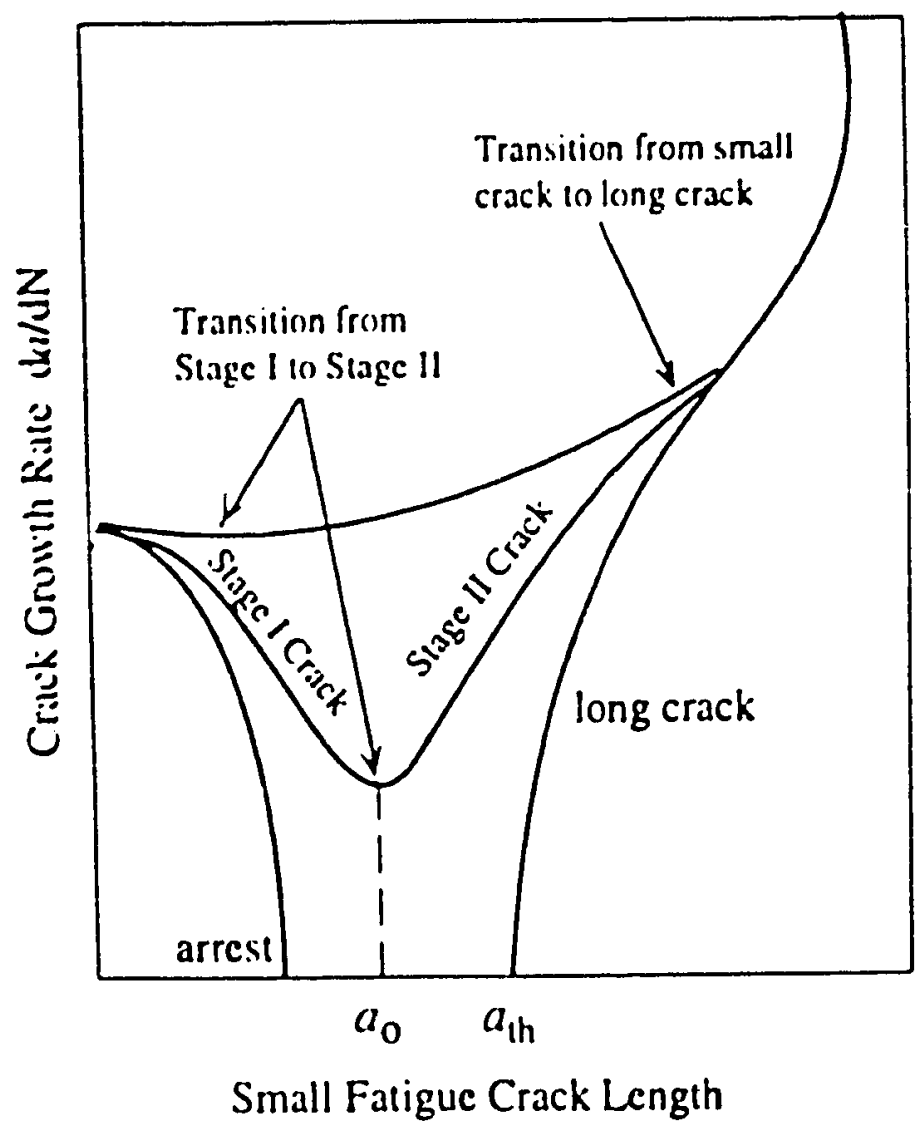

Figure 1. da/dN vs. a curve showing the transition between short and long cracks [11] 


\subsection{Energy Dispersive X-ray (EDX) Analysis}

As mentioned earlier, constituent particles are present in almost all of the fatigue crack nucleation sites. In order to determine which types of constituents are responsible for crack nucleation, a chemical analysis must be performed using the energy dispersive $\mathrm{x}$ ray (EDX) analysis apparatus on the scanning electron microscope (SEM).

First introduced in 1968, this method uses a Si Li-drifted detector to collect the x-ray signals generated by the electron beam, which first must pass through a beryllium window, as shown in Figure 2, where it can then be analyzed by the EDX system. The beryllium window isolates the chamber environment from the $\mathrm{Si}-\mathrm{Li}$ detector and protects it from visible radiation because the detector is very light sensitive.

Silicon is chosen as a suitable radiation detector because it acts like an intrinsic semiconductor, where there are no excess electrons in the outer shell (given that it shares its outermost electrons with its four nearest neighbours) to transport charge, nor are there any vacancies in the outer most shell. Therefore, it will not conduct current in an applied electrical field unless energy is absorbed, consequently freeing up electrons and leaving vacancies behind. However, in practice, it is very difficult to obtain silicon pure enough to achieve this intrinsic condition. Therefore, it is doped with lithium and the lithium is allowed to diffuse into the crystal. The junction between the $\mathrm{Si}-\mathrm{Li}$ will exhibit the necessary intrinsic behaviour. It should also be noted that the doping operation is done at 
temperatures above ambient room temperature and, even at room temperature, lithium remains mobile in the presence of an applied field. Therefore, the $\mathrm{Si}-\mathrm{Li}$ detector must operate at very low temperatures, near the nitrogen liquidus temperature.

The $\mathrm{Si}-\mathrm{Li}$ crystal and the "cold finger", which is the mount that is connected to the liquid nitrogen coolant system and holds the detector crystal, are separated from the rest of the detector housing to provide electrical isolation and to prevent condensation on the cold finger. It is also imperative that the detector be able to mechanically move independently of the specimen without affecting the vacuum inside the electron microscope. This will allow the modification of the count rate by varying the distance between the sample and the detector and hence, the solid angle of the detector acceptance.

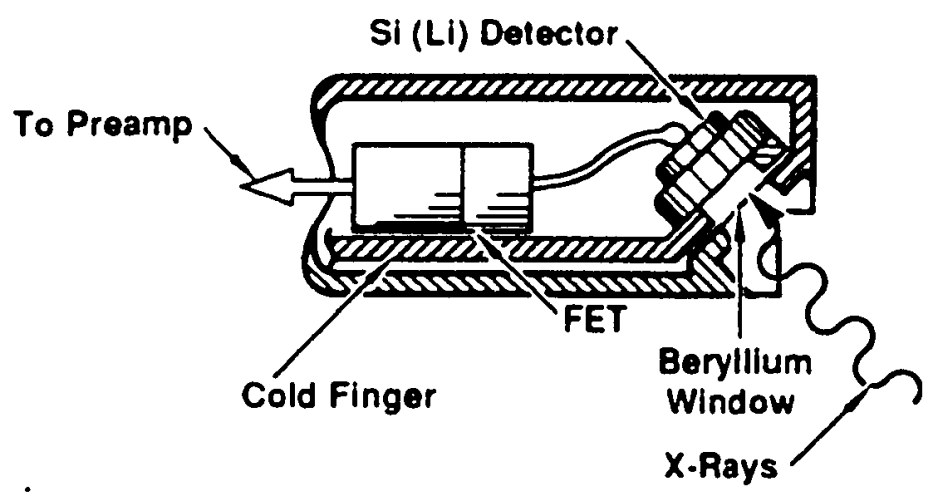

Figure 2. Cross-section of the $\mathrm{Si}-\mathrm{Li}$ detector [33]

The absorption of an $\mathrm{x}$-ray photon will lead to the release of a photoelectron and these photoelectrons lose most of their energy to the formation of electron - pairs that are, in turn, swept away by the applied bias to create a charge pulse, shown in Figure 3. Using a 
preamplifier (charge - to - voltage converter), the charged pulse is then converted to an electric potential voltage. After the voltage is further amplified, it is passed through a computer $x$-ray analyzer as shown in Figure 4.

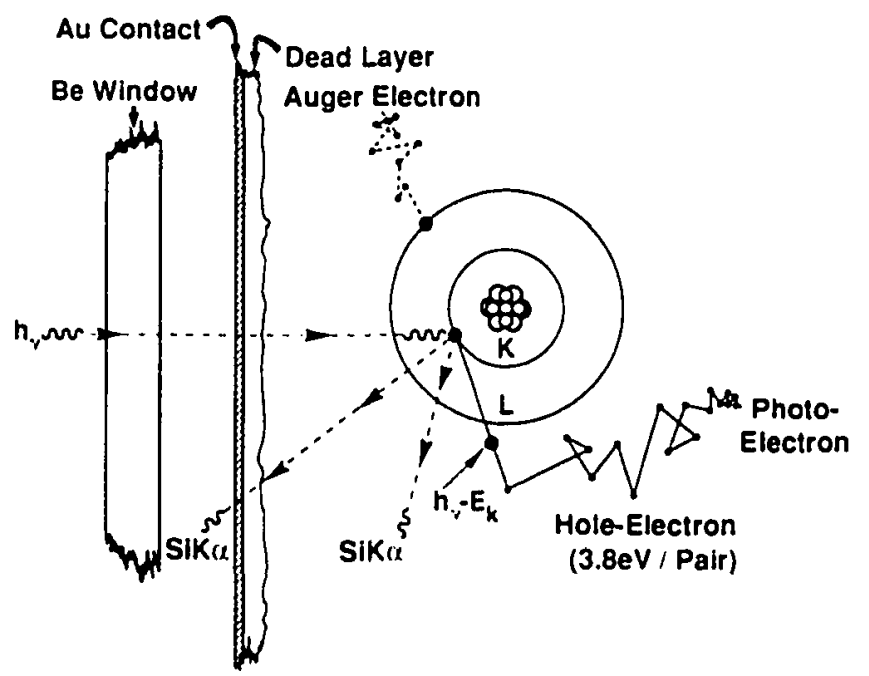

Figure 3. The $x$-ray detection and photoelectron creation process [33]

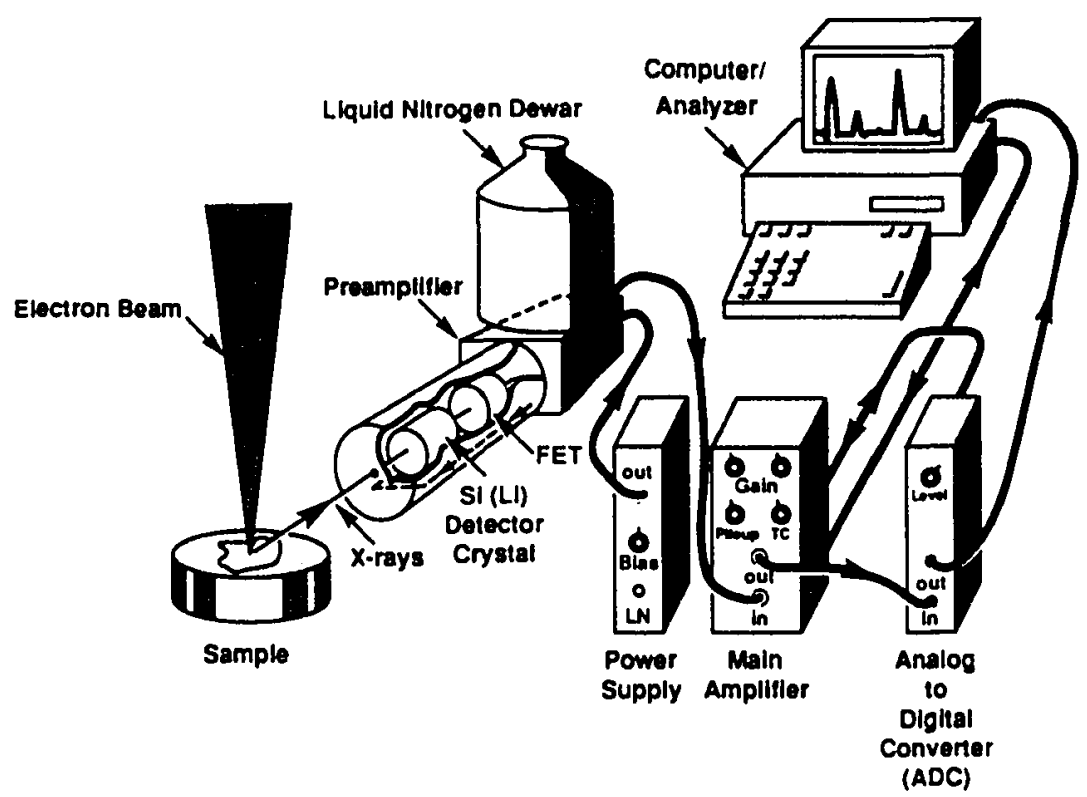

Figure 4. Schematic of the EDS system [33] 
Each element has unique energy characteristics (i.e. $k_{\alpha}$ radiation energy) and each detected potential voltage is proportional to the incoming $x$-ray photon energy. Once the analysis is done, the EDX system catalogues the data according to Figure 5. It should be noted that the typical resolution of the EDX system is $50 \mathrm{eV}$, which means that it cannot accurately detect or separate anything finer than that. These results in peak overlap and peak pile-up of elemental energy peaks that are close together.

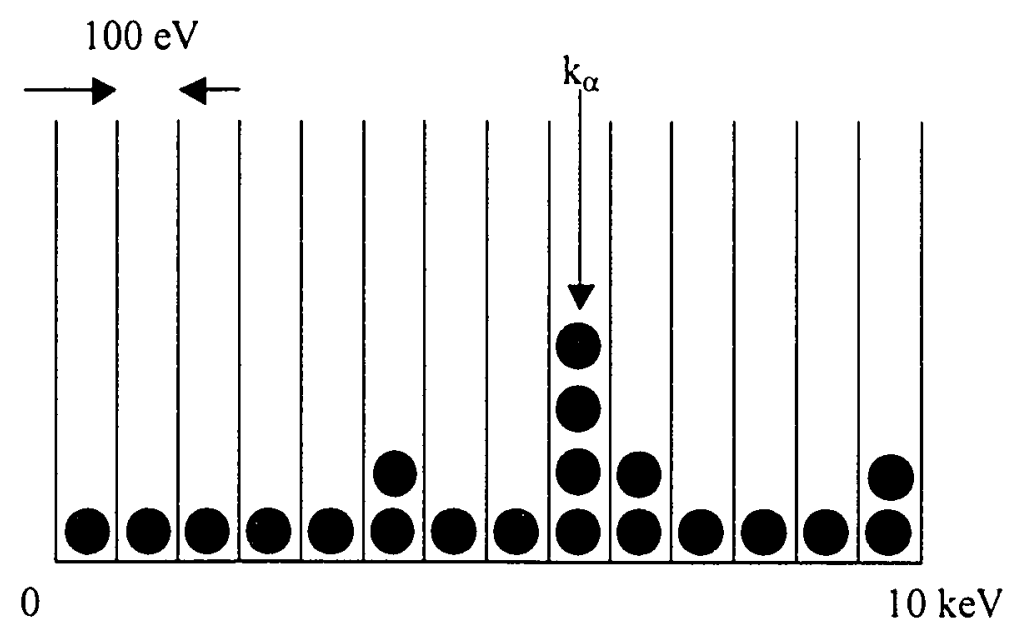

Figure 5. EDX sorting of characteristic $x$-ray photon data. Each column would represent a certain element with those certain $\mathrm{x}$-ray energy characteristics. In this example, the EDX resolution is $100 \mathrm{eV}$.

Merati et al. [3][4][5] performed an EDX analysis on the 2024-T3 that they fatigue tested. They found that there were basically two distinguishable groups of particles; small, round particles and large, angular particles. The large, angular particles were found to contain a significant amount of iron and silicon while the smaller and rounder particles, on the other hand, did not contain any iron or silicon. They also found that these larger angular particles contained noticeable amounts of manganese and copper. Afterwards, an identical analysis was conducted on the particles that they found at the crack nucleation 
sites and the results from these particles were identical to the large, angular particles. Based on this EDX analysis, they concluded that only the iron and silicon bearing particles contributed to fatigue crack nucleation. They were also able to conclude, based on the ASM metals handbook, that the larger particles were possibly $\mathrm{Al}_{7} \mathrm{Cu}_{2} \mathrm{Fe}$ ( $\beta$-phase), $\mathrm{MgSi}_{2}$, and $\mathrm{Al}_{12} \mathrm{Si}(\mathrm{Fe}, \mathrm{Mn})_{3}$ and the smaller particles were mainly $\mathrm{CuMgAl}_{2}$ (S-phase).

\subsection{Electrical Potential Drop (EPD) Technique}

One of the greatest difficulties in studying the short crack characteristics of aluminum alloys was being able to accurately and consistently detect these cracks during fatigue testing. The difficulty in detecting and monitoring short cracks has resulted in the short crack regime of high strength aluminum alloys not being as established as the long crack regime and why a lot of the findings are still presently being debated. An important part of this thesis work is establishing a short crack monitoring technique and it seems as though the most promising technique is the electrical potential drop (EPD) method.

EPD has been a popular technique for the measurement of crack growth rates in many metallic samples. It has always been a popular choice because of its sensitivity, flexibility, and also because this technique is conducted, measured, and analyzed through an automated process. The use of electrical potential measurements to determine crack size is based on the principle that the electrical field in a sample with current flowing through it is based on the geometry of the specimen and as such, will be altered with a presence of a crack. In the case of using DC current (constant current flow), the electrical 
potential across the crack plane will increase when a crack increases in size because of the modification of the electrical field and the associated perturbation of the current flow lines. This change in voltage can be related to the crack size through a secondary crack measurement technique, which in this case, were marker bands.

The EPD method determines the fatigue crack growth rates from near-threshold $\left(\mathrm{K}_{\mathrm{th}}\right)$ to the maximum stress intensity factor $\left(\mathrm{K}_{\max }\right)$. The fatigue threshold is defined as the value of $\Delta \mathrm{K}\left(=\mathrm{K}_{\max }-\mathrm{K}_{\min }\right)$ for which the crack growth rate $(\mathrm{da} / \mathrm{dN})$ approaches zero. Experimentally, it is very difficult to find the $\Delta \mathrm{K}$ value for which $\mathrm{da} / \mathrm{dN}$ equals zero; therefore, the commonly used value for the fatigue threshold is when the $\mathrm{da} / \mathrm{dN}$ is at a rate of $10^{-10} \mathrm{~m} /$ cycle. Typically, the results of the EPD methodology are provided in terms of the stress intensity factor range $(\Delta K)$, as defined by linear elasticity. Any sample design can be used as long as the specimens are of sufficient thickness and size according to the ASTM E647 standard and that deformation will remain primarily elastic during fatigue testing.

It should be noted however, local crack tip effects, such as residual stresses and crack closure, may significantly affect the crack growth rates, especially at low stress intensity factors $(K)$ and low stress ratios $(R)$. The calculation of $\Delta K$ using linear elasticity does not incorporate these effects and hence, is not immediately accounted for in EPD results. The relationship between the loading parameters $\Delta K, R$, and $K_{\max }$ follow the following relationships: 


$$
\begin{gathered}
\Delta K=(1-R) K_{\max } \text { for } R \geq 0 \text {, and } \\
\Delta K=K_{\max } \text { for } R \leq 0
\end{gathered}
$$

\subsubsection{Use of Electrical Potential Difference (EPD) for Measuring Crack Size}

The use of electrical potential difference (EPD) for measuring crack size is applicable in any conductive material in any environment and is only be possible with the assistance of an alternative technique such as optical microscopy or marker bands. The use of an alternative technique is important because it will provide accurate calibration points of the crack size on certain EPD values so that a relationship can be obtained for EPD vs. fatigue crack size. As evident, the use of two techniques to obtain an accurate relationship is very important where a parameter (i.e. stress intensity factor), which is derived from the crack size, is being controlled during the fatigue testing.

During an axial fatigue test, the voltage measurements should be taken at or near the peak of the axial force because of potential shorting of the fracture surface. In an oxidizing environment where an oxide layer would form on a "fresh" surface, this oxide layer would act as an insulator and prevent any shorting between the two halves of the specimen. However, in an environment where the oxide layer has not formed yet or where high fracture surface closure forces are present, the two halves may not be insulated from each other and shorting may occur which would give inaccurate voltage readings. 
Another consideration in EPD testing is that elastic and plastic deformation may affect the material's resistivity. However, correction for shorting errors in fatigue testing will generally account for the deformation effects on the electrical properties of the material.

It is also important to note that changes in either the specimen or instrumentation may have significant effects on the voltage readings. For example, a temperature change in the specimen may alter the material's resistivity and henceforth, the measured electrical potential drop across the specimen. The simplest and most effective way to take into account any changes in the specimen or instrumentation is to take an additional voltage reading on a reference specimen or at a reference location on the same specimen. Taking an additional voltage reading would help correct any changes in the material or instrumentation because the reference specimen or location would also be exposed to any material or instrumentation variation and by normalizing the specimen signal and the reference signal, you can significantly increase crack resolution and account for any material and instrumentation variations.

In EPD testing, the general output voltage is very minimal, in the range of 0.1 to $50 \mathrm{mV}$ for a current output of 5 to $50 \mathrm{~A}$. Of course, the measured voltage drop has a lot to do with the resistance of the material being tested. The higher the conductivity, the lower the voltage and the greater the current required to obtain the same voltage output. In a very conductive specimen such as an aluminum alloy, the applied current must be very high in order to have a measurable voltage output. However, with a higher current output, the possibility of heating up the fatigue sample would greatly increase and with 
such a temperature increase, the material's resistivity would also be affected. This is of particular concern for aluminum alloys because of their low aging and melting temperatures. Therefore, in order to use a high current output and still have accurate results without affect material properties, the current and voltage readings must be pulsed rather than continuous. As a result, tradeoffs have to be made between crack sensitivity and response time of the test data, especially when dealing with conductive materials like 2024-T3.

The EPD method is also susceptible to thermoelectric effects which produce a voltage output in addition to those produced by the specimen's electric field. This additional voltage may account for a significant portion of the total measured voltage and therefore, should be taken into account. There are two methods of correction used to account for the thermoelectric effects. The first method is to subtract the measured voltage when the current is not being applied to the measured voltage when the current is flowing through the system. The second method is to reverse the current flow direction and take voltage measurements when the current is flowing in both directions. The corrected voltage measurements will then be one-half of the difference of the measured voltage taken from both current directions.

The placement of the voltage wire must also be very carefully considered. Typically, the trouble with choosing a voltage wire location is being able to find a suitable compromise between crack size sensitivity and location sensitivity (errors from small variations in voltage wire location). Choosing a voltage wire location near the crack tip or notch tip 
will yield better crack sensitivity but because of the non-uniformity of the electric field near the crack tip area, small variations in the voltage lead wire locations may significantly alter the voltage readings. The general rule of thumb is that the greater the crack sensitivity, the greater the location sensitivity. It should also be noted that the voltage wires should be positioned diagonally across the notch or crack in order to obtain average measurements from non-uniform crack fronts.

The selection of current input wires should be made on the basis of that wire's current carrying capability as well as the ease of connectivity/attachment. The connectivity basis is of secondary importance because even though the wire may not be welded onto the test sample, it may be mechanically fastened. The most significant criteria is the wire's current carrying capability. As such, the gage of the wire should be chosen so that it may carry the current being supplied without significant heating. In other words, the wire should be made of a material with low resistance.

The selection of the voltage lead wires should be such that the wire is as fine as possible in order to reduce the amount of stress on the wire and prevent any detachment. Also, the wire should be as thin as possible to allow for more precise positioning of the voltage lead wire locations. While the current lead wires can be mechanically fastened to the fatigue sample, the voltage lead wires should be welded onto the sample to ensure a reliable and consistent joint. 
A further consideration with the voltage wires is induced electromagnetic fluctuations (EMF). One of the methods to avoid this is to try and keep the voltage wires as short as possible and to have them twisted in order to reduce stray voltages as a result of fluctuations of magnetic fields. Holding the wires rigid would also help because moving a wire through a static magnetic field that may exist near the test frame will induce stray voltages. Lastly, it would be beneficial to make sure that the voltage wires stay as far away as possible from any equipment that produce strong magnetic fields.

As discussed earlier, thermal effects on the fatigue specimen may play a significant role in the results of the EPD method. The thermal effects of the equipment may also play a role in the accuracy of the data obtained. To reduce these effects, the minimum number of connections should be used and all of the connections and equipment should be maintained at a constant temperature.

Another careful consideration to provide accurate EPD results is to make sure the fatigue specimen is properly insulated from the test frame. It is vital that no portion of the applied current is connected in a parallel circuit through the test machine. In general, the resistance of the test frame is significantly greater than the fatigue specimen; however, there are some situations in which an alternative current path may exist through the test frame. Therefore, the fatigue specimen should be isolated from the test frame to ensure that the current flowing through the reference specimen is exactly the same amount of current that is flowing through the fatigue specimen loaded in the test frame. This would 
also ensure that the measured current output from the power source is the same amount of current flowing into the specimen.

The power source used to provide the current will need a stabilization period. Before any of the EPD tests start, the power supply should be turned on and be given sufficient time to stabilize. The duration of the stabilization time is dependent on the power supply being used and should be obtained with experimentation. This is important in situations where the power supply is first turned on and when pulse readings are being taken. Because aluminum is highly conductive, the crack resolution would be lower. In order to compensate for the decreased crack resolution, a higher current output would have to be used. The selection of a proper current output is difficult because choosing too low a current would result in decreased crack resolution whereas choosing too high a current will cause significant heating and arcing of the fatigue sample. Therefore, to use a higher current output to obtain to required crack sensitivity without heating up the specimen, the current would only be switched on just before taking a voltage reading and would be switched off right after the reading has been taken. This is what is referred to as pulse readings because the readings are taken at set time intervals rather than a continuous reading.

\subsubsection{Significance and Use of EPD Results}

Analysis of EPD-produced crack growth data typically presents the crack growth rate data as a function of the crack-tip stress intensity factor (da/dN vs. $\Delta \mathrm{K})$. The significance 
of this data is that it gives a measure of the materials resistance to stable crack growth under cyclic loading. Another advantage of expressing the crack growth rate as da/dN vs. $\Delta \mathrm{K}$ is that this result will be independent of planar geometry. This will allow for comparison of data that have been obtained with different specimen configurations and/or loading conditions. This representation also implies a very significant assumption; that cracks, even though they may vary in size, will experience the same crack extension per cycle when subjected to the same nominal $\Delta \mathrm{K}$.

Even though expressing the crack growth rate data as da/dN vs. $\Delta \mathrm{K}$ causes the data to be independent of planar geometry, this may not always be the case. There have been mixed opinions of the effect of thickness on this crack growth data. The fatigue crack growth rates over the whole range of $\Delta \mathrm{K}$ have been reported to increase, decrease or stay the same with increasing thickness [34][35]. Other factors that may affect the crack growth rate are residual stresses and crack closure effects. Residual stresses may play a significant role on specimens which have been taken from material which have not had proper stress relief. Residual stresses in conjunction with the applied stresses during the fatigue testing will cause the crack-tip stress-intensity factor $(\mathrm{K})$ to be different from the computed value, which is based solely on the applied load of the fatigue testing. This may lead to compressive forces on the specimen even though the fatigue test was planned to be entirely tensile and vice versa. A good indication of the presence of residual stresses is irregular crack growth, particularly out-of-plane crack growth or excessive crack front curvature. Crack closure can also have a very significant effect on fatigue crack growth rates, particularly near the fatigue threshold at low stress ratios $(\mathrm{R})$. As a 
result, it can be seen that the loading history and the conditions in the wake of the crack can play a significant role on the current propagation rates of the fatigue crack. Crack closure affects the crack propagation rates because the effective stress intensity factor $\left(\Delta \mathrm{K}_{\mathrm{effi}}\right)$ at the crack tip differs from the nominally applied stress intensity factor $(\Delta \mathrm{K})$ given the loading cycle. This implies non-unique growth rate dependence in terms of $\Delta \mathrm{K}$ and $\mathrm{R}$. Therefore, a more accurate approximation of the short crack regime can be attained by fatigue testing at a high stress ratio where the effect of crack closure will be minimized.

\subsubsection{Calibration Notes}

The precision of the results obtained from the EPD technique are only as good as the calibration performed on the output voltage to every crack length. An accurate calibration curve is the most important process in the EPD process and hence, most of the emphasis is put on the calibration. According to ASTM standards (E 647-00) [34], the recommended calibration for a CT sample is (for $0.24 \leq \mathrm{a} / \mathrm{W} \leq 0.7$ ):

$$
\frac{a}{w}=-0.5051+0.8857 \frac{V}{V_{r}}-0.1398\left(\frac{V}{V_{r}}\right)^{2}+0.0002398\left(\frac{V}{V_{r}}\right)^{3}
$$

where $V_{r}=$ reference crack voltage corresponding to $\mathrm{a} / \mathrm{W}=0.241$.

While this calibration may account for material and specimen geometry variations, other sources of variations are not taken into account. These variations include electrical noise, 
instrumentation variations, and any thermo-electrical effects. However such variations can be accounted for with the use of an identical reference specimen that is connected along the same current path.

It was shown by Tong [35] that variations in the position of both the current and potential leads does not lead to significant differences in the measured voltage drop across the notch of a CT specimen. The current and potential lead wire locations were varied by increments of a small distance, $\Delta$, and it was shown that the variations in the location of the lead wires had very little effect on the relationship between the potential drop and crack size. There results are in Figure 6 and Figure 7.

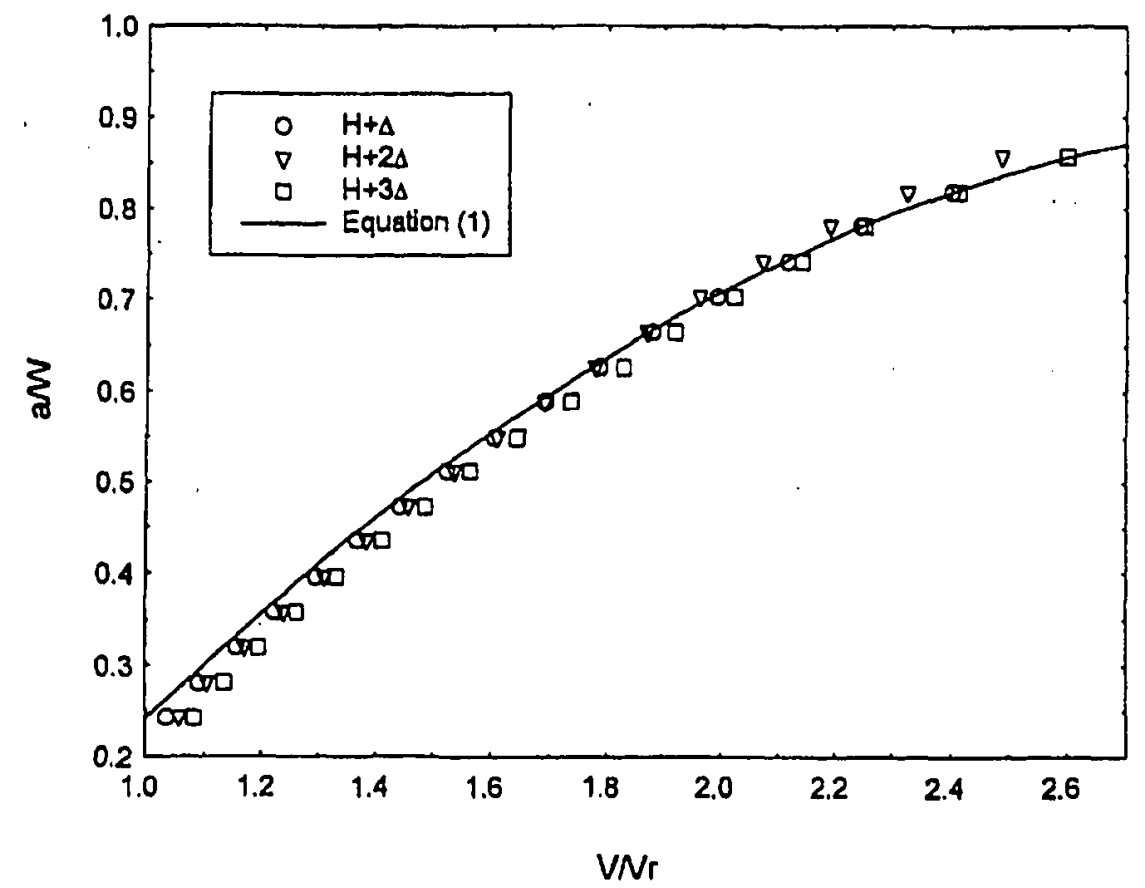

Figure 6. Influence of different potential lead locations on measured potential differences [35] 


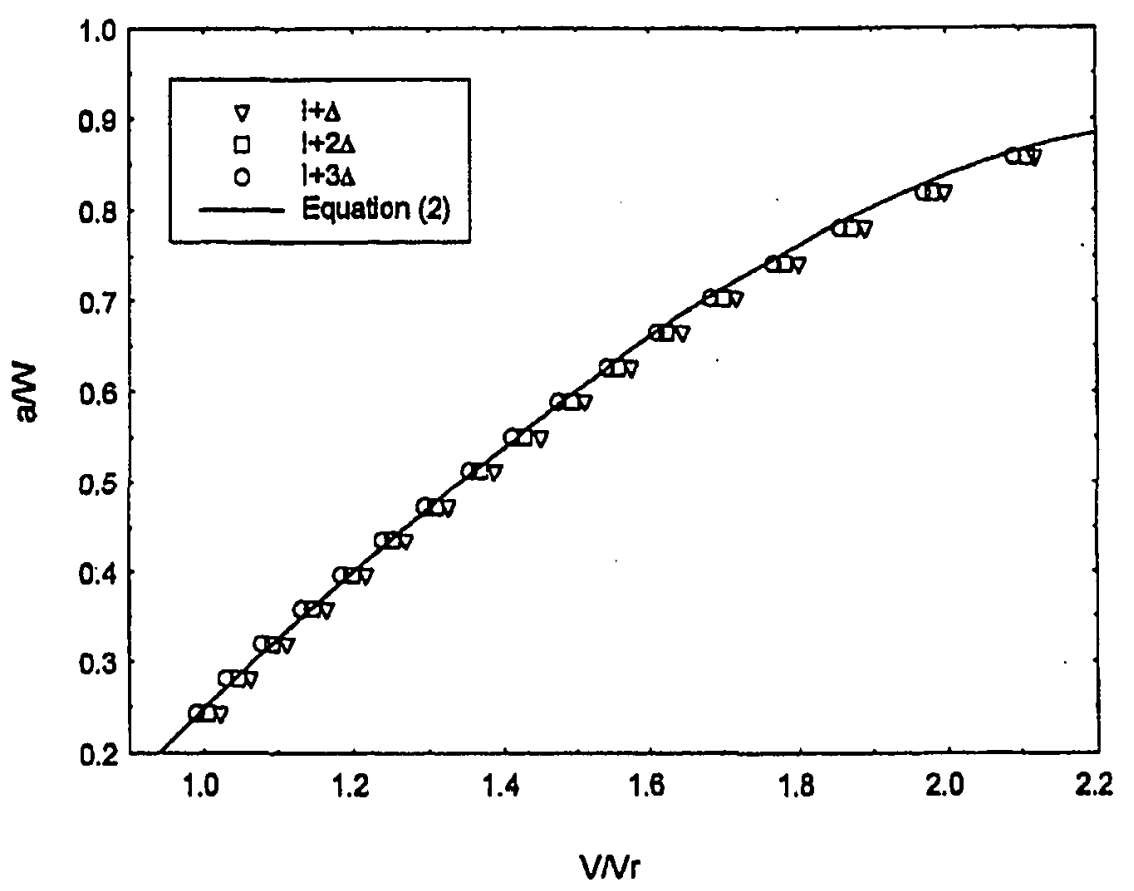

Figure 7. Influence of different current lead locations on measured potential drops [35]

The threshold stress intensity factor for fatigue crack growth is determined by first plotting the linear regression line on the $\log (\mathrm{da} / \mathrm{dN})$ vs. $\log (\Delta \mathrm{K})$. This plot must be made of at least five da/dN and $\Delta \mathrm{K}$ points, spaced accordingly between the growth rates of $10^{-9}$ and $10^{-10} \mathrm{~m} /$ cycle. From this plot, calculate the value of $\Delta \mathrm{K}$ when the growth rate is $10^{-10} \mathrm{~m} / \mathrm{cycle}$ and this value will be identified as the threshold crack intensity factor, $\Delta \mathrm{K}_{\mathrm{th}}$. 


\subsection{EXPERIMENTAL PROCEDURES AND MATERIALS}

This section provides background information on the material tested in this thesis as well as outlining the experimental procedures used during the metallographic examination and fatigue testing.

\subsection{Material}

The $2 \mathrm{xxx}$ series aluminum alloys contain copper as the primary alloying element and often with magnesium as a secondary addition [36]. These alloys require solution heat treatment to obtain optimal properties and in some cases, require precipitation heat treatment (aging) to obtain a further increase in mechanical properties. The addition of copper allows the $2 \times x \times$ series to be heat treatable (solution heat treatment and aging) and depending on the other constituents present, the maximum strengthening is achieved with a copper content between 4-6\%. Magnesium also provides strengthening following heat treatment and quenching. It also increases the strength of the alloy and ductility during natural aging. Natural aging refers to the spontaneous formation of a Guinier-Preston (GP) zone structure during exposure to room temperature. The solute atoms either cluster or segregate to selected atomic lattice planes, depending on the alloy system, to form the GP zone and this zone is more resistant to dislocation movement through the lattice and makes the alloy stronger [36]. 2024 is an alloy that is widely used in the natural aging condition because it becomes stable quickly at room temperature. 
For 2024, other elements present are manganese, chromium, titanium, zinc, iron and silicon [36]. Titanium is added to 2024 because it is used to refine the as-cast grain structure and has little effect on the changes in grain size during recrystallization or mechanical working. Chromium is added to control the grain structure, prevent grain growth and to prevent recrystallization during hot working and heat treatment because it has a slow diffusion rate and forms fine dispersed phases, which inhibit nucleation and grain growth. If chromium is added in excess of $0.35 \%$, it tends to form very coarse constituents with other impurities such as manganese, iron and titanium. Iron is the most common impurity found in aluminum alloys and because the solubility of iron in the solid state is very low $(\sim 0.04 \%)$, most of it appears as a second phase in combination with aluminum and other elements. An example is when the excess iron unites with copper to form the $\mathrm{Cu}_{2} \mathrm{FeAl}_{7}$ constituent, which reduces the amount of copper available for heattreating effects and lowers tensile properties in the heat treated condition. Silicon is added to tie up the iron content by forming the $\alpha \mathrm{FeSi}$ constituent and if there is sufficient silicon to tie up all the iron content, the mechanical properties of the alloy will be unaffected. Also, the presence of silicon and magnesium allows the alloy to be more heat treatable because the silicon and magnesium combine to form the $\mathrm{Mg}_{2} \mathrm{Si}$ precipitate during the aging process. Lastly, manganese is used to increase the strength in either the solid solution or as a finely dispersed precipitate phase during mechanical working by refining the grain size and shape. Manganese also increases the recrystallization temperature and as a dispersed precipitate, it is effective in slowing recovery and preventing grain growth. Manganese is also used to correct the shape of iron-bearing constituents to reduce the embrittling effect and if the iron and manganese content is high 
enough, it will form the $(\mathrm{Fe}, \mathrm{Mn}) \mathrm{Al}_{6}$ constituent phase during casting. Even though the addition of manganese is beneficial, the concentration of it does not exceed $1 \%$ in commercial aluminum alloys because it will significantly decrease ductility [36].

No element is known to have complete $(100 \%)$ solid state solubility in aluminum and when the alloying content exceeds the solid-solubility limit, a second-phase microstructural constituent phase is produced. Zinc has the greatest solubility in aluminum at 66.4 at\% with magnesium with the second highest at over 10 at $\%$. Silicon has a solid-solubility of less than 10 at $\%$ in aluminum and copper has just over 1 at $\%$. All of the remaining alloying elements have solid-solubilities of less than 1 at $\%$. Manganese and chromium in particular have very low solid-solubilities and readily form secondary phase constituents. In cases where manganese, iron and silicon are present, the $\mathrm{Al}_{12}(\mathrm{Fe}, \mathrm{Mn})_{3} \mathrm{Si}$ phase is formed. $\mathrm{Al}_{20} \mathrm{Cu}_{2} \mathrm{Mn}_{3}$ is formed in alloys containing copper and manganese and when both chromium and magnesium are present, $\mathrm{Al}_{12} \mathrm{Mg}{ }_{2} \mathrm{Cr}$ is produced. As a result, since 2024 contains manganese, iron, silicon, copper, chromium and magnesium, the possible secondary phase constituents present are $\mathrm{Al}_{12}(\mathrm{Fe}, \mathrm{Mn})_{3} \mathrm{Si}$, $\mathrm{Al}_{20} \mathrm{Cu}_{2} \mathrm{Mn}_{3}$, and $\mathrm{Al}_{12} \mathrm{Mg}_{2} \mathrm{Cr}[36]$.

The material used in this thesis was taken from new stock of 2024-T3. The alloy had a bare surface and no surface treatments such as cladding or anodizing had been applied. All of the metallography and fatigue samples were taken from this $4.064 \mathrm{~mm}$ thick sheet of 2024-T3. This alloy was chosen because of the extensive fatigue test data available and because it is used in many important structural components of aircraft. Another 
benefit of this alloy is that it was readily available and previous fatigue studies [3][4][5] performed with this same batch of material provided very consistent fatigue test results.

The 2024 has largely superseded 2017 for structural applications and is chosen because of its favorable strength-to-weight ratio and good machinability, workability, and surface finish capabilities. This alloy is typically used in applications such as aircraft fittings, gears and shafts, bolts, and many others.

Presented below are the compositional ranges for alloying elements in the 2024 alloy.

Table 1. Composition of AA2024 [36]

\begin{tabular}{|c|c|c|c|c|c|}
\hline Component & $\mathrm{Wt} \%$ & Component & $\mathrm{Wt} \%$ & Component & $\mathrm{Wt} \%$ \\
\hline $\mathrm{Al}$ & 93.5 & $\mathrm{Fe}$ & $\mathrm{Max} 0.5$ & $\mathrm{Si}$ & $\mathrm{Max} 0.5$ \\
\hline $\mathrm{Cr}$ & $\mathrm{Max} 0.1$ & $\mathrm{Mg}$ & $1.2-1.8$ & $\mathrm{Ti}$ & $\mathrm{Max} 0.15$ \\
\hline $\mathrm{Cu}$ & $3.8-4.9$ & $\mathrm{Mn}$ & $0.3-0.9$ & $\mathrm{Zn}$ & $\operatorname{Max} 0.25$ \\
\hline
\end{tabular}

The $\mathrm{T} 3$ heat treatment designation signifies that the 2024 has undergone a heat treatment in order to further enhance the strength-to-weight ratio. The T3 heat treatment involves solution heat treatment, cold working, and then natural aging. The generic properties of the 2024-T3 alloy are shown in Table 2. 
Table 2. Minimum mechanical properties of AA2024-T3 in the longitudinal (rolling) direction [37]

\begin{tabular}{|c|c|c|}
\hline Mechanical Property & Metric & Imperial \\
\hline Yield Strength & $290 \mathrm{MPa}$ & $42000 \mathrm{psi}$ \\
\hline Tensile Strength & $440 \mathrm{MPa}$ & $64000 \mathrm{psi}$ \\
\hline Elongation at Break & $18 \%$ & $15 \%$ \\
\hline Modulus of Elasticity & $72.4 \mathrm{GPa}$ & $10500 \mathrm{ksi}$ \\
\hline Fatigue Strength* & $138 \mathrm{MPa}$ & $20000 \mathrm{psi}$ \\
\hline
\end{tabular}

* Based on a 500,000,000 cycle fatigue life and stress ratio of -1

\subsection{Procedures}

The procedures for metallography, image analysis, Microsoft Excel analysis, fatigue specimen preparation, and fatigue testing are presented below.

\subsubsection{Metallography and Image Analysis}

Using standard metallography procedures, all of the samples were mounted in Bakelite "pucks". Each sample was polished first with SiC paper, specifically the 240, 320, 400, and 600 grit papers. The samples were then polished with a diamond abrasive solution ( 9 $\mu \mathrm{m}, 6 \mu \mathrm{m}, 3 \mu \mathrm{m}$, and $1 \mu \mathrm{m}$ ) before undergoing the final polishing stage. The last polishing stage was conducted with a colloidal solution containing $\mathrm{SiO}_{2}$, which gives a surface finish of approximately $0.05 \mu \mathrm{m}$ rms (root mean square). 
Due to the resulting anisotropic behaviour after the rolling operations, all three planes longitudinal-transverse (LT), longitudinal-short transverse (LS), and transverse-short transverse (ST) were examined and studied. The three planes are defined by the two directions that make up that plane. For example, the LT plane is defined by the two perpendicular directions longitudinal $(\mathrm{L})$ and transverse $(\mathrm{T})$ to the rolling direction. Two samples of each plane were prepared and one was left in the as-polished condition to be examined under scanning electron microscopy and the other sample, which was examined under optical microscopy, was etched with Keller's reagent. Keller's reagent is the most commonly used etchant for aluminum and aluminum alloys, except those with high silicon content. Keller's reagent is composed of the following chemicals:

Table 3. Composition of Keller's reagent

\begin{tabular}{|c|c|c|c|}
\hline Nitric Acid & $\mathrm{HCl}$ & $\mathrm{HF}$ & Water \\
\hline $2.5 \%$ & $1.5 \%$ & $1 \%$ & $95 \%$ \\
\hline
\end{tabular}

* Percentages are based on volume

Examination with backscattered electrons under the scanning electron microscope provided digital images with the constituent particles clearly visible. The images were examined with an image analysis software called ImagePro and the resulting data was exported into Microsoft (B) Excel for further analysis. The step by step procedure for the Image Pro Analysis software can be found in the Appendix A. A full list of the type of data collected during the image analysis is outlined along with the range of the data in Table 4. Figure 8 shows a typical SEM micrograph analyzed using ImagePro. A total of 100 micrographs (10 rows of 10 pictures) were taken at $500 \mathrm{X}$ magnification for each 
plane for a total analyzed area of $2 \mathrm{~mm}^{2}$. Figure 9 shows how the $100 \mathrm{SEM}$ micrographs were positioned to examine the through-thickness distribution of constituent particles. During the analysis of the constituent particles, a lower limit of $0.50 \mu \mathrm{m}^{2}$ was imposed on the particles examined. This lower limit was necessary to eliminate some possible errors in the analysis because the limitations of the screen's resolution equate to about $0.50 \mu \mathrm{m}^{2}$ and particles smaller than this may not be clearly distinguishable.

Table 4. Ranges for the measurement of different physical properties of the constituent particles

\begin{tabular}{|l|l|}
\hline Angle & $0-180$ \\
\hline Area & $0.50-\max { }^{*}$ \\
\hline Aspect & $0-\max$ \\
\hline Axis (Major) & $0-\max$ \\
\hline Axis (Minor) & $0-\max$ \\
\hline Area Fraction & $0-1$ \\
\hline Box Height & $0-\max$ \\
\hline Box Width & $0-\max$ \\
\hline Box X/Y & $0-\max$ \\
\hline Perimeter & $0-\max$ \\
\hline Radius (Max) & $0-\max$ \\
\hline Radius (Min) & $0-\max$ \\
\hline Roundness & $0-\max$ \\
\hline Size (length) & $0-\max$ \\
\hline Size (width) & $0-\max$ \\
\hline
\end{tabular}

* Max is approximately $10^{8}$ in order to ensure that all upper data was included during the analysis 


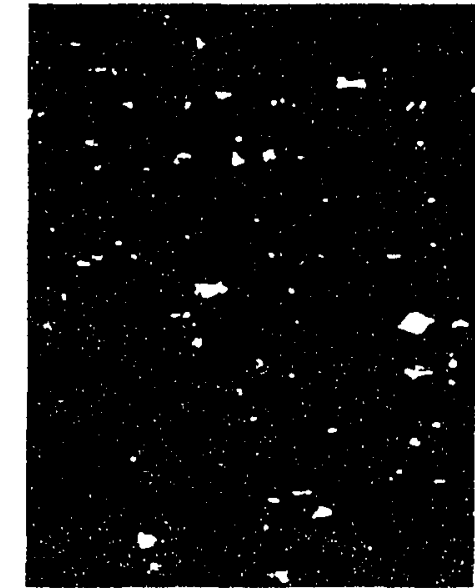

a)
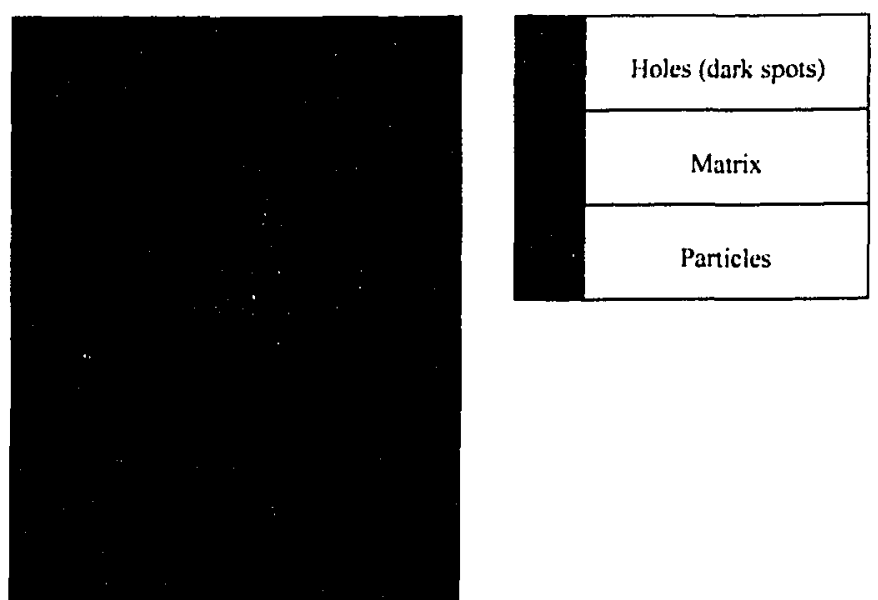

b) After setting ranges for analysis

Figure 8. A typical example of the image analysis performed on the SEM micrographs using the ImagePro Software.

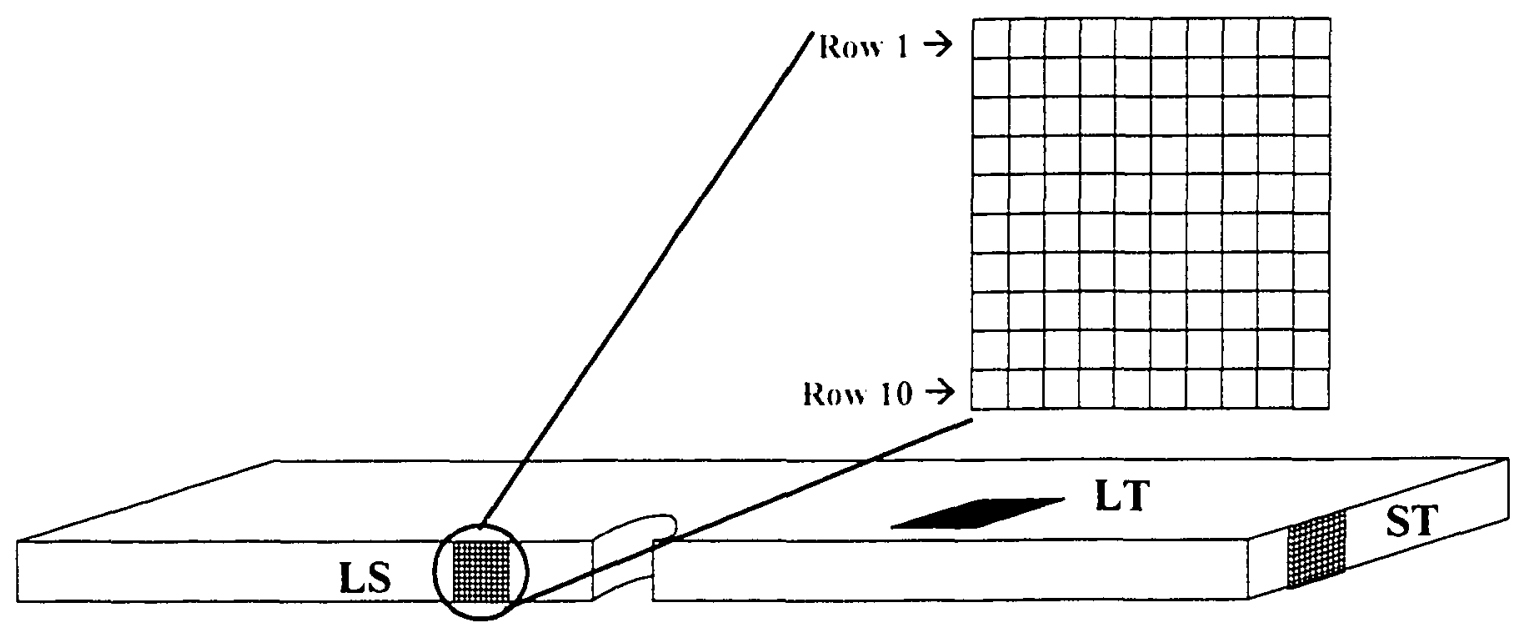

Figure 9. Schematic showing the location of pictures taken during metallography. For the LS and ST planes, the locations were chosen such that a through-thickness microstructural analysis was performed. Ten rows of ten pictures were taken in each plane for a total examination area of over $2 \mathrm{~mm}^{2}$.

\subsubsection{Microsoft Excel Analysis Procedure}

After the image analysis is complete, the data must be exported into Microsoft Excel for further analysis. The step-by-step procedure for this analysis is in Appendix B. 


\subsubsection{Fatigue Testing Procedure}

A single-edged notched specimen was chosen in order to facilitate the evaluation of different detection techniques; surface replication, marker bands, and direct current potential drop (DCPD). A schematic of the fatigue sample design is shown in Figure 10.
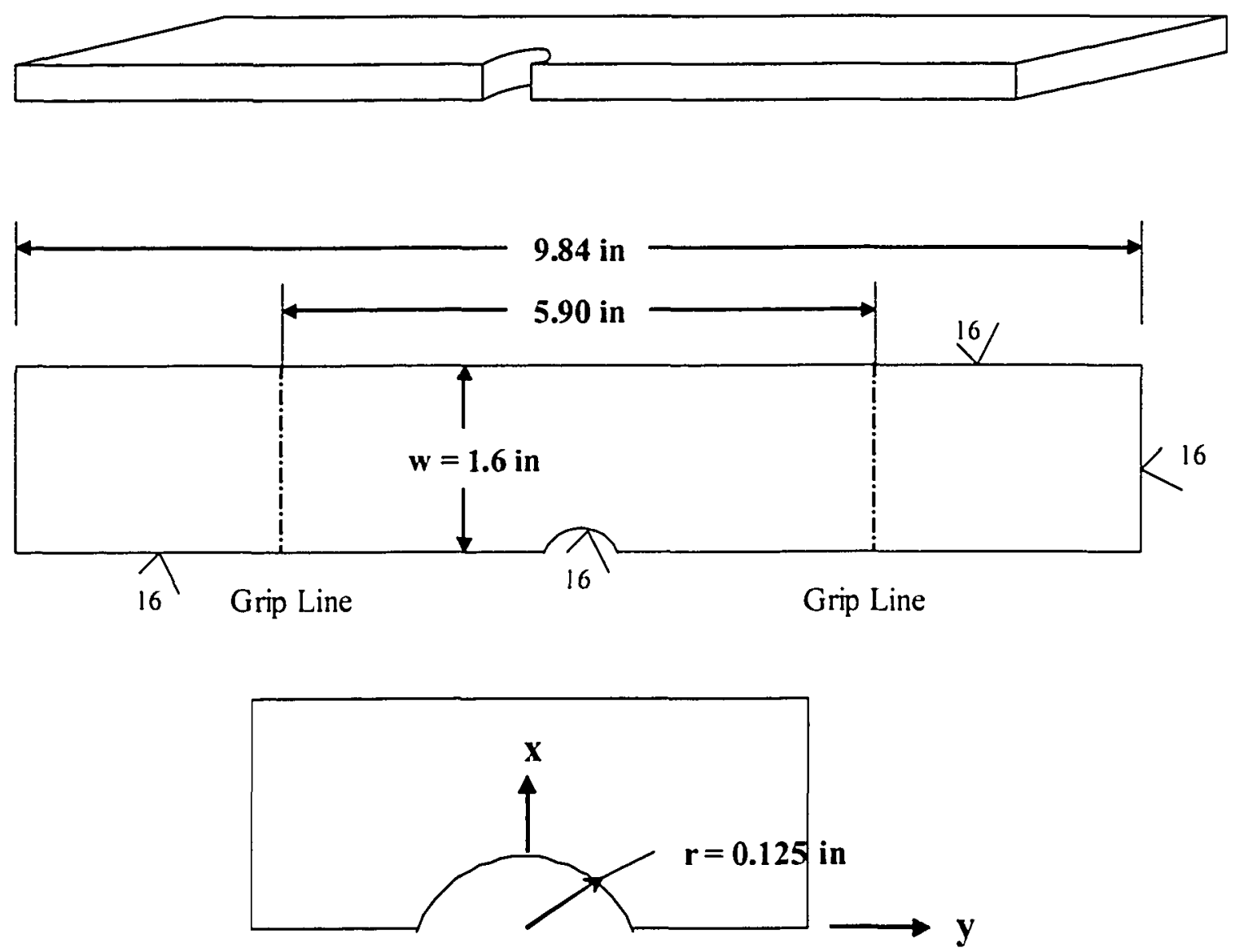

Figure 10. Single-edge notched (SEN) fatigue sample

Standard axial fatigue testing was conducted with the maximum stress levels chosen such that the fatigue life would be between $10^{5}$ to $10^{6}$ cycles. The fatigue tests were 
performed under constant amplitude loading with a constant stress ratio of +0.1 . The fatigue tests were performed at a frequency of $10 \mathrm{~Hz}$ and the specimens were tested so that the loading direction was parallel to the rolling (longitudinal) direction. As a result, the crack front will nucleate and grow on the ST plane.

In addition to the procedures that are presented in this section, a detailed step-by-step procedure for specimen preparation and axial fatigue testing with EPD can be found in the Appendix C and D, respectively.

\subsubsection{Surface Replication}

The replication technique was performed using Struer's quick curing Repliset $\mathrm{Tl}$, which was chosen because the notch wall was a vertical surface. This replication compound had a curing time of four minutes, a claimed resolution of $0.1 \mu \mathrm{m}$ and was capable of being examined by optical and scanning electron microscopy.

Replicas were taken every 25000 cycles during fatigue testing and the notch surface could be examined for small cracks while the test was in progress or examined in reverse order after the test was completed to determine when and where the fatigue crack nucleated. After each interval, the specimen was held at $80 \%$ of the maximum load and the replica compound was applied and allowed to dry on the notch wall. The replica was then removed and the fatigue test was restarted until the next set of replicas was taken. 


\subsubsection{Marker Bands}

A majority of the fatigue samples tested were also subject to series of marker bands every 5000 cycles. The marker bands are distinguishable on the fracture surface because the peak amplitude load has been decreased by $20 \%$. The constant swapping between underloading and peak loading creates dark bands on the fracture surface due to the differing crack growth rates $(\mathrm{da} / \mathrm{dN})$ from the different stress levels applied.

A typical marker band procedure is shown in Figure 11. As can be seen, 5000 cycles were the number of cycles chosen to separate the marker bands because there needed to be a sufficient number of cycles in between the marker bands so they are distinguishable on the fracture surface. However, too many cycles could not be used as it would decrease the chances of having a set of marker bands in the first few grains of crack growth (corresponding to the short crack regime). It was found that the transition between the short crack and long crack regime could be easily surpassed within 10000 cycles, which was the initial cycle count between marker bands. With 10000 cycles in between the marker bands, having a set of marker bands close to the short crack regime became very difficult. However, having only 1000 cycles in between the marker bands made them very hard to distinguish, therefore, 5000 cycles was chosen. 


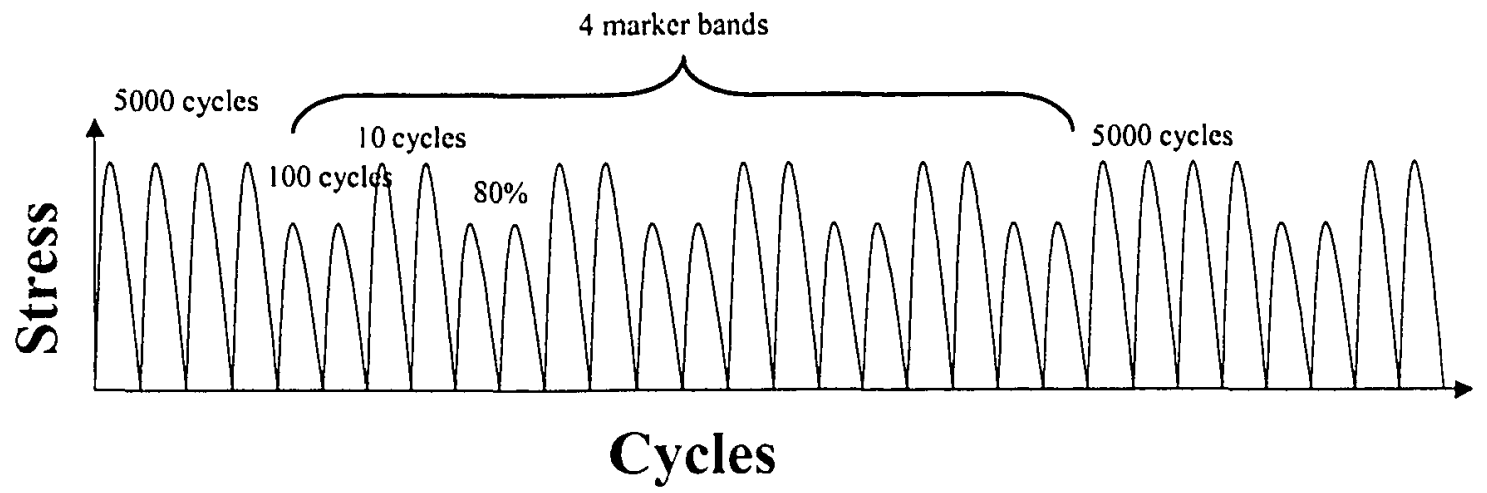

Figure 11. Marker band procedure

In the fatigue testing, rather than having marker bands performed at set cycle counts, the marker bands were implemented at the beginning of the test and repeated every 5000 cycles. A set of marker bands consists of 100 cycles at $80 \%$ of the maximum load and 10 cycles are maximum load. For our fatigue testing, two different sets of marker bands; a $12-2-8$ set and a $6-10-4$ set are used. A $12-2-8$ marker band set refers to a pattern with a set of 12 marker bands, followed by a set of 2 marker bands, and a set of 8 marker bands, all of which were separated by 5000 cycles at maximum load. The $6-10$ - 4 set of marker bands is identical except that it uses a set of 6,10 and 4 marker bands, respectively. A $12-2-8$ set is used for the first three sets of marker bands in order to determine if there was any crack formation and short crack growth within the first $10 \%$ of the fatigue life. After completion of the first three sets of marker bands, the remaining marker bands will be in the sequence of $6-10-4$. 


\subsubsection{Electrical Potential Drop (EPD)}

The electrical potential drop method was performed according to ASTM E647 - 00 . However, the crack growth rate was of no interest in this particular project as the priority was to find a proper detection technique for really small cracks. Therefore, the procedure used closely resembled that found in Annex 6 of the ASTM E647 standard. Figure 12 shows the wiring diagram for the electrical potential drop (EPD) method used to measure crack size. 


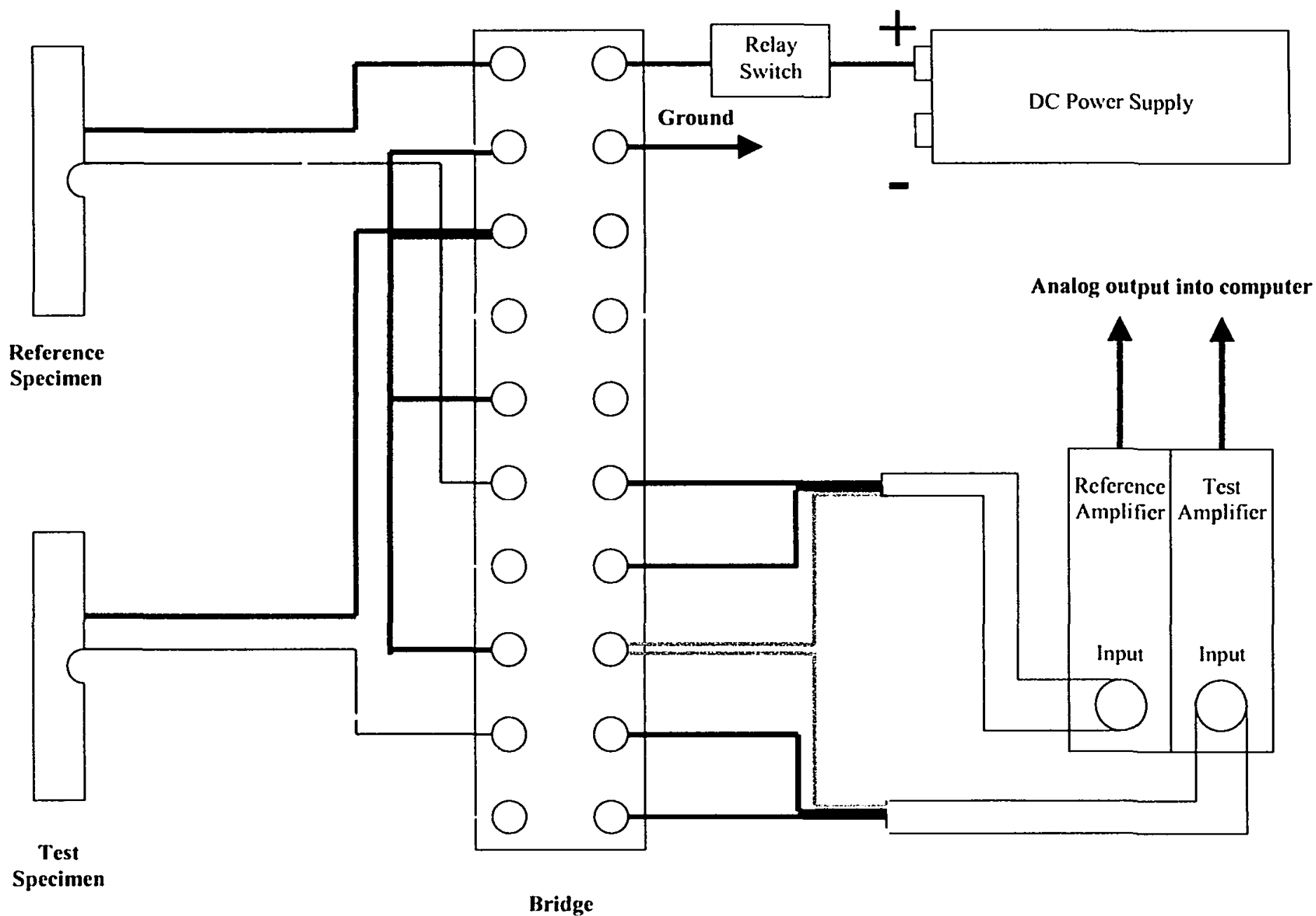

Figure 12. Wiring diagram for EPD crack measurements 
The power to the electrical potential drop testing was supplied by the Lambda LK351FM Regulated Power Supply, which outputs a maximum of $25 \mathrm{~A}$ and $36 \mathrm{~V}$. The two amplifiers used for the reference and test specimen voltage amplification were the Vishay Measurements Group 2310 Signal Conditioning Amplifier, which can amplify a signal up to 11000 times its original strength. These two amplifiers were mounted in the Vishay Measurements Group 2360 Portable Enclosure which can accept up to 4 amplifier modules. Additionally, the voltage readings were being fed from the amplifier into the computer and through the use of the MTS MultiPurpose TestWare (MPT) program, the data was acquired, recorded and exported for subsequent analysis.

The voltage lead wires in the EPD testing were $0.0762 \mathrm{~mm}$. thick ALOMEGA $®$ wire insulated with extruded PFA Teflon $®$. The ALOMEGA $®$ wire is a trademark name for a $\mathrm{Ni}-\mathrm{Al}$ alloy used in thermocouple wiring. The thin $\mathrm{Ni}-\mathrm{Al}$ wiring was chosen for the voltage lead wires because of its weldability to the aluminum test samples. Other types of wiring such as $\mathrm{CHROMEGA} \otimes(\mathrm{Ni}-\mathrm{Cr})$, copper or iron wires may prove difficult to join through spot welding. Spot welding was the preferred option of joining the voltage wires because with the location of these wires so close to the notch that drilling a hole and securing the wires with a screw would have created problems with the failure location being moved from the notch area to where the hole would be drilled. Another reason why such thin wires were selected for the voltage measurement lead wires was because a thin wire allows a more precise location on the fatigue specimen and minimizes the stress on the wire during fatigue testing, which could cause wire detachment. 
These voltage lead wires were welded onto the SEN fatigue sample by the Unitek 1-16303 Spot Welder. With a capacity of $125 \mathrm{~W}^{*} \mathrm{sec}$, the spot welder was more than sufficient for welding on the thin $\mathrm{Ni}-\mathrm{Al}$ wiring. To get adequate fusion between the $\mathrm{Ni}-\mathrm{Al}$ wiring and the 2024-T3 specimen, only $15 \mathrm{~W}^{*} \mathrm{sec}$ of power was required. To ensure the repeatability of the location of the voltage lead wires, a traveling microscope was attached to the spot welder to minimize variation in the location of the lead wires. The voltage lead wires were diagonally across the notch of the sample to obtain average measurements of non-uniform crack fronts.

The current-carrying wiring used in the EPD testing was 22 AWG $(0.635 \mathrm{~mm}$. diameter) copper wiring. The wiring used to carry the current must be very conductive so that it would not heat up significantly and melt during the testing procedure. The 22 AWG wiring can carry 14 A for more than 24 hours without any signs of melting and significant change in electrical properties. $10 \mathrm{~A}$ was passed through the reference and test specimens in series, to make sure that the same amount of current flowed into both specimens, to have an accurate reference reading. A relay switch was inserted into the circuit and controlled by the MTS controller to allow an automated process for interrupting the current before specimen failure. This was important because current flow during failure of the fatigue specimen causes a voltage surge between the two fracture surfaces immediately on failure and damages the amplifier.

Stability tests at 10,12 and 14 A were run for approximately 18 hours. As shown in Figure 13 to Figure 15 , a relatively stable reading was achieved. The main difference 
between the 10,12 and $14 \mathrm{~A}$ levels are the "warm up" times, which is the time required for the readings to become stable. The voltage fluctuations at the beginning are a result of power supply instability and as shown in the results, as the current level is increased, so do the warm up times. At higher than $10 \mathrm{~A}$, the design of the testing procedure would have to be altered to prevent significant heating of the 2024 samples, which ages at relatively low temperatures.

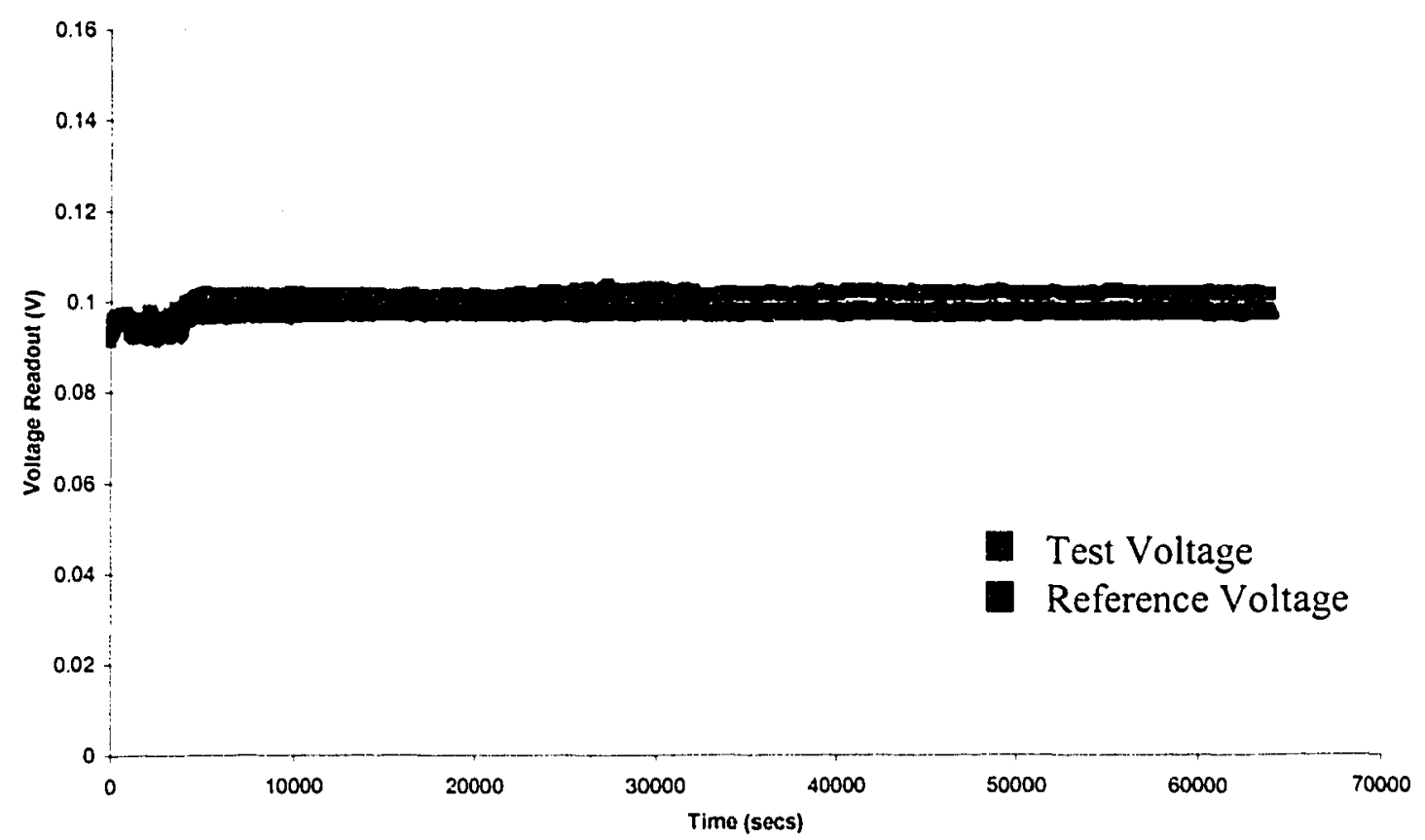

Figure 13. Voltage drop reading stability test at $10 \mathrm{~A}$ of current 


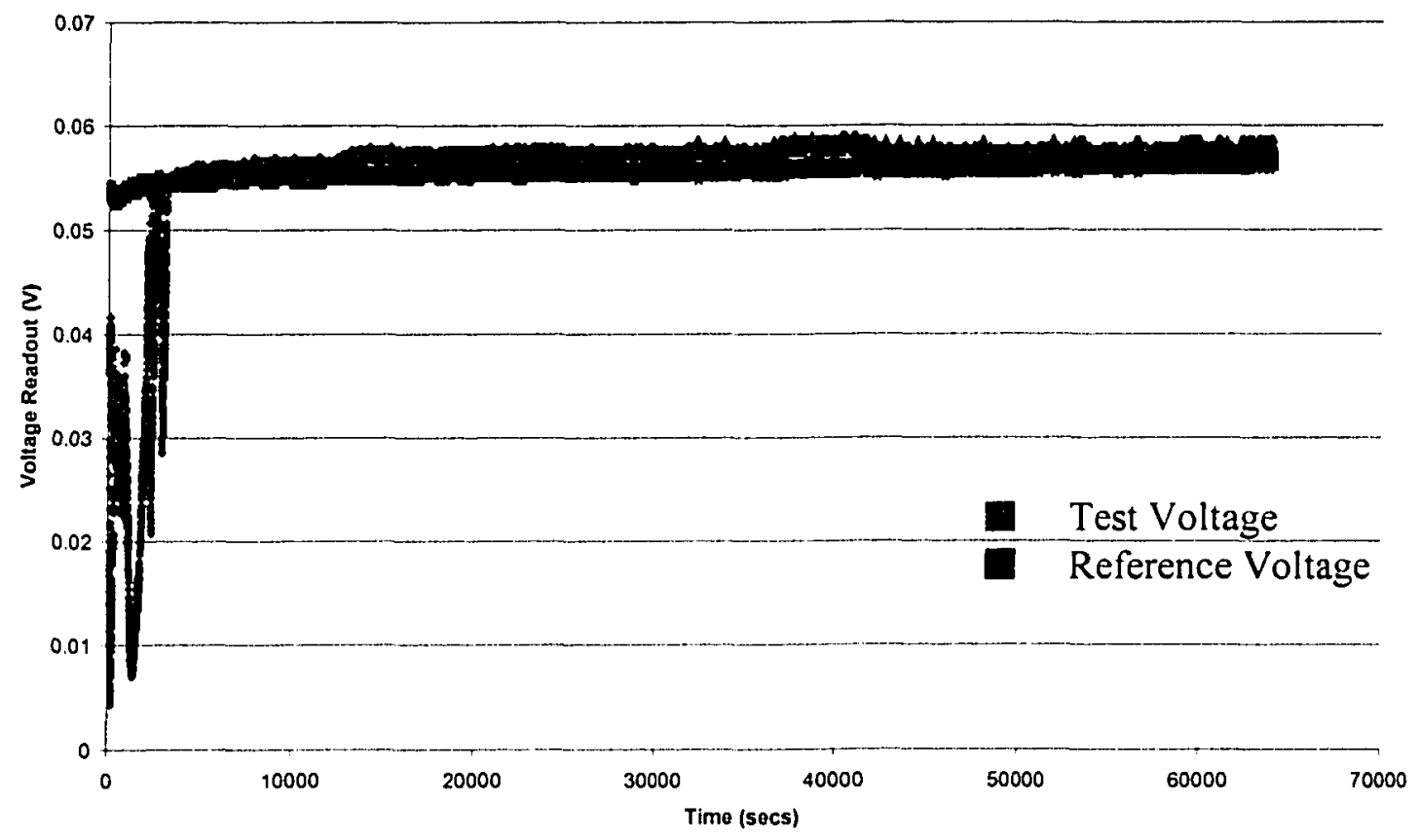

Figure 14. Voltage drop reading stability test at $12 \mathrm{~A}$ of current

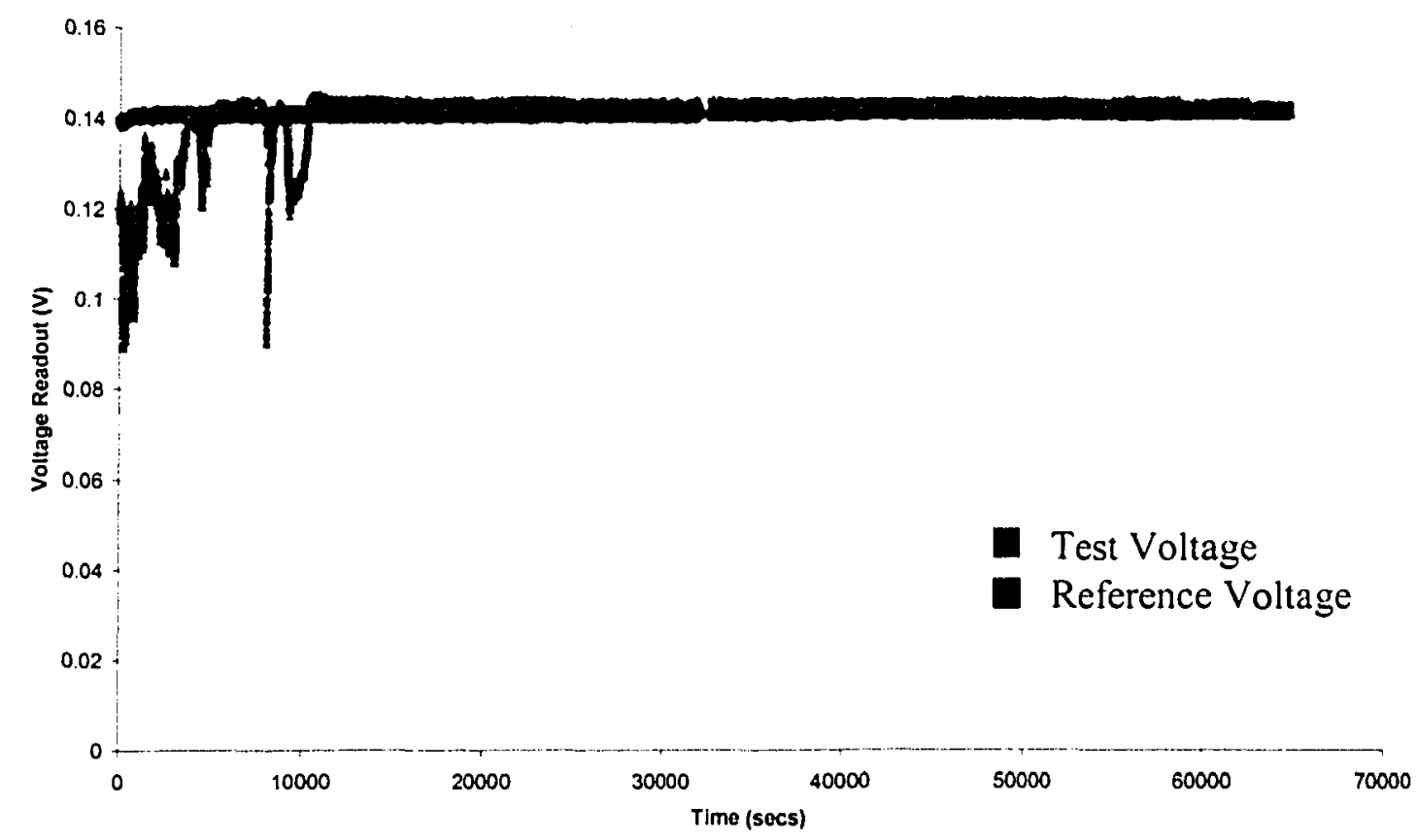

Figure 15. Voltage drop reading stability test at $14 \mathrm{~A}$ of current

A higher current level would be used in cases where the crack size resolution of the EPD data was insufficient. The detectable crack size resolution is mainly dependent of the 
ratio between the initial potential drop output across the notch and the voltage noise level. Because 2024 is a conductive material, the voltage reading will be relatively low and higher current levels will be required to obtain an acceptable level of resolution for crack size detection.

To use a higher level of current in the EPD testing without significantly heating up the aluminum sample, "pulse" readings would have to be taken. Pulse readings involve a relay switch which can handle the higher current output and an alteration of the test design to be able to switch the current on and off. With pulse readings, the current is not flowing constantly through the system; rather, the current is only allowed to flow seconds prior to a couple of readings being taken and is subsequently interrupted after the readings have been recorded, preventing significant specimen heating. Unfortunately, mainly due to time restraints, a relay switch which could handle more than $10 \mathrm{~A}$ could not be acquired. Also, it was found experimentally that the time required for the power source to stabilize, which would produce more accurate readings and minimize experimental errors, in the stability tests run at 10,12 and 14 A (in Figure 13 to Figure 15 ), showed that pulse readings would not be an option, therefore, because pulse readings are not possible with the present setup, a high current level could not be used. The highest current that could be applied without heating up the sample significantly was 10 A and to combat the low voltage readings, the amplification of the voltage readings was increased. However, there are limitations on the amplification of the voltage signal as well. As shown by Figure 16 and Figure 17, increasing the amplification above certain 
levels will cause excess noise and reduce the resolution, making the detection of small cracks very difficult.

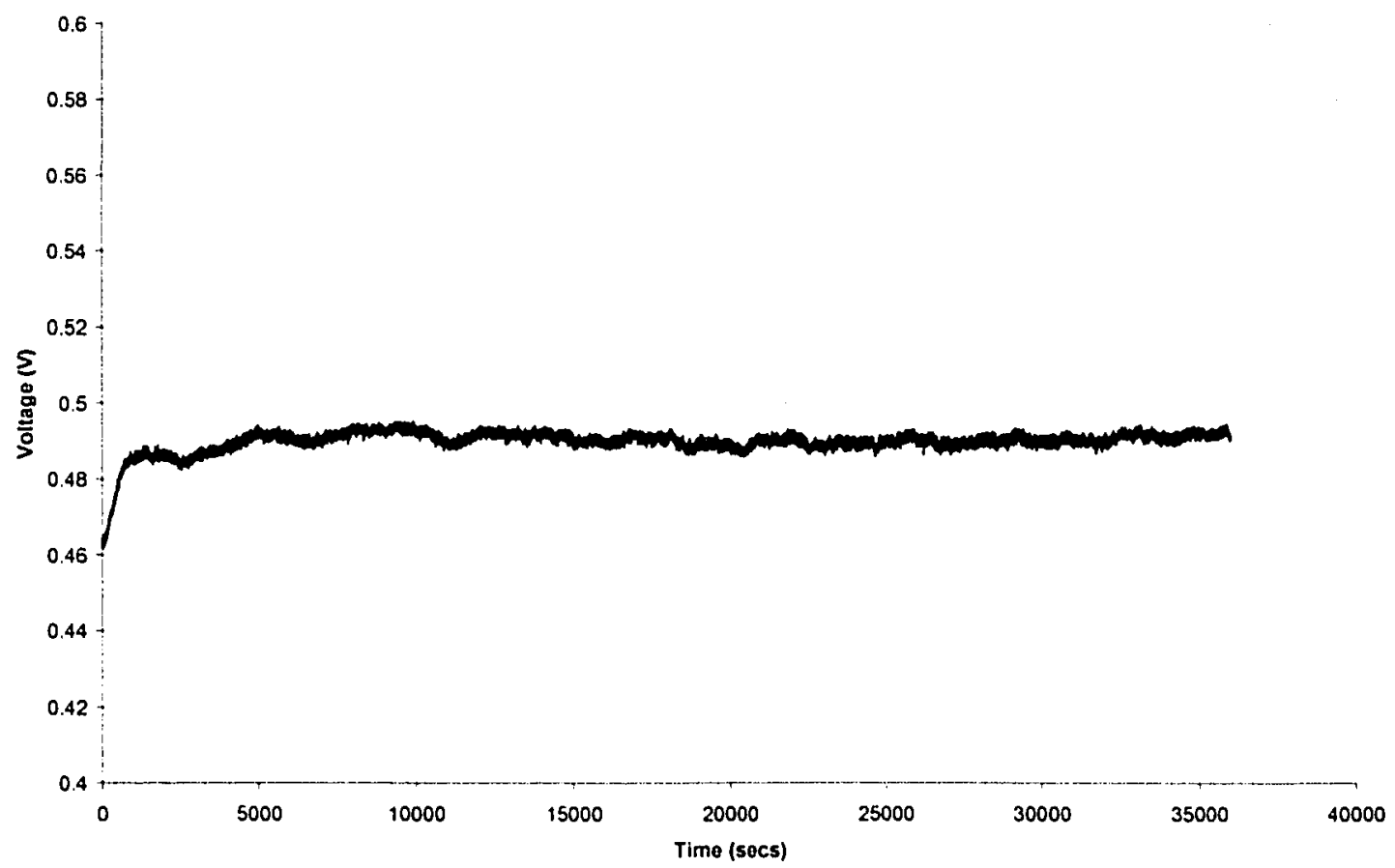

Figure 16. Voltage variation test with an amplification of the voltage signal by $5000 \mathrm{x}$ 


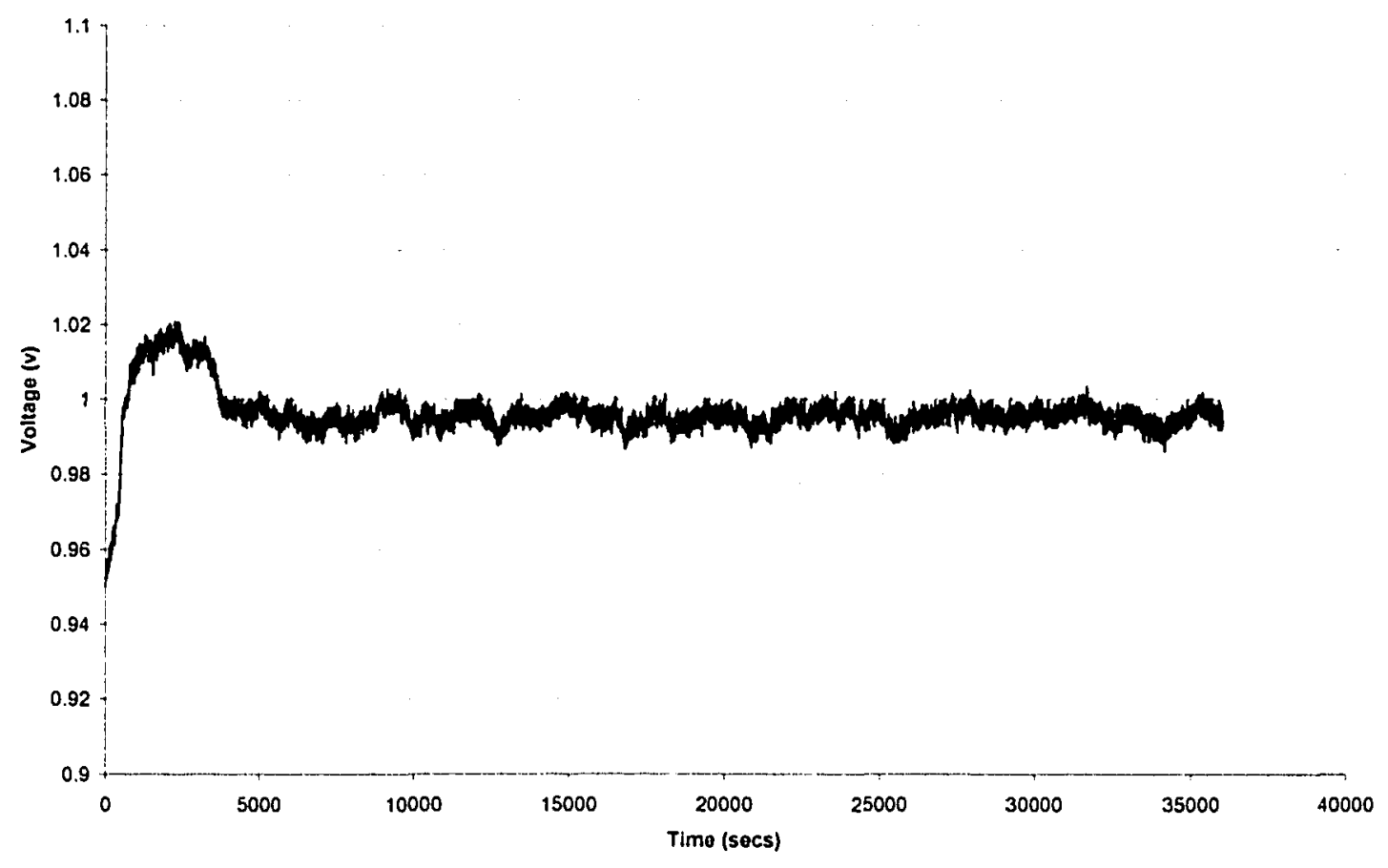

Figure 17. Voltage variation test with an amplification of the voltage signal by $10000 \mathrm{x}$

As it will be explained later, it was found during testing that any sort of voltage reading variation greater than $0.01 \mathrm{~V}$ reduces the resolution below that required for the short crack regime. However, a compromise had to be made because a high amplification was required due to the $10 \mathrm{~A}$ restriction. The $5000 \mathrm{X}$ and $10000 \mathrm{X}$ amplification rates were both tried, but both cases exhibited a poor signal to noise ratio and the crack resolution was not adequate. The problem encountered with the $5000 \mathrm{X}$ amplification gain was that the initial signal reading was too low and the problem with the $10000 \mathrm{X}$ gain was that the noise levels in the signal was too high. Fortunately, it was found that the optimal signal to noise ratio was obtained at $8000 \mathrm{x}$ magnification.

The majority of the post-fracture analysis work performed on the EPD specimens is similar to that for marker band specimens. After a test has been completed, the fracture 
surfaces are examined under scanning and optical microscopy to look for marker bands, which are also applied during EPD testing. The search for marker bands begins starting from failure and working backwards towards the nucleation site to properly match up the cycle count/fatigue performance to each marker band. The distance from the nucleation site to the marker bands is measured and the newly measured crack size is then matched up with the cycle count. The measured potential drop from the EPD testing is outputted versus the cycle count as well. Therefore, the potential drop can be matched up with the crack size. This is how a calibration curve is obtained from EPD testing and a subsequent crack size measuring technique such as marker bands. Once an accurate calibration curve is obtained, this process does not need to be repeated as the potential drop reading can be matched to the proper crack size. 


\subsection{MICROSTRUCTURAL ANALYSIS}

Before any of the fatigue samples were tested, a thorough microstructural examination was performed on the material studied. The following section gives the results from this examination as well as a description of the material and procedures used.

The nucleation of fatigue cracks in 2024 has always been linked to constituent particles and in previous studies [3][4][5], the importance of the first couple of grains adjacent to the fatigue crack nucleation site was established. As a result, the metallographic examination concentrated on establishing the physical and statistical attributes of the grains and the constituent particles.

Figure 18a is a typical optical micrograph showing the etched three-dimensional microstructure in the candidate 2024-T3. Similarly, the typical SEM micrographs of the three dimensional microstructure is shown in Figure $18 \mathrm{~b}$. The SEM micrographs shown were taken using the backscattered electron detector and in these micrographs, the white areas show the constituent particles. The black regions in these micrographs represent the constituent particles that were either partially or completely removed during the polishing or etching stages of metallographic preparation [3][4][5]. Samples of these backscattered micrographs taken in all three planes are shown in Figure 19. 


\subsection{Metallographic Thickness Analysis}

Merati et al. [3][4][5] performed a thickness analysis on 2024-T3 and it was found that the larger particles or clusters of particles were generally found in the centre of the sheet. In order to confirm their findings, a similar thickness analysis was conducted on the candidate 2024-T3. The results for the LS and ST plane are shown in Figure 20 and Figure 21 respectively. As shown in both analyses, there were no significant trends detected and no indications that the larger particles were concentrated in a particular area from comparing the average particle data.

However, when the location of the particles was mapped and the lower limit of the particle area was increased, it revealed that there was a lack of larger particles in the nearsurface region of the plate. This is shown in Figure 22 when the lower limit was increased from a) $0.50 \mu \mathrm{m}^{2}$ to b) $25 \mu \mathrm{m}^{2}$ to c) $75 \mu \mathrm{m}^{2}$ and finally to d) $100 \mu \mathrm{m}^{2}$ for the LS plane. A similar result was found in Figure 23 when the same analysis was conducted for the ST plane. It is believed that the main reason why the larger particles are concentrated in the mid-thickness is because during the hot rolling processes, the strain non-uniformity through the thickness of the plate caused the larger particles closer to the surface to be broken into clusters of smaller particles.

\subsection{Weibull Distribution}

Merati et al. [3][4][5] also found that the particle size distribution followed closely to a Weibull distribution. The particle data collected in all three planes in this project was 
also found to follow a Weibull distribution as illustrated by the linearity of the plots in Figure 24 to Figure 26 for the LT, LS and ST planes respectively. The closer the Weibull plot conforms to linearity, the closer it conforms to a Weibull distribution. The fit of the particle size data to a Weibull distribution is especially strong in the LS and ST planes where the linear trend line has a R-squared value $\sim 0.99$, with a value of 1 being a perfect fit. Figure 27 shows the cumulative Weibull distribution plots for all three planes. As can be seen, the majority $(>95 \%)$ of the particle population is less than $5 \mu \mathrm{m}^{2}$ in size, which means that the larger particles that were found at the nucleation sites make up only a very small percentage of the population and are very rare. Plots of the area distributions (non-cumulative) in Figure 28 to Figure 30 for the LT, LS and ST planes, respectively, show similar results.

\subsection{Microstructural Analysis}

Table 5 and Table 6 show the data that was collected during the optical and scanning electron microscopy of the constituent particles and the grain size. Table 5 also gives a description of each type of measurement. Figure 31 is a graphical representation of some of the data shown in the two aforementioned tables. 
Table 5. Data collected from metallographic analysis of constituent particles

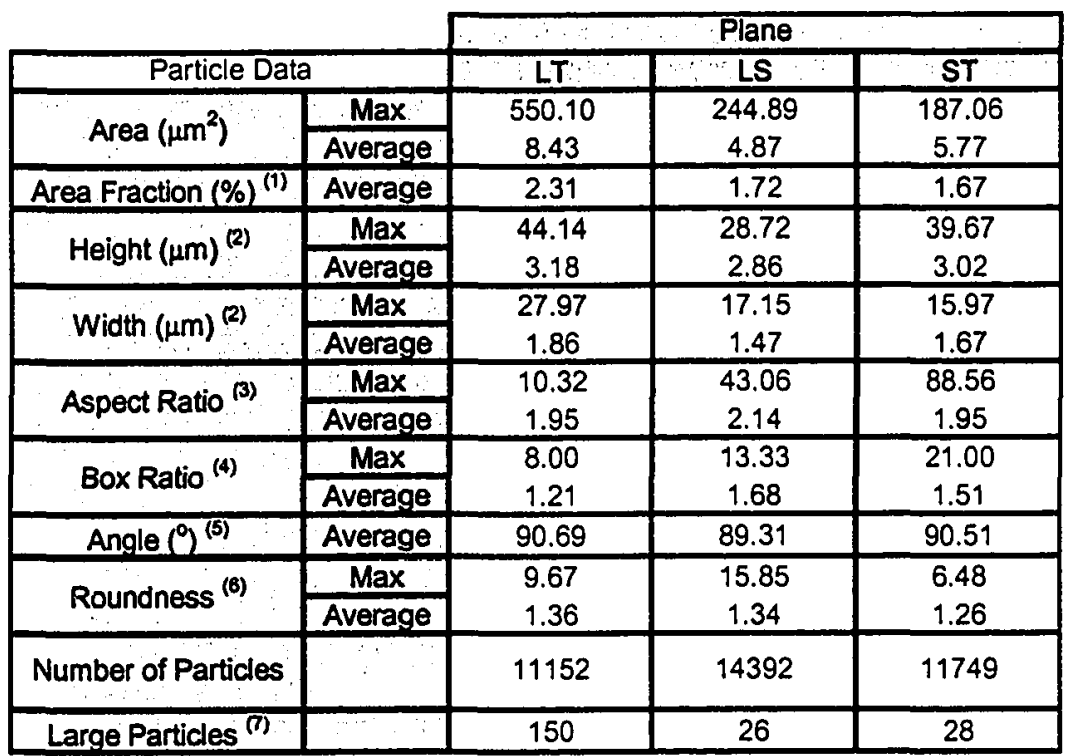

1) Area Fraction (\%) is the cross-sectional area expressed as a fraction of the total area of all particles in the region studied

2) Height and Width correspond to L, T, and S directions according to

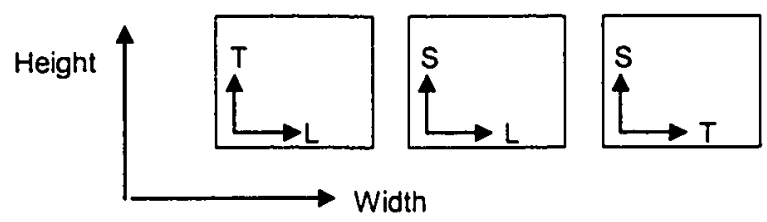

3) Aspect Ratio is the ratio of the longest axis of the particle, regardless of direction, over the longest dimension perpendicular to this axis.

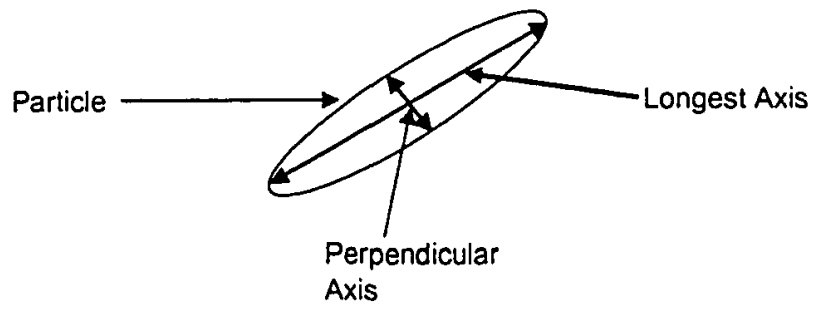

4) Box Ratio is the ratio of the width to height as defined in note 2 above

5) Angle between Vertical Axis (same direction as height) and the longest axis of the particle

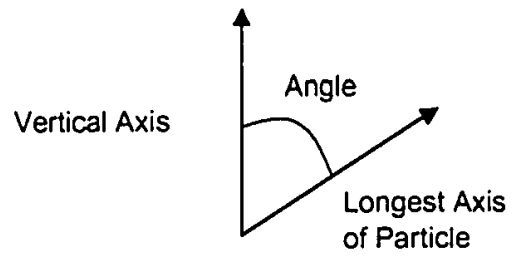

6) Roundness is (perimeter ${ }^{2} /\left(4^{*} \pi^{*}\right.$ Area)). For a circle, the roundness $=1$.

7) Particles with an approximate area greater than $100 \mu \mathrm{m}$. 
Table 6. Dimensions of particles in the $\mathrm{L}, \mathrm{T}$, and $\mathrm{S}$ directions

\begin{tabular}{|c|c|c|c|c|}
\hline & & \multicolumn{3}{|c|}{ Direction } \\
\hline & & $L$ & $T$ & $S$ \\
\hline Grain Size $(\mu \mathrm{m})$ & Average & 234.5 & 115.93 & 33.16 \\
\hline
\end{tabular}

From the data collected, the largest particles, in terms of both maximum size and average size, were on the LT plane. The LT plane had the greatest maximum and average particle sizes in area, height and width. The LT plane also had the highest number of particles greater than $100 \mu \mathrm{m}^{2}$ in size with 150 , compared to just 26 and 28 in the LS and ST planes respectively. This was to be expected since during the rolling process, the particles would be "pancaked" flat on the LT plane, where as on the LS and ST planes, the particles would get "pancaked" longer but also thinner. This was also confirmed by comparing the box aspect ratios of the particles found in the LT plane versus those found on the LS and ST planes. The LT plane has a box ratio of approximately 1.2 where as the box aspect ratios in the LS plane and ST planes are 1.7 and 1.5, respectively.

The grain size of the 2024-T3 was measured using the MaterialsPro $®$ add-on for the ImagePro $\mathbb{B}$ software. As expected, the grain size was found to be the longest in the $\mathrm{L}$ (rolling) direction with a characteristic dimension of $234.5 \mu \mathrm{m}$. The short transverse (S) direction had the smallest of the three directions with a grain size of $33.2 \mu \mathrm{m}$ and the transverse direction had a size of $115.9 \mu \mathrm{m}$. Given that the crack propagated on the ST plane, the two directions of importance are the $\mathrm{T}$ and $\mathrm{S}$ directions. 


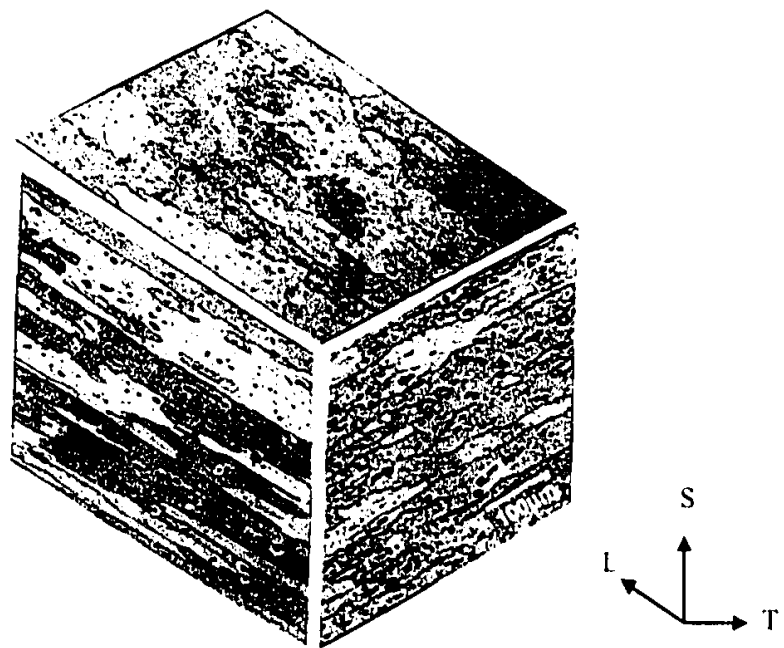

a)

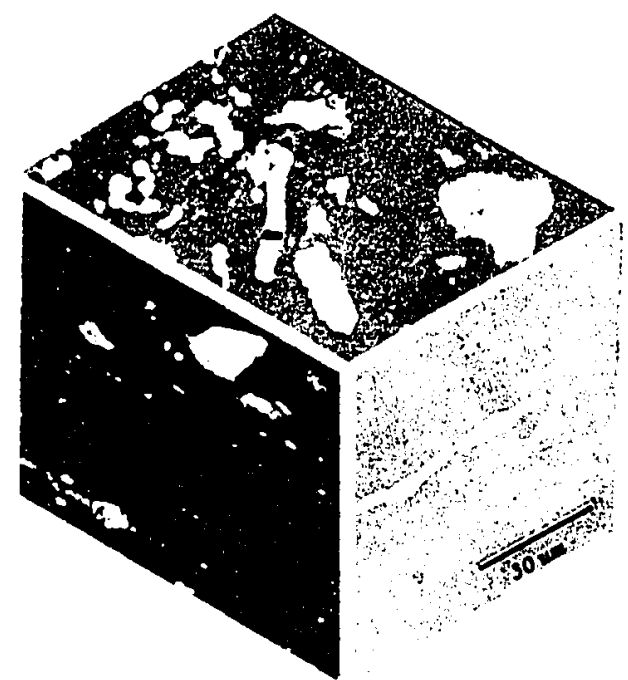

b)

Figure 18. Three dimensional microstructure of the new, bare 2024-T3 aluminum alloy, using a) optical microscopy and b) scanning electron microscopy (backscattered electrons) 

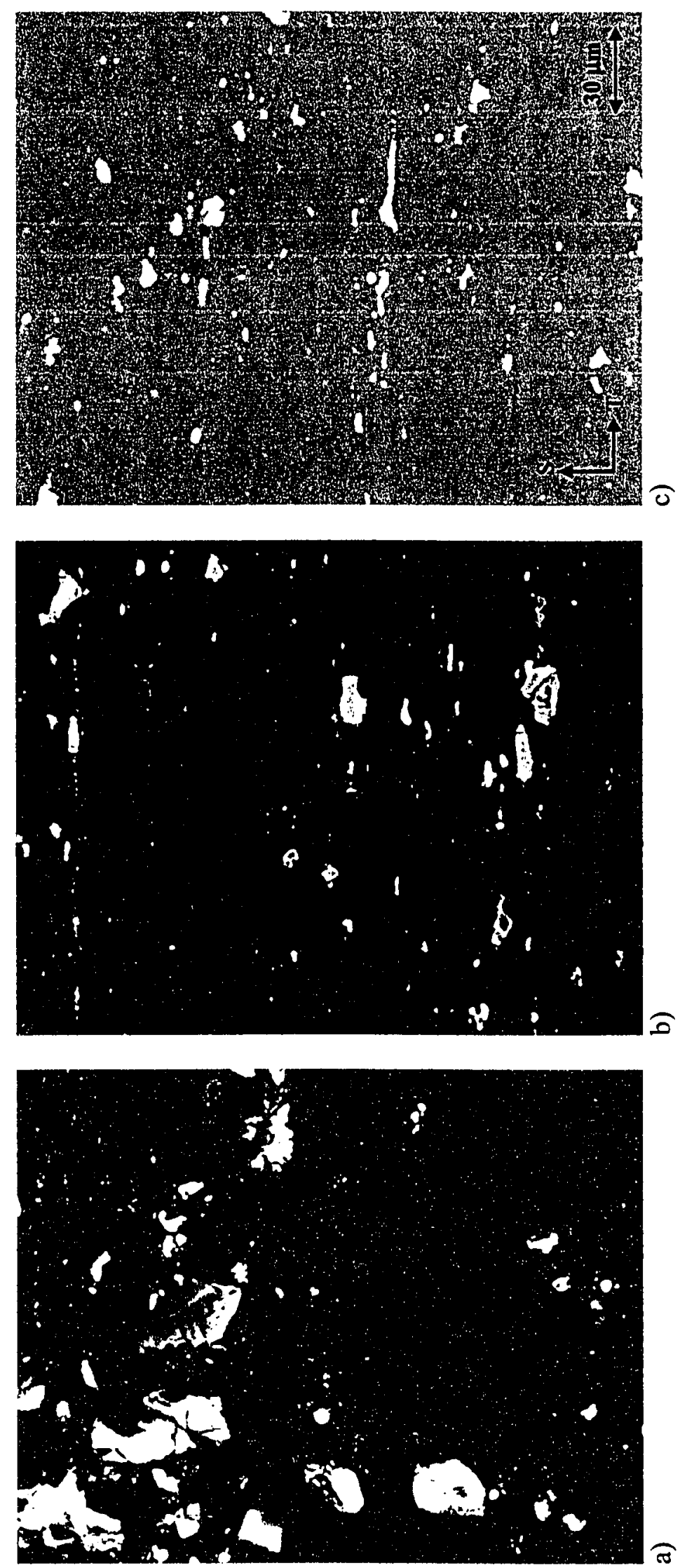

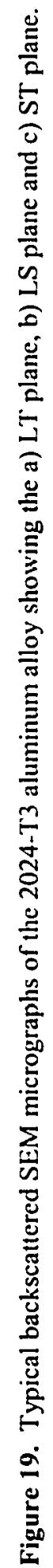




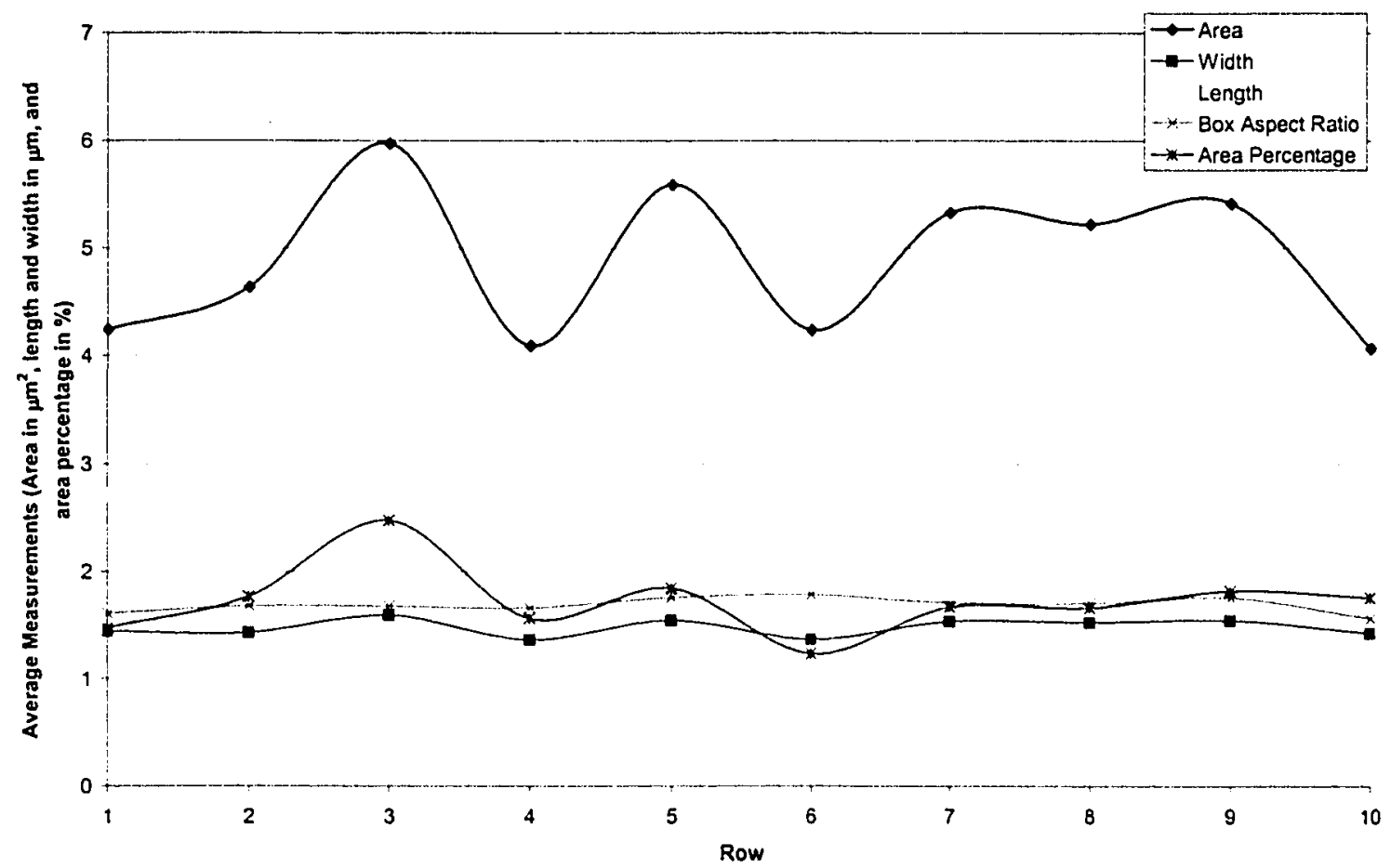

Figure 20. Comparison of the average physical characteristics of the constituent particles throughout the thickness in the LS Plane.

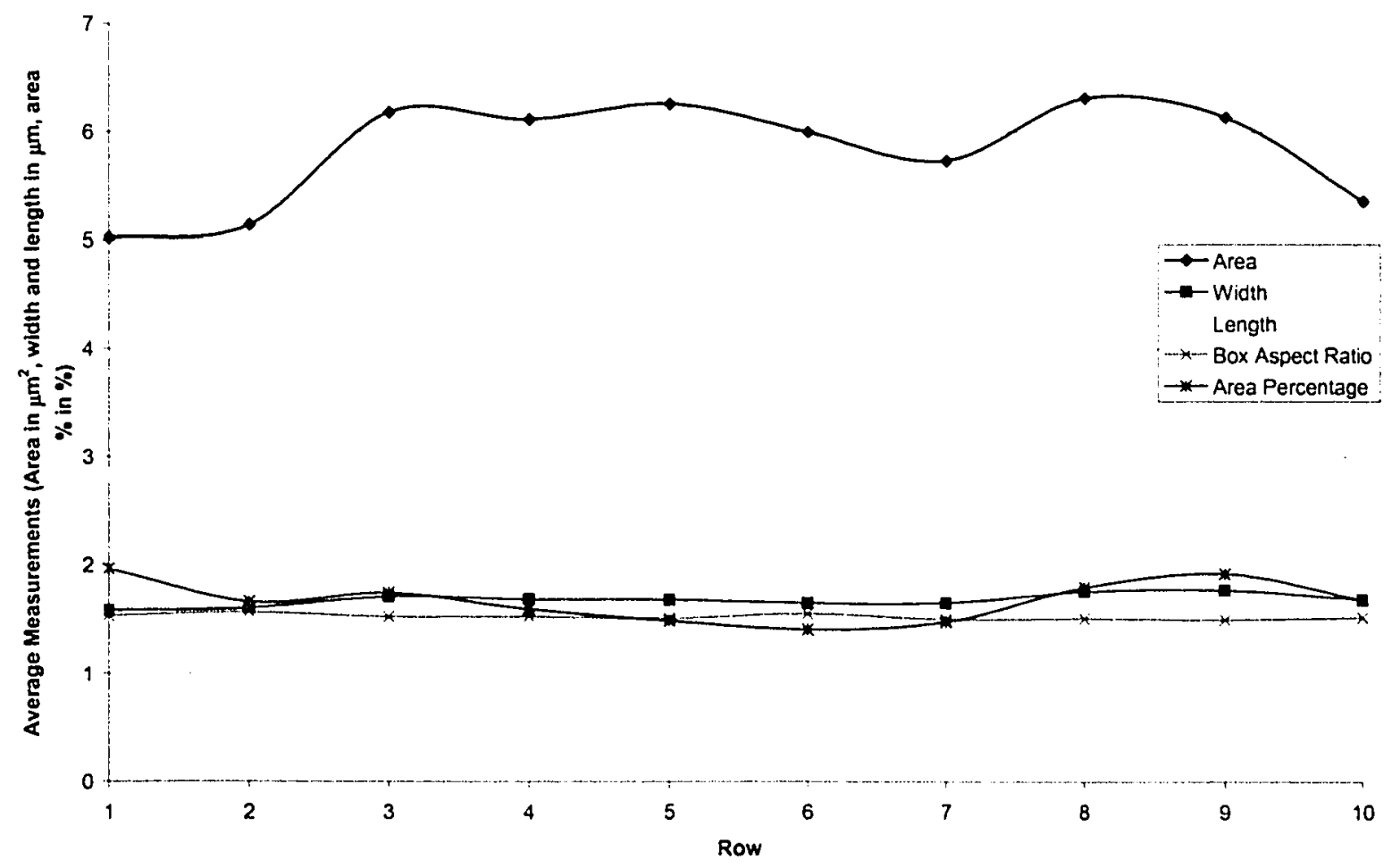

Figure 21. Comparison of the average physical characteristics of the constituent particles throughout the thickness in the ST Plane. 


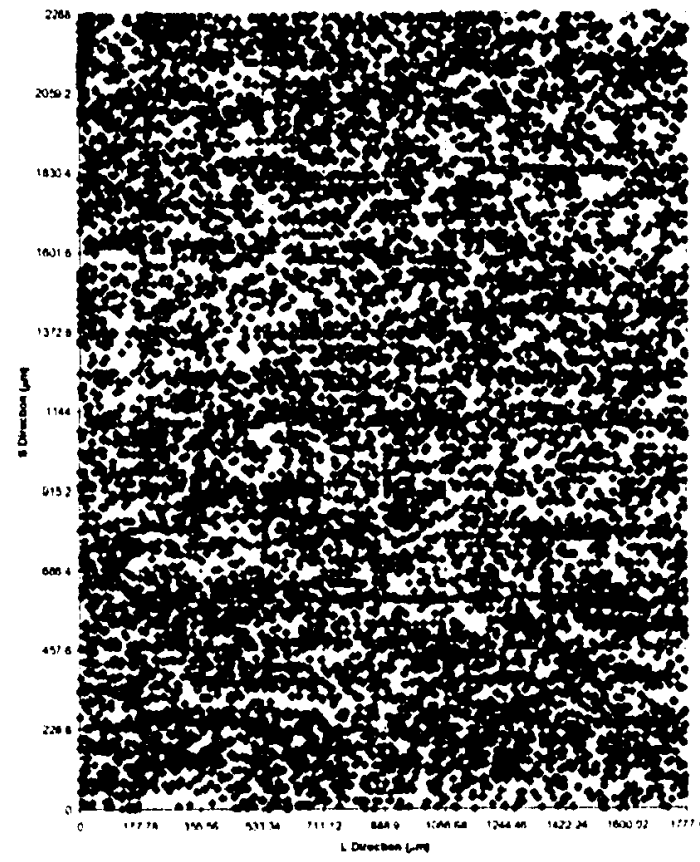

a)

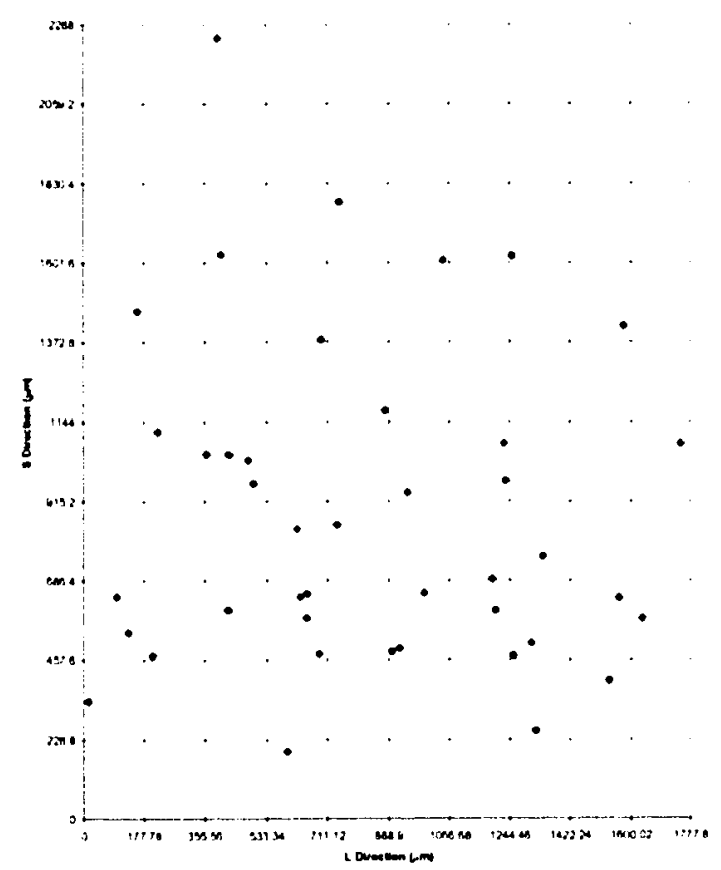

c)

Figure 22. Coordinates of all particles in the LS plane over a) $0.5 \mu \mathrm{m}^{2}$, b) $25 \mu \mathrm{m}^{2}$, c) $75 \mu \mathrm{m}^{2}$, and d) 100 $\mu \mathrm{m}^{2}$.

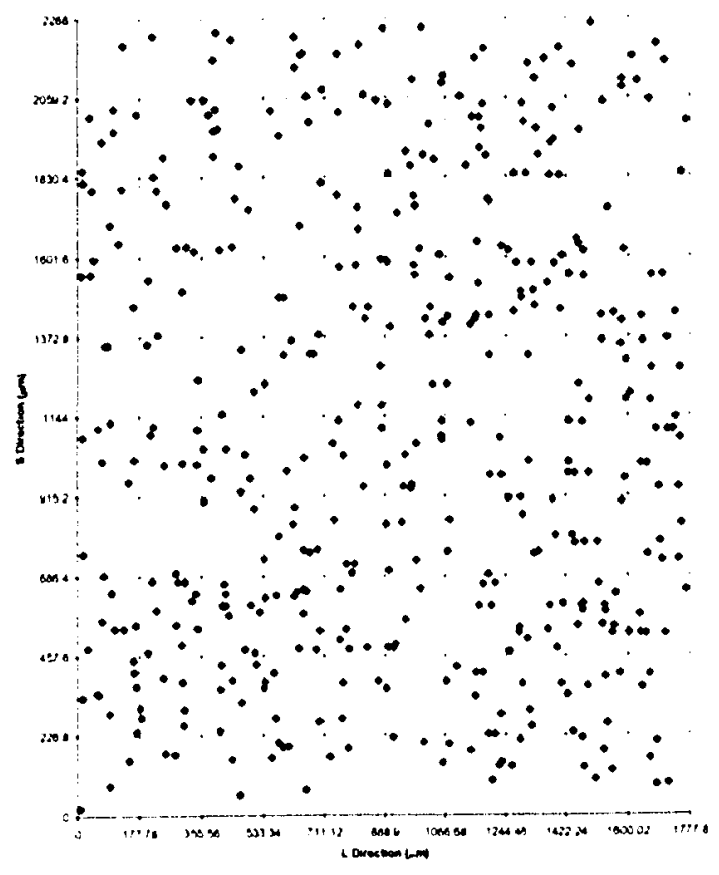

b)

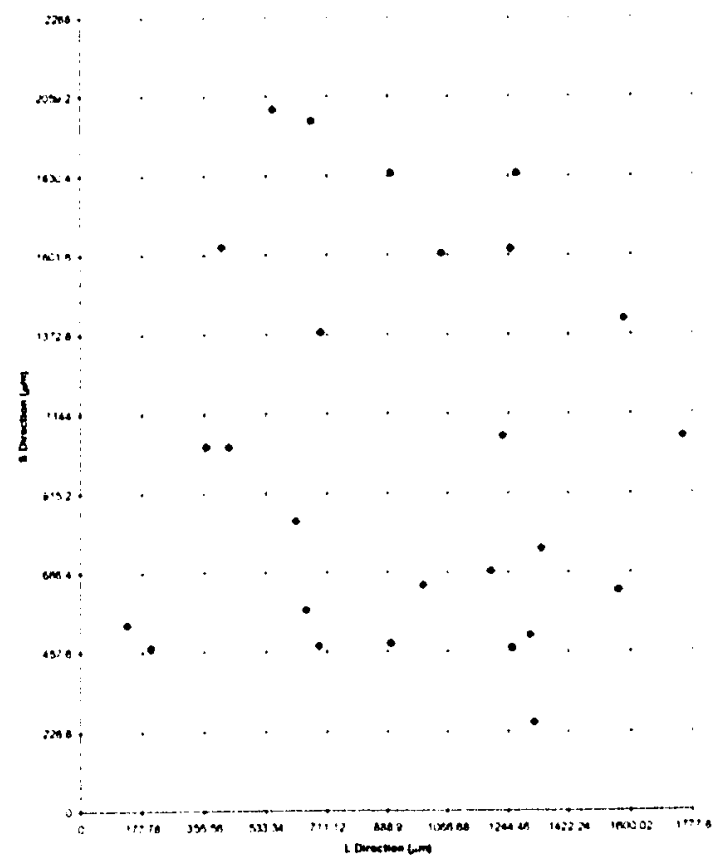

d) 


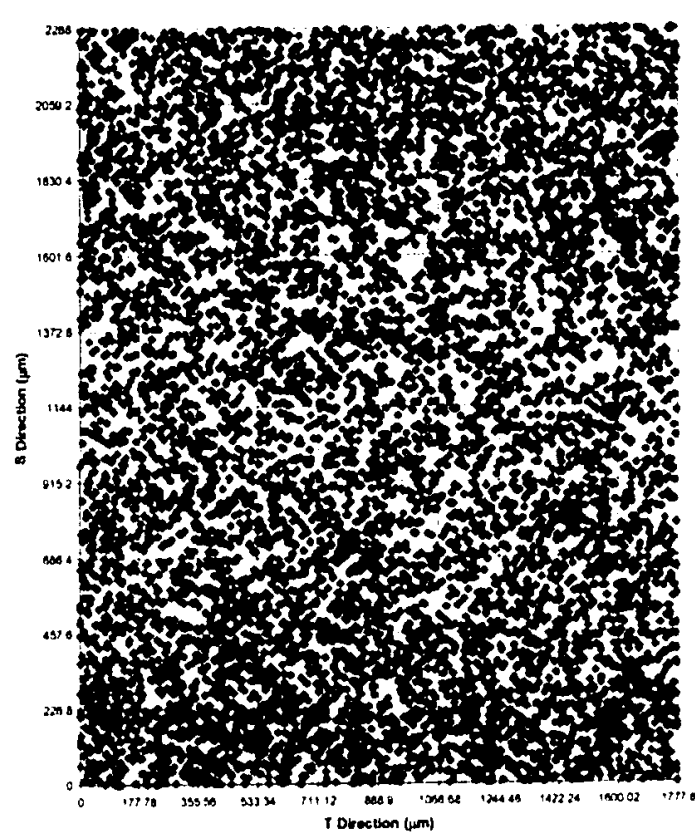

a)

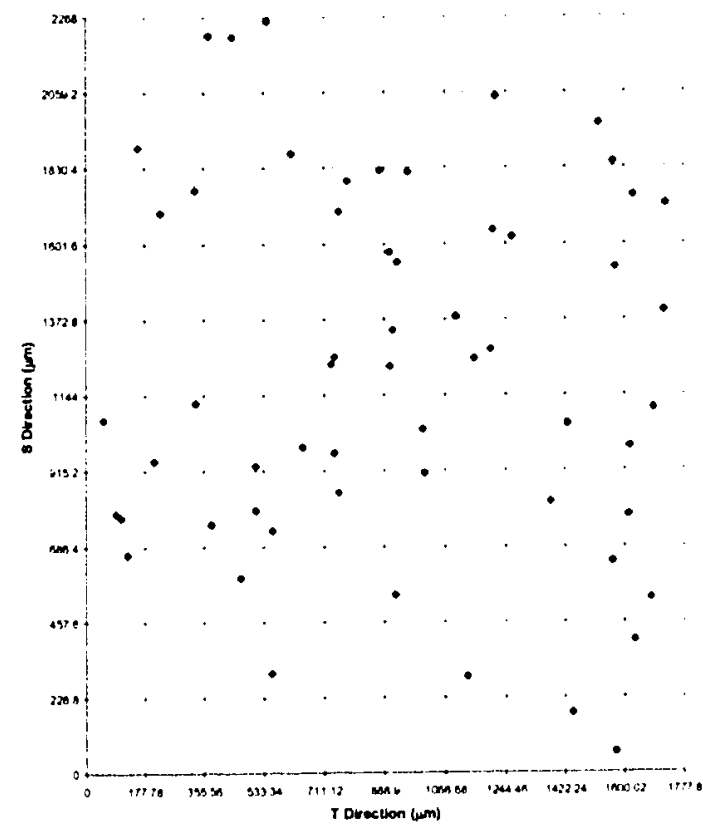

c)

Figure 23. Coordinates of all particles in the ST plane over a) $0.5 \mu \mathrm{m}^{2}$, b) $25 \mu \mathrm{m}^{2}$, c) $75 \mu \mathrm{m}^{2}$, and d) 100 $\mu \mathrm{m}^{2}$.

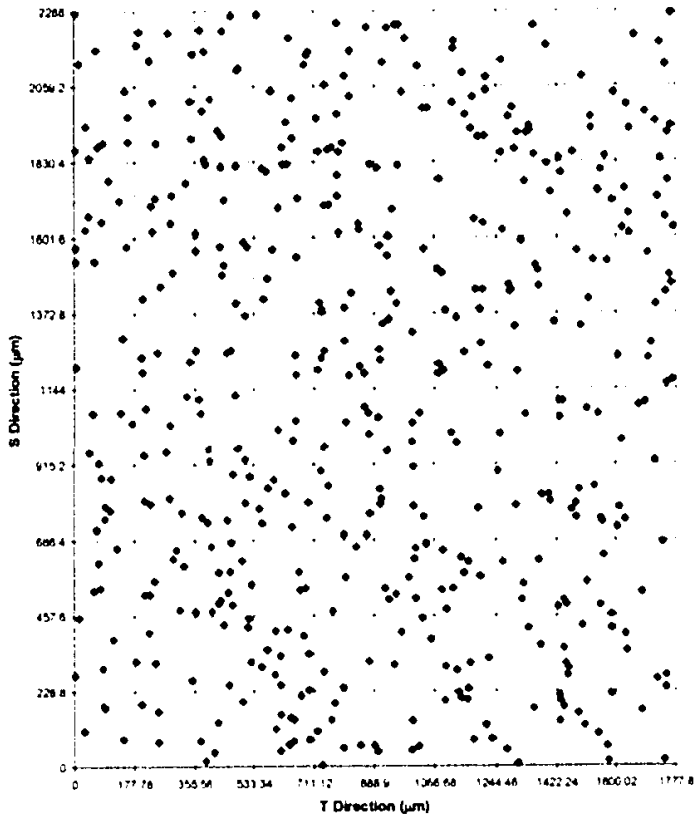

b)

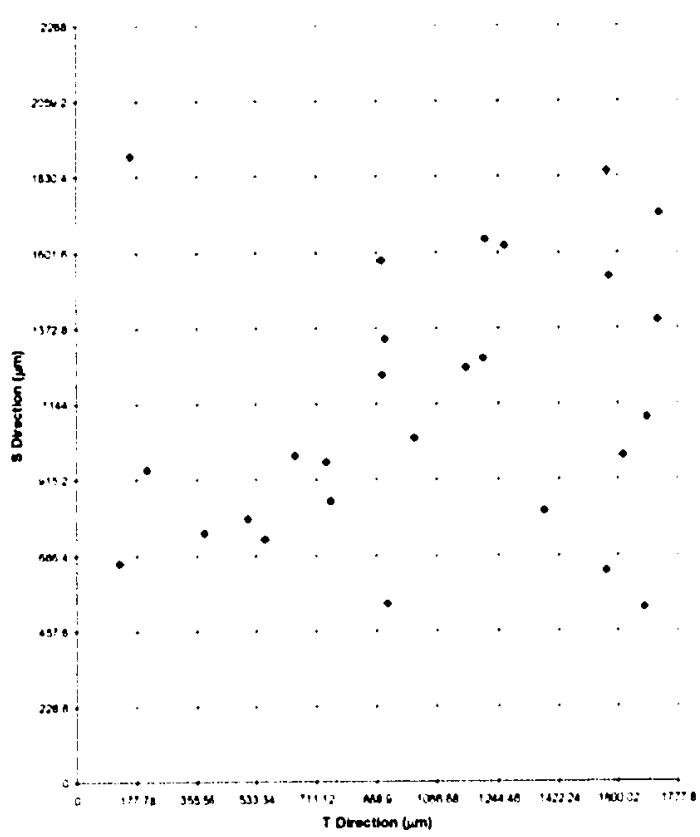

d) 


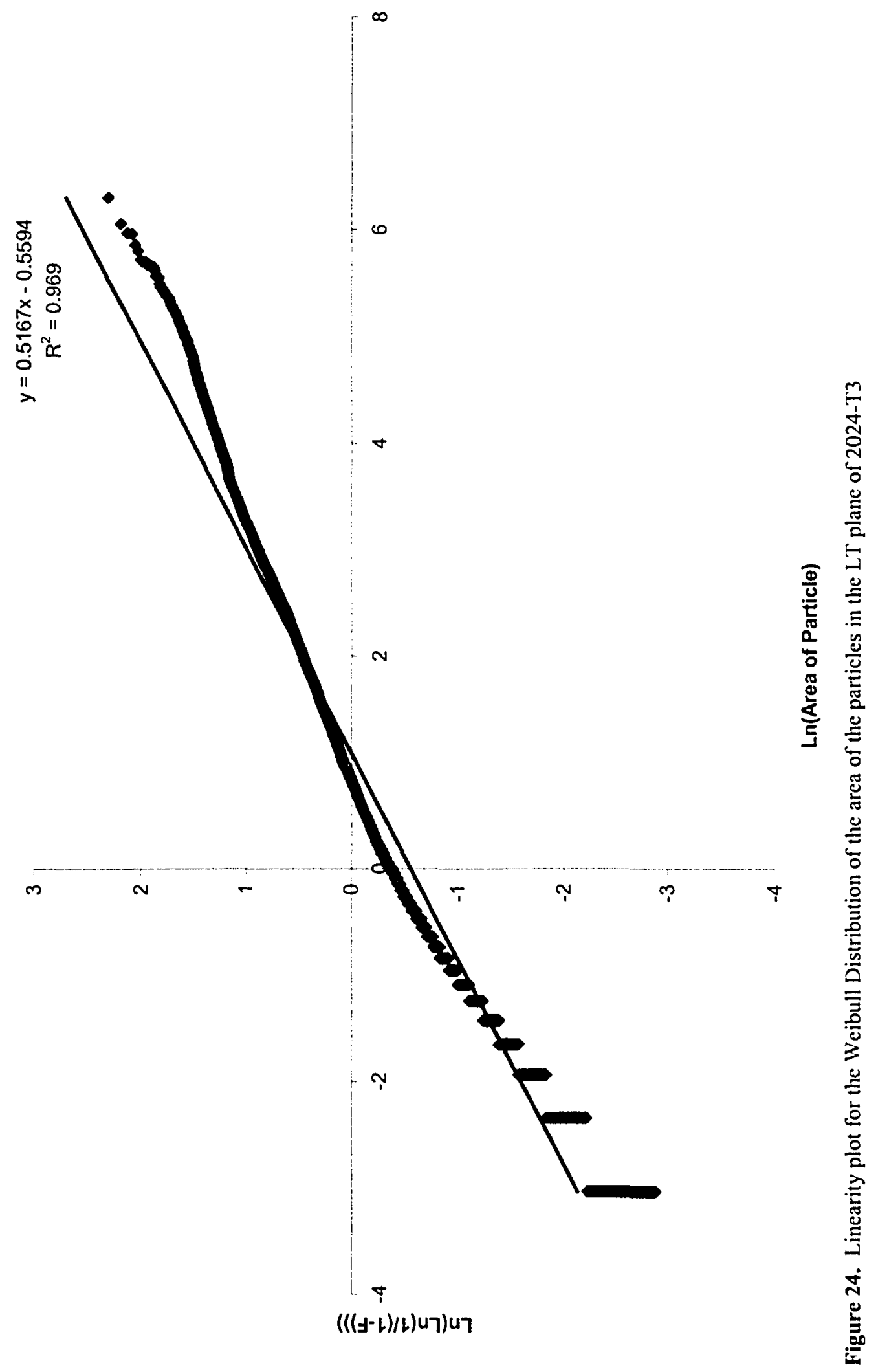




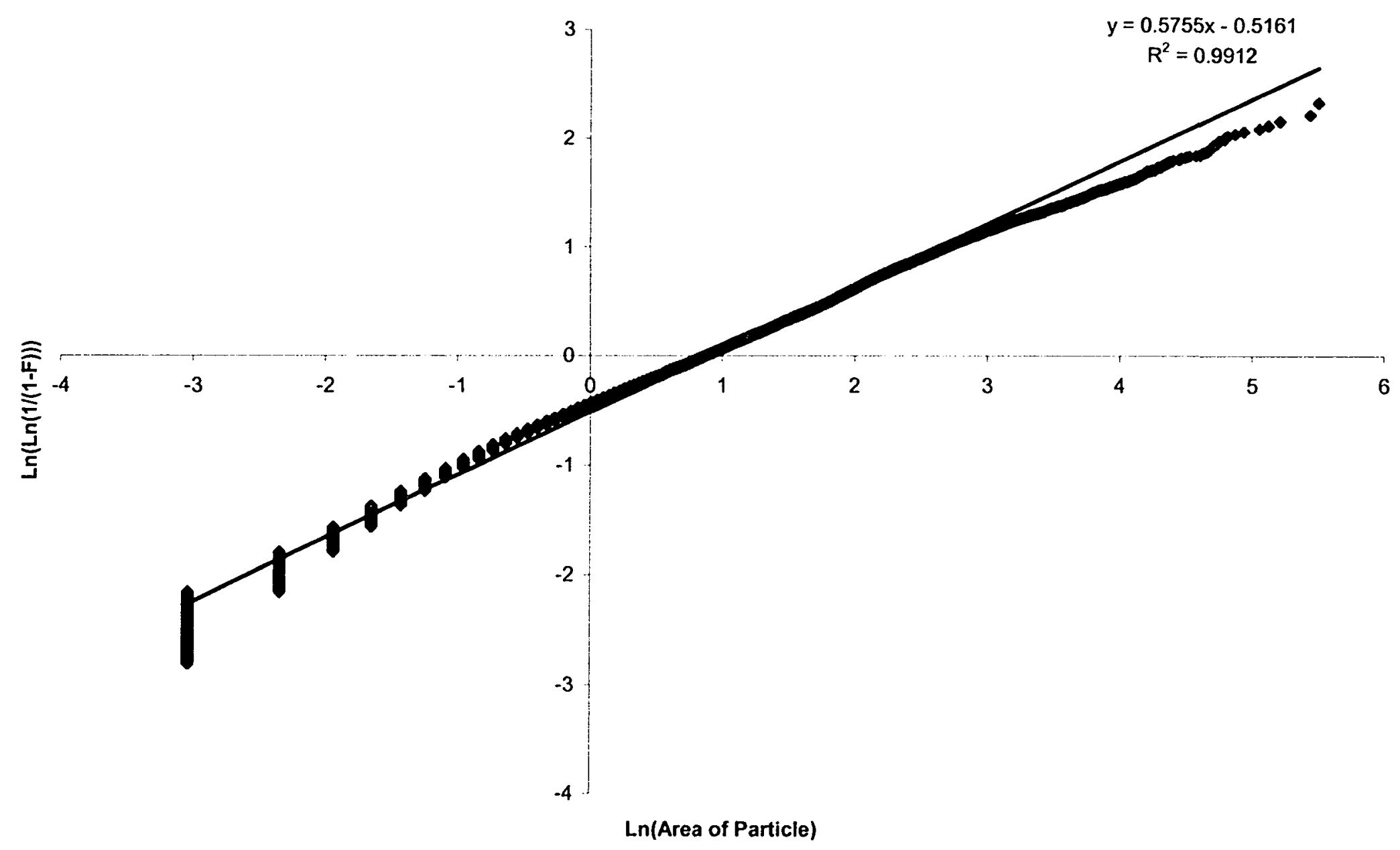

Figure 25. Linearity plot for the Weibull Distribution of the area of the particles in the LS plane of 2024-T3. 


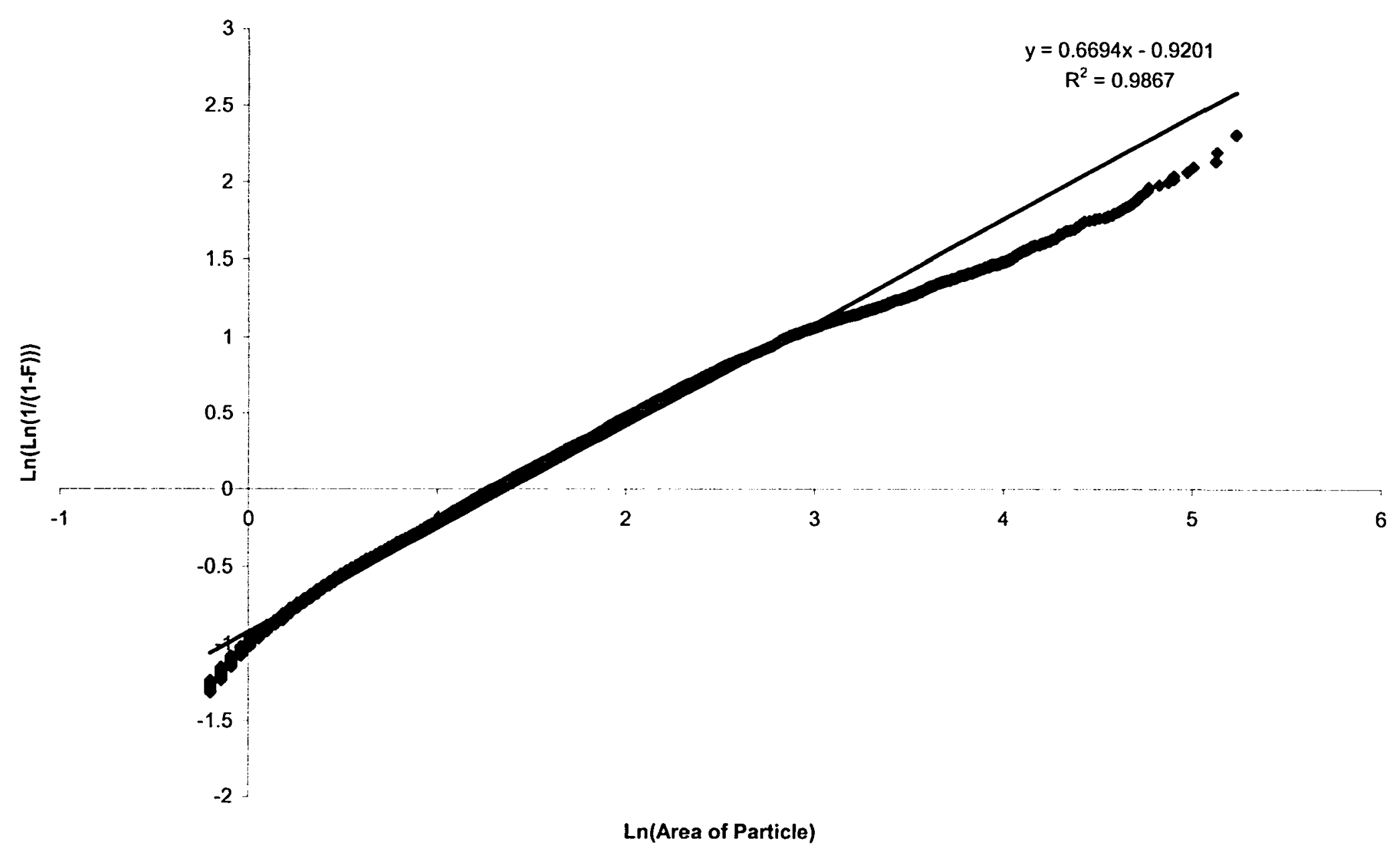

Figure 26. Linearity plot for the Weibull Distribution of the area of the particles in the ST plane of 2024-T3. 


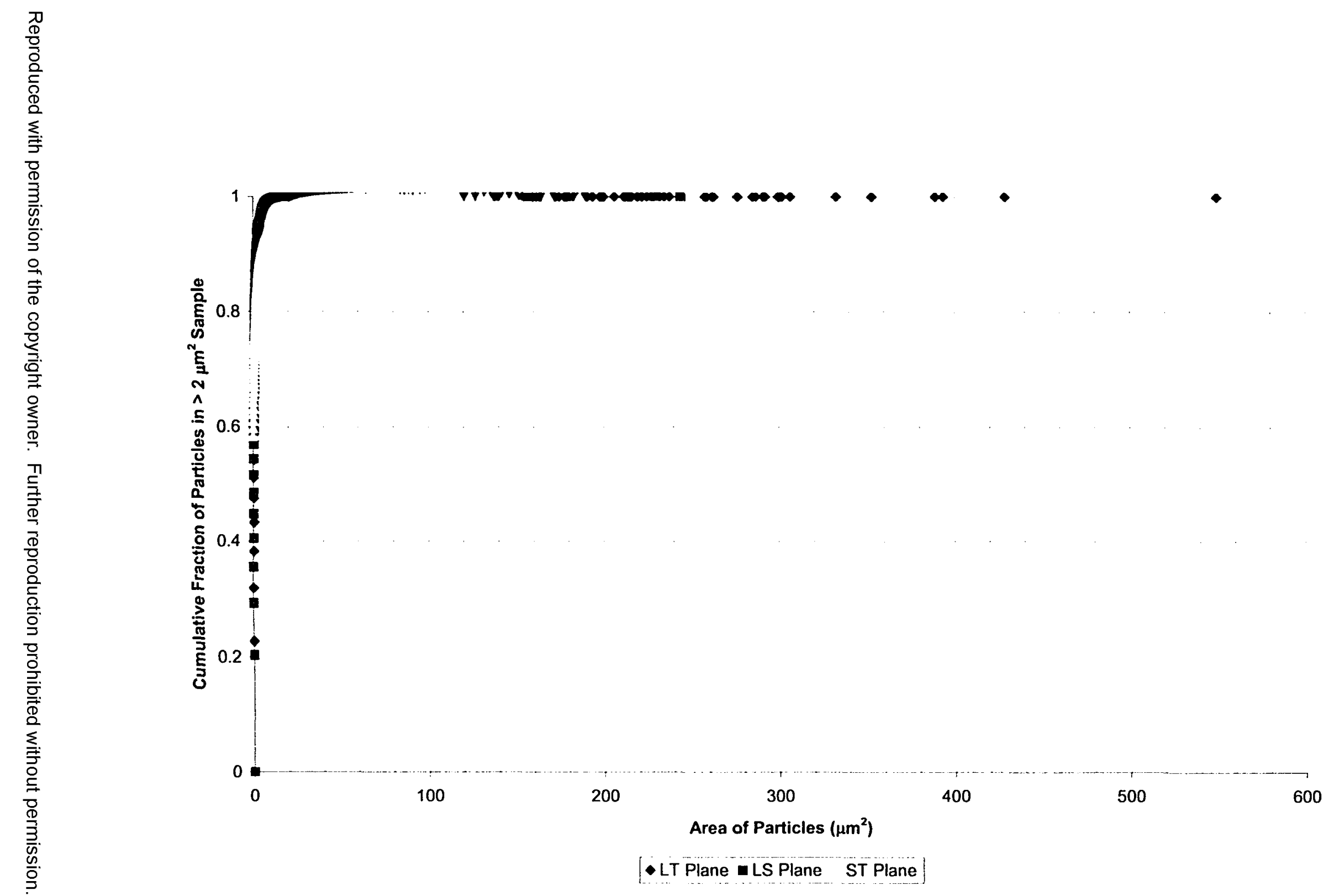

Figure 27. Cumulative Weibull Distributions of the area of the constituent particles in the LT, LS, and ST planes. 


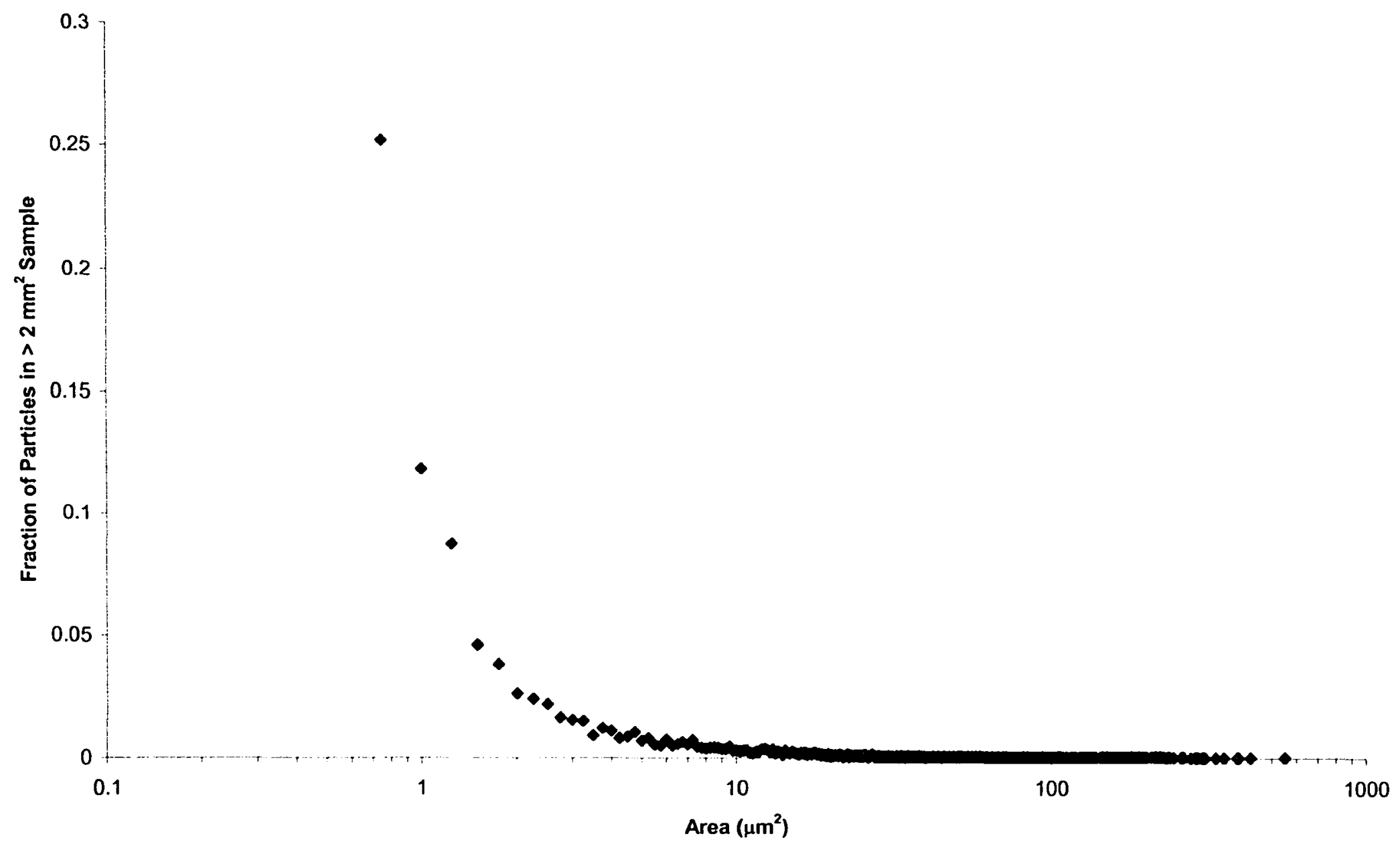

Figure 28. The Distribution of the area of the constituent particles in the LT plane. 


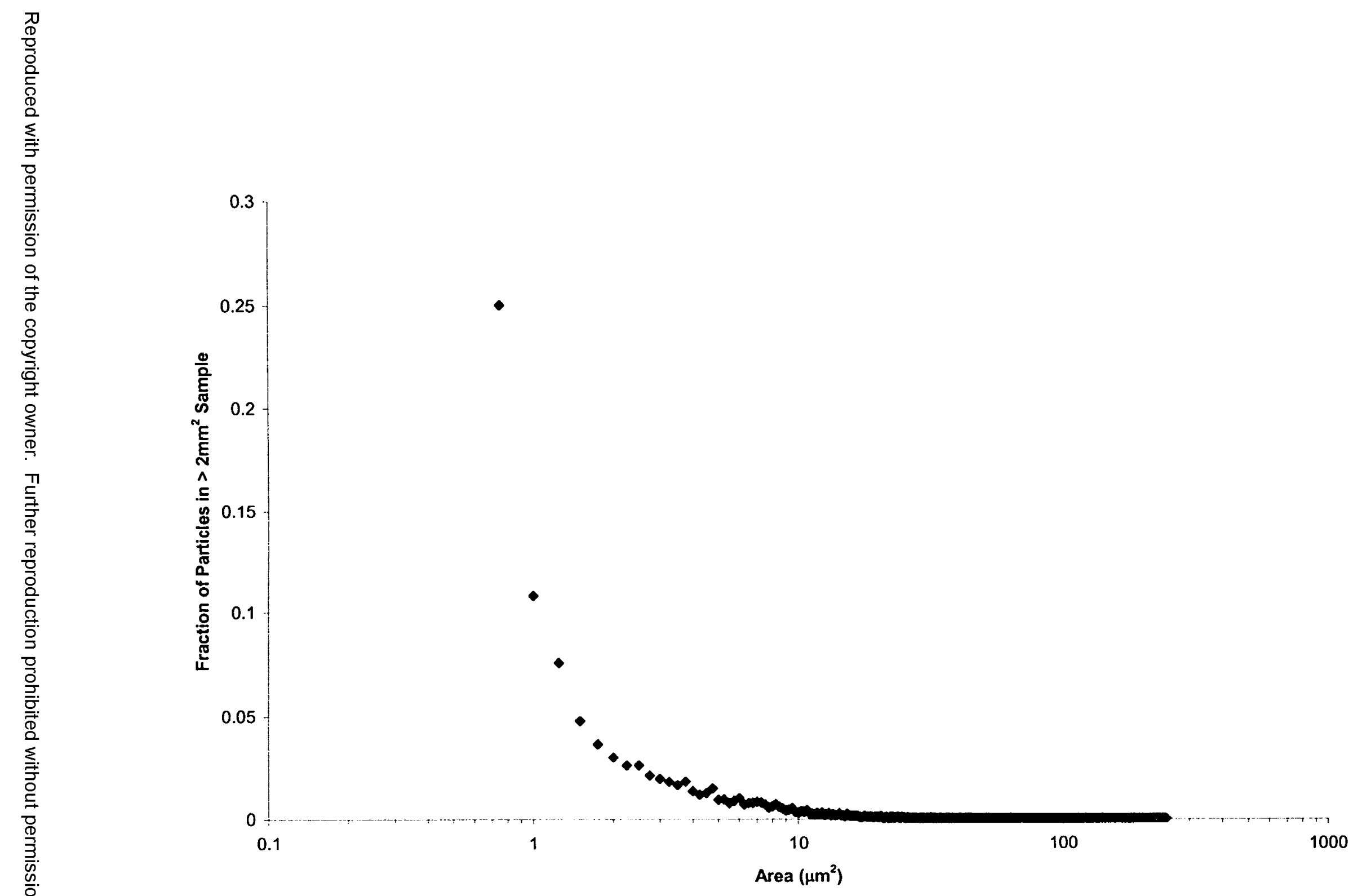

Figure 29. The Distribution of the area of the constituent particles in the LS plane. 


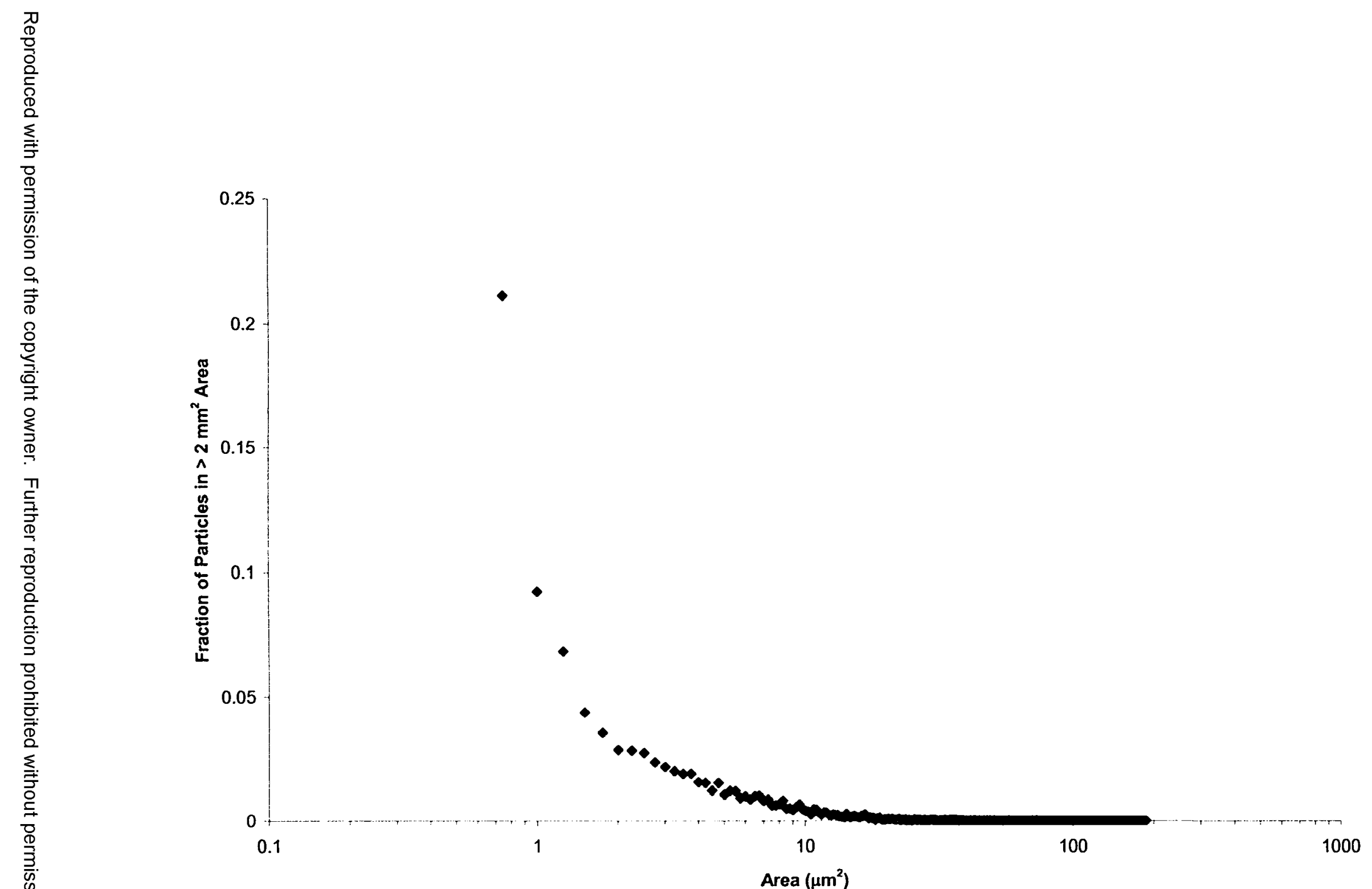

Figure 30. The Distribution of the area of the constituent particles in the ST plane. 


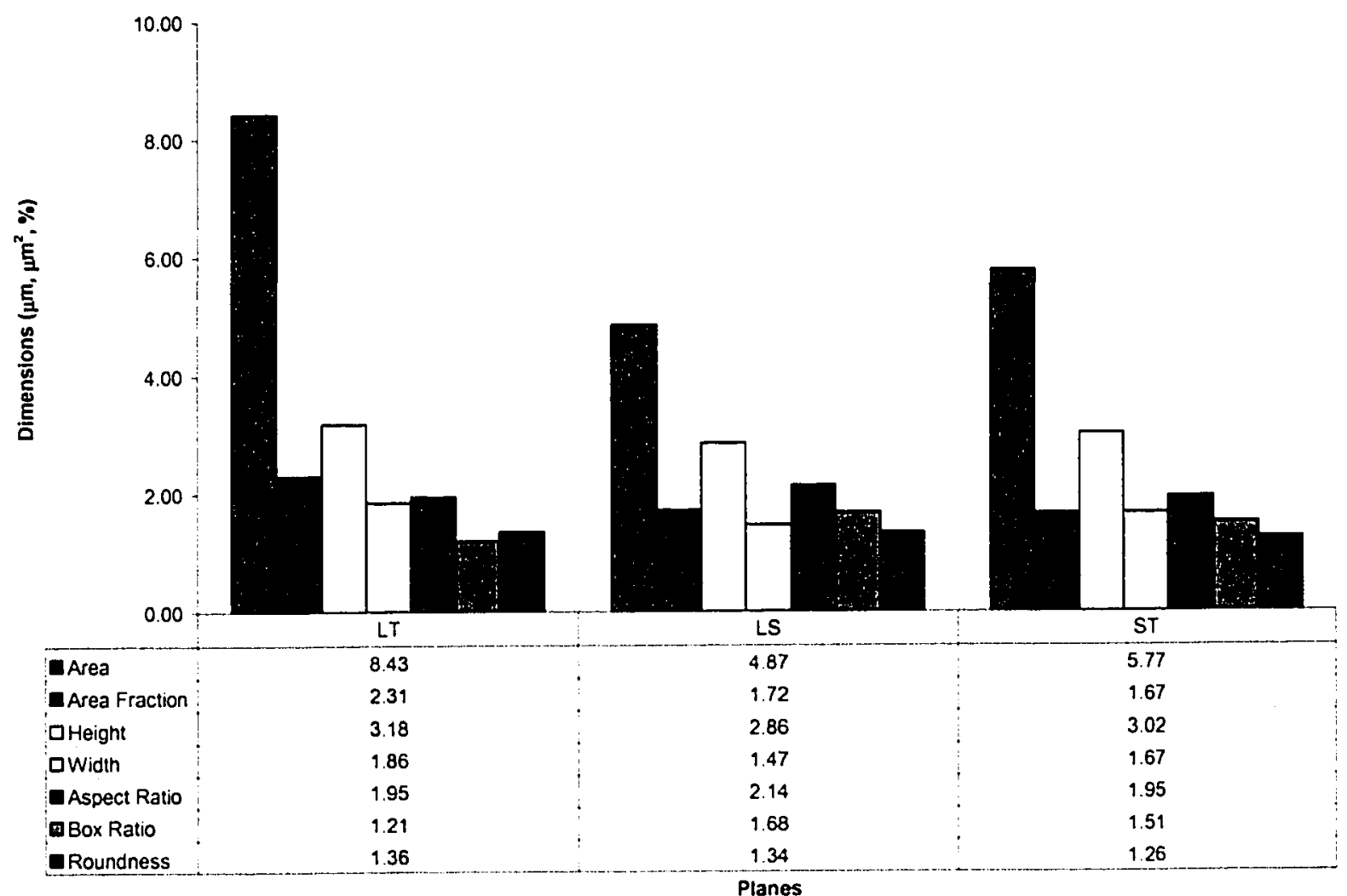

Figure 31. Average dimensions of the constituent particles in the LT, LS and ST planes 


\subsection{RESULTS AND DISCUSSION}

The following section will describe the results obtained from the fatigue testing, postfracture analysis, and EPD testing.

\subsection{Fatigue Testing}

The fatigue results are shown in Table 7 . The results are basically separated by 3 categories based on the max load during testing; $16 \mathrm{kN}$ (3600 lbs), $17.79 \mathrm{kN}$ (4000 lbs), and $21.35 \mathrm{kN}(4800 \mathrm{lbs})$. The average cycles to failure are also included in Table 7 and these results are the average of only the valid test results, which are the ones in the table with a number in the "Result \#" column. All other results were excluded in the average calculations because the samples either weren't tested to failure or failed outside the notch.

In addition, almost all of the EPD fatigue test data was excluded from these results since most of them were not tested to failure. These EPD samples were tested until a crack was detected and then they were pulled in tension to failure so that the crack could be examined in its detected state. 
Table 7. Fatigue Results

\begin{tabular}{|c|c|c|c|c|c|}
\hline $\begin{array}{l}\text { Average Cycles to } \\
\text { Failure }\end{array}$ & $\underset{\#}{\text { Sample }}$ & Result \# & $\begin{array}{l}\text { Load } \\
(\mathrm{kN})\end{array}$ & $\begin{array}{l}\text { cycles to } \\
\text { failure }\end{array}$ & Comment \\
\hline 674828 & $\begin{array}{l}20 \\
19 \\
18 \\
17 \\
\\
16 \\
15 \\
14 \\
13 \\
12 \\
11 \\
\\
10 \\
9 \\
8 \\
7 \\
6 \\
5 \\
\\
21 \\
22 \\
23 \\
24 \\
25 \\
26 \\
27 \\
28\end{array}$ & $\begin{array}{c}1 \\
2 \\
3 \\
4 \\
5 \\
6 \\
\\
7 \\
8 \\
9 \\
10 \\
11\end{array}$ & $\begin{array}{l}21.12 \\
16.59 \\
15.57 \\
\\
16.01 \\
16.01 \\
16.01 \\
16.01 \\
16.01 \\
16.01 \\
\\
17.79 \\
17.79 \\
17.79 \\
17.79 \\
17.79 \\
17.79 \\
\\
17.79 \\
17.79 \\
17.79 \\
17.79 \\
17.79 \\
17.79 \\
17.79 \\
\\
20.02\end{array}$ & $\begin{array}{c}95663 \\
\\
339891 \\
3053154 \\
748099 \\
870733 \\
209088 \\
382456 \\
1000000 \\
1163762 \\
80887 \\
211288 \\
264660 \\
1604421 \\
203033 \\
\\
369154 \\
225873 \\
\\
1752484 \\
2568565 \\
2191239\end{array}$ & $\begin{array}{l}\text { Broke in Handle } \\
\text { Target Max Load was } 16.46 \mathrm{kN} \\
\text { Stopped at } 1000000 \text { cycles and broke in tension } \\
\text { Replica sample with marker bands } \\
\text { Marker band (12-2-8 then } 6-10-4) \\
\text { Marker band (12-2-8 then 6-10-4) } \\
\text { Marker band (12-2-8 then } 6-10-4) \\
\text { Stopped after } 198847 \text { cycles because crack found } \\
\text { Changed to } 5000 \text { cycles between marker bands rather than } \\
\text { 10000 } \\
\text { Broke in Handle (New, larger grips used) } \\
\text { Broke in Handle } \\
\text { Stopped at } 1752484 \text { cycles and broke in tension } \\
\text { Broke in Handle } \\
\text { Stopped at } 2568565 \text { cycles and broke in tension } \\
\text { Increased load }\end{array}$ \\
\hline
\end{tabular}




\begin{tabular}{|c|c|c|c|c|c|}
\hline & 29 & & 21.35 & & Broke in Handle \\
\hline & 30 & & 21.35 & 1867806 & Increased load \\
\hline & 31 & & 21.35 & & Broke in Handle \\
\hline & 33 & 16 & 21.35 & 141279 & Alignment re-done \\
\hline & 36 & 17 & 21.35 & 145439 & \\
\hline & 37 & 18 & 21.35 & 193175 & \\
\hline & 34 & 19 & 21.35 & 110775 & \\
\hline \multirow[t]{25}{*}{182721} & 35 & 20 & 21.35 & 123164 & \\
\hline & 38 & 21 & 21.35 & 154593 & \\
\hline & 39 & 22 & 21.35 & 142891 & \\
\hline & 32 & 23 & 21.35 & 168756 & \\
\hline & 40 & 24 & 21.35 & 331226 & \\
\hline & 41 & 25 & 21.35 & 204963 & \\
\hline & 88 & & 21.35 & 186932 & Broke in drilled hole \\
\hline & 89 & 26 & 21.35 & 188478 & \\
\hline & 87 & 27 & 21.35 & 209127 & First DCPD run \\
\hline & 90 & 28 & 21.35 & 259291 & DCPD \\
\hline & 4 & 29 & 21.35 & 184942 & 10A, $5 \mathrm{~K}$ gain \\
\hline & 3 & 30 & 21.35 & 138407 & Stopped, 10A, 5K gain \\
\hline & 86 & & 21.35 & ------------- & Broke in drilled hole \\
\hline & 77 & 31 & 21.35 & 169554 & No DCPD \\
\hline & 80 & 32 & 21.35 & 244078 & Stopped, 10A, 5K gain \\
\hline & 85 & 33 & 21.35 & 121208 & Stopped, 12A, 5K gain \\
\hline & 76 & 34 & 21.35 & 125869 & Stopped, 12A, 5K gain \\
\hline & 78 & 35 & 21.35 & 167827 & stopped, $12 \mathrm{~A}, 5 \mathrm{~K}$ gain \\
\hline & 84 & & 21.35 & 303277 & Broke in drilled hole \\
\hline & 83 & & 21.35 & & \\
\hline & 43 & 36 & 21.35 & 237246 & Stopped, $12 \mathrm{~A}, 10 \mathrm{~K}$ gain \\
\hline & 44 & 37 & 21.35 & 188271 & Stopped, 12A, 10K gain \\
\hline & 45 & 38 & 21.35 & 211685 & Stopped, 12A, 10K gain \\
\hline & 46 & 39 & 21.35 & 159212 & Stopped, 12A, 10K gain \\
\hline & 47 & 40 & 21.35 & 305965 & Stopped, $12 A, 5 K$ gain \\
\hline
\end{tabular}




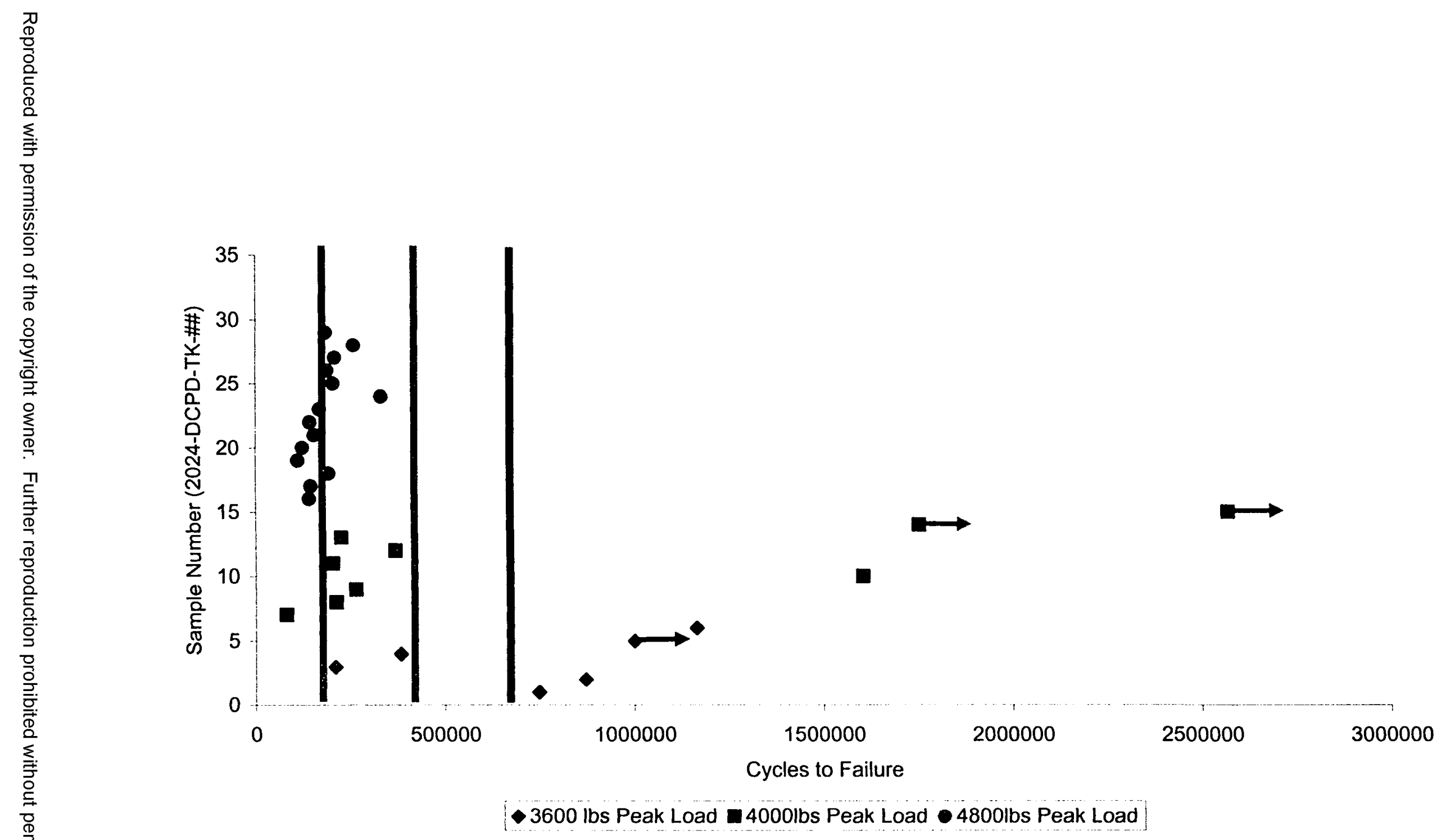

Figure 32. Fatigue results for only the specimens that were tested until failure 
A graphical representation of the data in Table 7 is shown above in Figure 32. The data points with the arrow attached indicate that the test was stopped prior to failure and broken in a tensile test. The solid vertical lines in the figure represent the average fatigue life for each load/stress level used.

\subsection{Surface Replication Technique}

The replicas were examined under optical microscopy and the first crack that was found by the replica technique was over $150 \mu \mathrm{m}$ in length, as shown in Figure 33. The Repliset TI compound was capable of much finer resolutions and detecting short cracks, however, that would require shortening the intervals between replicas and this would make the technique even more time and material consuming. The surface replication technique was already time consuming since a single fatigue test inspected with surface replicas would require approximately five days whereas a fatigue test with EPD or marker bands only required six hours. 


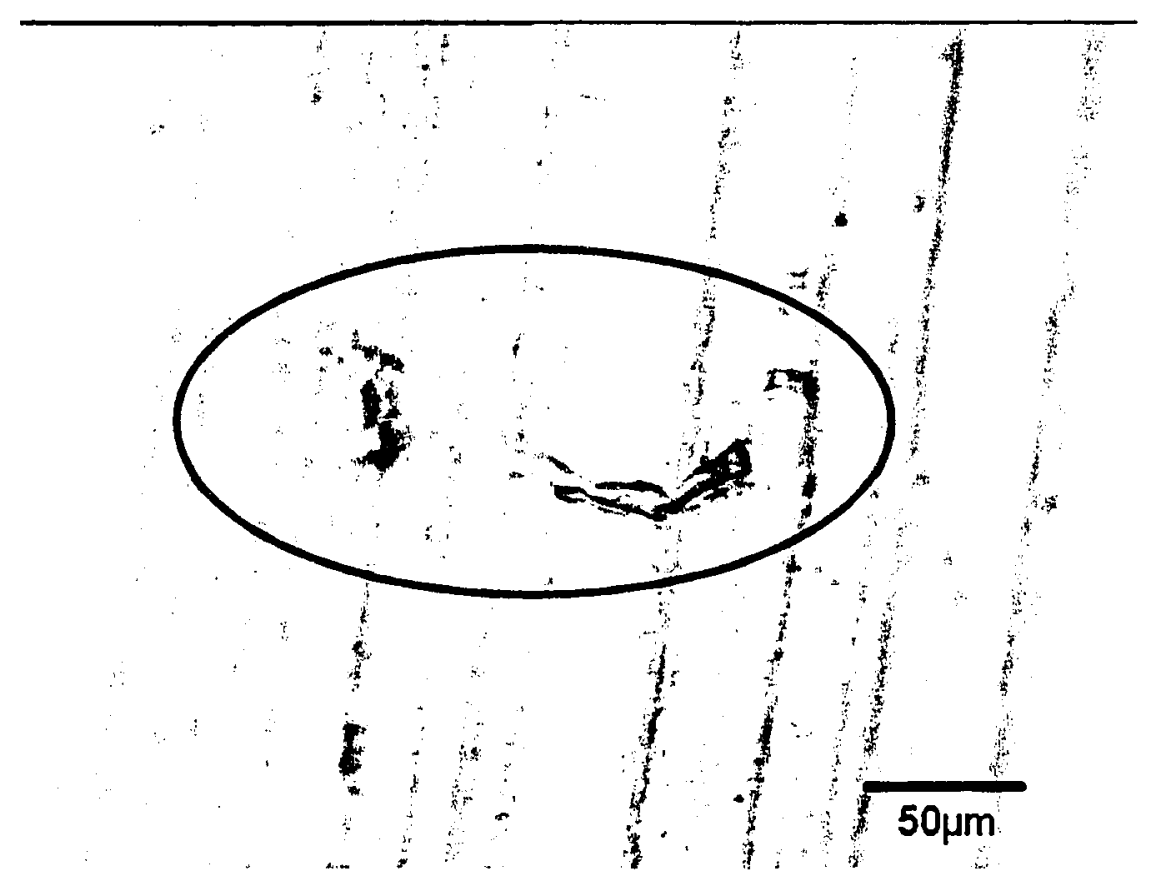

Figure 33. Optical micrograph of smallest crack found with the surface replication technique

In addition, examination of the replicas proved to be difficult due to the shape of the semi-circular notch and the surface finish of the notch surface. The notch shape was difficult to examine because it was difficult to focus on the semi-circular surface.

With the surface replication technique, the only information attainable was the crack characteristics at the surface. Information about the crack front, microstructural controlling features in the short crack regime, crack growth rate, and other such related information could not be obtained from surface replication. This is a significant disadvantage to this technique because it has been shown that in the short crack regime, the surface crack length is not indicative of the crack depth (and hence, the crack size), as the crack front is not uniform [17]. 


\subsection{Marker Bands}

All fatigue loading procedures used in this thesis employed marker bands as described earlier. The marker bands provide a good account for the crack size at a given cycle count, the shape of the crack front, the estimated crack growth rate (approximated by the spacing between the bands) and provide a good calibration method for other detection methods.

During the post-fracture analysis, it was found that the marker bands on the fracture surface were not easily detected using scanning electron microscopy. It was much more effective to use an optical microscope when searching for marker bands on the surface. A typical optical micrograph of a set of marker bands is shown in Figure 34.

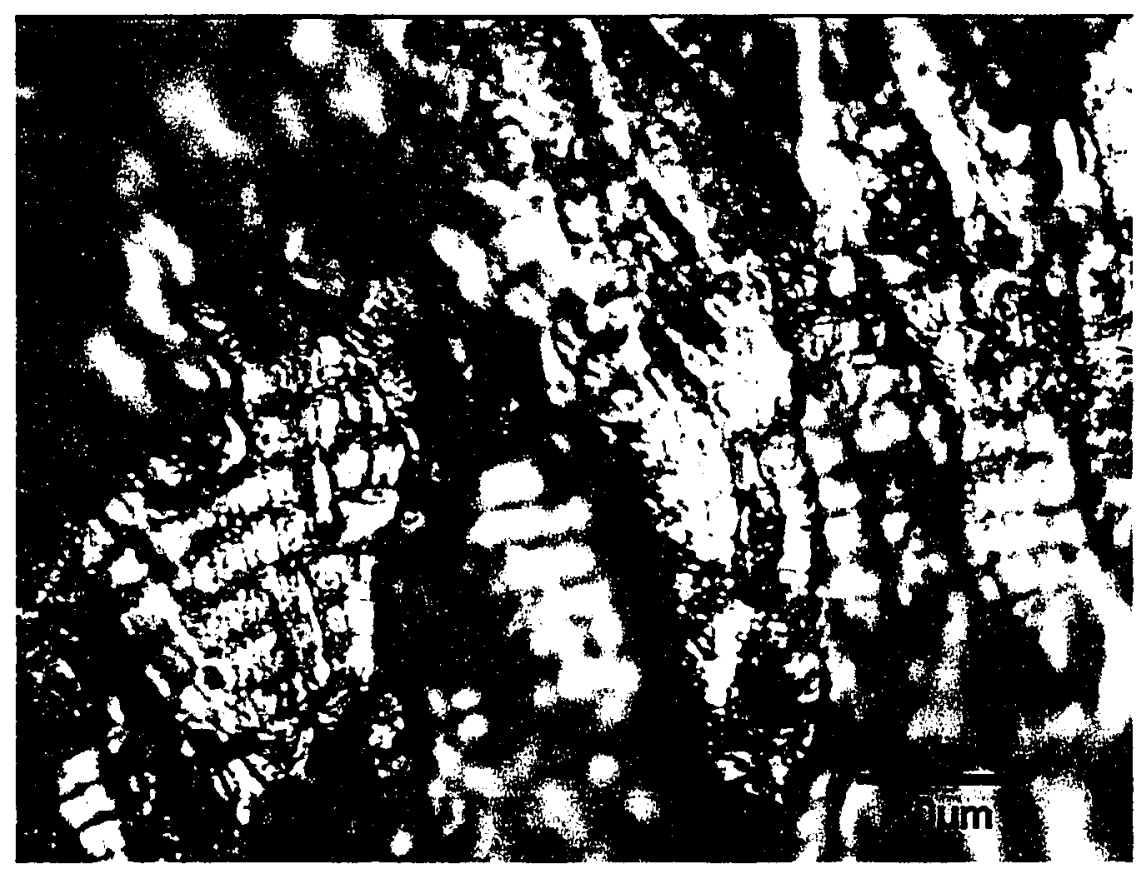

Figure 34. Typical set of marker bands found during optical microscopy 
A typical fracture surface with the identifiable marker bands and their corresponding fatigue cycle count are shown in Figure 35.

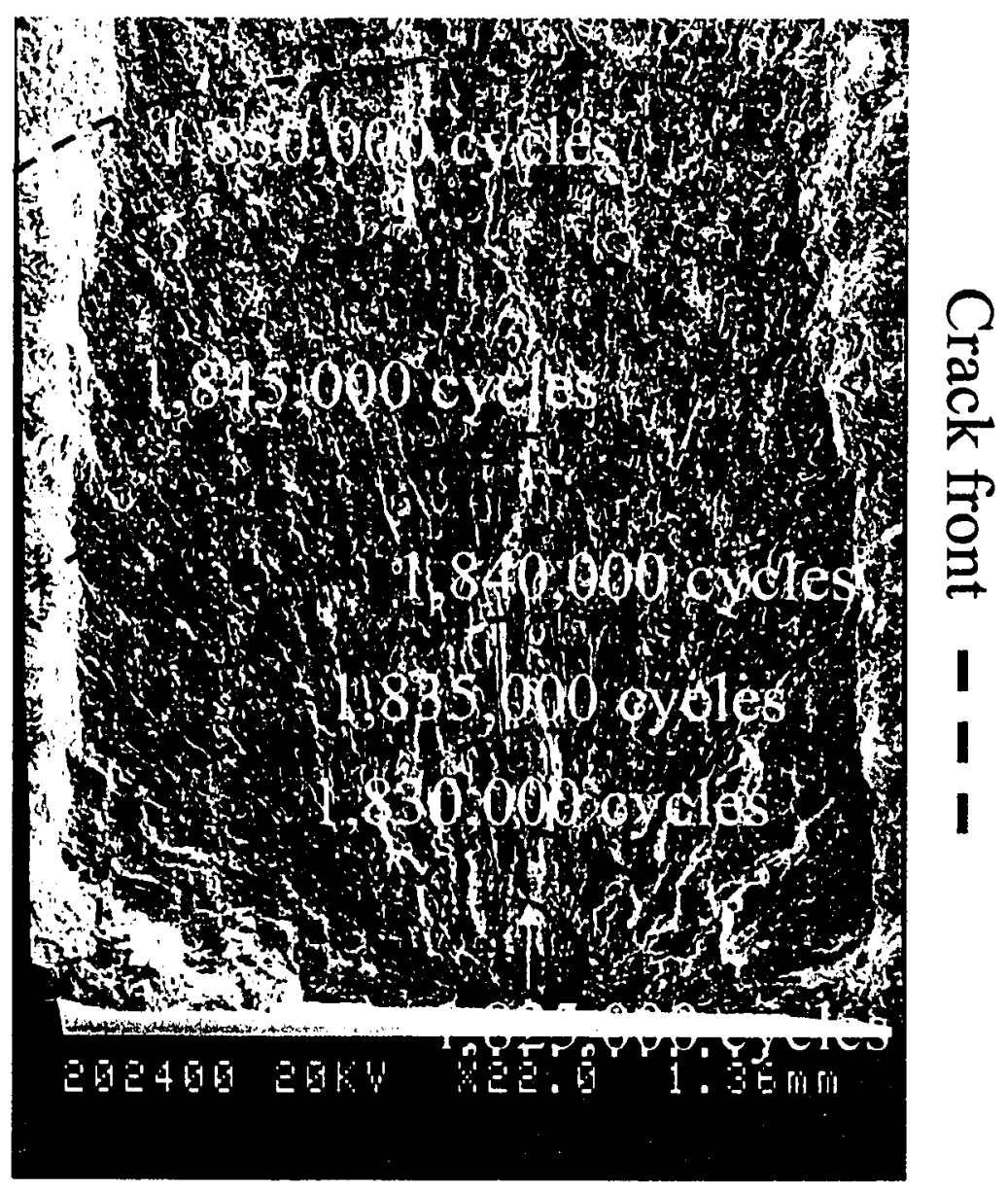

Figure 35. Typical fracture surface with identifiable marker bands shown with the corresponding cycle count.

As can be seen in Figure 35, the identifiable marker bands found on the fracture surfaces were made during the final stages of its fatigue life. In this particular case, the identifiable marker bands were only made during the last 25000 cycles of a sample with a fatigue life of 1850000 cycles, which turns out to be the last $\sim 1.5 \%$ of the fatigue life. It should be mentioned that the sample shown in Figure 35 was a sample that lasted particularly long and the $1.5 \%$ of the fatigue life that is shown by the marker bands is 
relatively low compared to the other fatigue samples. Typically, the fatigue life that is traceable with marker bands is the final $10 \%-15 \%$.

For all of the fatigue tests conducted, no distinguishable marker bands were found in the short crack regime, which is the first two or three grains adjacent to the nucleation site. Even if there were distinguishable marker bands in those first two or three grains, it would be very difficult to gain any useful and significant findings from these marker bands. The findings from the post-fracture analysis, which include many cracks nucleating on the notch wall, cracks growing very rapidly in the short crack regime, and each crack getting arrested in the first couple of grains, show that the crack growth process in the short crack regime is an erratic and non-continuous process. The marker band technique requires continuous crack growth to be most effective because, otherwise, the cycle count could not be aligned with the proper set of marker bands. In other words, if marker bands were found in the short crack regime, it would be unknown when the marker bands were formed because the process was not continuous and they cannot be interpreted by working backwards from the time of fracture as is usually done with marker bands. The only useful information that results from finding a set of marker bands in the short crack regime is the crack front shape and, if the spacing between the marker bands is actually distinguishable, a rough estimate of the crack growth rate.

However, as previously mentioned, because the crack growth rate in the short crack regime is so rapid and the crack becomes arrested at the grain boundaries of the first two or three grains until the stress concentration is great enough for the crack to surpass the barrier and proceed as a long crack, it is theoretically impossible to find a set of marker 
bands in the short crack regime. The time spent for crack propagation in the short crack regime is not long enough to produce a distinguishable set of marker bands before the crack becomes arrested at a grain boundary. Therefore, the marker band technique, while simple to implement, is not an effective method for tracking and studying crack growth properties in the short crack regime.

\subsection{Electrical Potential Drop (EPD)}

It should be noted that all the EPD data in this thesis are voltage drop readings taken at the peak tensile force of the cyclic fatigue testing. This was done to reduce any errors in the measured voltage drop due to shorting errors that occur when crack closure effects allow the crack faces to come in contact even though there are no compressive forces applied in this fatigue testing.

The TK-87 sample was the first successful EPD sample and the potential drop data obtained is shown in Figure 36. The TK-87 sample was run with a current output of $10 \mathrm{~A}$ and a gain setting on the amplifier of 5,000X. 


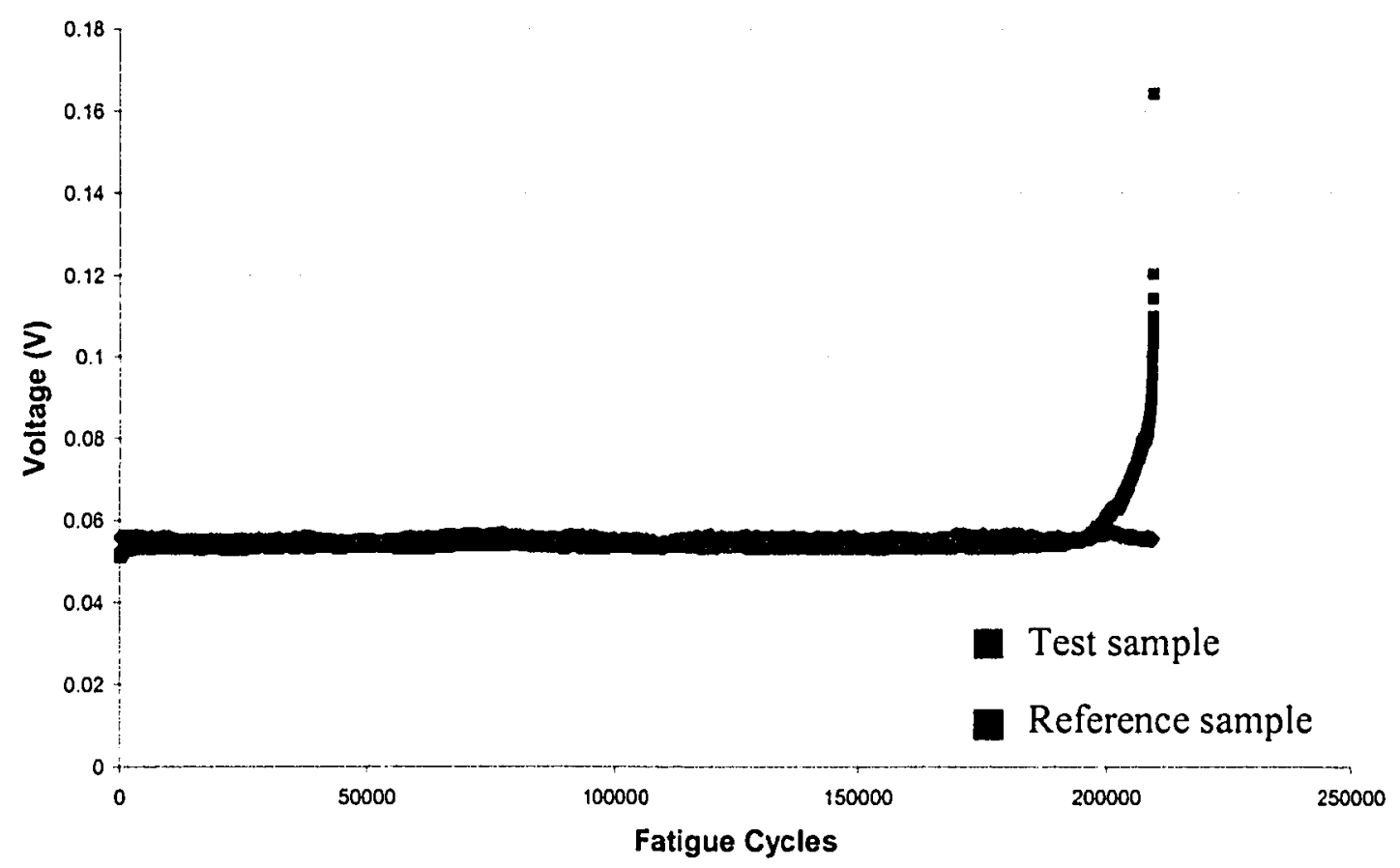

Figure 36. DCPD data for 2024-DCPD-TK-87

Following the procedures outlined above, scanning electron and optical microscopy was used to identify the marker bands on the fracture surface. Figure 37 shows the plot of the output potential drop versus the number of fatigue cycles (same as in Figure 36) but with the crack size superimposed onto it (through the identification of the marker bands). On this sample, seven marker bands were found at crack sizes ranging from $0.37 \mathrm{~mm}$ to 6.1 $\mathrm{mm}$. However, due to the low potential drop output/noise ratio, the smallest detectable crack size in this sample was $1.5 \mathrm{~mm}$. 


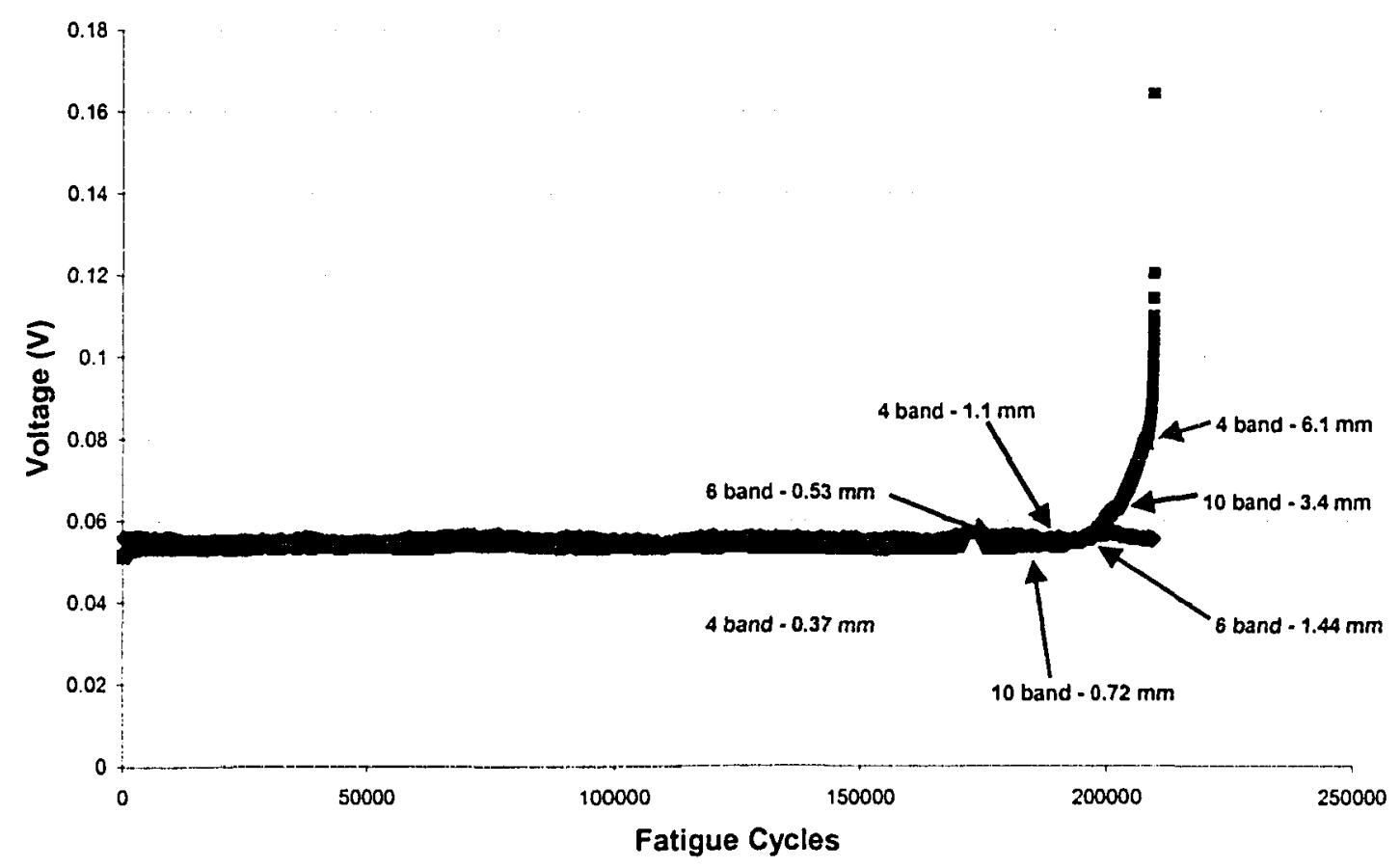

Figure 37. DCPD and marker band data for 2024-DCPD-TK-87

A detectable crack size of $1.5 \mathrm{~mm}$ is too large if, as theorized, the short crack regime is only about the size of three grains. The crack plane for the samples tested in this study was the ST plane and the cracks radiate semi-circularly from the nucleation point. The grain size in the transverse and short transverse direction is approximately $116 \mu \mathrm{m}$ and 33 $\mu \mathrm{m}$, respectively, thereby indicating an estimated short crack region of between $100 \mu \mathrm{m}$ and $350 \mu \mathrm{m}$. Therefore, for the EPD detection method to be a viable method for detecting and monitoring short crack propagation, it must be able to detect cracks less than $350 \mu \mathrm{m}(0.35 \mathrm{~mm})$ in size.

After running a series of five tests using identical settings (10 A and $5000 \mathrm{X}$ gain) and confirming that these settings did not provide a sufficient crack resolution for this short crack study, the settings for the EPD testing were altered to increase the crack resolution. 
The reason the crack resolution was not sufficient is because the signal-to-noise ratio was too low and any small changes in the measured electrical potential from a small crack nucleating and propagating are masked by the signal's electrical noise.

As mentioned earlier, a couple of different methods were used to increase the signal-tonoise ratio. Firstly, the applied current from the power supply was increased from $10 \mathrm{~A}$ to $12 \mathrm{~A}$ while maintaining the same gain setting of $5,000 \mathrm{X}$. Increasing the applied current from the power source will provide a higher initial voltage reading and increase the signal-to-noise ratio because the voltage noise variation will remain similar to the variation seen at the lower current level of $10 \mathrm{~A}$. As mentioned in the procedure section, the highest current that could be used in the EPD testing was $12 \mathrm{~A}$ because it was found that prolonged application of any voltage higher than $12 \mathrm{~A}$ would cause the sample to heat up significantly above room temperature and because a relay that could handle that much current could not be found or made in the allotted time, a constant current flow was the only option.

A total of 11 tests were run using these settings and while the crack resolution showed slight improvement from the increase in the voltage signal and signal-to-noise ratio, the crack resolution was still too low. The best result obtained from these settings is shown in Figure 38 and Figure 39. Figure 38 shows the averaged (every 1000 data points) EPD data obtained from this result and based on the electrical voltage noise, the most sensitive the limits that could be set was for a $10 \mathrm{mV}$ change. Immediately after the voltage saw an increase of $10 \mathrm{mV}$, the fatigue testing was stopped and the sample was immediately overloaded and broken in tension. Figure 39 is the SEM micrograph of this specimen's 
fracture surface and the crack front is outlined by the red, dashed line. As can be seen in this figure, the crack front has a semi-elliptical shape and has a radius of about $1.3 \mathrm{~mm}$, which, while showing an improvement of $0.2 \mathrm{~mm}(200 \mu \mathrm{m})$, is still too large.

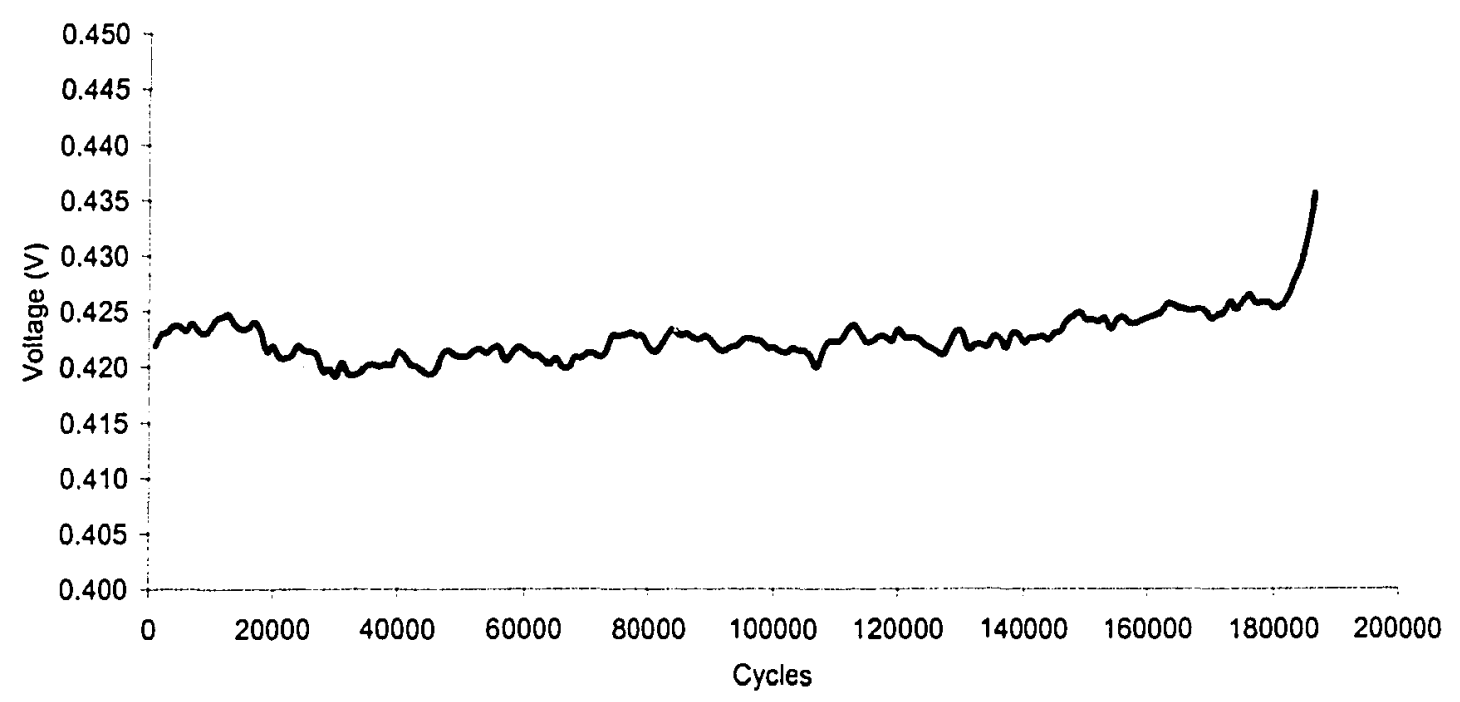

Figure 38. Typical EPD data plot for a sample tested with 12A and 5000X gain (2024-DCPD-TK-52) 


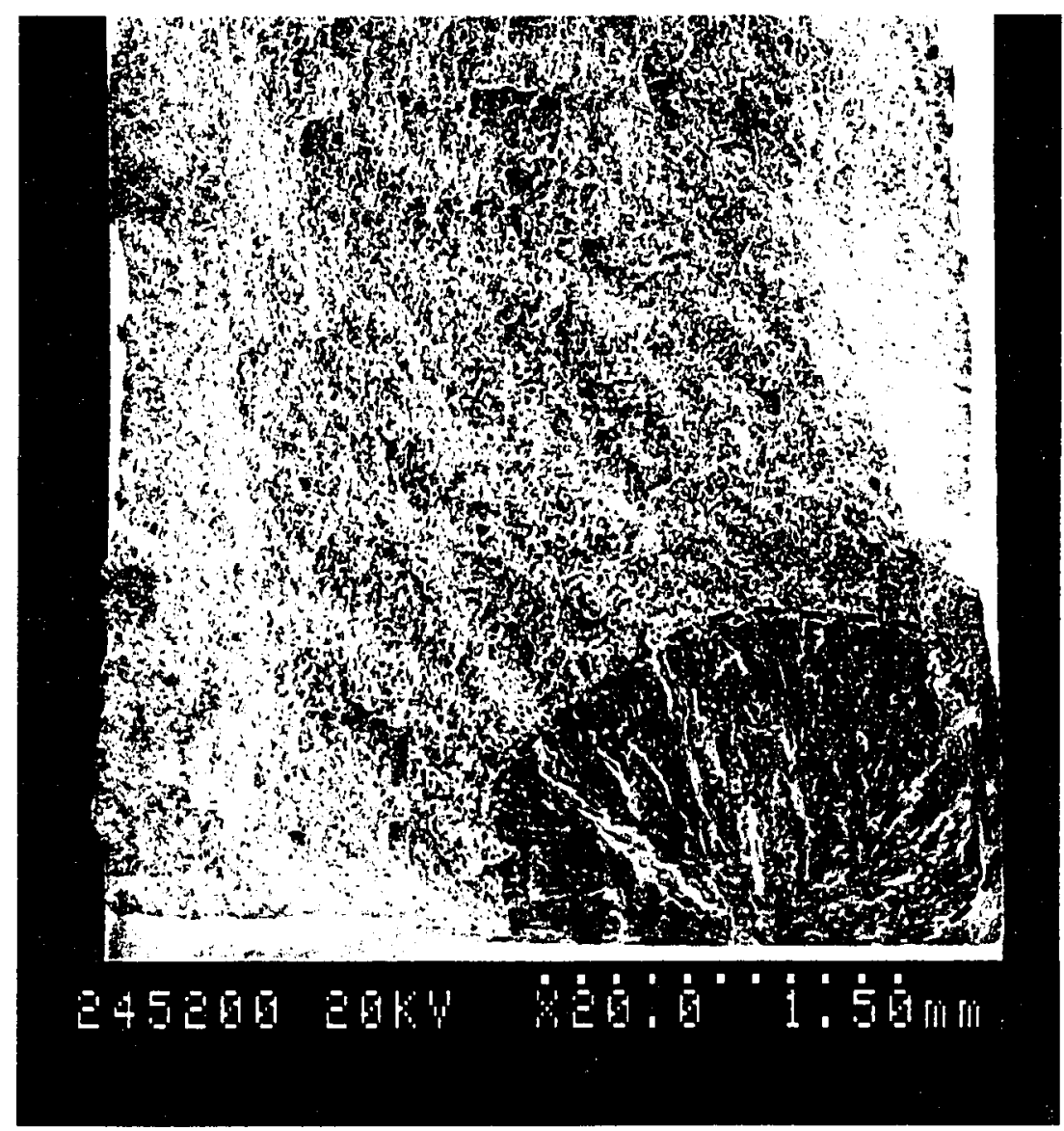

Figure 39. This is a SEM micrograph of the above sample showing the $1.3 \mathrm{~mm}$ crack detected by EPD method.

The second method of improving the crack resolution in the EPD testing was to increase the gain of the amplifiers in order to increase the initial voltage signal and hopefully, the signal-to-noise ratio as well. The limitation and disadvantage of increasing the gain setting of the amplifier is that while the voltage signal will increase, the electric noise of the EPD system will also increase. Therefore, while it is desirable to have a high gain setting to have a high voltage signal and high signal-to-noise ratio, the gain setting must not be too high or else the increased electrical noise will negate any gains from the increased voltage signal. 
Initially, the gain setting was increased from $5,000 \mathrm{X}$ to $10,000 \mathrm{X}$ and a total of nine fatigue EPD specimens were run at this setting. However, it was immediately noticeable that the voltage readings were not very stable and may cause problems during fatigue testing. As a result, a voltage drop stability test was run using one of the test samples under no applied load. The result from this stability test is shown in Figure 40. As can be seen, the variation is greater than what was seen in the previous stability tests where the gain of the amplifier was only 5,000X. After the system has been warmed up $(\sim 5,000$ seconds), the voltage drop readings still fluctuate by as much as $20 \mathrm{mV}$, which while significantly increased, is expected because the fluctuations seen in the previous tests with a 5,000X gain saw fluctuations below $10 \mathrm{mV}$ and now that the amplifier gain has been doubled to $10,000 \mathrm{X}$, it is expected that the electrical noise would also double.

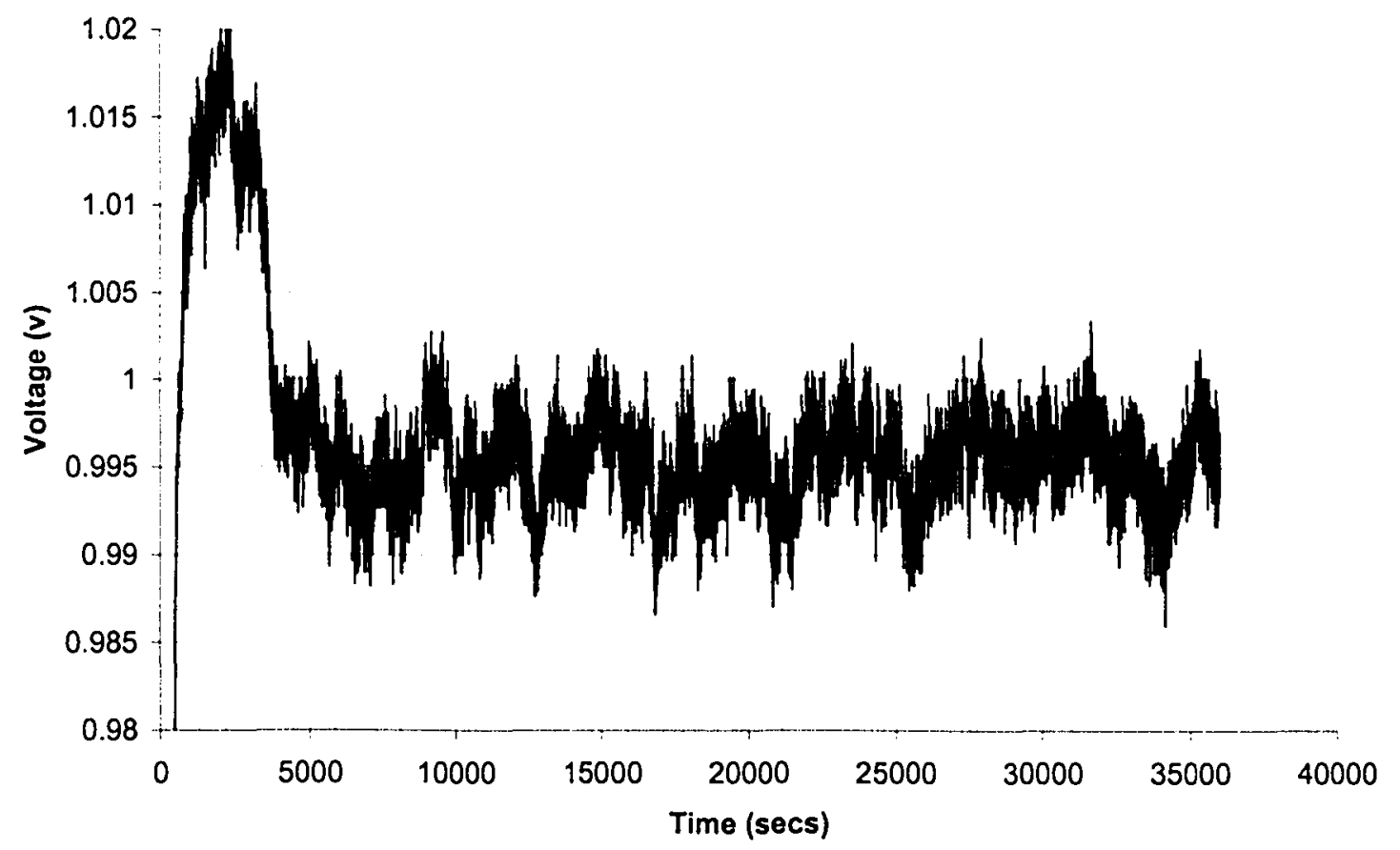

Figure 40. Static voltage stability test using $12 \mathrm{~A}$ of current and $10000 \mathrm{X}$ gain 
However, the problem of increased electrical noise was secondary to the stability of the voltage drop signal. This did not occur during the static voltage stability test but came very apparent and problematic during the actual EPD fatigue testing. It was found that the voltage signal dramatically increased or decreased during the fatigue testing for no apparent reason, in some cases, by as much as $80 \mathrm{mV}$. This phenomenon occurred in most of the samples tested and typical examples are shown in Figure 41 and Figure 42. As can be seen, the voltage drop signal is erratic and even after extensive data averaging, all of the test data in that EPD test is completely worthless.

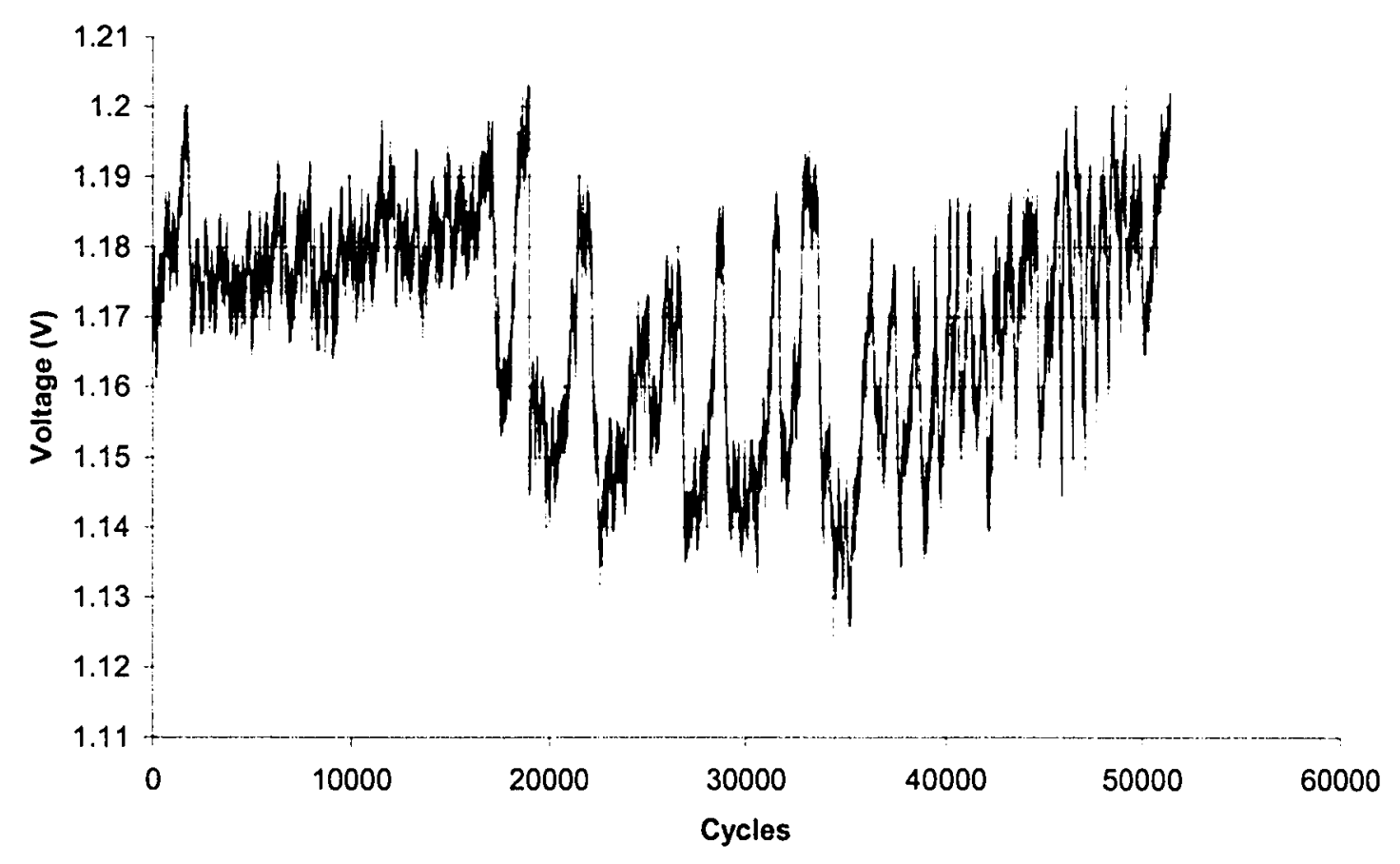

Figure 41. EPD voltage drop data for sample TK-59 


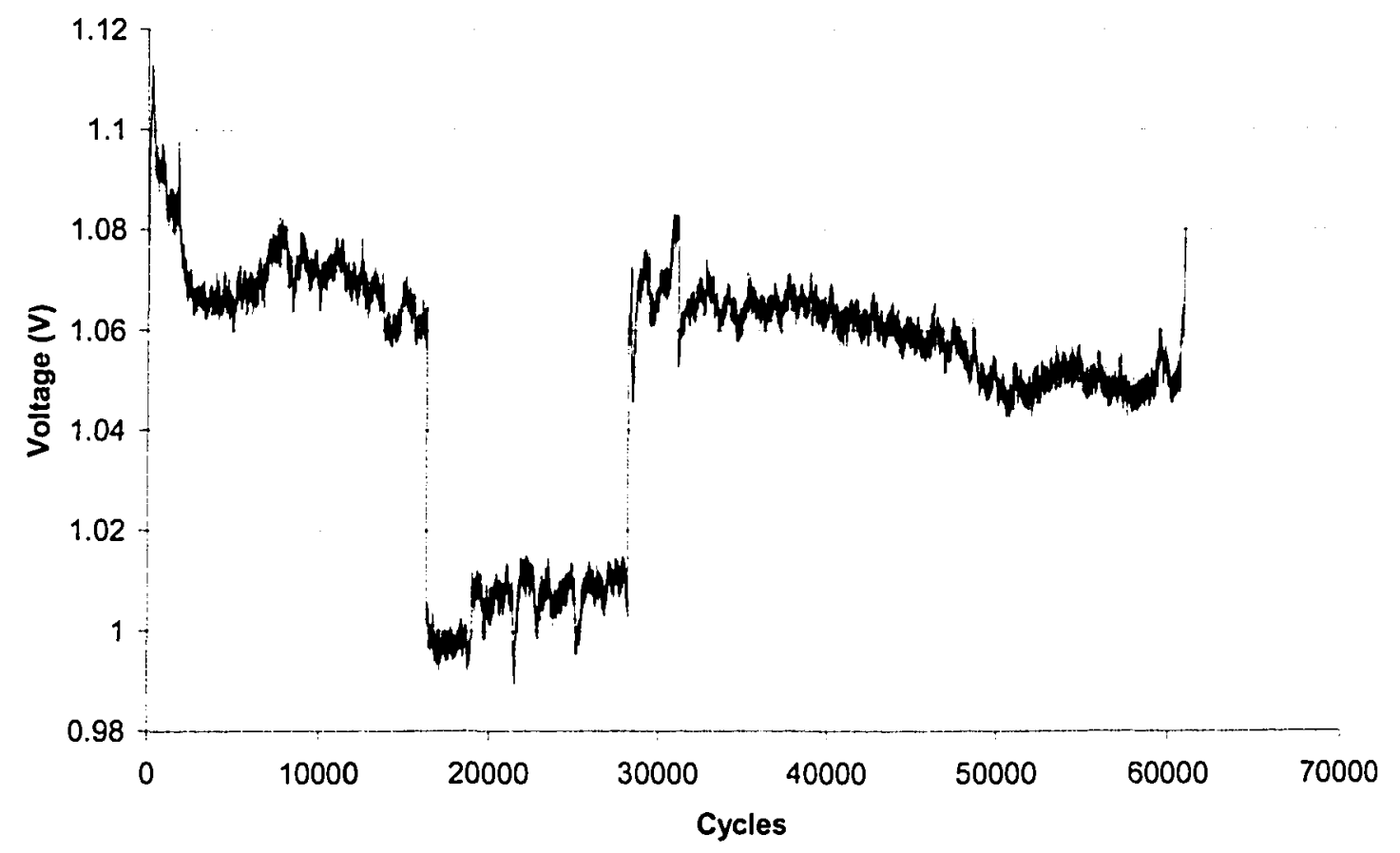

Figure 42. EPD voltage drop data for sample TK-60

During every test where such an erratic jump/drop in electrical potential was seen, the fatigue test was stopped and the sample was examined visually using a traveling microscope and on every occasion, no cracks were found. In addition, all of the wiring and equipment were examined and no loose connections were found.

After many attempts at the settings described above, one of the samples returned a promising result. The EPD data for this sample showed no erratic behaviour and the electrical noise variation was only about $10 \mathrm{mV}$ as shown in Figure 43 . The fracture surface, shown in Figure 44, shows the $250 \mu \mathrm{m}$ radii crack detected by the EPD system using the aforementioned settings. This is a significant improvement over the $1.3 \mathrm{~mm}$ $(1300 \mu \mathrm{m})$ detection limit seen with a lower amplification setting and is within the short crack regime. This result was seen by a change of $10 \mathrm{mV}$ in the potential drop signal and 
this is the greatest detection capability using these settings because the erratic jumps in the voltage signal hiding any voltage changes less than $10 \mathrm{mV}$. In most cases, a $10 \mathrm{mV}$ voltage change is not even noticeable and undetectable with the unstable voltage readings.

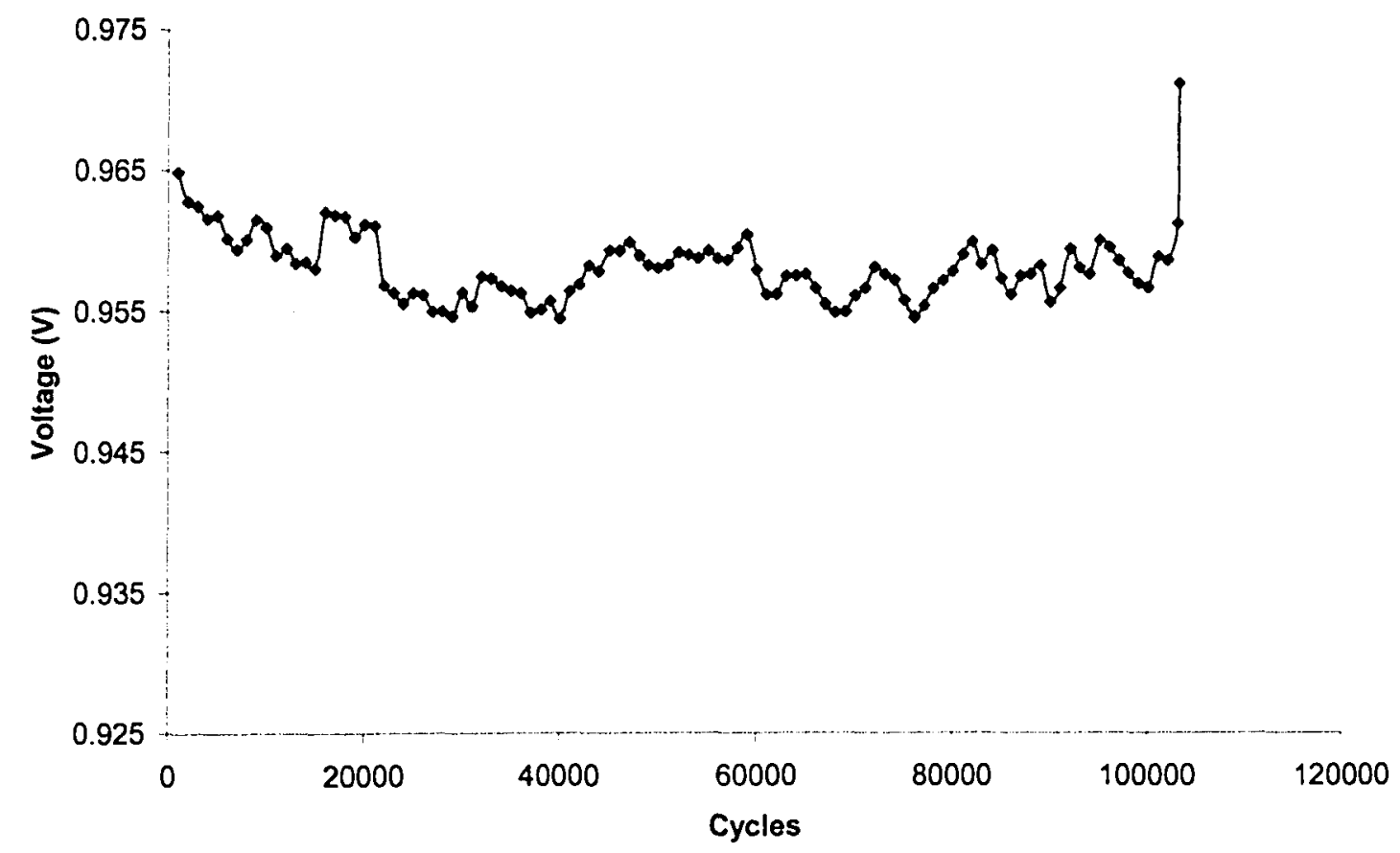

Figure 43. Averaged EPD data from sample TK-57 


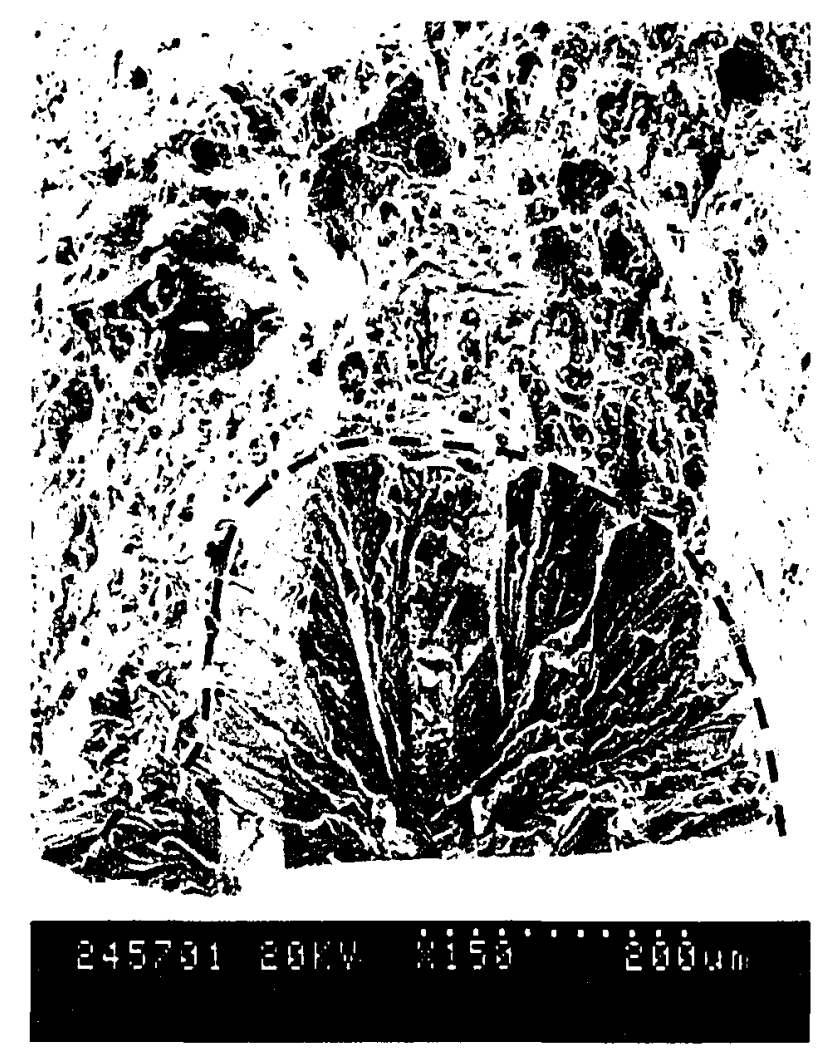

Figure 44. Fracture surface of sample TK-57 showing $250 \mu \mathrm{m}$ radii crack found during fatigue testing

Because the dramatic increase in gain of the amplifier seemed to have caused the erratic behaviour of the voltage readings, it was decided to lower the gain while still maintaining a high voltage signal (for sufficient crack resolution) and to reduce the amount of scatter observed in the voltage readings. It was speculated that since the maximum gain setting on the amplifiers was $11,000 \mathrm{X}$ that using a gain setting of $10,000 \mathrm{X}$ was near the capability limit of the amplifier and that the erratic and unstable behaviour of the voltage drop signal could be a result of equipment instability in addition to the amplification of the existing signal noise. As a result, the gain setting of the amplifier was set to $8,000 \mathrm{X}$ while maintaining all other previous EPD settings. 
Using the new gain setting, 14 EPD fatigue tests were run and it was immediately noticeable that the voltage signal was a lot more stable. A detection limit of $10 \mathrm{mV}$ was now very feasible, reliably detectable and relatively trouble-free. In the previous set of tests run at 10,000X gain, the test operator had to continuously alter the upper voltage detection limit as the test was running and speculate as to when a slight voltage increase of $10-20 \mathrm{mV}$ was a result of the presence of a short crack or whether it was noise in the EPD system. Therefore, in almost all of the cases with the 10,000X gain setting, short cracks were missed because the small voltage drop increase from the presence of a small crack was hidden within the erratic signal and misinterpreted as just signal noise. Theoretically, the 10,000X gain setting should allow improved crack resolution over the lesser gain setting, however, in practice, this was not the case as the lesser $8,000 \mathrm{X}$ gain setting provided a much more stable and usable signal that ultimately led to a superior crack resolution, given the equipment available. This is clearly evident by the $42 \mu \mathrm{m}$ radii crack found in sample TK-65 shown in Figure 45 and Figure 46. Figure 45 shows the averaged EPD voltage increase $15 \mathrm{mV}$ during the fatigue test. This increase of 15 $\mathrm{mV}$ translated to a crack size that had an estimated radius of $42 \mu \mathrm{m}$, as shown in Figure 46, and when compared to optical microscopy micrographs taken during the metallographic examination, this crack closely resembles many of the grains observed. The test was stopped after a $15 \mathrm{mV}$ increase because the raw voltage data was too erratic to distinguish a voltage change less than $15 \mathrm{mV}$. 


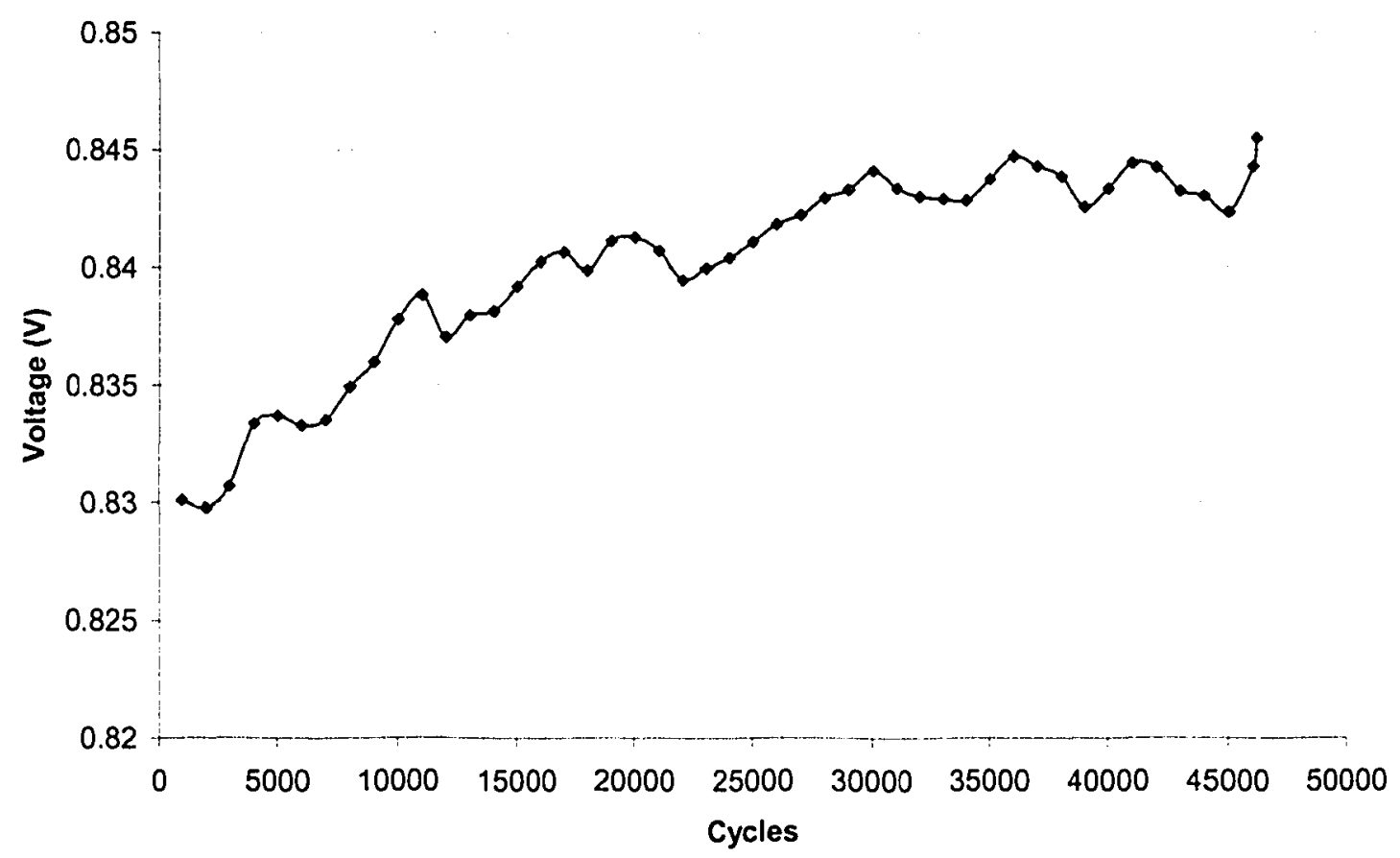

Figure 45. Averaged EPD data from sample TK-65

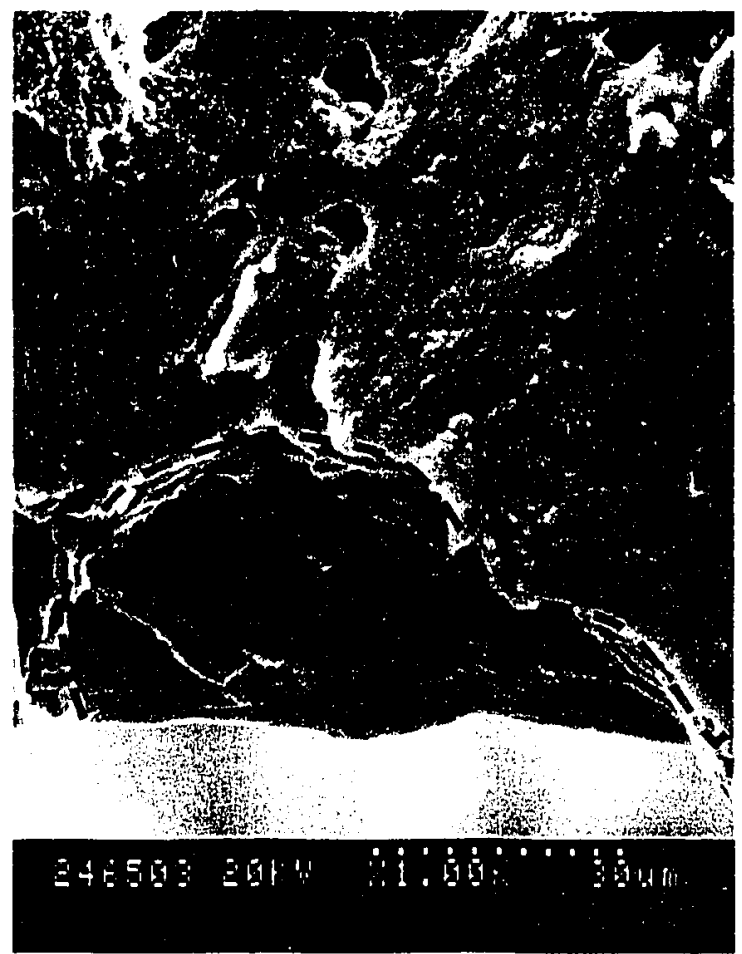

a)

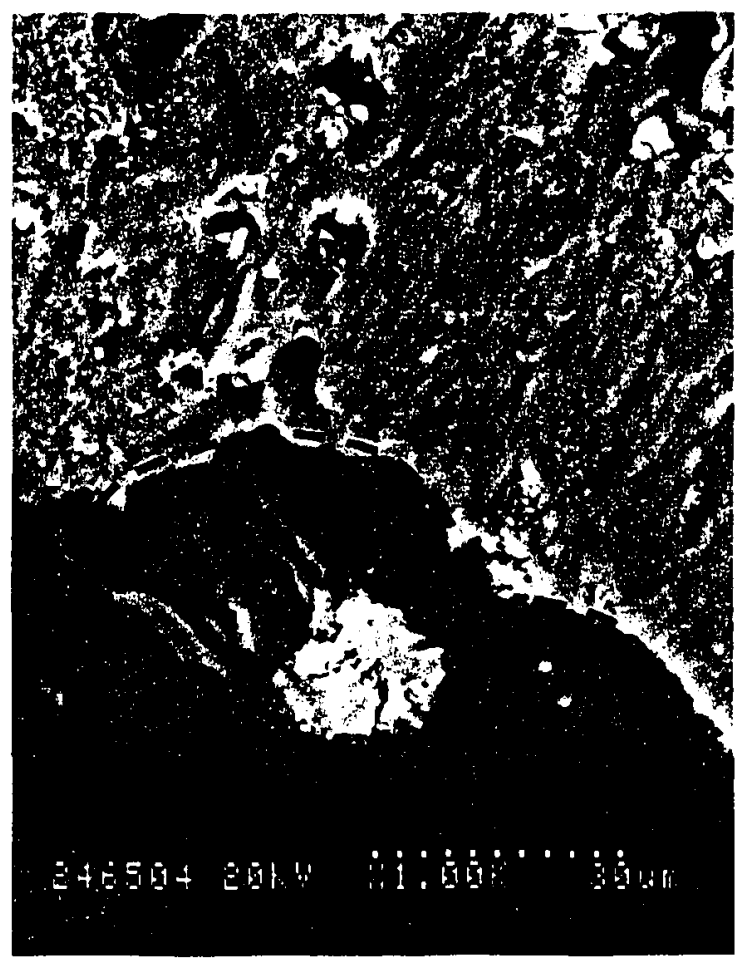

b)

Figure 46. Fracture surface of sample TK- 65 showing a) $42 \mu \mathrm{m}$ radii crack found during fatigue testing and b) the same crack using the backscattered electron detector to show the size of the constituent particle in relation to the crack size. 
It should be noted that almost all of the EPD data presented has gone through significant data averaging to reduce scattered data and to clearly show trends in the data charts. The raw data received from the EPD process is more erratic and scattered than the EPD graphs lead to believe. Much of this has been attributed to the aging equipment that was used during the EPD testing and the lack of a fatigue crack growth rate analysis software.

Another possible source of error that could help produce erratic voltage drop readings is that when the EPD method uses a DC power supply, it becomes susceptible to thermoelectric effects and produce DC potentials on top of those which are produced by the specimen's electrical field. These problems are usually corrected by reversing the direction of current, however, due to equipment limitations, this was not possible for this project. There were also errors associated with voltage lead wire placements. The electrical field in the region near the crack tip is highly non-uniform and therefore, even small variations in the voltage lead wire placement can cause significant changes in the measured electrical potential drop for the same crack size. Lastly, there may have been some interference with induced EMF because the voltage measurement lead wires were not twisted to reduce stray voltages induced by changing magnetic fields.

However, even with out-of-date testing equipment, no fatigue crack growth software and other external sources of error, it was still evident from the EPD testing performed in this study that the EPD method can be used with success in detecting short fatigue cracks in 2024-T3, and presumably in other high strength aluminum alloys used in aerospace applications such as AA7075 and AA7050, which exhibit similar electrical properties. 


\subsection{Post-Fracture Analysis}

After the fatigue tests were completed, all of the fracture surfaces were examined by scanning electron microscopy (SEM) to study the crack nucleation site and the surrounding area (short crack regime). In almost every case, the fatigue crack(s) nucleated from a constituent particle. An example of typical crack nucleation sites using the backscattered electron detector is shown in Figure 47.

Crack tunneling (otherwise known as crack curvature) was present in most of the fatigue specimens. Typically, when crack tunneling is present, visual inspection methods may not suffice because the actual crack length may be greater than the surface crack. Typically in these situations, ASTM requires that if a contour is visible, the fracture surface be examined in at least two locations in order to determine the through-thickness crack curvature. It would also be recommended that a three-point, average throughthickness crack size be calculated and be compared with the crack size measurement. The difference between the two crack sizes will be the crack curvature correction.

In addition, all of the SEM micrographs taken of the crack nucleation sites were examined in the image analysis software similar to that performed during the microstructural analysis. All of the data for these constituent particles are compiled in Table 8. 


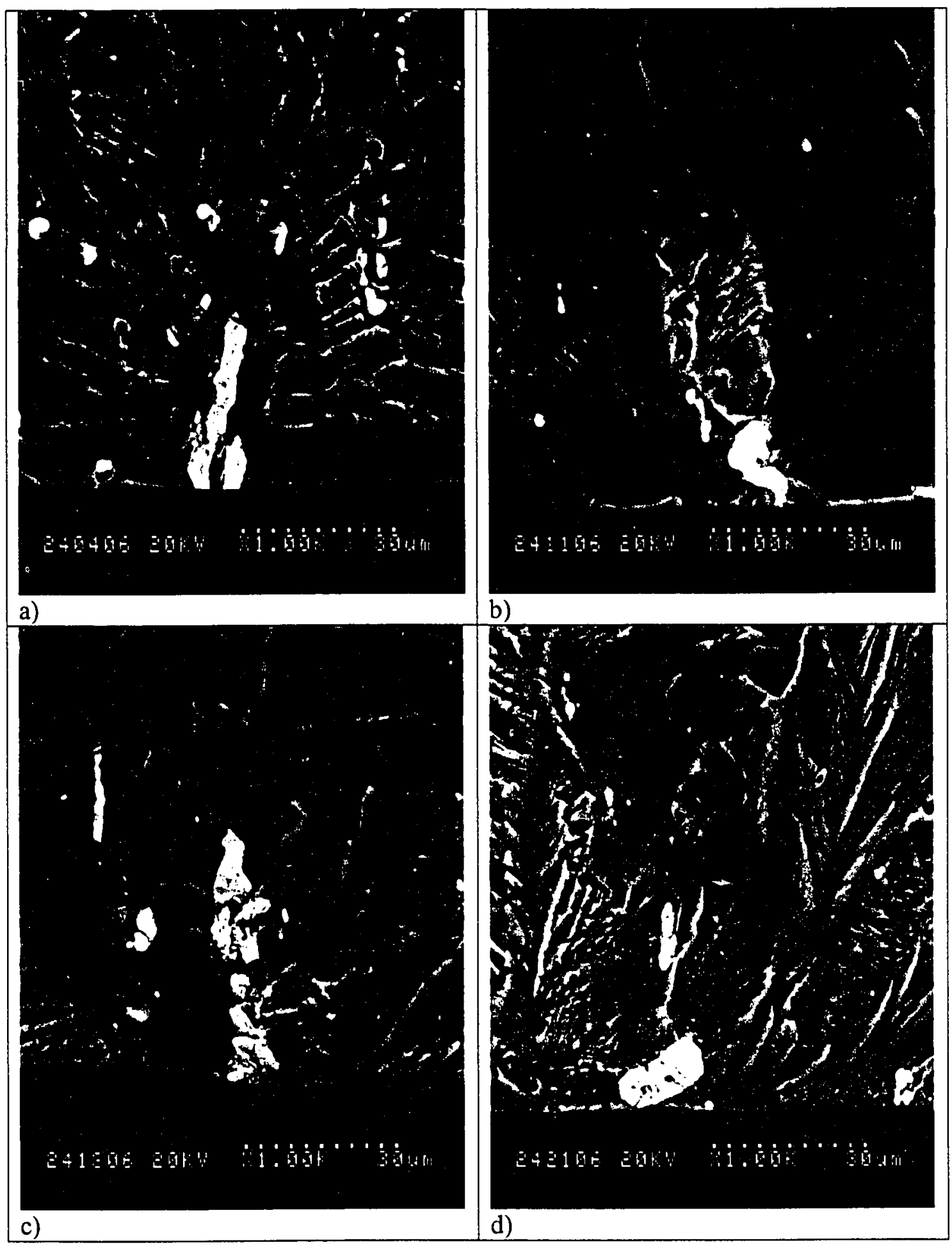




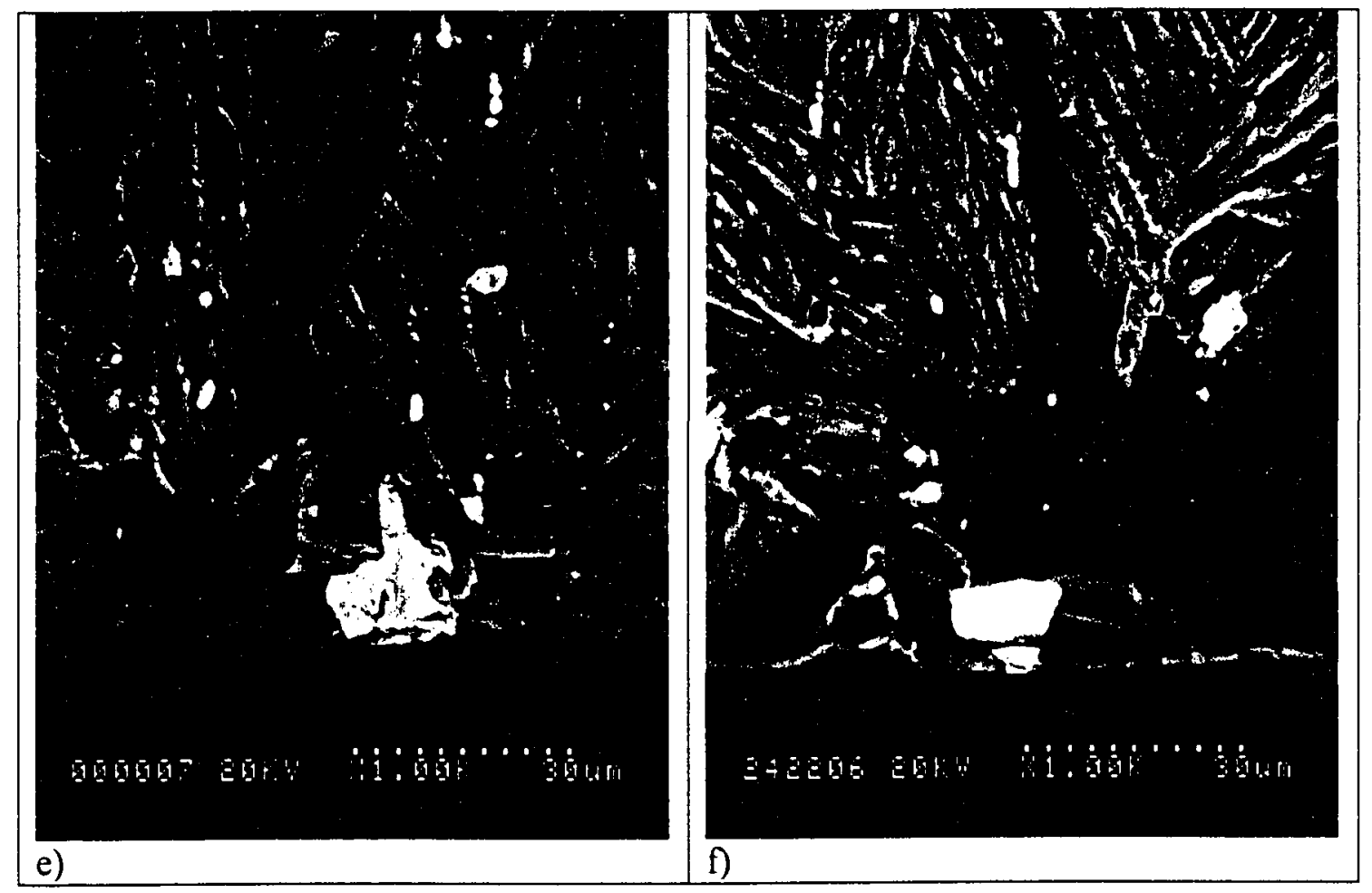

Figure 47. Typical crack nucleation sites found using the backscattered electron detector in the SEM 
Table 8. Constituent particle data collected from the crack nucleation region

\begin{tabular}{|c|c|c|c|c|}
\hline Sample & Area $\left(\mu \mathrm{m}^{2}\right)$ & $\begin{array}{c}\text { Aspect } \\
\text { Ratio }\end{array}$ & $\begin{array}{c}\text { Size - Length } \\
(\mu \mathrm{m})\end{array}$ & $\begin{array}{c}\text { Size - Width } \\
(\mu \mathrm{m})\end{array}$ \\
\hline 3 & 162.55283 & 2.318305 & 26.167801 & 13.265564 \\
\hline 4 & 172.76898 & 3.8782111 & 33.698441 & 11.551743 \\
\hline 5 & 93.370361 & 7.4489663 & 27.868385 & 4.4143829 \\
\hline 6 & 126.22871 & 2.5604894 & 23.764139 & 12.129082 \\
\hline 8 & 302.91028 & 2.2763295 & 35.217594 & 17.314987 \\
\hline 10 & 102.95168 & 1.2886504 & 13.371174 & 11.526358 \\
\hline 11 (a) & 80.968483 & 1.9951364 & 16.988024 & 9.7301559 \\
\hline $11(b)$ & 127.60535 & 1.8695732 & 16.74781 & 10.466969 \\
\hline $11(c)$ & 218.93491 & 2.7611907 & 31.788574 & 13.545938 \\
\hline $11(e)$ & 65.161209 & 1.5094873 & 12.339031 & 9.0861282 \\
\hline 13 & 383.87875 & 5.0127828 & 49.463615 & 13.312439 \\
\hline $14(a)$ & 282.2485 & 1.4662577 & 22.528107 & 17.241856 \\
\hline $14(b)$ & 64.229393 & 1.6706519 & 11.44577 & 7.6955872 \\
\hline $14(c)$ & 70.897232 & 3.7635883 & 20.14617 & 6.460022 \\
\hline 15 & 178.26349 & 1.0971898 & 16.985428 & 16.866539 \\
\hline 17 & 142.25334 & 2.070268 & 18.249519 & 9.4927387 \\
\hline 18 & 217.18391 & 1.5634184 & 23.185612 & 15.802368 \\
\hline $20(a)$ & 255.9715 & 1.1496082 & 21.976227 & 19.433599 \\
\hline $20(b)$ & 329.04239 & 1.2943829 & 25.501751 & 22.69342 \\
\hline 21 & 72.55162 & 1.6737597 & 14.915436 & 8.4819784 \\
\hline 22 & 132.95496 & 1.1745181 & 14.729084 & 13.449415 \\
\hline 27 & \multicolumn{4}{|c|}{ no crack found } \\
\hline 28 & 182.26059 & 6.7659073 & 38.268768 & 6.4572144 \\
\hline 30 & 241.29935 & 1.530647 & 24.540848 & 17.094736 \\
\hline 32 (a) & 74.218086 & 1.4975305 & 12.549736 & 11.125608 \\
\hline $32(\mathrm{~b})$ & 217.11145 & 3.3190837 & 33.402016 & 10.910568 \\
\hline $33(a)$ & 208.18741 & 1.1573863 & 19.257166 & 19.17194 \\
\hline $33(\mathrm{~b})$ & 288.75739 & 1.9326273 & 29.982538 & 14.336404 \\
\hline $34(a)$ & 411.10974 & 1.439532 & 28.034485 & 19.302969 \\
\hline $34(\mathrm{~b})$ & 238.30455 & 1.3677421 & 22.464979 & 16.072952 \\
\hline $34(c)$ & 111.85847 & 1.3189285 & 18.617655 & 15.500175 \\
\hline 34 (d) & 49.655838 & 1.9132145 & 13.708868 & 7.562561 \\
\hline $35(a)$ & 100.73663 & 2.2151927 & 19.010223 & 11.509743 \\
\hline $35(\mathrm{~b})$ & 207.8372 & 1.9071445 & 23.858093 & 14.476379 \\
\hline $36(a)$ & 184.34972 & 3.3671934 & 30.210899 & 14.482025 \\
\hline $36(\mathrm{~b})$ & \multicolumn{4}{|c|}{ no particle } \\
\hline $36(c)$ & 197.9471 & 1.2364203 & 18.909294 & 14.506256 \\
\hline 37 & 123.65656 & 3.3238547 & 22.253021 & 7.381073 \\
\hline $38(a)$ & 183.49232 & 1.6743621 & 19.393597 & 13.035056 \\
\hline $38(\mathrm{~b})$ & 152.85593 & 1.4434196 & 19.082367 & 14.980637 \\
\hline $39(a)$ & 262.80643 & 1.6273375 & 23.487377 & 15.868521 \\
\hline $39(\mathrm{~b})$ & 67.636757 & 4.3752565 & 19.671188 & 6.4526062 \\
\hline 40 (a) & \multicolumn{4}{|c|}{ no particle } \\
\hline $40(\mathrm{~b})$ & 139.35515 & 1.9327604 & 22.617828 & 13.079422 \\
\hline 41 & 128.31783 & 1.2545697 & 21.035324 & 15.040726 \\
\hline $43(a)$ & 134.66972 & 1.1921979 & 17.387102 & 15.816154 \\
\hline
\end{tabular}




\begin{tabular}{|c|c|c|c|c|}
\hline $43(b)$ & \multicolumn{4}{|c|}{ no particle } \\
\hline $44(a)$ & 288.15359 & 2.2544989 & 29.305294 & 14.685913 \\
\hline $44(b)$ & 52.590263 & 2.2445466 & 12.473511 & 6.8845673 \\
\hline 44 (c) & 230.45447 & 1.0795036 & 18.412891 & 16.530476 \\
\hline 45 & 371.89951 & 2.7532864 & 37.449677 & 15.245277 \\
\hline 46 (a) & 281.92245 & 1.2581024 & 28.046993 & 21.79845 \\
\hline $46(\mathrm{~b})$ & 214.491 & 2.4173747 & 24.175812 & 14.75703 \\
\hline 47 & 98.345604 & 1.2448387 & 13.718487 & 11.391022 \\
\hline 48 & 357.45682 & 1.8172697 & 27.291656 & 18.018221 \\
\hline 49 & 233.70364 & 1.4243099 & 23.563812 & 15.745161 \\
\hline 50 & \multicolumn{4}{|c|}{ no particle } \\
\hline 51 & 393.58771 & 1.0510176 & 24.059654 & 23.582886 \\
\hline 52 & 144.78928 & 2.5344191 & 19.625992 & 9.4471722 \\
\hline 53 & 203.28462 & 1.9997336 & 24.020868 & 13.610458 \\
\hline 56 & 365.48724 & 1.6263261 & 28.593452 & 16.947823 \\
\hline 57 & 168.69943 & 1.4283988 & 19.235159 & 15.600674 \\
\hline 58 & 553.52008 & 1.9343414 & 39.562378 & 25.219971 \\
\hline 61 & 210.30067 & 1.6222799 & 23.668488 & 14.493407 \\
\hline 62 & 179.76089 & 3.1319859 & 27.96666 & 12.306885 \\
\hline 63 & 270.63156 & 1.3851518 & 21.155437 & 15.849455 \\
\hline 64 & 289.02304 & 3.9238825 & 36.383347 & 10.279633 \\
\hline 65 & 268.5545 & 1.1534175 & 19.767021 & 17.516556 \\
\hline 66 & 197.91087 & 1.3525528 & 19.471985 & 17.918886 \\
\hline 67 (a) & 356.09225 & 2.4164674 & 31.611542 & 16.790428 \\
\hline 67 (b) & 117.05108 & 1.9848171 & 18.136181 & 10.548584 \\
\hline $68(a)$ & 51.310226 & 1.2385964 & 8.6161423 & 6.8706131 \\
\hline $68(b)$ & 101.05059 & 1.4376973 & 14.251989 & 9.9099083 \\
\hline 69 (a) & 132.01303 & 1.1359206 & 20.332275 & 16.578388 \\
\hline 69 (b) & 88.793617 & 2.0981632 & 15.193787 & 7.5974445 \\
\hline $69(c)$ & 79.072571 & 2.083996 & 14.748302 & 7.235436 \\
\hline 70 & 288.0932 & 2.6209966 & 30.766235 & 12.70661 \\
\hline 71 & \multicolumn{4}{|c|}{ no crack found } \\
\hline 72 & \multicolumn{4}{|c|}{ no crack found } \\
\hline 73 & 145.9727 & 5.0342537 & 35.548462 & 10.127869 \\
\hline $74(a)$ & 120.74628 & 1.94536 & 18.685608 & 10.185078 \\
\hline $74(b)$ & 95.133438 & 2.4785581 & 41.919707 & 9.2307692 \\
\hline $74(c)$ & 91.945412 & 2.3729123 & 42.18599 & 10.879121 \\
\hline $74(d)$ & 139.11363 & 1.3276207 & 43.839527 & 14.615385 \\
\hline 75 & 103.18802 & 1.4596217 & 15.796064 & 11.80555 \\
\hline $76(a)$ & 142.97789 & 2.5730292 & 20.378311 & 8.2897606 \\
\hline $76(\mathrm{~b})$ & 203.35707 & 1.0903724 & 22.154999 & 19.710411 \\
\hline $77(a)$ & 250.97209 & 1.1162667 & 20.156166 & 17.320984 \\
\hline $77(\mathrm{~b})$ & 161.33316 & 1.2586152 & 18.14994 & 14.472946 \\
\hline 78 & 75.051323 & 1.9595102 & 15.242311 & 8.3495941 \\
\hline 80 & 160.82599 & 1.8901941 & 20.464567 & 11.82225 \\
\hline 85 & 339.70535 & 3.4161331 & 42.541367 & 17.031494 \\
\hline 87 & 452.26419 & 1.9243208 & 37.445065 & 23.518646 \\
\hline 89 & 116.73711 & 1.6712568 & 15.861797 & 11.119179 \\
\hline 90 & 135.79277 & 2.1466852 & 20.178295 & 9.5460978 \\
\hline
\end{tabular}


The data of Table 8 is compared to the characteristics of the constituent particles found during the metallographic examination. Figure 48 shows the particle area distribution found during metallography (Figure 30 ) along with the nucleation site constituent particle data of Table 8. Clearly, the constituent particles associated with nucleation sites are in the upper range of the particle size range and the particles responsible for crack nucleation make up only a very small percentage of the population.

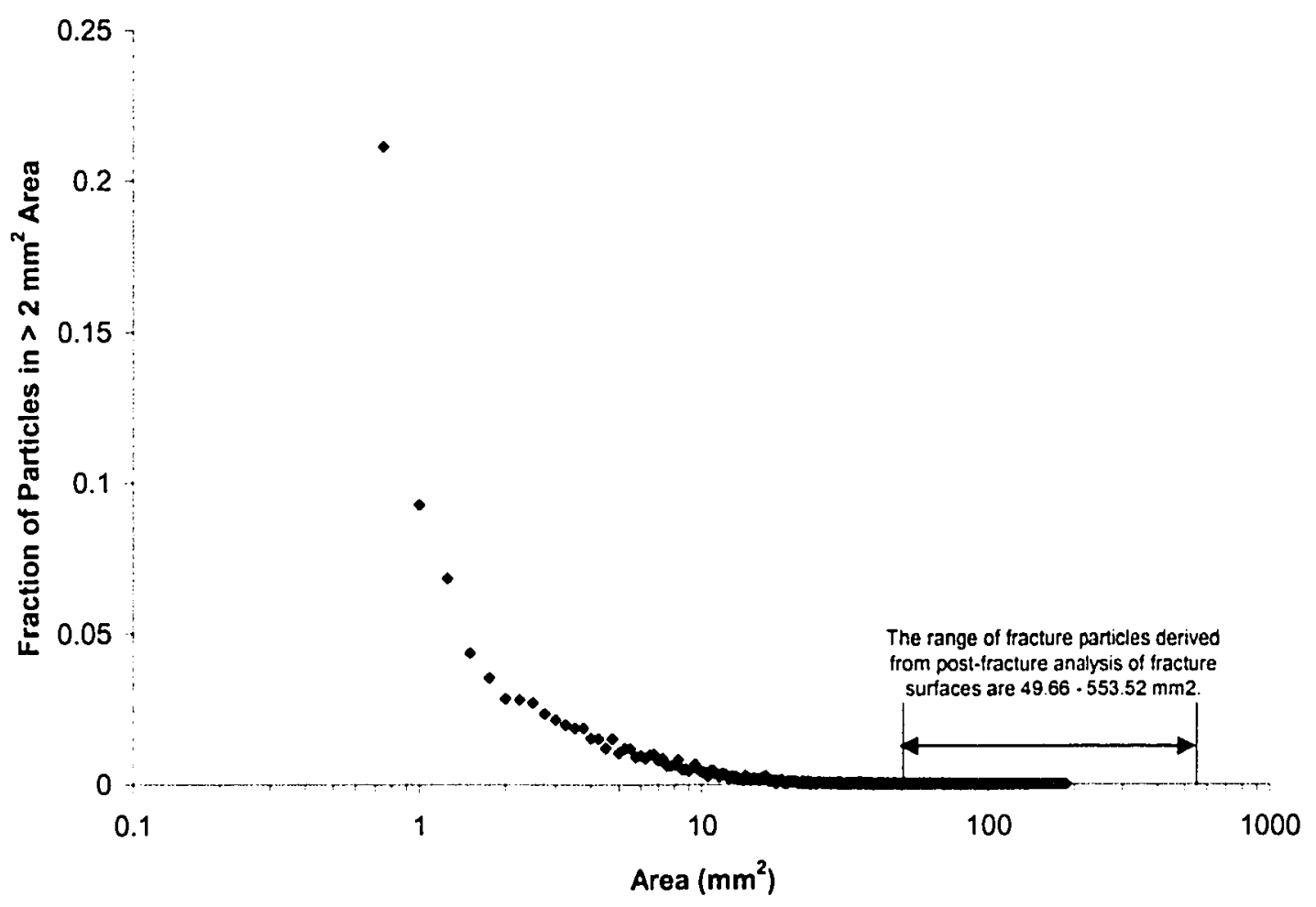

Figure 48. Particle area distribution in the ST plane with the nucleating particle data included

During post-fracture analysis, examination was also performed with the fracture surfaces tilted at a $30^{\circ}$ angle to reveal the condition of the notch wall. During this analysis, cracks were found on the side of the notch. Typical cracks found on the notch wall are shown in 
Figure 49. Figure 49a) shows one of the largest cracks found on the notch wall that did not ultimately lead to failure of the specimen. Figure $49 \mathrm{~b}$ ) shows a different sample with many cracks on the notch wall. Also, it should be noted that this micrograph was taken using the backscattered electron detector to confirm the involvement of the constituent particles on all cracks that nucleated during the fatigue testing. Figure 49c) and d) are high magnification pictures of typical cracks found on the notch wall and further demonstrates the involvement of constituent particles on the nucleation of fatigue cracks. Figure 49e) and $\mathrm{f}$ ) are taken at the notch tip from a sample that was only fatigued for approximately 17500 cycles to show the absence of cracks along the notch wall and to show that the cracks found in Figure 49a) to d) are a result of fatigue and not of processing or machining.

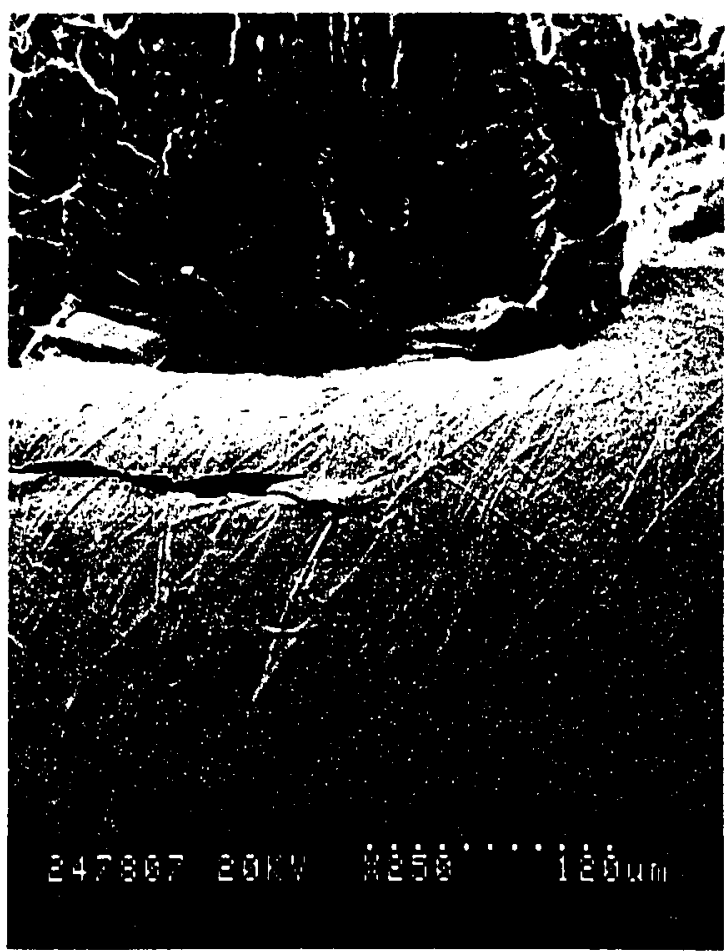

a)

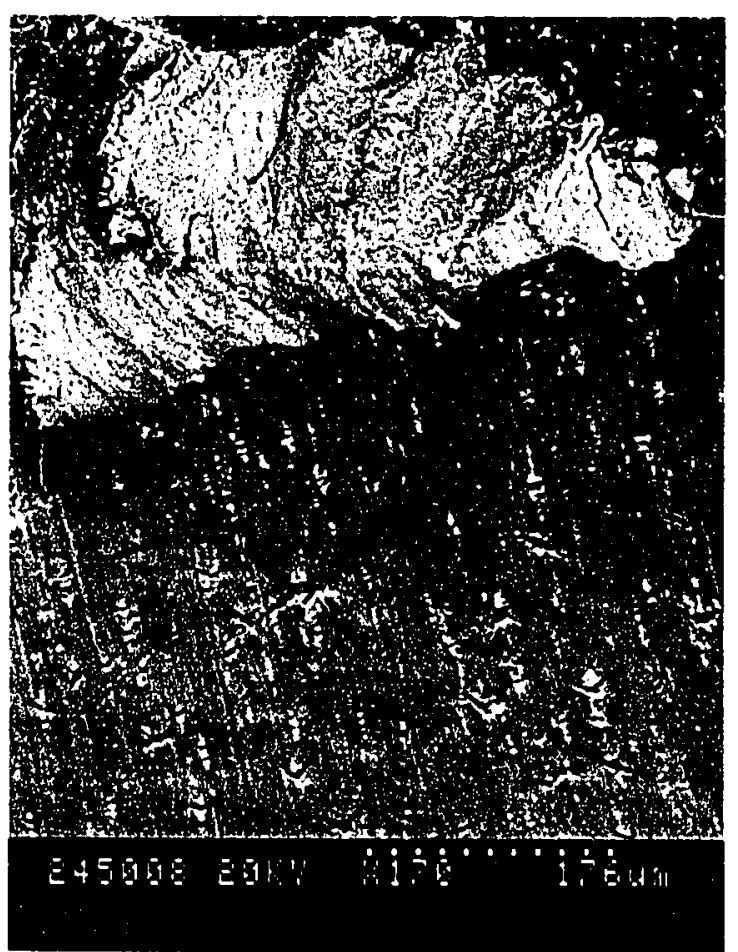

b) 

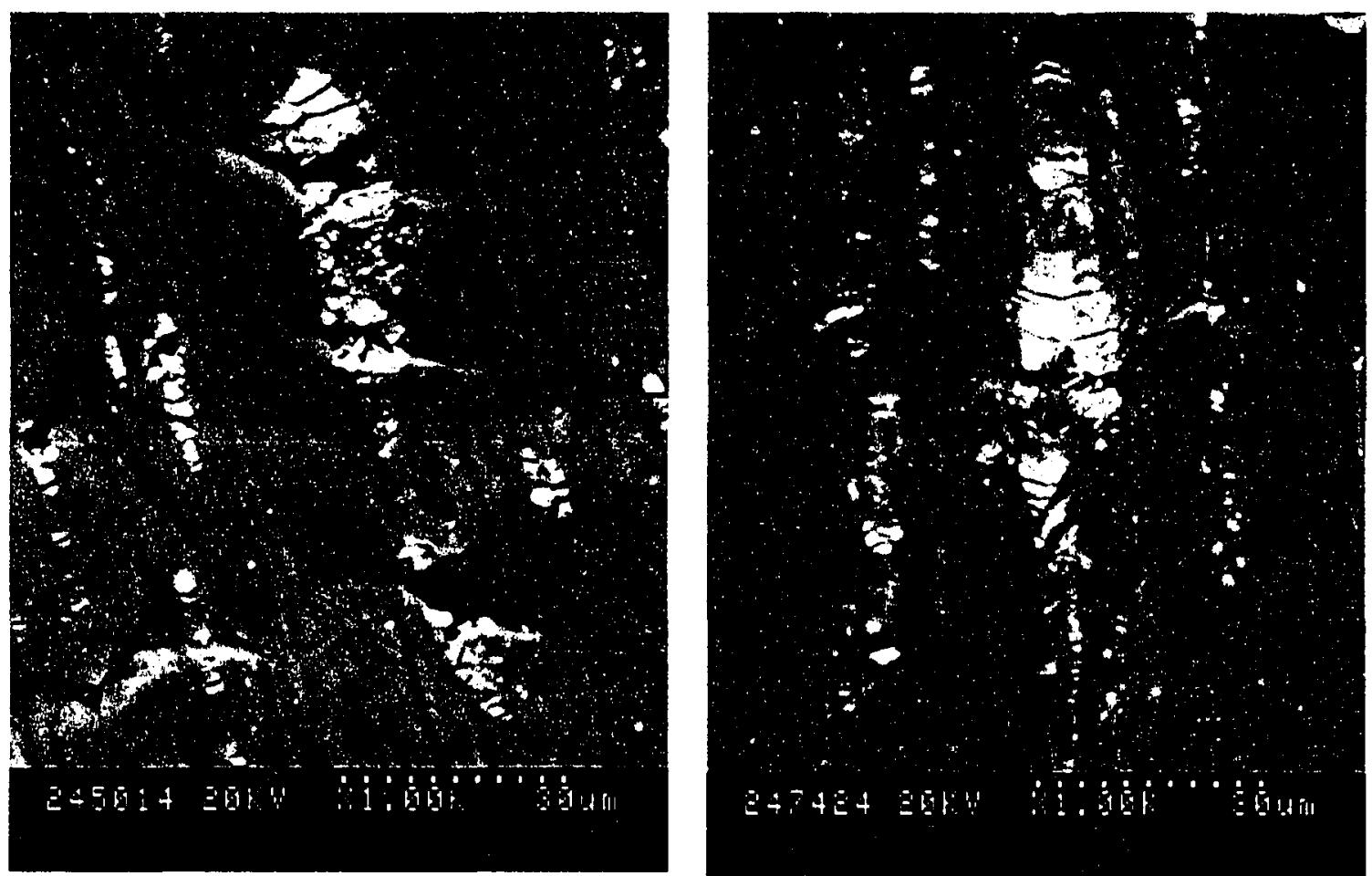

c)

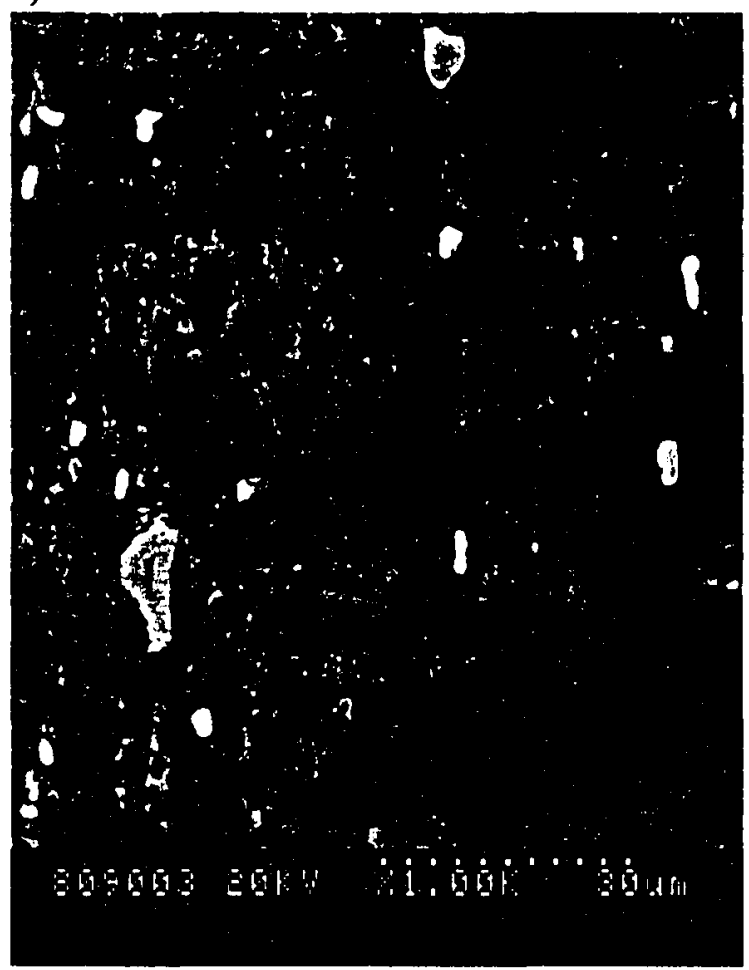

d)

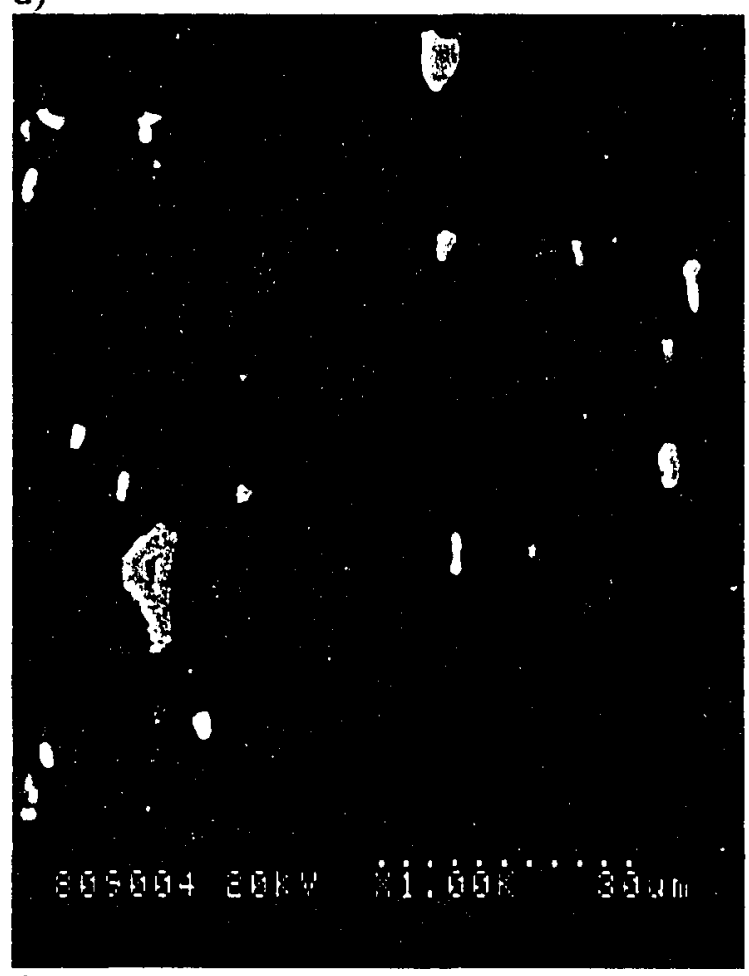

f)

e)

Figure 49. Examples of different cracks found along the notch wall taken with the a) secondary electron detector, b) backscattered electron detector, and also c) \& d) at high magnifications. Micrographs in e) and $f)$ are taken at the notch wall of a sample that was fatigue for $\sim 17500$ cycles, showing no cracking at the constituent particles. 
Based on the approximation of a circular crack front, it appears, from the surface crack size on the notch wall, that these cracks have arrested after growing to a maximum of only a couple of grain sizes as can be seen by Figure 49b). It is also seen that many cracks only nucleate and grow roughly to the size of a constituent particle, as shown in Figure 49c) and d). These findings have led to a hypothesis about crack nucleation and short fatigue crack growth patterns.

The theory behind crack nucleation and short growth with these new findings is that with the application of fatigue loads, many cracks will nucleate from constituent particles and be arrested when they reach the nearest microstructural barrier such as a grain boundary. A grain boundary can act as a barrier to crack growth if the neighbouring grain is unfavourably orientated. The crack will not continue to grow until a certain threshold stress is met. In some cases, the crack will continue to growth after the first grain boundary while others will be arrested, depending on the length of the first grain and the orientation of the neighbouring grain. At some point however (usually within the first two or three grains), all of the cracks are halted at a grain boundary. This hypothesis is further strengthened by the findings of Blom et al. [7] who also found that the growth rate of short cracks slows down drastically as it approached a grain boundary, as seen in Figure 50.

The crack that eventually leads to fatigue failure of the material is the one crack that, after many fatigue cycles, eventually breaks through the retarding grain boundary and continues to grow to exhibit properties of long cracks. In the long crack regime, the 
crack has grown long enough and the $\Delta \mathrm{K}$ is high enough that grain boundaries no longer impede the growth of the crack. This hypothesis is shown schematically in Figure 51 and Figure 52 where Figure 51 shows the overall hypothesis of fatigue crack nucleation and growth, and Figure 52 shows specifically the crack that eventually leads to fatigue failure.

Figure 51 shows schematically that all of the large constituent particles along the notch wall will become cracked during fatigue testing but most cracks will be arrested by high angle grain boundaries. Then, after continuous fatigue loading, one of these cracks will break through and continue to grow until the stress surpasses the tensile strength of the material.

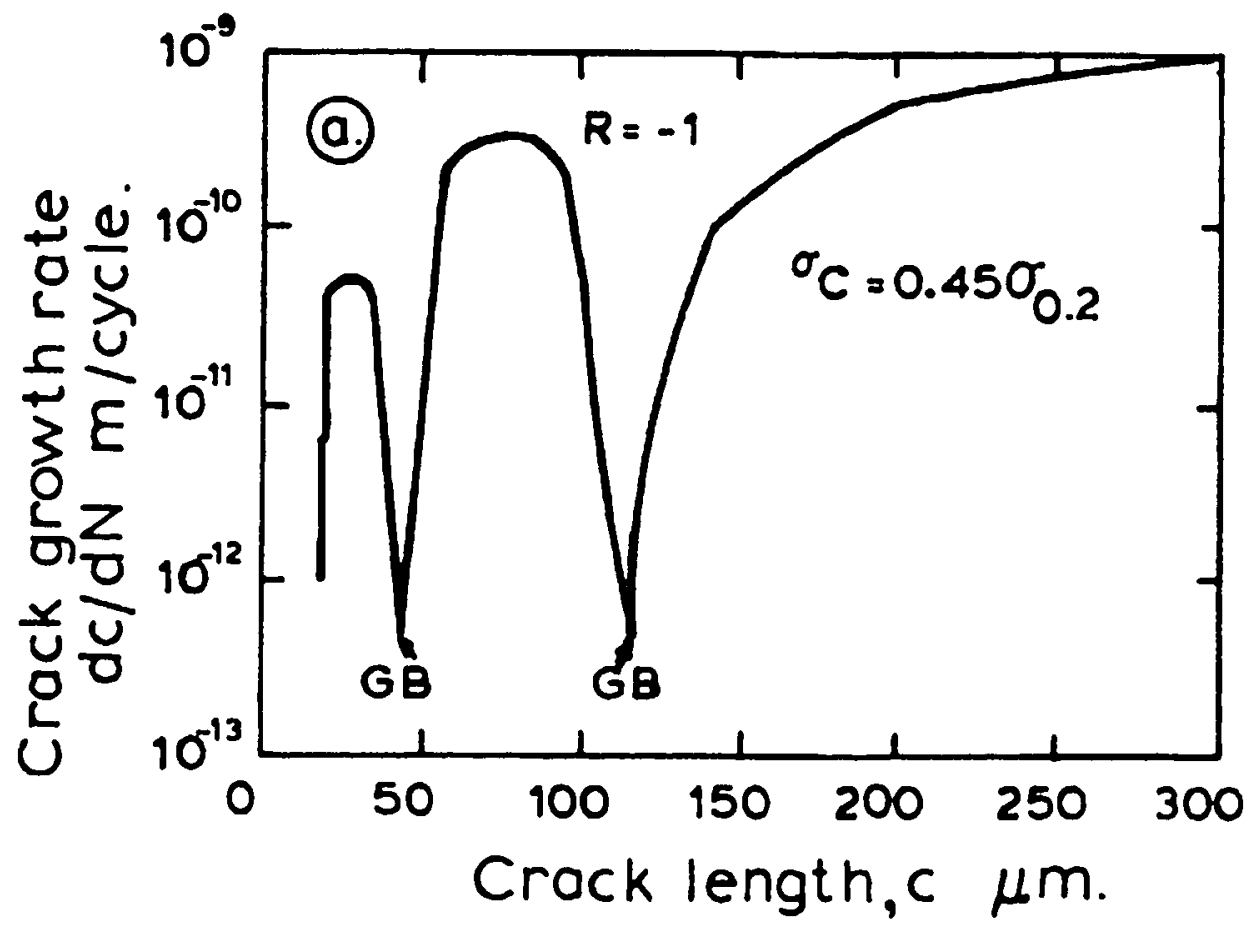

Figure 50. Crack growth rate vs. crack length $|7|$ 


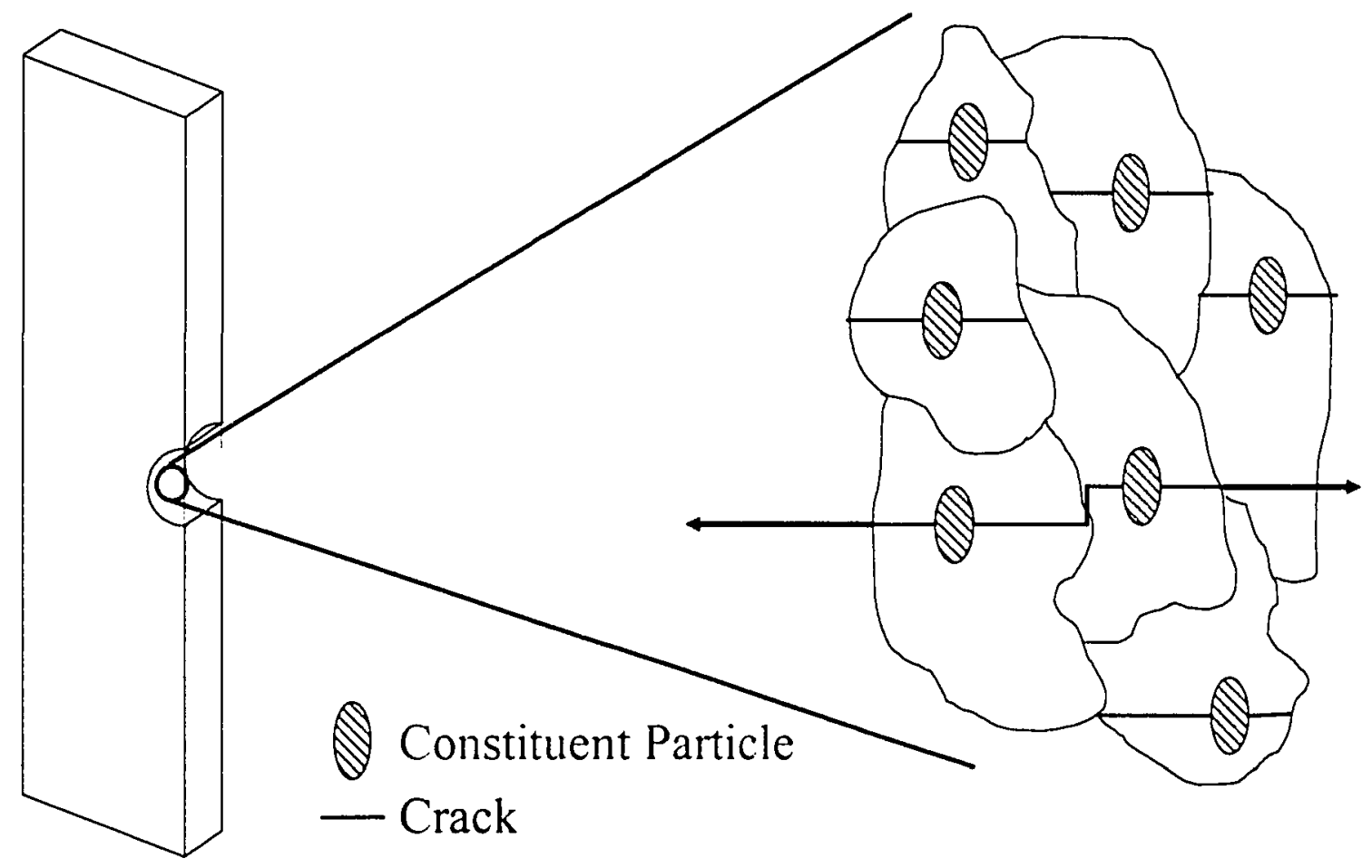

Figure 51. Schematic of proposed hypothesis on the notch wall

Figure 52 shows the progression of the crack on the fracture surface. The crack nucleates from the constituent particle (which is depicted by the striped red object) and then grows rapidly until it reaches the first set of barriers (grain boundaries in this case), at which point, it may or may not get arrested depending on the orientation of the next grain. Once the stress threshold has been met, the crack will continue to propagate until it reaches the next set of barriers where it will once again be halted. It should be noted that in this stage, there is no unified crack front and each grain's crack growth rate, direction and behaviour are independent of the others. Once a crack is able to break through the barrier, it is long enough and the $\Delta \mathrm{K}$ is large enough that it will enter the long crack regime and continue to grow until failure. 


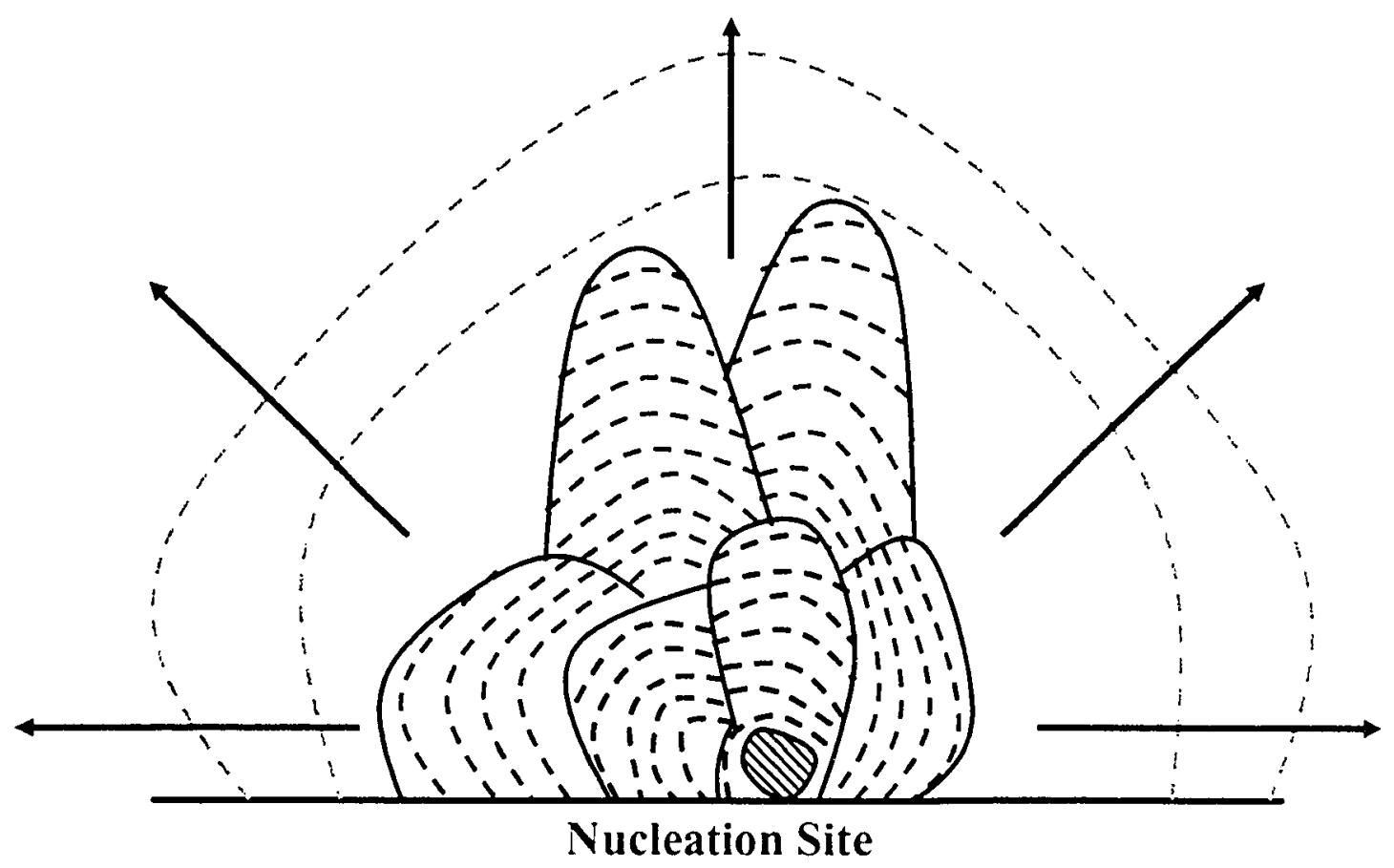

Figure 52. Crack nucleation and growth hypothesis of a crack that eventually leads to failure

The reason why it has been found that there is no correlation between the nucleation size and fatigue performance is because the barriers to crack growth (grain boundaries) play the primary role in the short crack regime. The biggest factor controlling the time spent in the nucleation and short fatigue crack regime is how long the crack spends being impinged by the barrier, not the IDS size as originally thought. Consequently, because these two regimes consume up to $95 \%$ of the fatigue life, the factor controlling the nucleation and short crack regime will also ultimately have the greatest impact on the overall fatigue life.

This is similar to the results found by Merati et al. [3][4][5] who found that even at low stress levels, short cracks grew and developed within the matrix on coarse slip lines radiating from constituent particles and were heavily influenced by the presence of grain 
boundaries. The reason offered for the influence of grain boundaries was that the plastic zone ahead of the crack tip was so small that it was confined within a grain and its boundaries and may not be transmitted into the neighbouring grain.

Also, Navarro and de los Rios [30], who modeled the fatigue crack growth rate using different criteria for the long and short crack regimes, considered the growth of short cracks in terms of successive blocking of the plastic zone by slip barriers (i.e. grain boundaries) and the growth of a crack in the neighbouring grain. The factor governing the movement of slip across a slip barrier is the stress concentration ahead of the plastic zone, which is controlled by the crack size. This discontinuous crack behaviour used by Navarro and de los Rios [30] to govern the short crack regime is proved by the existence of the arrested cracks found along the notch wall during scanning electron microscopy examination of the fracture surface.

Blom et al. [7] and Lankford [29] also found supporting evidence for such a mechanism of crack growth. Blom et al. [7] observed that the crack growth process was not continuous and, in fact, the crack growth rate decreased as it approached a grain boundary. They also showed that a grain boundary, which is a barrier to slip propagation, would dictate the threshold for further crack growth. Similarly, Lankford [29] found evidence of crack nucleation in the second grain after the initial crack had arrested at the grain boundary and a gradually increasing crack growth rate after the crack has surpassed the third grain, thereby implying the transformation from short to long crack after the third grain. 
Based on the importance of grain boundaries in the short crack regime, it can be deduced that the grain size must play an influential role in the fatigue properties of 2024-T3. Starke et al. [38] suggested that a smaller grain size will decrease the slip length and pileup stress because they found that the number of cycles to crack nucleation was much higher for a finer grained material with an identical yield strength due to less intense slip bands. Another study by Lutjering and Gysler [39] also showed improved fatigue life and a higher number of cycles before crack nucleation due to a grain size reduction of the same material. This is supported by the fact that larger grains have been known to facilitate dislocation pileup and decrease the pileup stress needed to break through the grain boundary and transfer slip to the neighbouring grain. Merati et al. [3][4][5] explained that the relationship between the grain size and the stress required to initiate or propagate slip is similar to the Hall-Petch relationship;

$$
\sigma=\sigma_{0}+k D^{-\frac{1}{2}}
$$

where $\sigma_{0}=$ flow stress,

$\sigma=$ stress required for slip initiation or propagation,

$\mathrm{k}=\mathrm{a}$ constant, and

$\mathrm{D}=$ grain size

Formerly, Merati et al. [3][4][5] also found that one of the largest parts of crack nucleation and short crack growth is the time spent to transfer slip across the grain 
boundaries of the first and second grains. As a result, the reason why no correlation was found between fatigue life and the initial discontinuity size was that the sizes of the first and second grains, which dominate the time spent in the short crack regime, mask the effect of the size of the initial discontinuity on fatigue life.

\subsection{Energy Dispersive X-ray (EDX) Analysis}

An energy dispersive $x$-ray (EDX) analysis was conducted using a SEM in order to identify the chemical composition of the constituent particles that acted as fatigue crack origins and the ones that did not play any role in fatigue crack nucleation so that they may be compared and the differences can be observed.

Many particles of varying size and shape observed from the metallographic analysis were chemically examined as well as the large particles found at the crack nucleation site to investigate whether there was a difference between the particles that initiate fatigue cracks and those that do not.

The EDX analysis conducted returned similar results as those found by Merati et al. $[3][4][5]$. Figure 53 shows the EDX analysis of the 2024-T3 matrix and it follows very closely, as expected, with the standard composition for the 2024 set by the ASM standards [36] 


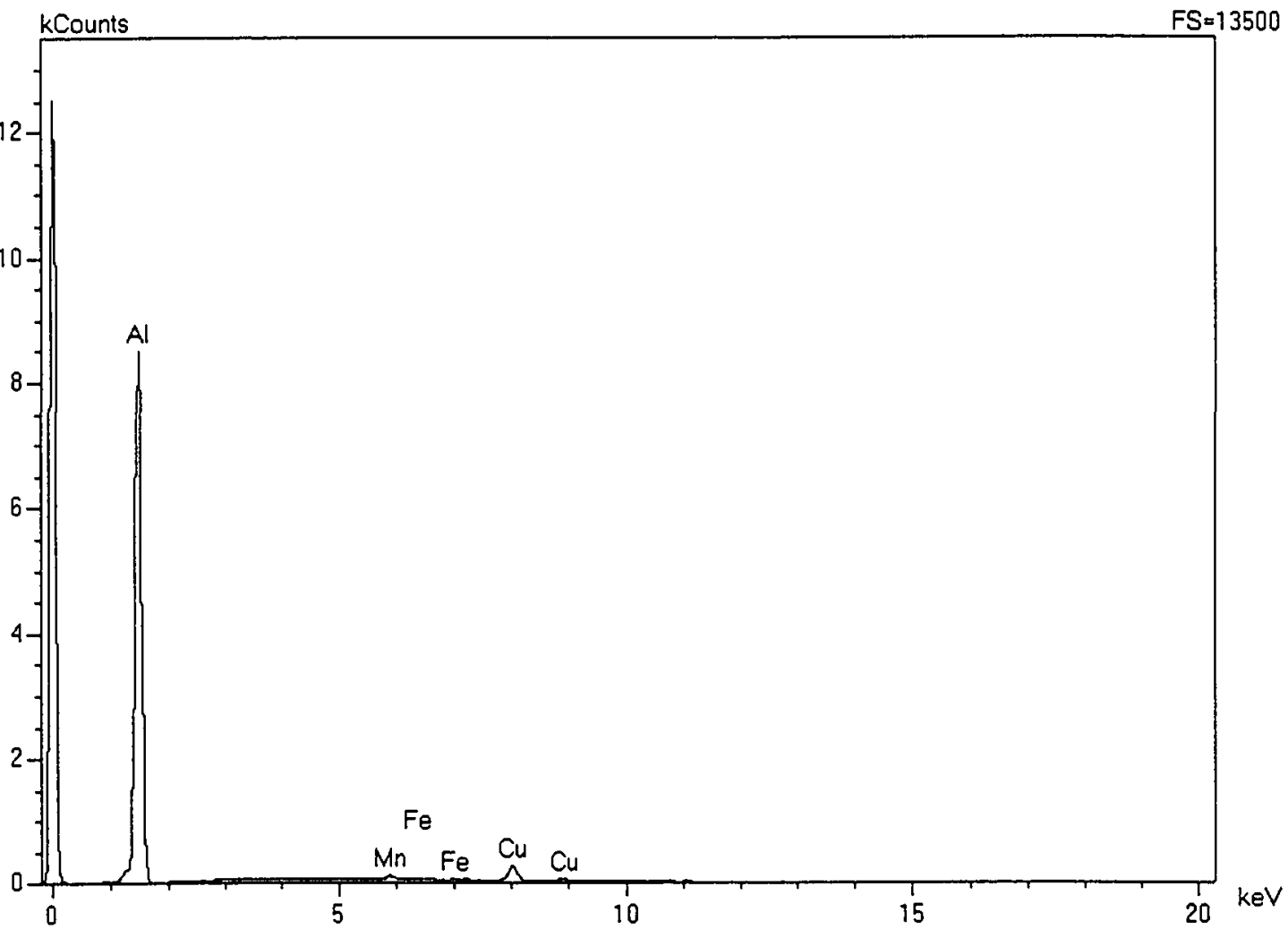

Figure 53. Chemical EDX analysis of the 2024-T3 aluminum matrix

Figure 54 to Figure 56 show the typical EDX analysis results for the small particles (less than $6 \mu \mathrm{m}^{2}$ in size) that were found to have no bearing on fatigue crack nucleation, the large particles found during metallography whose size is similar to those found at the nucleation site of the fatigue samples, and the large particles found at the nucleation site of the fatigue samples tested in this project, respectively. The approximate average quantitative chemical composition of each phase is also shown in Table 9. As can be seen, the EDX analysis for the large particles found during metallography and the ones found at the fatigue crack nucleation sites are virtually identical. Therefore, this indicates that the large particles examined during the metallographic analysis portion of this project are the same as the ones that have been found to be the main culprit for fatigue crack nucleation. However, there are significant differences between the chemical composition 
of the large particles and the smaller particles that are less than approximately $6 \mu \mathrm{m}^{2}$ in size. As can be seen from the figures below, the larger particles contain iron and manganese where as the smaller particles do not. It can also be seen from Table 9 that the larger particles also contain significantly more copper. It should be noted that based on the composition of 2024 , the small and large particles will also contain trace amounts of other elemental additions such as chromium and magnesium that are below the detection limit of the EDX system. Based on the typical phases seen in 2024 as described in the ASM handbook, some of the possible phases for the large particles could be $\mathrm{Cu}_{2} \mathrm{FeAl}_{7}$ and $\mathrm{Al}_{12}(\mathrm{Fe}, \mathrm{Mn})_{3} \mathrm{Si}$.

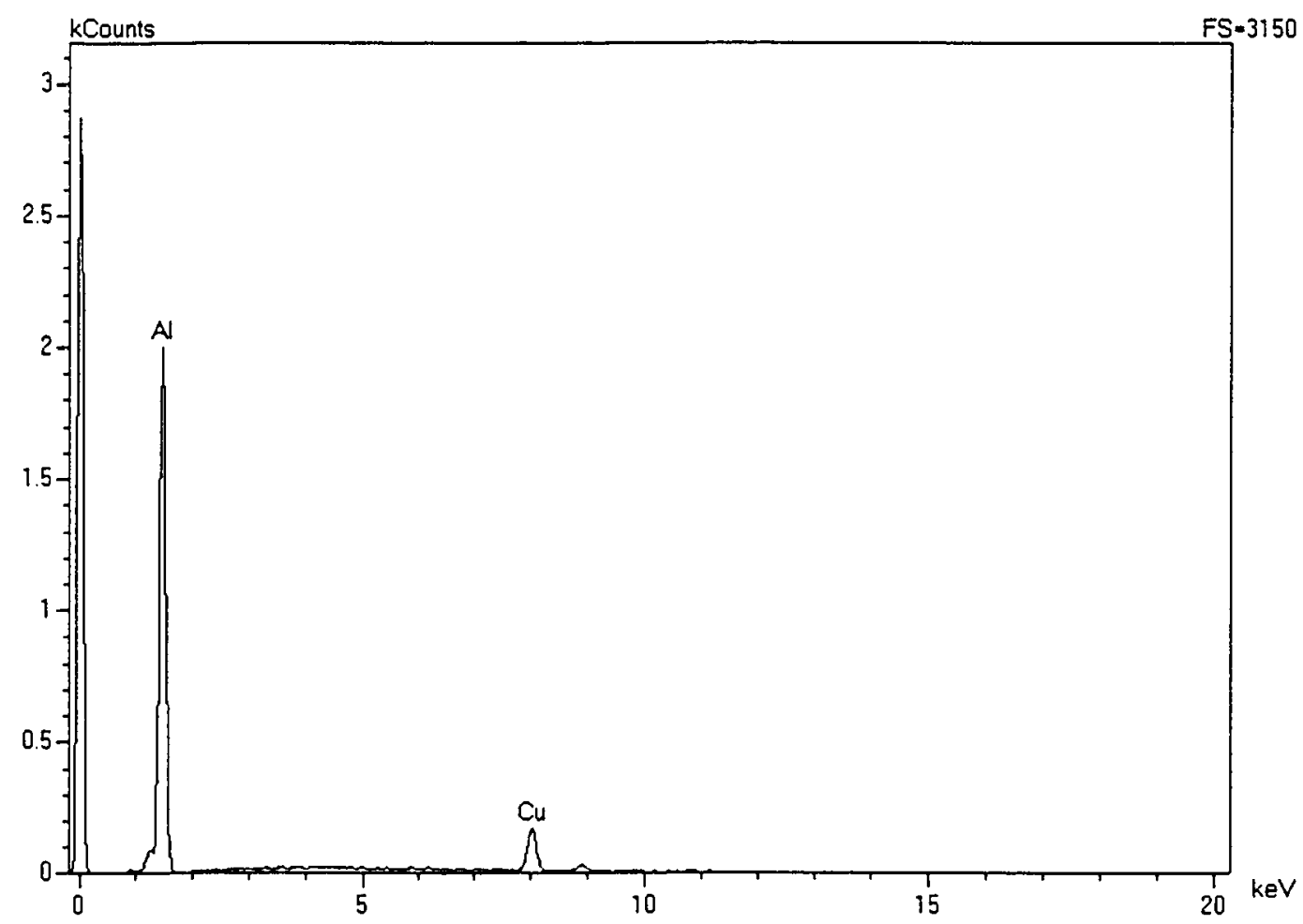

Figure 54. Typical EDX analysis of small particles found in metallography 


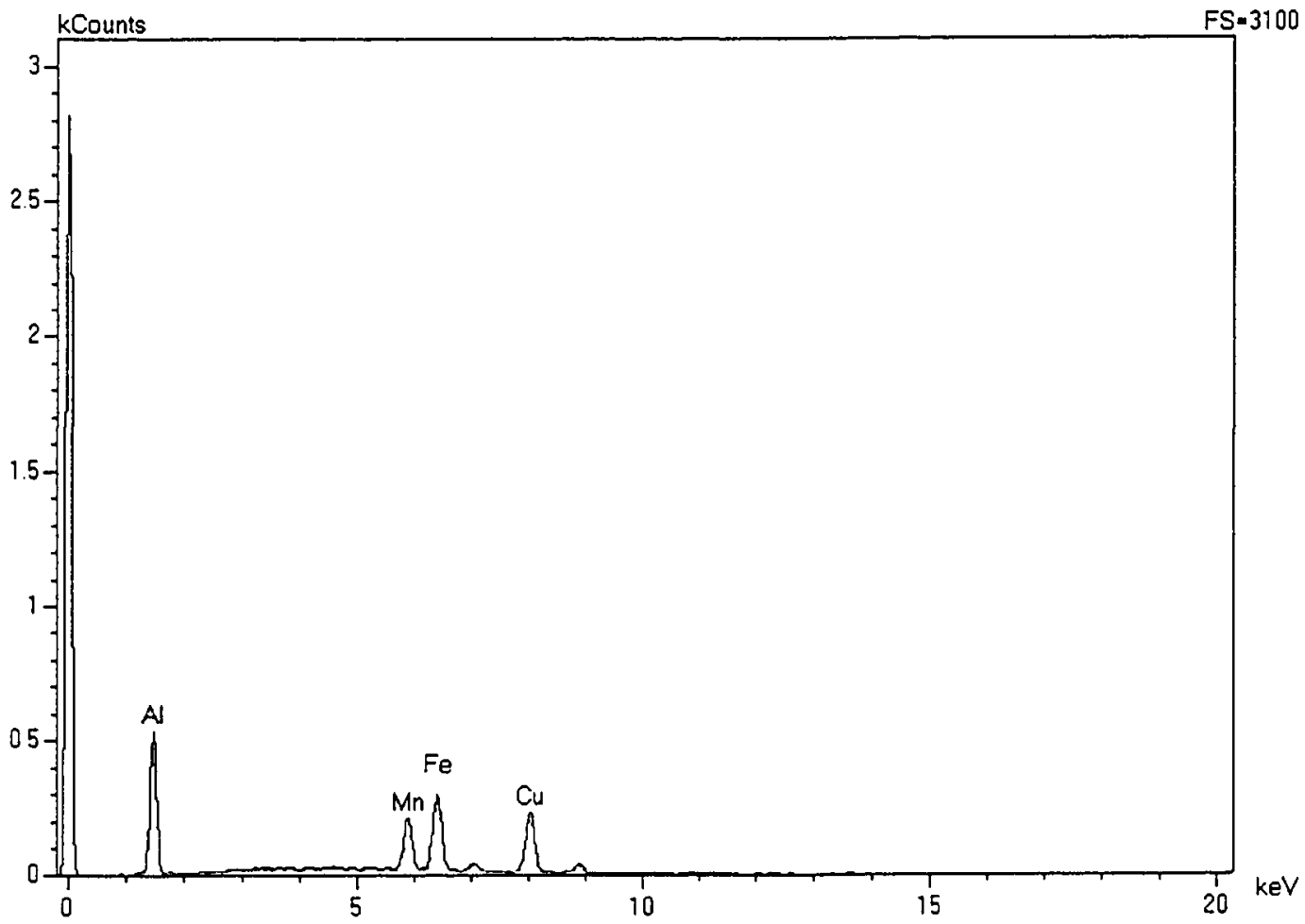

Figure 55. Typical EDX analysis of large particles found during metallography

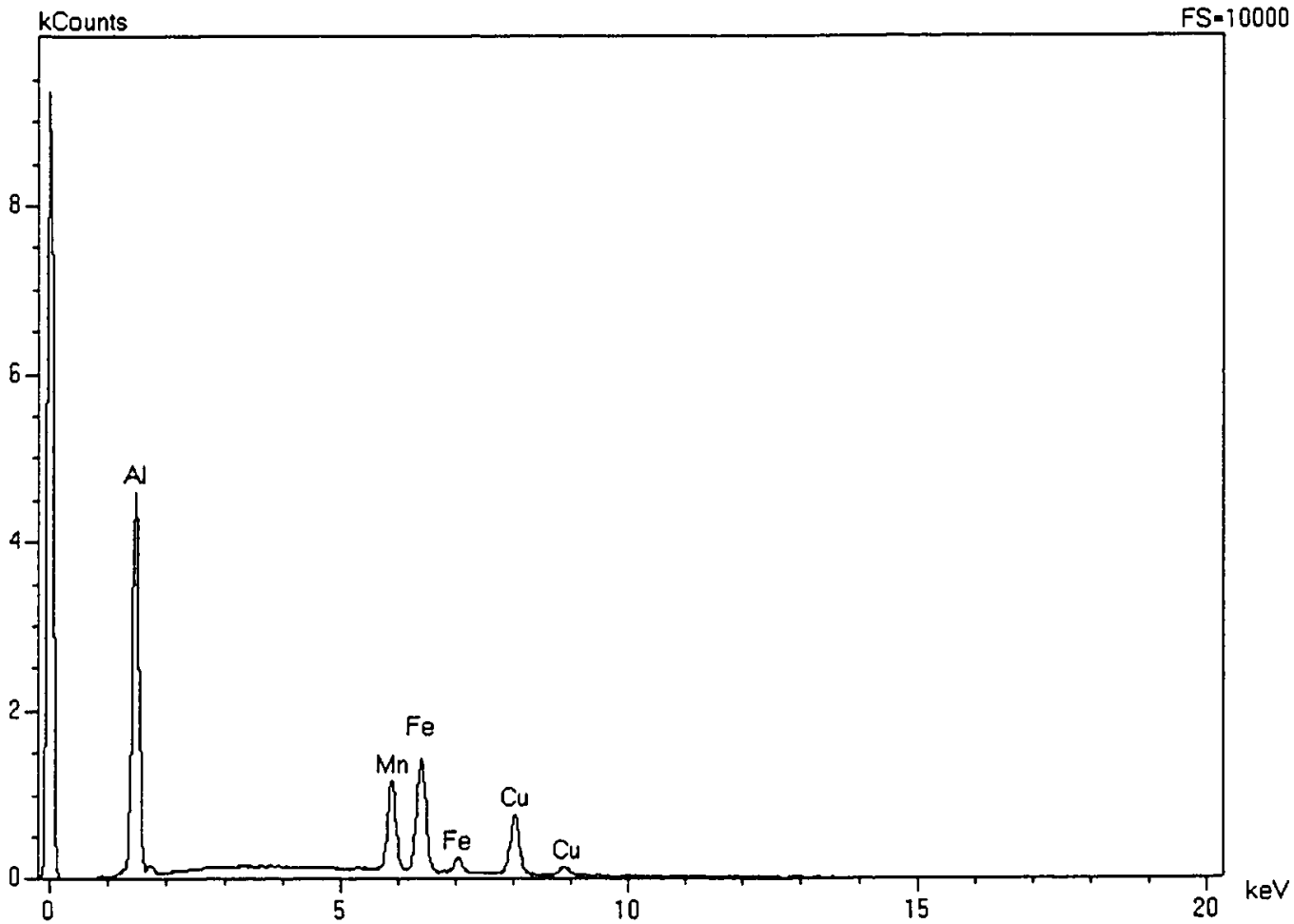

Figure 56. Typical EDX analysis of large particles found at the nucleation site on the fracture surface 
Table 9. Approximate average chemical composition of each phase examined during the EDX analysis

\begin{tabular}{|c|c|c|c|c|c|c|c|c|}
\hline & \multicolumn{3}{|c|}{ Weight \% (Average and approximate) } & \multicolumn{3}{c|}{ Atom \% (Average and approximate) } \\
\cline { 2 - 10 } & $\mathrm{Al}$ & $\mathrm{Mn}$ & $\mathrm{Fe}$ & $\mathrm{Cu}$ & $\mathrm{Al}$ & $\mathrm{Mn}$ & $\mathrm{Fe}$ & $\mathrm{Cu}$ \\
\hline 2024-T3 Matrix & 95 & 0.5 & 0.1 & 4 & 98 & 0.25 & 0.05 & 1.5 \\
\hline Small Particles & 91 & 0 & 0 & 9 & 96 & 0 & 0 & 4 \\
\hline Large Particles & 70 & 6.5 & 7 & 16 & 84 & 4 & 4 & 8 \\
\hline Nucleation Particles & 70 & 6.5 & 7 & 16 & 84 & 4 & 4 & 8 \\
\hline
\end{tabular}




\subsection{CONCLUSION}

A metallographic analysis was performed on the candidate 2024-T3 to characterize the constituent particles that were found to have an influential role in fatigue crack nucleation. One of the main metallographic findings was that the constituent particle population was found to follow a Weibull distribution. This shows that the particles that were found at the fatigue crack nucleation sites $\left(>50 \mu \mathrm{m}^{2}\right)$ only make up a very small percentage $(<0.5 \%)$ of the total constituent particle population. Another major finding was that the larger constituent particles that were of the same size as those found at the nucleation site were generally found in the mid-thickness of the aluminum sheet. This finding further confirms the belief that the large constituent particles play the most vital role in fatigue crack nucleation of the bare 2024-T3 aluminum alloy because all of the fatigue failures in this project nucleated from the mid-thickness region.

A total of over 70 fatigue tests were performed in this thesis in order to evaluate three different short crack detection methods; surface replication, marker bands, and electrical potential drop (EPD). The surface replication method was evaluated and it was found that this method is not suitable or efficient for detecting short fatigue cracks. Even though the surface replicas are suppose to have resolution down to $0.1 \mu \mathrm{m}$, the replications were not sensitive enough to catch the fatigue crack when it was still in the short crack regime. By the time the fatigue crack was visible, it was already in the long crack regime. In addition, the replication technique is very time and material consuming, which increase operational costs and greatly decrease the efficiency of this process. 
The marker band technique, while very useful to the EPD technique, was also found to not be suitable for detecting short cracks. The marker bands on the fracture surface are there as a result of varying the maximum load applied during the cyclic fatigue process and thereby periodically changing the fatigue crack growth rate and creating "bands" or "beachmarks" on the fracture surface. The marker band technique is slightly different than the other two techniques examined, in that, any information that this technique provides is only found during post-fracture analysis and not actually during the test. As a result, it already has the disadvantage in that it won't be able to provide any information during the actual fatigue test. It can however provide other useful information such as the crack size at a particular cycle count, the crack front shape at different stages of crack growth and, by measuring the space between each marker band, an approximation of the crack growth rate at that particular point of the fatigue life. The marker band technique was applied to all of the fatigue specimens tested in this program, including those used for the surface replication and EPD techniques, and it was found that in all of the cases, no marker bands were found in the short crack regime. One of the possible reasons why is that the crack growth process in the short crack regime is not continuous and occurs so quickly that the marker band technique can not apply distinguishable marker bands that can be found through optical or scanning electron microscopy. Another reason may be that when the crack has reached into the long crack regime, crack closure affects actually cause the two sides of the fracture surface to touch and it may "rub off" the fine marker bands in the short crack regime. 
The EPD method using a DC power source was the last technique to be evaluated. This technique involved sending a current through the notch of the fatigue specimen and the potential drop across the notch of the specimen is measured. The presence of a crack alters the current flow lines and as such, also alters the electric field produced by the flow of the current through the sample. This change in the electric field produces an increase in the potential drop and, through a secondary crack measurement technique, a correlation can be made between the potential drop and the crack size. During various fatigue tests involving the EPD method, different current and gain levels were examined to find the optimal settings in this technique, which was $12 \mathrm{~A}$ of current and a gain of $8,000 \mathrm{X}$. Using these optimal settings, a crack as small as $42 \mu \mathrm{m}$ in radius was detected which is quite remarkable considering the high conductivity of aluminum alloys that greatly hinder its ability to be used with any potential drop techniques.

A post-fracture analysis was performed on all of the fatigued samples in order to closely examine any findings in the nucleation and short crack regime. It was found, using the backscattered electron detector, that all of the fatigue cracks nucleated from large constituent particles. All of the constituent particles were analyzed and measured, in a technique similar to the one used during metallography, and it was seen that the size of these particles range from $\sim 50 \mu \mathrm{m}^{2}$ to $\sim 550 \mu \mathrm{m}^{2}$.

Another important finding during the post-fracture analysis was there were many other small cracks found along the notch wall, all of which were arrested and did not contribute to the failure of the fatigue specimen. Upon further examination, it was also discovered 
that these cracks nucleated from constituent particles as well, although in many cases, the particles were smaller than the $50 \mu \mathrm{m}^{2}$ lower size limit observed on the fracture surface. This important finding led to the hypothesis that during the fatigue testing, many cracks will nucleate and become arrested, in succession, when it reaches the first unfavourably oriented grain boundary. This process will continue until the stress intensity $(\Delta \mathrm{K})$ becomes large enough to surpass the threshold level and one of the cracks will break through the grain boundary barrier, transform into a long crack and eventually lead to fatigue failure. Therefore, the time spent in the nucleation and short crack regimes, which made up a majority (sometimes up to $95 \%$ ) of the fatigue life, is a result of the nucleation and short crack propagation of not just one crack, but many cracks. That is why such a large portion of the fatigue life is spent in those two regimes.

A chemical analysis using the energy dispersive $x$-ray analyzer was also performed on the particles that were found at the nucleation site and the ones that were not in order to examine any chemical differences between the two. It was found, by also examining the larger particles found during metallography, that they are the same particles as those found at the crack nucleation sites. It was found that the difference between the large and small particles was that the larger particles contained iron and manganese whereas the smaller particles did not. In addition, the larger particles also contained significantly more copper than the smaller particles. It is believed that all iron and manganese-bearing particles will nucleate cracks upon fatigue loading. As a result, if the iron content, which is the most abundant impurity in 2024 , was lowered, the fatigue life would be extended. The main drawback to this would be the additional cost of higher purity. 
From this thesis, the most important contributions are:

1) the EPD technique proved to be the most successful short crack detection technique, detecting cracks as small as $43 \mu \mathrm{m}$ in radius,

2) fatigue crack nucleation is controlled by iron and manganese-bearing constituent particles but short cracks are controlled by grain size and orientation,

3) 2024's crack nucleation behaviour can be improved by reducing the amount of iron, and

4) 2024's short crack behaviour can be improved by controlling the grain size and orientation relationships. 


\subsection{FUTURE WORK}

While a lot of effort has been put into evaluating the surface replication, marker band and EPD (electrical potential drop) techniques for detecting short fatigue cracks in the 2024T3 aluminum alloy, the ACPD (alternating current potential drop) method was not evaluated in this project due to time, material, and equipment constraints. It is often argued that the ACPD methodology would be better than using direct current, which is the case in the EPD testing in this thesis, for the detection of short fatigue cracks because of the "skin" effect produced. For the ACPD technique, a constant amplitude (normally sinusoidal) current is passed through the specimen to generate a voltage drop across the crack. It has been observed that when the current is passed through the current at a high frequency, the current distribution across the thickness of the sample becomes nonuniform and the current tends to be carried only near the surface of the specimen. This term is called the "skin" effect and allows the ACPD method to be more sensitive to cracks that occur on the surface of the specimen.

In addition, similar tests need to be performed on 2124 , which is an alloy very similar to 2024 except that it contains fewer impurities such as iron. Based on the conclusions of this thesis, 2124 should have better crack nucleation behaviour but the same short crack growth behaviour, assuming that the 2124 has a similar grain size to the 2024 tested in this thesis. This evaluation would give further credence to the conclusions of this thesis. 
The detection methods evaluated in this study could be used in more detailed examinations of the short crack regime in high strength aluminum alloys such as 2024-T3 where the hypotheses proposed in this project can be scrutinized and studied. Once the controlling mechanisms and properties of the short crack regime are revealed and proven, it can be incorporated into life prediction methodologies. With the nucleation and short crack regime data incorporated into the existing life prediction methodologies, a holistic life prediction methodology can finally be established that encompasses all the stages and regimes of the fatigue life of high strength aluminum alloys. This holistic fatigue methodology will no longer have to rely on assumptions and estimations, which limited the model's application to only the situations where these assumptions were valid. With a complete life prediction methodology that is applicable to multiple scenarios, the model can then be modified to include other factors that affect the material's service life such as corrosion fatigue and fretting fatigue. Developing such a complete model will allow a more universal methodology to be used in the management of in-service aircraft and will have many commercial benefits. 


\subsection{REFERENCES}

1. T. Swift, "Damage Tolerance Capability", International Journal of Fatigue, Vol. 16, pg. 75-94, 1994.

2. Hercules Structural Repair Manual, Lockheed-Georgia Company, A Division of Lockheed Corporation, SMP583-51-1-003, 1980.

3. A.A. Merati, J. Tsang, S. Khomusi, A. Kourline, K. Kyle, and M. Geusebroek, "Preliminary Report - Final Test Results for the Determination of FatigueRelated Features of the Initial Discontinuity State (IDS) of 2024-T3 Aluminum Alloy", NRC/IAR Report LTR-SMPL-2002-0110, May 2002.

4. A.A. Merati, J. Tsang, and K. Kyle, "Exploratory Test Results for the Determination of Fatigue-Related Features of the Initial Discontinuity State (IDS) of Two Aluminum Alloys", NRC/IAR Report LTR-SMPL-2001-0150, July 2001.

5. A.A. Merati, "Test Procedure for the Determination of Fatigue-Related Features of the Initial Discontinuity State (IDS) of Candidate Aluminum Alloy Components for the USAF/Lockheed Martin CFSD Phase 2 Project", NRC/IAR Report LTR-SMPL-2001-0035, February 2001.

6. M. Canton, "Predicting Fatigue Properties of Cast Aluminum by Characterizating Small-Crack Propagation Behavior", Ph.D. Thesis, University of Michigan, 2001.

7. A.F. Blom, A. Hedlund, W. Zhao, A. Fathulla, B. Weiss, and R. Stickler, "Short Fatigue Crack Growth Behaviour in Al 2024 and Al 7475," The Behaviour of Short Fatigue Cracks, EGF Pub 1 (Edited by K.J. Miller and E.R. de los Rios), 1986, Mechanical Engineering Publications, London, pg. 37-66. 
8. M.H. Swain, J.C. Newman, Jr., E.P. Phillips, and R.A. Everett, "Fatigue Crack Initiation and Small Crack Growth In Several Airframe Alloys," Third International Conference on Fatigue and Fatigue Thresholds, Engineering Materials Advisory Services, ltd, 1987, pp. 1079-1084.

9. S.A. Barter, P.K. Sharp, G.Holden, and G. Clark, "Initiation and Early Growth of Fatigue Cracks in an Aerospace Aluminum Alloy," Airframes and Engines Division, DSTO Aeronautical and Maritime Research Laboratory, Melbourne, Australia, September 2001.

10. A.J. McEvily and Z. Yang, "An Analysis of the Rate of Growth of Short Fatigue Cracks," Short Fatigue Cracks, ESIS 13 (Edited by K.J. Miller and E.R. de los Rios), 1992, Mechanical Engineering Publications, London, pp. 439-448.

11. X.D. Li, "On Kinetics of Small Fatigue Crack Growth," Ph.D. Thesis, The Open University, 1996.

12. M.W. Brown, "Interfaces Between Short, Long and Non-propagating Cracks," The Behaviour of Short Fatigue Cracks, EGF Pub. 1 (Edited by K.J. Miller and E.R. de los Rios), 1986, Mechanical Publications, London, pp. 423-439.

13. A. Plumtree, B. P. D. O'Connor, "Influence of Microstructure on Short Fatigue Crack Growth," Fatigue and Fracture of Engineering Materials and Structures. Vol. 14, no. 2-3, pp. 171-184, 1991.

14. B.M. Hillberry and E.A. DeBartolo, "Effects of Constituent Particle Clusters on Fatigue Behaviour of 2024-T3 Aluminum Alloy," International Journal of Fatigue, Elsevier Science Ltd., Vol. 20, No. 10, November 1998, pp. 727-735. 
15. Y. Akiniwa, K. Tanaka, and E. Matsui, "Statistical Characteristics of Propagation of Small Fatigue Cracks in Smooth Specimens of Aluminum Alloy 2024-T3," Materials Science and Engineering, A104, April 1988, pp. 105-115.

16. M. Goto, "Scatter Characteristics of Fatigue Life and the Behaviour of Small Cracks," Fatigue and Fracture of Engineering Materials and Structures. Vol. 15, no. 10 , pg. $953-963$. Oct. 1992.

17. H. Kitagawa, and S. Takahashi, "Applicability of Fracture Mechanics to Very Small Cracks", Proc. $2^{\text {nd }}$ Int. Conf. on Mech. Behaviour of Materials, Boston, 1976.

18. W. G. Fricke Jr. and M. S. Hunter, "Study of crack initiation phenomena associated with stress corrosion of aluminum alloys Final summary report (Crack initiation associated with stress corrosion of aluminum alloys)," NASA-CR102474, Pagination 306P, 1969.

19. W. G. Fricke Jr. and M. S. Hunter, "Study of crack-initiation phenomena associated with stress corrosion of aluminum alloys Annual report, 6 Jun. 1967 - 6 Jun. 1968 (Electron microscopic, fractographic, and microprobe observations of crack initiation and propagation phenomena associated with stress corrosion of aluminum alloys),"NASA-CR-98161, AR-2, Pagination 186P, 1968.

20. J. C. Grosskreutz and G. G. Shaw, "Critical Mechanisms in the Development of Fatigue Cracks in 2024-T4 Aluminum," Fatigue 69, 1969.

21. C.Y. Kung, and M.E. Fine, "Fatigue Crack Initiation and Microcrack Growth in 2024-T4 and 2124-T4 Aluminum alloys", American Society for Metals and the 
Metallurgical Society of AIME, Metallurgical Transactions A, Volume 10A, May 1979. pp. 603-610.

22. J.C. Newman, Jr., and P.R. Edwards, "Short Crack Growth Behavior in an Aluminum Alloy", an AGARD Cooperative Test Program, AGARD Report AGARD-R-732, 1988.

23. J. C. Newman, Jr., E. P. Phillips, M. H. Swain, R. A. Everett, Jr., "Fracture Mechanics: An Assessment of a Unified Approach to Life Prediction," Advances in Fatigue Lifetime Predictive Techniques, ASTM STP 1122, Philadelphia, PA, USA, pg. 5-27, 1992.

24. R.J.H. Wanhill, "Short Cracks in Aerospace Structures," The Behaviour of Short Fatigue Cracks, EGF Pub 1 (Edited by K.J. Miller and E.R. de los Rios), 1986, Mechanical Engineering Publications, London, pp. 27-36.

25. N. M. Grinberg, V. A. Serdyuk, A. M. Gavrilyako, D. V. Lychagin, E. V. Kozlov, "Nucleation and Growth of Small Surface Cracks in Aluminium Alloy AMg6 as Related to Discontinuity of the Fatigue Curve," International Journal of Fatigue, Vol. 13, no. 5, pp. 370-376. Sept. 1991.

26. C. Kaynak, A. Ankara, "Short Fatigue Crack Growth in Al 2024-T3 and Al 7075T6," Engineering Fracture Mechanics. Vol. 43, no. 5, pg. 769-778. Nov. 1992.

27. M. H. Carvalho, M. Defreitas, "Short Crack Behaviour in Al-Li Alloy 2090," an AGARD Cooperative Test Program, AGARD Report AGARD-R-76, 1990.

28. Y. Murakami, S. Kodama, S. Konuma, "Quantitative Evaluation of Effects of Non-Metallic Inclusions on Fatigue Strength of High Strength Steels I: Basic Fatigue Mechanism and Evaluation of Correlation Between the Fatigue Fracture 
Stress and the Size and Location of Non-Metallic Inclusions," International Journal of Fatigue. Vol. 11, no. 5, pp. 291-298. Sept. 1989.

29. J. Lankford, "The Growth of Small Fatigue Cracks in 7075-T6 Aluminum," Fatigue of Engineering Materials and Structures, Volume 5, Issue 3, 1982, pp. 233-248.

30. A. Navarro, E. R. de los Rios, "A Model for Short Fatigue Crack Propagation With an Interpretation of the Short-Long Crack Transition," Fatigue and Fracture of Engineering Materials and Structures, Vol. 10, no. 2, pp. 169-186. 1987.

31. K. Hussain, E. R. de los Rios, "Application of Short and Long Fatigue Crack Growth Behavior in Lifetime Prediction of Mine Auxiliary Ventilation Fans," Materials Transactions, JIM. Vol. 35, no. 9, pp. 585-590. Sept. 1994.

32. P. Hyspecky, B. Strnadel, "Conversion of Short Fatigue Cracks Into a Long Crack," Fatigue and Fracture of Engineering Materials and Structures. Vol. 15, no. 9, pg. 845-854. Sept. 1992.

33. J. Goldstein, D. Newbury, P. Echlin, D. Joy, A.D. Romig, C. Lyman, C. Fiori, and E. Lifshin. Scanning Electron Microscopy and X-Ray Analysis, A Text for Biologists, Materials Scientists, and Geologists. Second Edition. Plenum Press, New York. 1992.

34. Standard Test Method for Measurement of Fatigue Crack Growth Rates, ASTM E $647-00$, ASTM, 2001.

35. J. Tong, "Notes on Direct Current Potential Drop Calibration for Crack Growth in Compact Tension Specimens," Journal of Testing and Evaluation, JTEVA, Vol. 29, No. 4, July 2001, pp. 402-406. 
36. ASM International Metals Handbook, Volume 2, $10^{\text {th }}$ Edition, Properties and Selection: Nonferrous Alloys and Special-Purpose Materials, pg. 3-177, 1990.

37. Aerospace Structural Metals Handbook, Nonferrous Alloys, Mechanical Properties Data Center, Department of Defence, Volume 3, 1991 Edition, code 3203, pg $1-15,1990$.

38. E. A. Starke, Jr., G. Lutjering, "Cyclic Plastic Deformation and Microstructure," Fatigue and Microstructure, St. Louis, Mo, pg. 205-243. 1979.

39. G. Lutjering and A. Gysler, "Fatigue and Fracture of Aluminum Alloys," Aluminum Transformation Technology and Applications, Proceeding of International Symposium at Puerto Madryn, Argentina (edited by C. A. Pampillo, H. Biloni, and D. E. Embury), ASM, Metals Park OH, pg 192, 1980. 
APPENDIX A - Image Pro Procedure 


\section{Image Pro Software Analysis Procedure}

The following is a step-by-step list of procedures for performing image analyses on the backscattered SEM micrographs taken during metallography and also during the postfracture analyses of the crack nucleation sites.

\section{Image Analysis}

\section{1) Open Picture in Image-Pro}
a) Click on "File"
b) Click on "Open"
c) Select picture file, click "Open"

\section{2) To Set up a New Spatial (i.e. New Calibration)}

* This step is only required if a new calibration is needed

a) Open the picture you wish to calibrate from. It is best to use one with a micro-marker.

b) Go to the "Measure" menu and click on "calibration"

c) Go to the "Spatial" button

d) Click "New"

e) Type in the Name for the new spatial and select the proper Units

f) Click on the "Image" button within the pixels/unit box, so that a calibration line appears on the image. 
g) Drag and stretch the line to a known distance. Zoom in for more accuracy with magnifying glass on tool bar. Hold the shift button down to zoom out.

h) Enter the true length of the line in the scaling box and click " $O K$ "

\section{3) To Select an Existing Spatial}

* This step is only required when first opening program or for new set of pictures taken at a different magnification.

a) Go to the "Measure" menu and click on "calibration"

b) Click on the "Select Spatial" button

c) Select the desired spatial and click " $O K$ "

\section{4) Area of Interest (AOI)}

* Only use if there is a specific area on the picture you want to focus on.

a) Select one of the AOI tools (rectangular, ellipse, irregular, or multiple) from the tool bar

b) Click and drag to select the area of interest

c) Go to the "Edit" menu and click on "Duplicate/Crop to AOr' button to create a new image with only the area of interest

\section{5) Adjusting Contrast}

* This may be necessary for pictures which are too dark and the details are difficult to see

a) Under "Enhance", choose "Contrast Enhancement". 
b) Adjust brightness and contrast knobs to optimize the photograph

c) Click "Apply"

d) Click " $O K$ "

e) Close contrast enhancement window

\section{6) Measuring}

a) Open picture file to measure. Use one that does not have a micro-marker.

b) Go to the "Measure" menu and click on "Count/Size"

c) Make sure these options are selected:

- Manual:

Measure Objects

$\checkmark$ Apply Filter Ranges

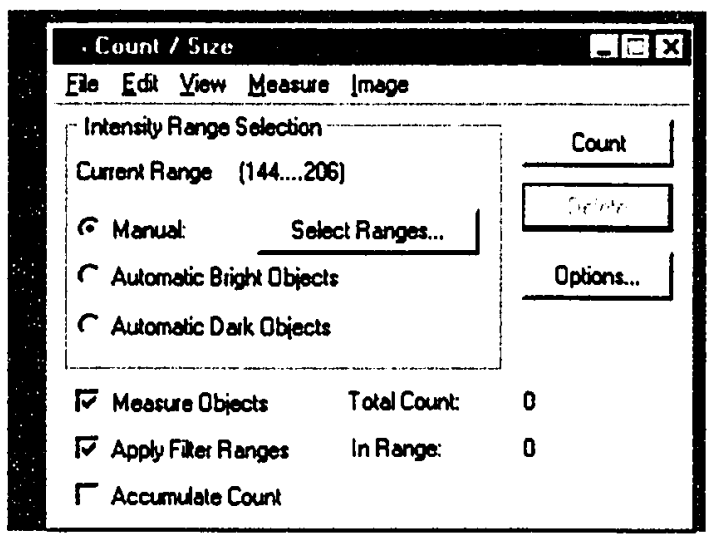

d) Under "Options" in the "Count/Size" box select 8-connect in object options and click "OK".

e) Inside the "Count/Size" menu, go to the "Measure" menu 
f) Click on the "Select Measurements" button and Select the following measurements with the following ranges:

- Angle

- Area

- Aspect

- Axis (Major)

- Axis (Minor)

- Box Height

- Box Width

- $B o x X / Y$

- Center X

- Center $Y$

- Class

- Cluster

- Diameter (Max)

- Diameter (Mean)

- Diameter (Min)

- Per-Area

- Perimeter

- Perimeter (Ellipse)

- Radius (Max)

- Radius (Min)

- Radius Ratio

$$
0-180
$$

$0.50-\max$

$0-\max$

$0-\max$

$0-\max$

$0-\max$

$0-\max$

$0-\max$

$0-\max$

$0-\max$

$0-16$

$0-\max$

$0-\max$

$0-\max$

$0-\max$

$0-1$

$0-\max$

$0-\max$

$0-\max$

$0-\max$

$0-\max$ 
- Roundness

$$
0-\max
$$

- Size (length)

$$
0-\max
$$

- Size (width)

$0-\max$

* Max is about $10^{8}$. This value was chosen to make sure all upper data is included.

g) Click " $O K$ "

h) To save these settings, go to "File" in "Count/Size" and click on "Save Settings." These settings will stay until the program is shut down. For the measurement on the next picture, all of the above steps do not need to be performed again. When opening the program the next time, these saved settings can be retrieved by going to "File" in "Count/Size", and clicking on “Load Settings".

i) Click on the "Select Ranges" button. A histogram-based box will appear with peaks and valleys for different concentrations of the gray scale. To get a more accurate view click down on the bar to the right of the graph.

j) Select the Class 1 colored line at the top of the graph. Click and drag the upper range line so that it covers the matrix (large peak). Leave some space open on the right end of the graph. Do not leave any space open on the left side. 


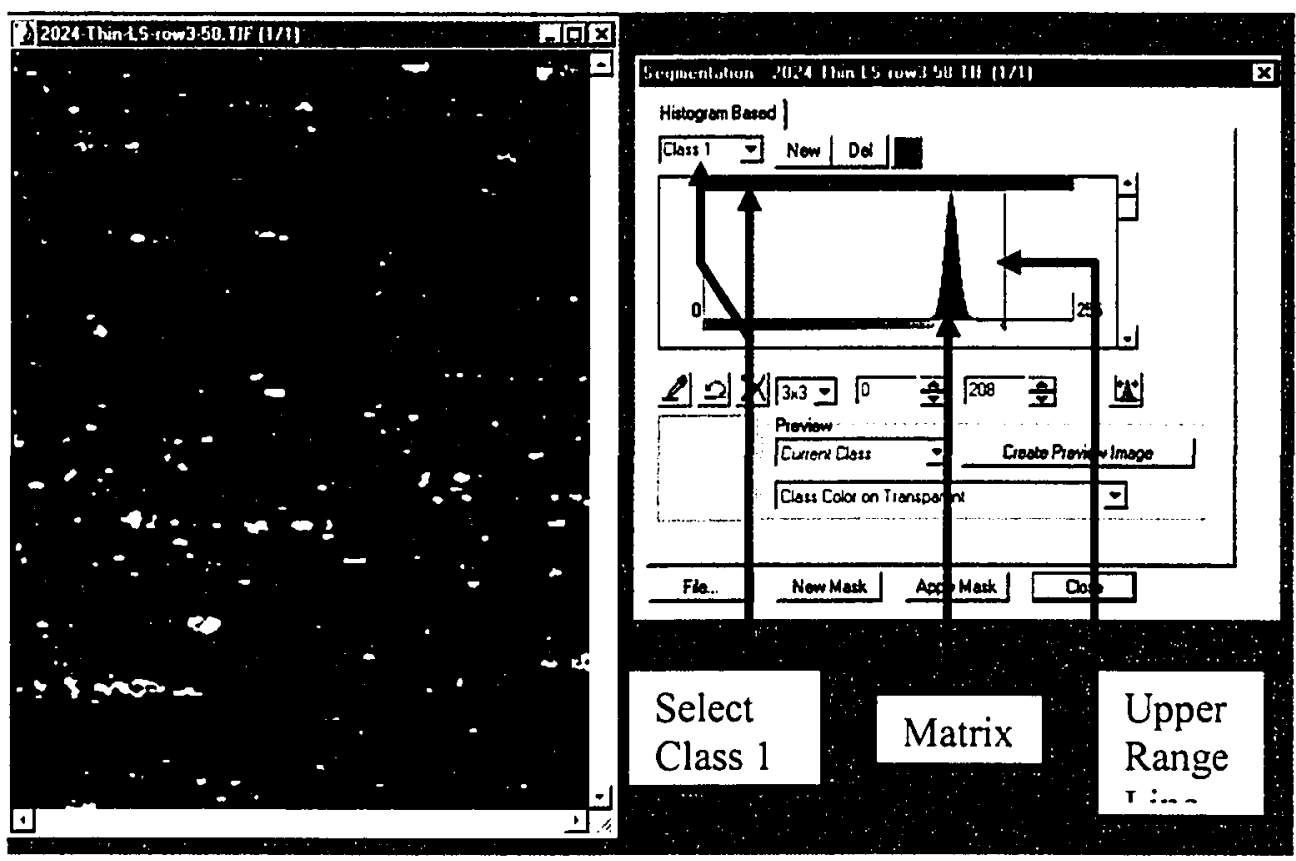

k) Click on "New" to create another class for the white particles. A new color bar will appear on the right end of the graph.

1) Adjust the two classes by selecting the color bars and dragging the upper and lower ranges so that the new class covers ONLY and ALL of the white particles and goes to the far right of the graph. 


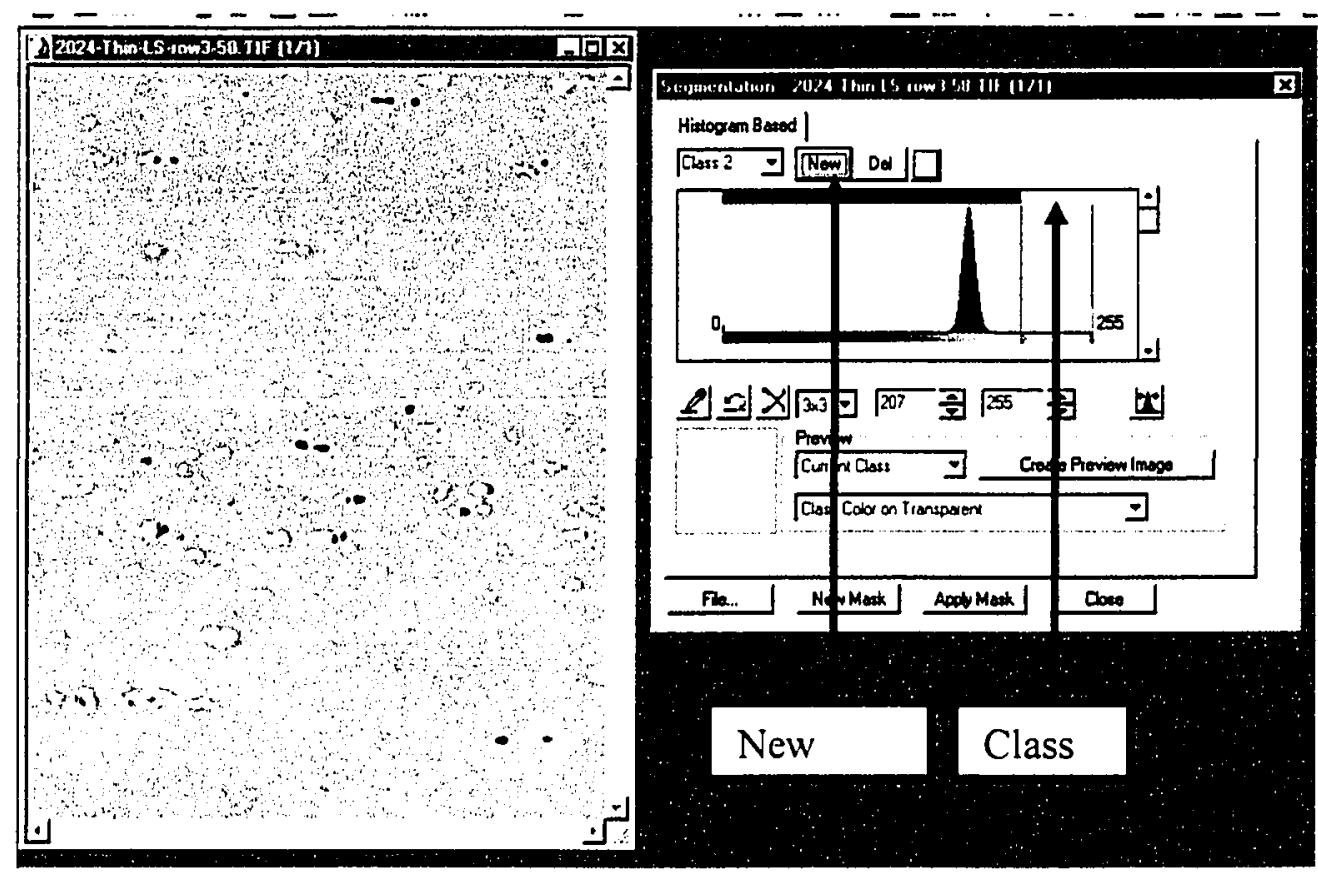

m) Click and drag the lower limit of the first class to cover only the matrix, leaving room on the left of the graph.

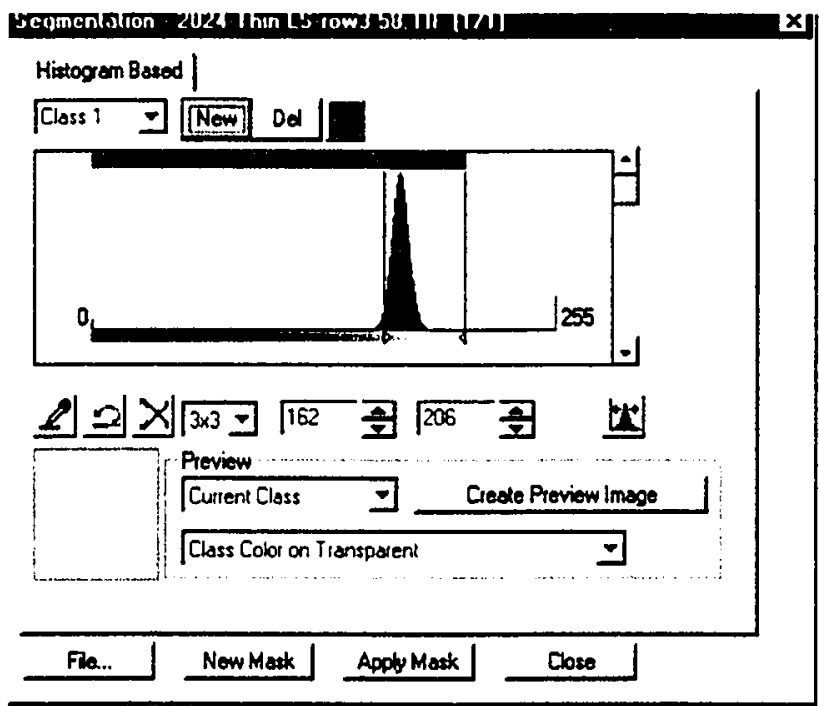

n) Click on "New" to create another class for the black particles 
o) Ensure that the new class covers ONLY and ALL of the black particles

p) The three classes should now cover the whole graph.

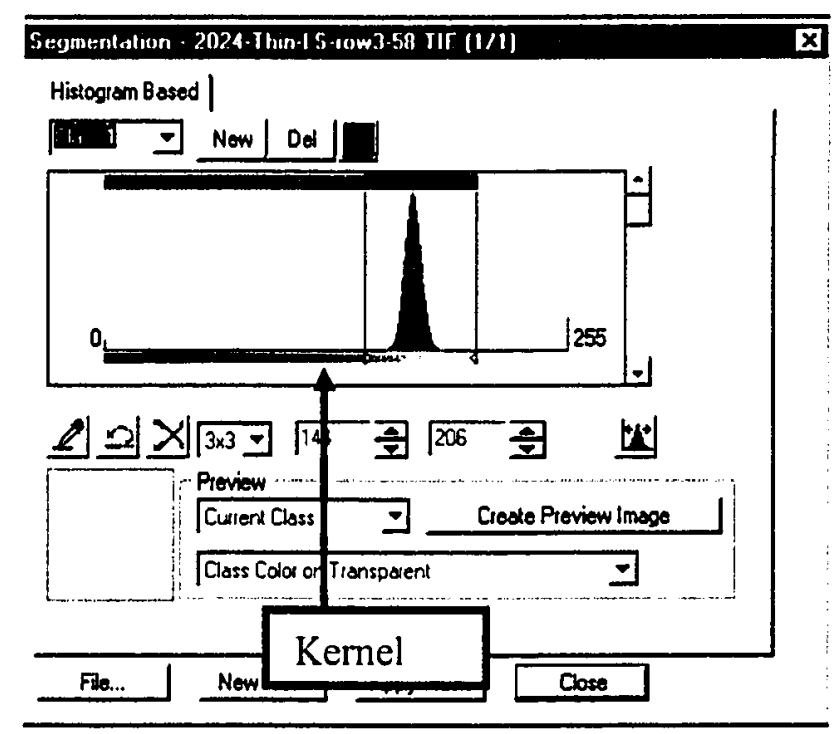

q) Change the kernel size (box right below graph) to $1 \times 1$ rather than $3 \times 3$

r) Click on the "Close" button

s) Click on the "Count" button 


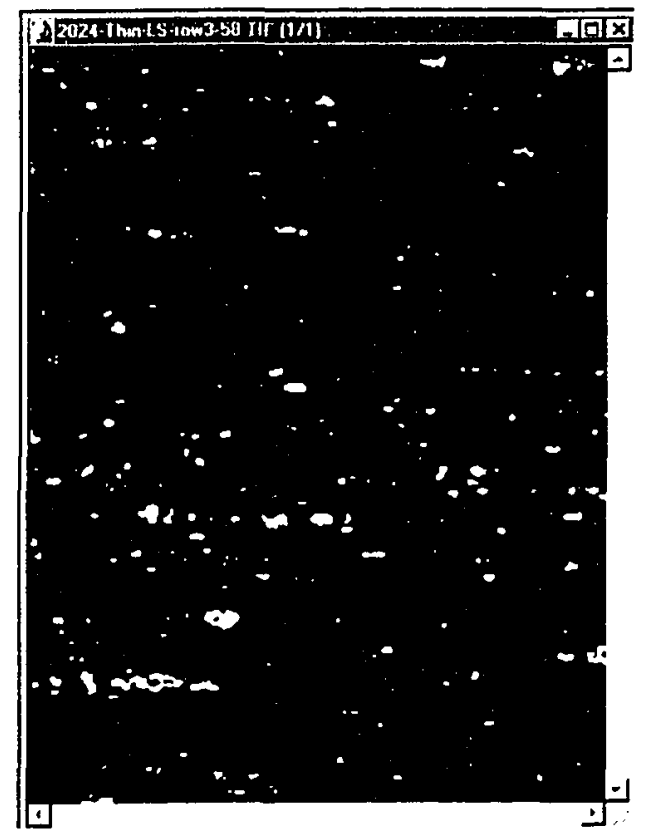

\section{Results and Analysis}

1) In the Count/Size window, click on the "View" menu and select "Measurement Data"

2) In the Count/Size window, click on the "View" menu and select "Range Statistics"

3) In order to export these data to excel:

a) Open a new worksheet in Excel

b) In each window("Measurement Data" and "Range Statistics"), go to the "File" menu

c) Click on "DDE to Excel" (The data will appear in an Excel worksheet)

d) Put "Measurement Data" on Sheet 1 and "Range Statistics" on Sheet 2. 
* Make sure sheet 2 is selected before transferring the "Range Statistics" or else it will copy over top the measurements on sheet 1.

e) Save and close the worksheet

f) Repeat for the other results

* If the worksheet is not closed before exporting another set of data, the new set of data will be copied over top of the older data.

\section{Making a Collage of Large Particles}

1) Select all of the micrographs whose particles will be collected into the collage.

2) Follow steps one through five of the Image Analvsis section.

3) Click on the "Select Measurements" button and set the area minimum to a value of 5 or $10 \mu \mathrm{m}^{2}$ to capture only the large particles.

4) Click " $O K$ "

5) Follow step six of the Image Analvsis section. Only cover particles and holes by ranges. Do not cover the matrix.

6) Click on the "Image" button within the Count/Size window. Then Select "Make Sorted Image"

7) Select the sort by "Area" option and leave the box for "Display Objects Vertically" unchecked. Set the background to "Automatic" and click "Sort Objects". 


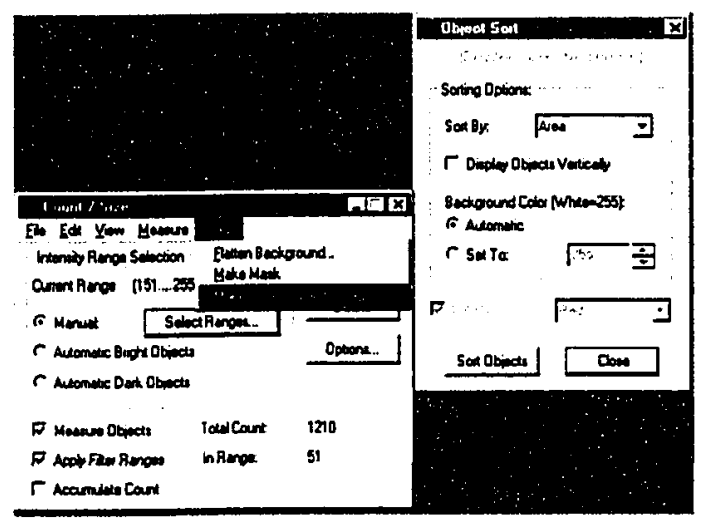

8) A new picture of the particles (and holes) sorted from largest to smallest will appear.

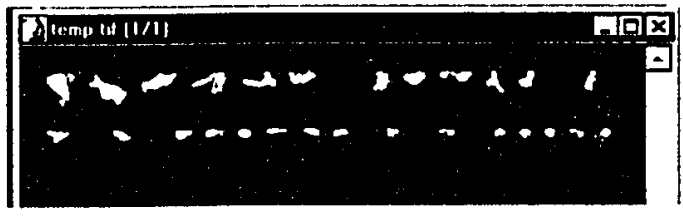

9) Repeat steps 3 through 9 for the remaining micrographs.

10) To make one larger file for a collection of pictures draw an AOI around a set of particles and then cut (or copy) and paste on to one picture.

11) Repeat steps 3 through 9 to sort the particles in the correct order. The limits can be changed in step 4 if it is required (in case where there are too many particles). 


\section{Adding Fracture Particles to Collage}

1) Select the best (Back-Scattered) SEM photo of fracture particle

2) Select the appropriate spatial or create new spatial, as described in the Image Analysis section.

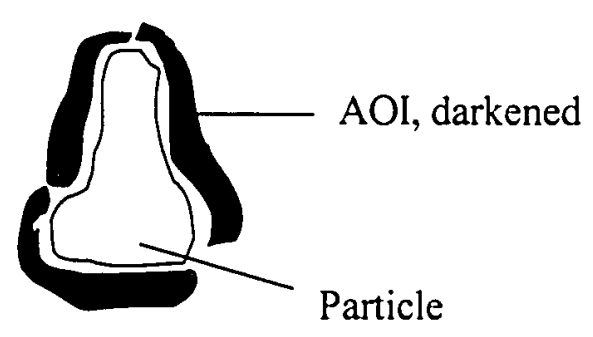

3) Define an irregular (free hand) area of interest (AOI) around particle and decrease contrast and brightness to darken the area surrounding particle. This will ensure that only the particle will be detected during the analysis.

4) Select a new AOI (square or otherwise) surrounding the particle. Make sure the micro-marker is outside of the AOI. 


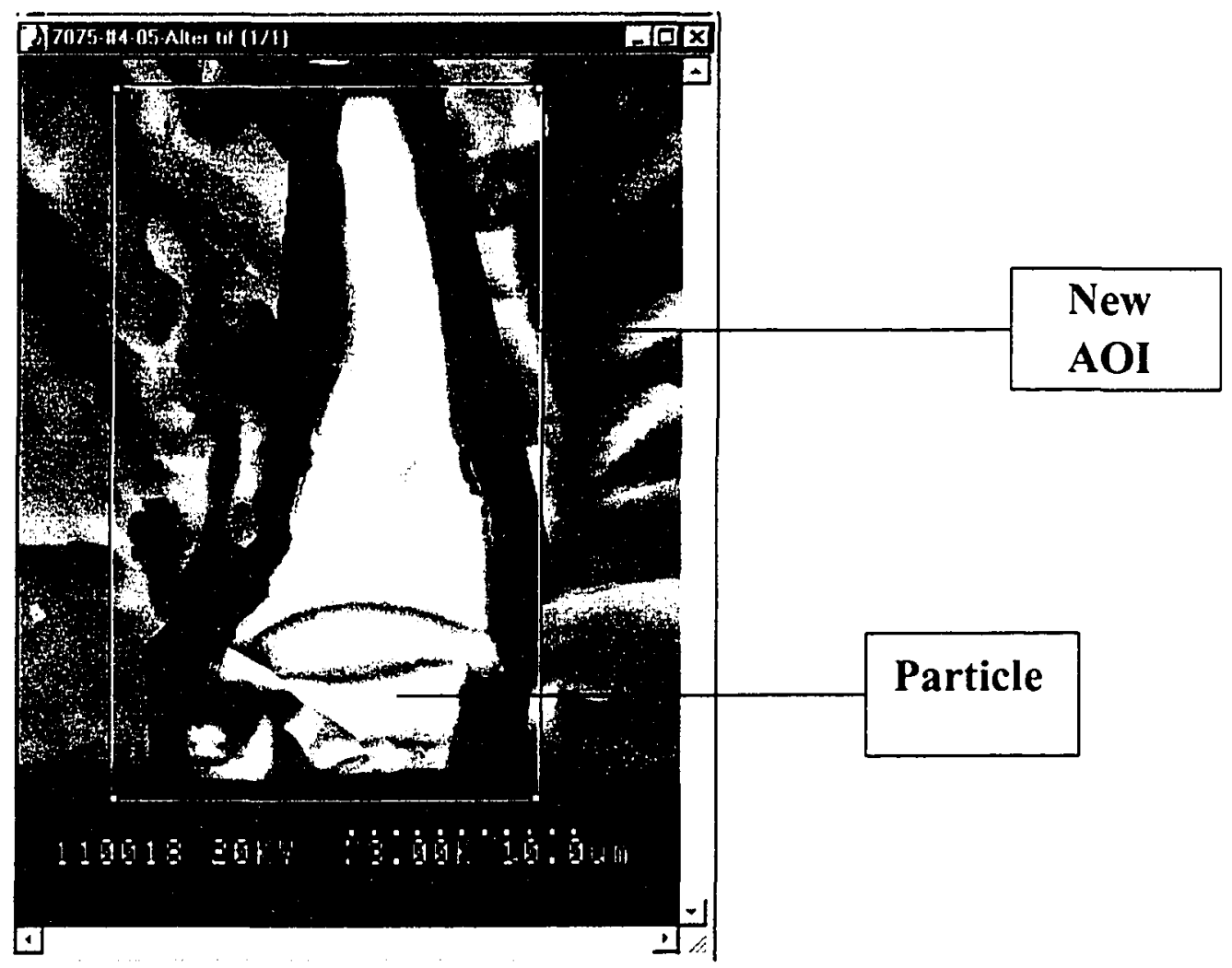

5) Perform step six of the Image Analysis section.

6) Click on the "Image" button within the CountSize window. Then Select "Make Sorted Image"

7) Select sort by "Area" and leave the box for "Display Objects Vertically" Unchecked. Set the background to "Automatic". Click "Sort Objects".

8) If the fracture particle is from the transverse side of the fatigue specimen, the image can be rotated $90^{\circ}$ so it is comparable to the specimens that broke from the short transverse side. Select the picture, click "Edit", "Rotate", "Rotate image $90^{\circ}$ " and then "Maintain Image Size".

a) Select the sorted image and go to the "Measure" menu and click on "calibration"

b) Click on the "Select Spatial' button. 
c) Calibrate the picture in the same spatial as the original micrograph where the particle was taken from. Select the desired spatial and click "OK".

d) It will be required to measure the height of the particle in the original micrograph and the height of the particle in the new rotated image. Select "Measure" from the main menu, then "Measurements", and use the length feature to determine the height of the particle. Measure the same spot for both pictures.

e) Perform the following calculation to determine the amount the new picture needs to be resized.

f) Select the new sorted picture, click "Edit" from the main menu and select "Resize".

g) Enter the percent determined from the resizing formula, and click " $O K$ ".

h) Check that the resized picture is the same size as the original fracture surface picture (in its original spatial). 
APPENDIX B - Microsoft Excel Analysis Procedure 


\section{Excel Analysis Procedure}

The following is a step-by-step list of procedures for performing a statistical analysis

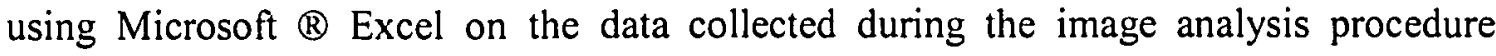
mentioned above.

\section{XY, Coordinate Plots}

The following procedures should be used to display the co-ordinates of all the particles analyzed during the metallographic analysis. This is performed to observe if there are any patterns in regards to the distribution of larger particles through the thickness.

1. Firstly, the Center-X and Center-Y columns in the ROW files need to be adjusted. Start with the first Row file (row 1), and insert a column right after Center-X and one after Center-Y. Call these columns Mod X and Mod Y, respectively. These columns will hold the adjusted values for plotting the particles.

2. The micrographs, which are taken at $500 \mathrm{X}$, have the dimensions shown below. Make sure that this is the same size as the micrograph being analyzed. The Center- $\mathrm{X}$ and $\mathrm{Y}$ columns need to be adjusted because the coordinates in the excel file assume that the origin is in the top left hand corner of every picture. This needs to be changed so that the origin is in the top right hand corner of the only first picture, and offset the others by the right number of picture heights or thickness'. For the first row, nothing needs to be done to the $\mathrm{Y}$ column. However, the $\mathrm{X}$ column needs to be fixed and it will always be altered this way for all of the rows. The first picture in this row does not 
need to be modified, therefore, fill the Mod X column with the same data as the center-X column. For the next picture, add the value from the Center-X column with the width of the picture $(177.78 \mu \mathrm{m})$, and put this value in the mod-X column. For the third picture, add the value of the center-X column to two widths of the picture ( 2 $\mathrm{X} 177.78 \mu \mathrm{m}=355.56 \mu \mathrm{m})$. Continue the same pattern by increasing the number of widths added to each of the following pictures in that row. For the $2^{\text {nd }}$ row, the $X$ coordinates will be determined the same way as above. The Mod-Y column for this row will be determined by adding the Center-Y data to one height of the picture $(228.806 \mu \mathrm{m})$. For Row 3, the $\mathrm{Y}$-coordinates will be determined by adding the center$\mathrm{Y}$ data to two of the picture heights $(2 \mathrm{X} 228.806 \mu \mathrm{m}=457.612 \mu \mathrm{m})$. The Mod-X Mod-Y data both continue with this pattern. Once all of the files in a row have been modified, copy all of that data into a new excel spreadsheet. Repeat so that a file is created for every row analyzed.

\begin{tabular}{|l|r|}
\hline $\mathrm{X}(\mu \mathrm{m})$ & 177.76 \\
\hline $\mathrm{Y}(\mu \mathrm{m})$ & 228.806 \\
\hline
\end{tabular}

3. Copy and paste all the data from the row files into one new file for the entire plane. It is not important to label the rows or picture numbers for this spreadsheet.

4. Highlight all of the data and sort the area by ascending order.

5. Now the coordinate map can be plotted for this sample. Create a scatter graph with the Mod-X column as the $X$ values and Mod- $Y$ column as the $Y$ values. Add gridlines to represent the size of the photos. Gridlines on the $\mathrm{X}$-axis should have a major unit of 177.76 , and the $Y$-axis should have a major unit of 228.8 . 
6. New plots can be made with different lower area limits (i.e. $10,25,50,75$, or 100 $\mu \mathrm{m}^{2}$ ) to determine if any patterns exist. Scroll down the list of sorted area data and plot only the particles that are of interest in the same manner as described in step five.

\section{Example Plot of Particles over $100 \mu \mathrm{m}^{2}$}

Grid lines used to represent one Dicture.

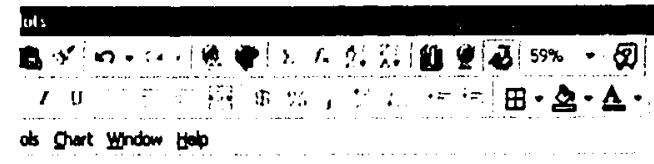

at Ghert Whindow that

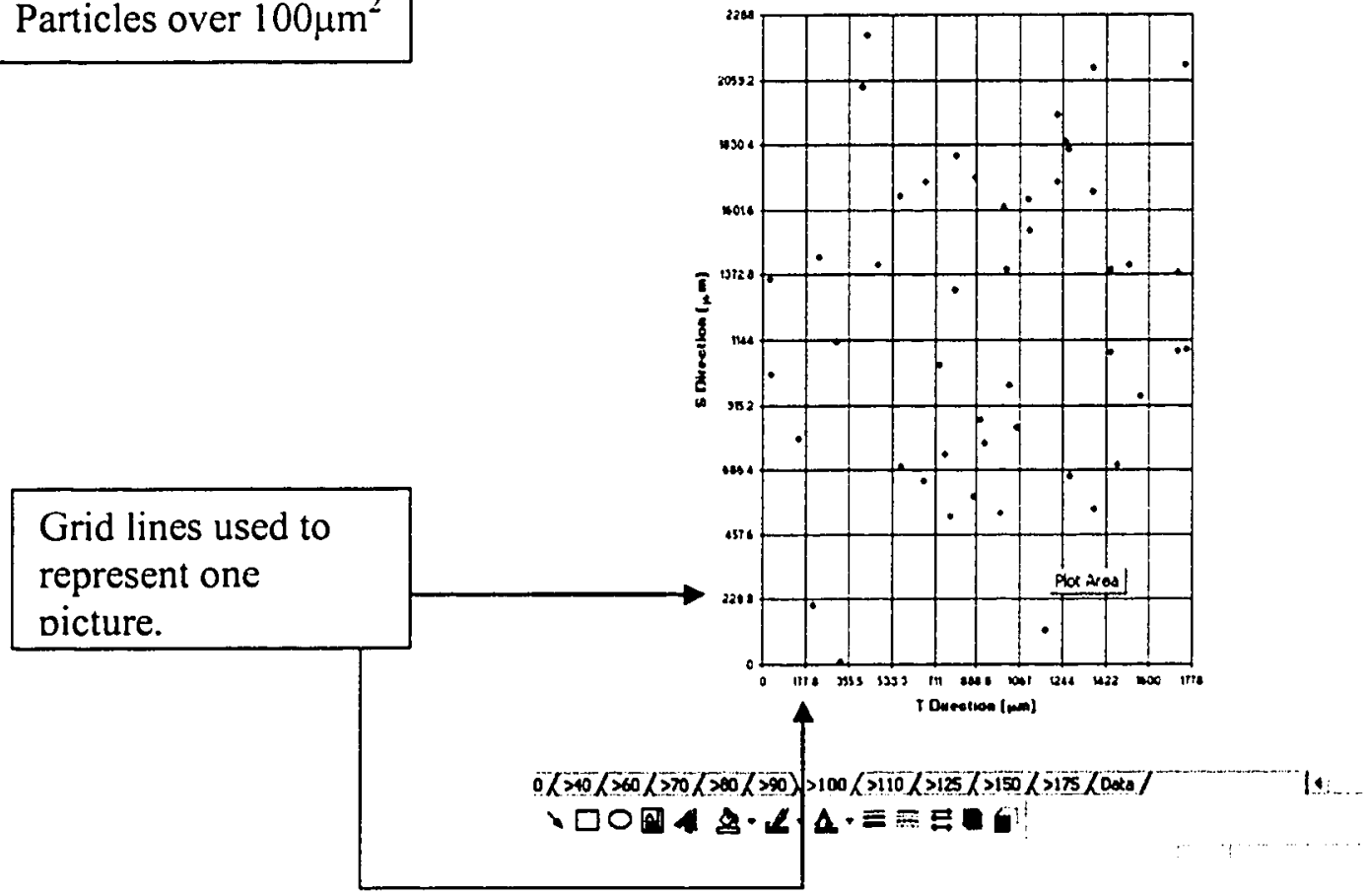




\section{Row Comparison}

The row comparison is performed as a simple check on the pattern of particles sizes and shapes throughout the thickness of a plane.

1. From each row file, collect an average value for each of the column headings.

2. Put this data together in a new spreadsheet as shown below.

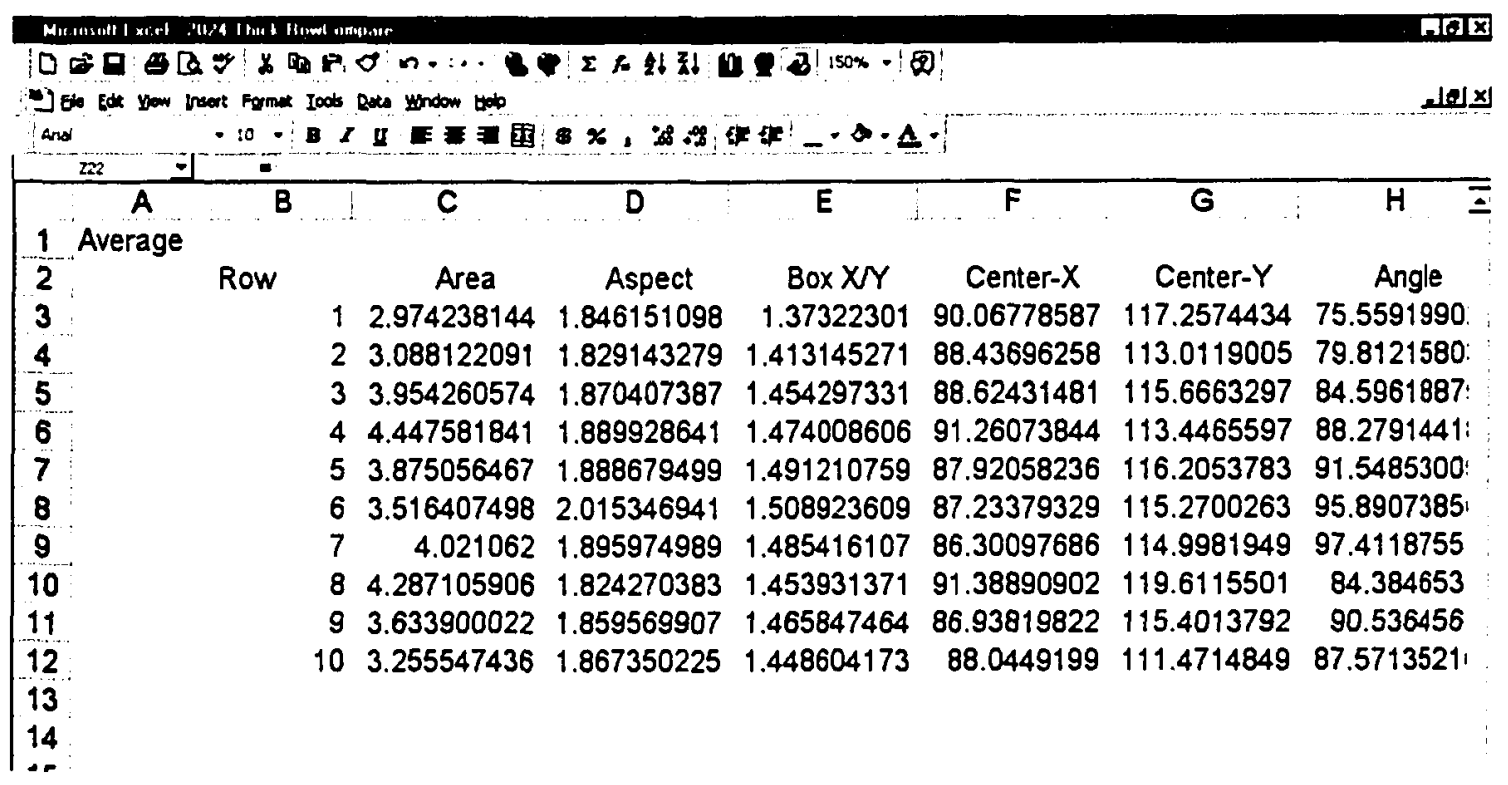

3. Plot the average values of area, width, height, box aspect ratio, and area percentage on a graph with the row number on the $\mathrm{x}$-axis. Use a scatter plot with smooth connecting lines. 


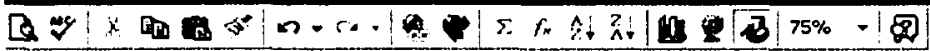

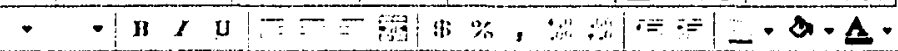

Insert Formak Iooks chart window telp

$=1$
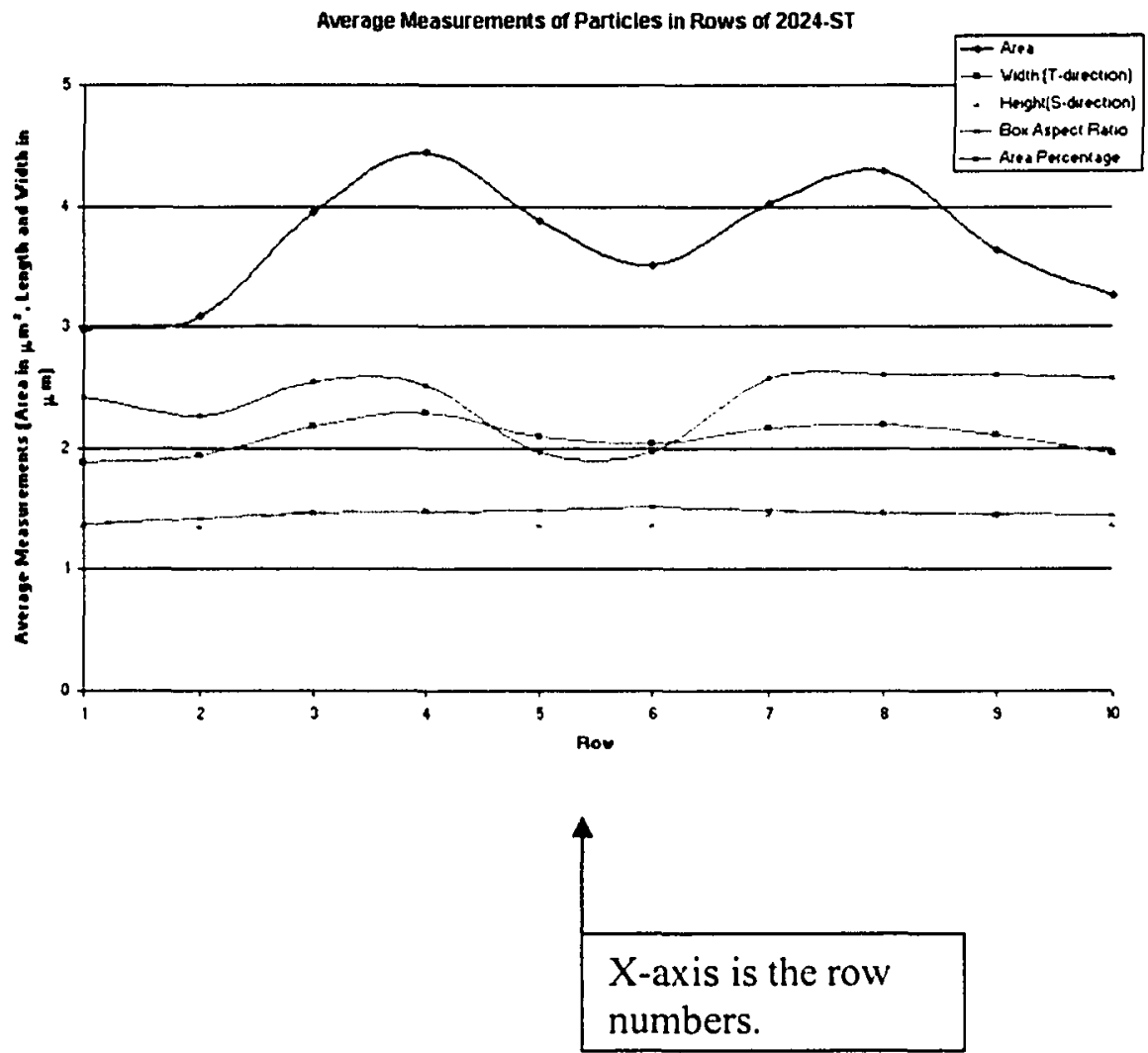


\section{Histograms}

By sorting data into groups of numbers called bins, the distribution curve can be plotted.

Histograms are being used to plot areas and dimensions of particles.

1. Click "Tools" on the main menu, then "Data Analysis" and choose "Histogram" from the menu. Click "OK".

2. In the "Input Range" box, insert the cells of the area, or another dimension that is of interest.

3. In the "Bin Range" box, insert the cells of the divisions to be plotted. Have a column set up for this with even increments. For example, $0.25 \mu \mathrm{m}^{2}$ was used as a division, and the increments started at $0.75 \mu \mathrm{m}^{2}$. The first bin of $0.75 \mu \mathrm{m}^{2}$ will count the number of particles with areas smaller than $0.75 \mu \mathrm{m}^{2}$. Since the area data starts at 0.5 $\mu \mathrm{m}^{2}$, the increment is still $0.25 \mu \mathrm{m}^{2}$ for this first bin.
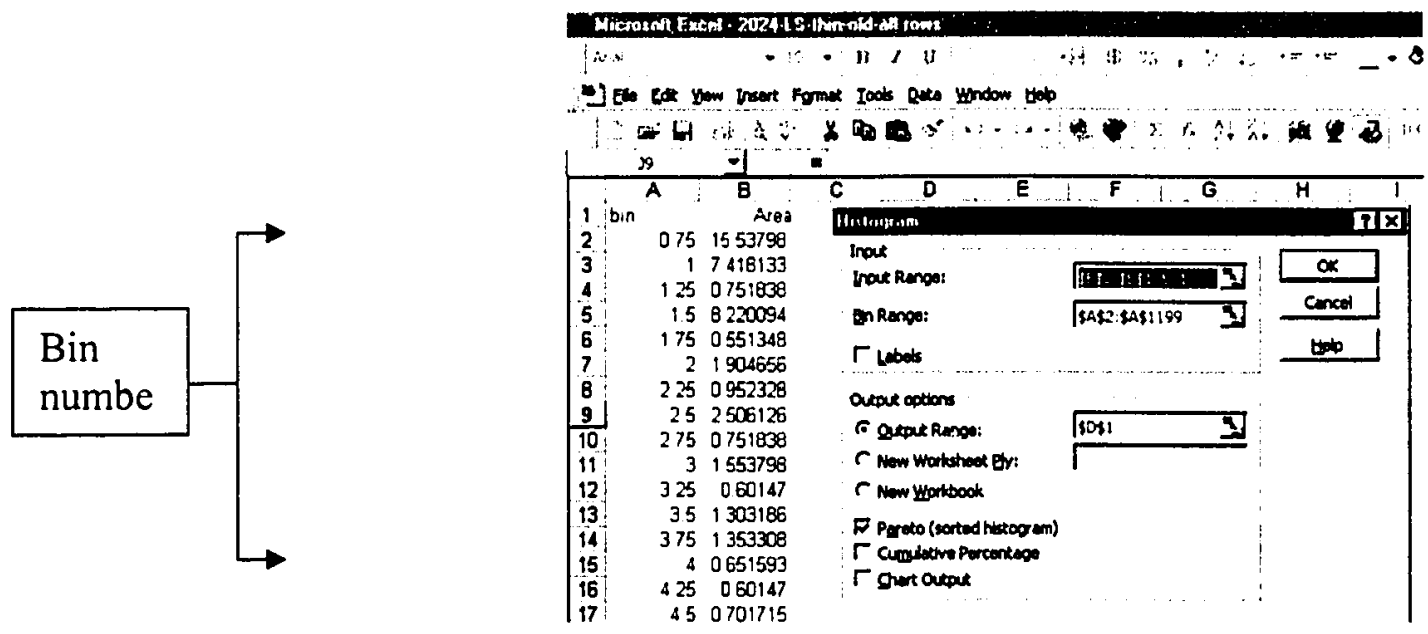
4. Select the output range to be somewhere on the same worksheet.

5. Choose a "Pareto (sorted histogram)". Click "OK"

\begin{tabular}{|c|c|c|c|c|}
\hline \multicolumn{5}{|l|}{ 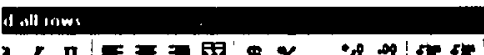 } \\
\hline \multicolumn{5}{|c|}{ oots Roke Yundow tob } \\
\hline \multicolumn{5}{|c|}{$\Delta=\varnothing \cdot \cdots$} \\
\hline D & $\ldots E$ & $F$ & $G$ & $\mathrm{H}$ \\
\hline En & Frequency & an & Froquency & fraction \\
\hline 075 & $4 \quad 4845$ & 075 & 4845 & 0276857 \\
\hline$i$ & 2663 & 1 & 2663 & 0152171 \\
\hline 125 & 1650 & 125 & 1650 & 0094266 \\
\hline 15 & 1068 & 15 & 1068 & 0051029 \\
\hline 1.75 & 763 & 175 & 763 & 00436 \\
\hline ? & 5.44 & 2 & 544 & 0,0310006 \\
\hline 225 & 461 & 225 & $46:$ & 0026343 \\
\hline 25 & 344 & 25 & 344 & 0049657 \\
\hline 275 & 255 & 3 & 265 & 0015143 \\
\hline 3 & 265 & 275 & 255 & 0014571 \\
\hline 3.25 & 238 & 325 & 238 & 00136 \\
\hline 35 & 198 & 35 & 198 & $00113: 4$ \\
\hline 375 & 188 & 375 & 188 & 0010743 \\
\hline 4 & 173 & 4 & 173 & 0,008868 \\
\hline 4.25 & 146 & 4.25 & 146 & 0,008343 \\
\hline 45 & 125 & 55 & 130 & $0,0074 \times 9$ \\
\hline 475 & 126 & 475 & 126 & 00072 \\
\hline 5 & 118 & 45 & 125 & 0.007143 \\
\hline$=x$ & in & $c$ & 110 & nimsers \\
\hline
\end{tabular}

6. Four columns will appear. The first two are the bin and frequency and the second two are the same but sorted by frequency in descending order. Delete the bottom of the last two columns that have zero frequency.

7. Create a column called "Fraction" beside the sorted frequency column. The values will be calculated from dividing the corresponding frequency value by the total number of particles.

8. With the $x$-axis as the bin numbers and $y$-axis as the fraction column, a log scale plot should be created, as shown below. 


\section{Area Distribution of Particles in LS Plane of Old Thin 2024}

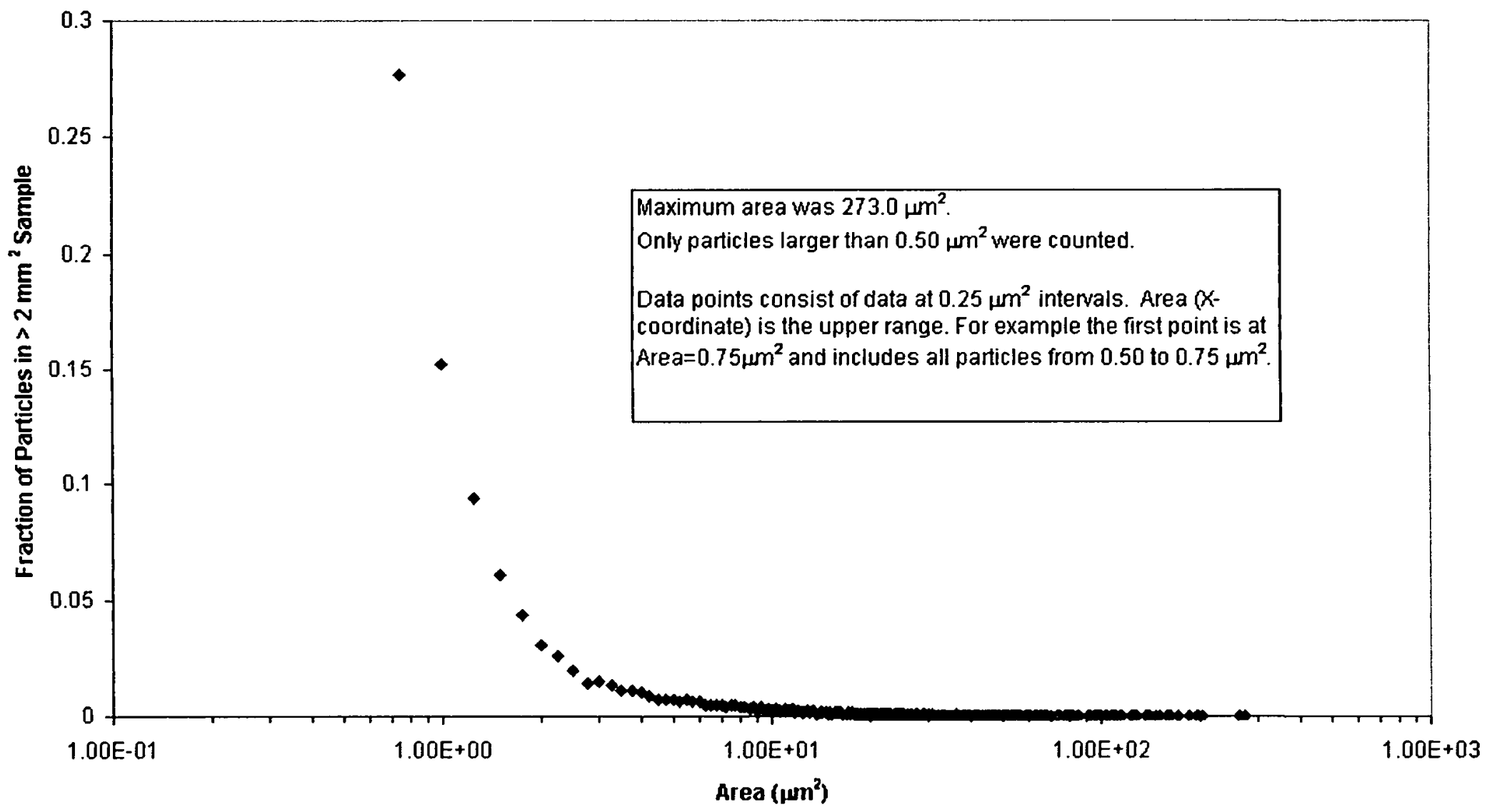




\section{Weibull Excel Procedure}

Weibull is useful in describing the distribution of particle sizes in this thesis work.

1) Collect all data for a characteristic dimension (area, length, width etc.) of the particles from one plane of an alloy.

2) Sort the data in ascending order. This column will be referred to as " $t$ ".

3) For sets of numbers that do not begin at zero, a correction must be applied. This is used because the particles whose area is less than $0.50 \mu \mathrm{m}^{2}$, have been truncated. So the $t$ column is adjusted by subtracting 0.5 (or the lowest value).

4) Find total number of particles in the sample examined.

5) Assign a position number, " $i$ ", to each particle area (t). 1 for the smallest particle area and the total number of particles corresponding to the largest area.

6) Determine an approximate $F$ for each $t$ value. $F$ is the position number less 0.5 and then divided by the total number of particles $(\mathrm{N}) . \mathrm{F}=(\mathrm{i}-0.5) / \mathrm{N}$

7) The values for the $x$ and $y$-axis are $\ln (t)$, and $\ln (\ln (1 /(1-F)))$, respectively. Set up two columns that calculate these values.

8) Plot the $x$ and $y$-axis values. The plot must be linear to fit weibull distribution.

9) Add a trendline to the plot and choose linear regression. If the start or end of the graph is not linear then only fit the line to the linear portion of graph.

10) In the trendline window, enable the option that will show the equation of the plot on the graph. 
11) Using this equation which is in the form of $y=m x+b$, find $\alpha$ and $\beta$, where $\alpha=e^{b}$, and $\beta=\mathrm{m}$.

12) In the excel spreadsheet, use the formula "=WEIBULL" and the $\alpha$ and $\beta$ values to determine the Weibull distribution for every t. Four parameters are required to calculate the Weibull values.

$\mathbf{X}$, which will be the $t$ value at which to evaluate the function (not the adjusted $t$ value)

Alpha is $\alpha\left(=e^{b}\right)$

Beta is $\beta(=m)$

Cumulative a) True will return values for a cumulative distribution function (true is chosen for a Weibull distribution).

b) False will return values for a percent mass function.

13) Plot the new weibull values against their corresponding $t$ values (all $t$ values).

Shown below is an example of the steps above. 


\section{Weibull Distribution of Area}

\section{Example from 2024-ST Plane, 0.063" thickness}

\begin{tabular}{|c|c|c|c|c|c|c|}
\hline 1) Area & 2) Size (Area) & 3) Adjusted Area & 5) $i$ & 6) $F$ & 7) $x$ & 7) $Y$ \\
\hline 2.5061262 & 0.50122523 & 0 & 1 & $4.17049 E-05$ & \#NUM! & -10.08487117 \\
\hline 1.8545333 & 0.50122523 & 0 & 2 & 0.000125115 & \#NUM! & -8.986217173 \\
\hline 1.5036757 & 0.50122523 & 0 & 3 & 0.000208524 & \#NUM! & -8.475349839 \\
\hline 0.65159279 & 0.50122523 & 0 & 4 & 0.000291934 & \#NUM! & -8.138835888 \\
\hline 2.6063712 & 0.50122523 & 0 & 5 & 0.000375344 & \#NUM! & -7.887479744 \\
\hline : & : & $\cdot$ & : & : & $:$ & : \\
\hline : & : & : & : & : & : & : \\
\hline 1.6039207 & 211.56717 & 211.0659448 & 11985 & 0.999624656 & 5.352170619 & 2.065300457 \\
\hline 5.3631101 & 225.15038 & 224.6491548 & 11986 & 0.999708066 & 5.414539873 & 2.096665095 \\
\hline 2.0550234 & 245.80084 & 245.2996148 & 11987 & 0.999791476 & 5.502480381 & 2.137174234 \\
\hline 0.75183785 & 248.20673 & 247.7055048 & 11988 & 0.999874885 & 5.51224056 & 2.19569894 \\
\hline 2.9071062 & 581.22076 & 580.7195348 & 11989 & 0.999958295 & 6.364267912 & 2.311038465 \\
\hline
\end{tabular}

12)

\begin{tabular}{l|r|} 
n & -0.6318 \\
\cline { 2 - 2 } & 0.6531 \\
\cline { 2 - 2 } & 0.531633998 \\
\hline
\end{tabular}

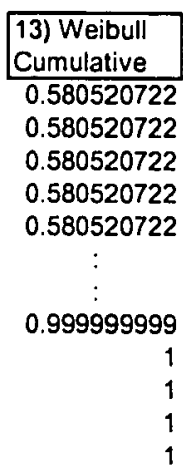




\section{Weibull Distribution of Area of Particles in ST Plane of 2024-Thin}

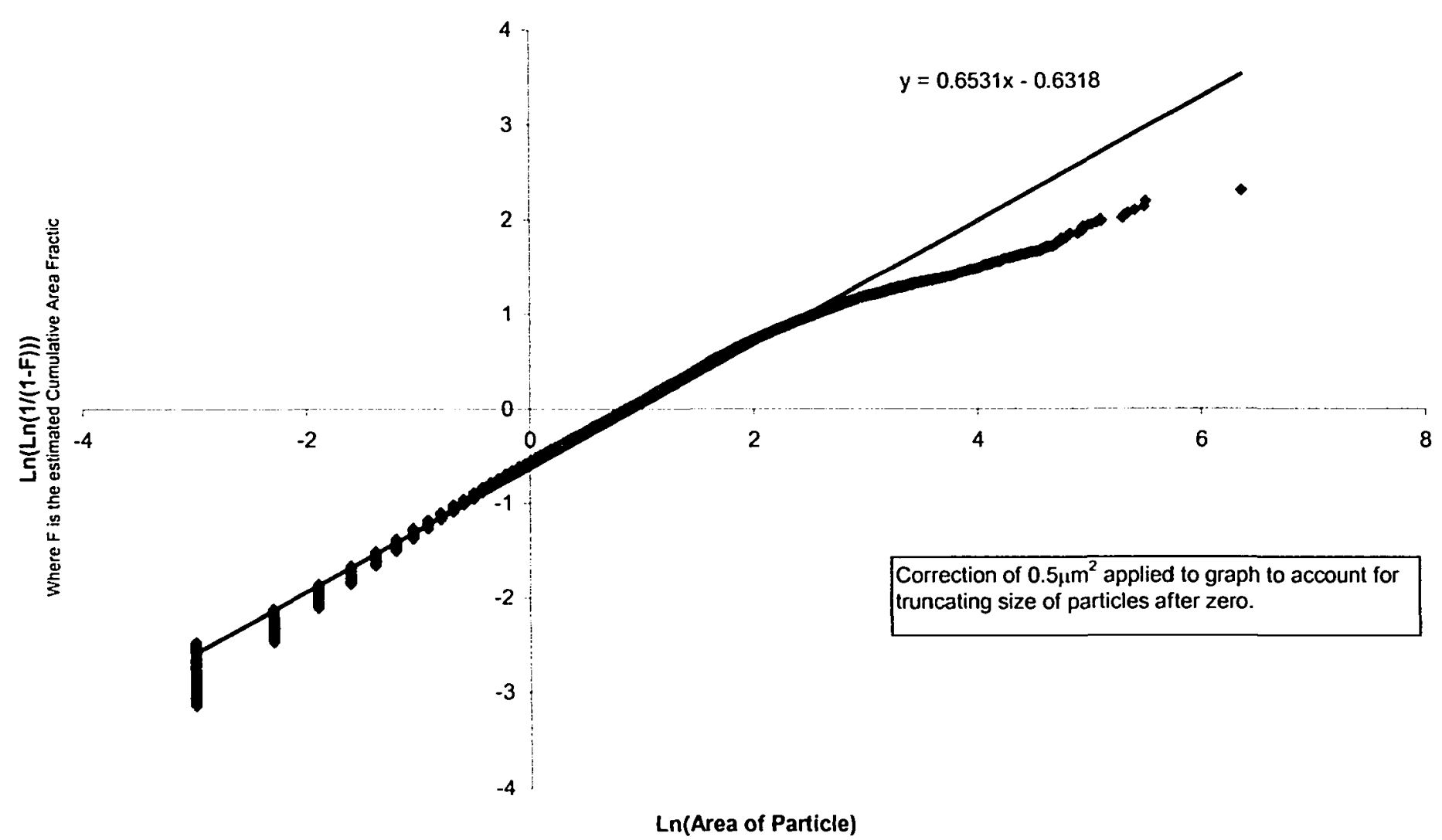

7) Weibull XY plot of 2024-ST Plane ,0.063" thickness 
Cumulative Weibull Distribution of Area of Particles in ST Plane of 2024-Thin

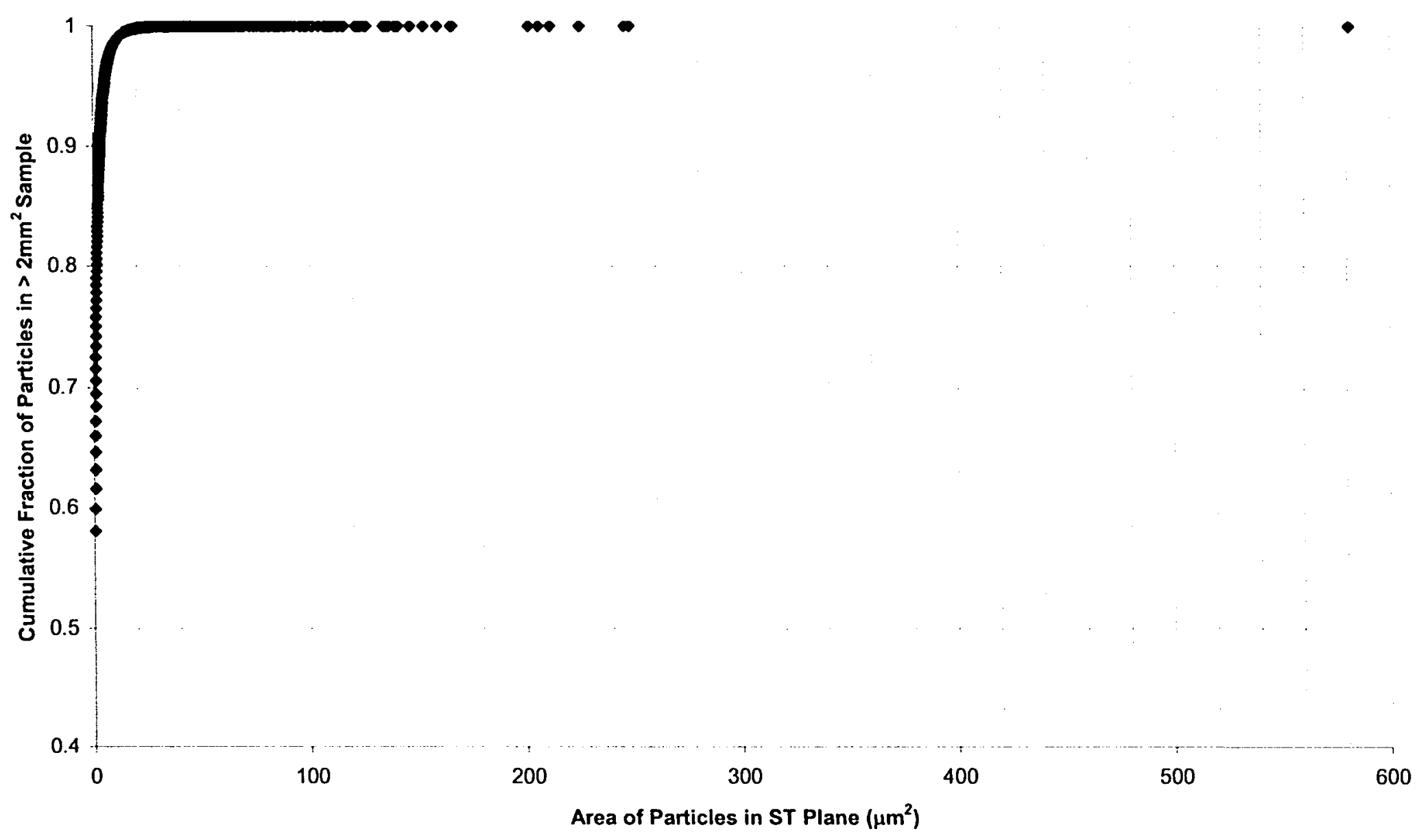

13) Cumulative Weibull Plot 


\section{APPENDIX C - Specimen Preparation and Loading Procedure}




\section{Procedure for Loading Single-edge Notched (SEN) Fatigue Specimens for Nucleation}

\section{and Short Crack Study}

1. Polish the interior of the notch with 600,800 , and 1200 grit polishing paper, leaving a nice finish in order to eliminate any fluctuations in fatigue performance due to machining marks.

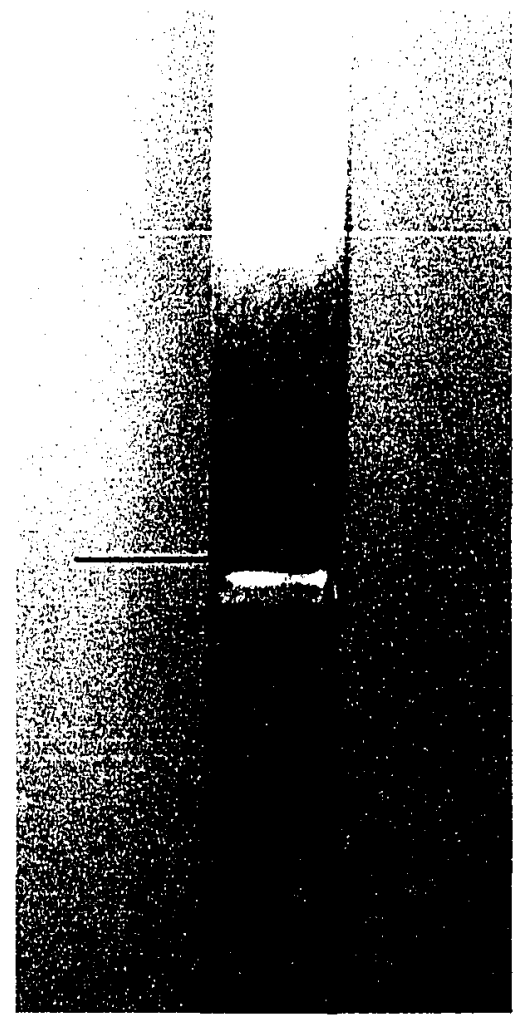


2. Break the edges surrounding the notch area to eliminate areas of stress concentration.

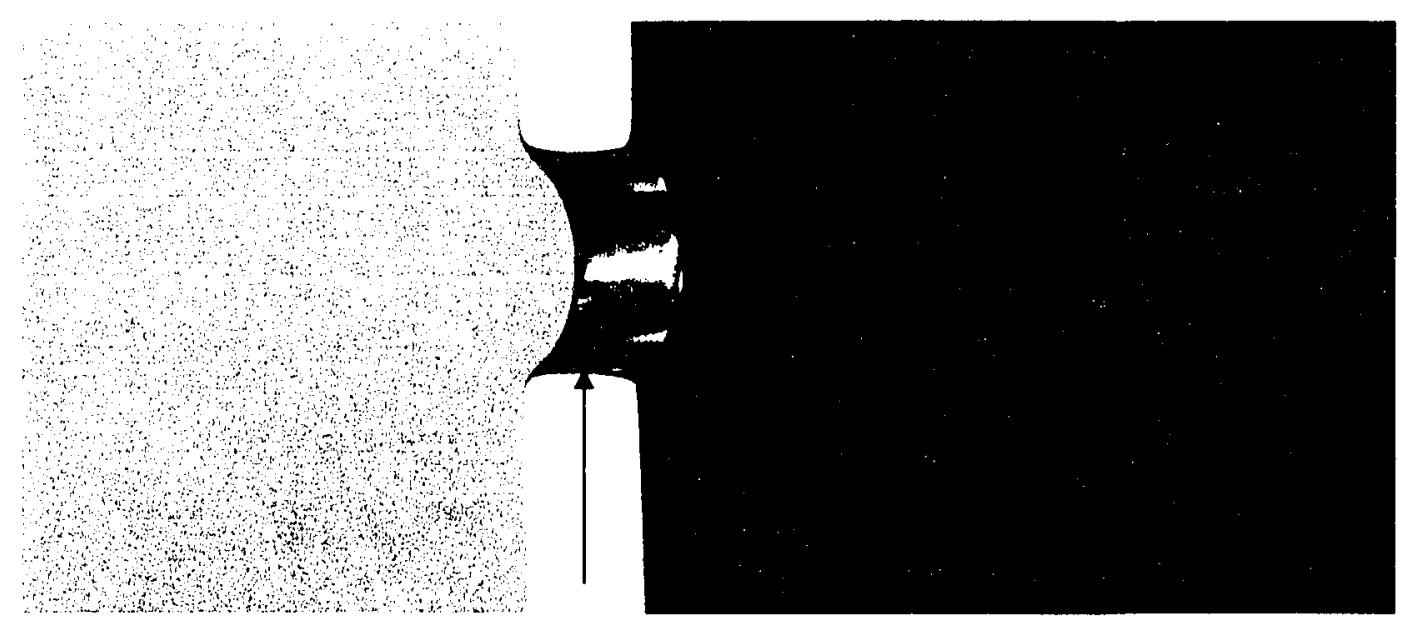

3. The handle area will be sandblasted to prevent slipping during the fatigue testing. First, the sample must be taped as shown below to provide the area to be sandblasted. * Sandblasting will cause residual stress within the material. Therefore, only sandblast the area that will be gripped and do not sandblast for too long to minimize the residual stresses.

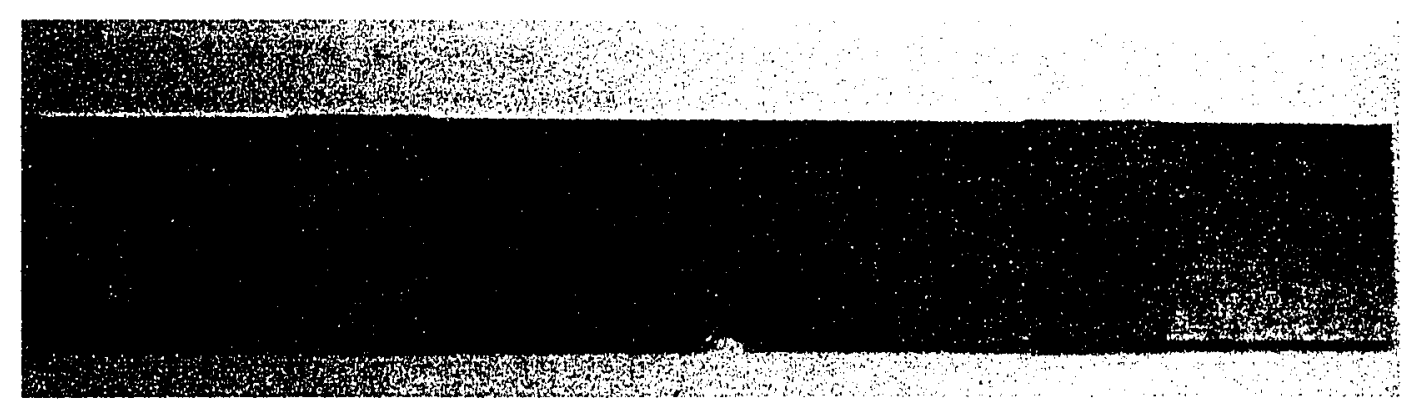


4. Load the sample through the door shown below.

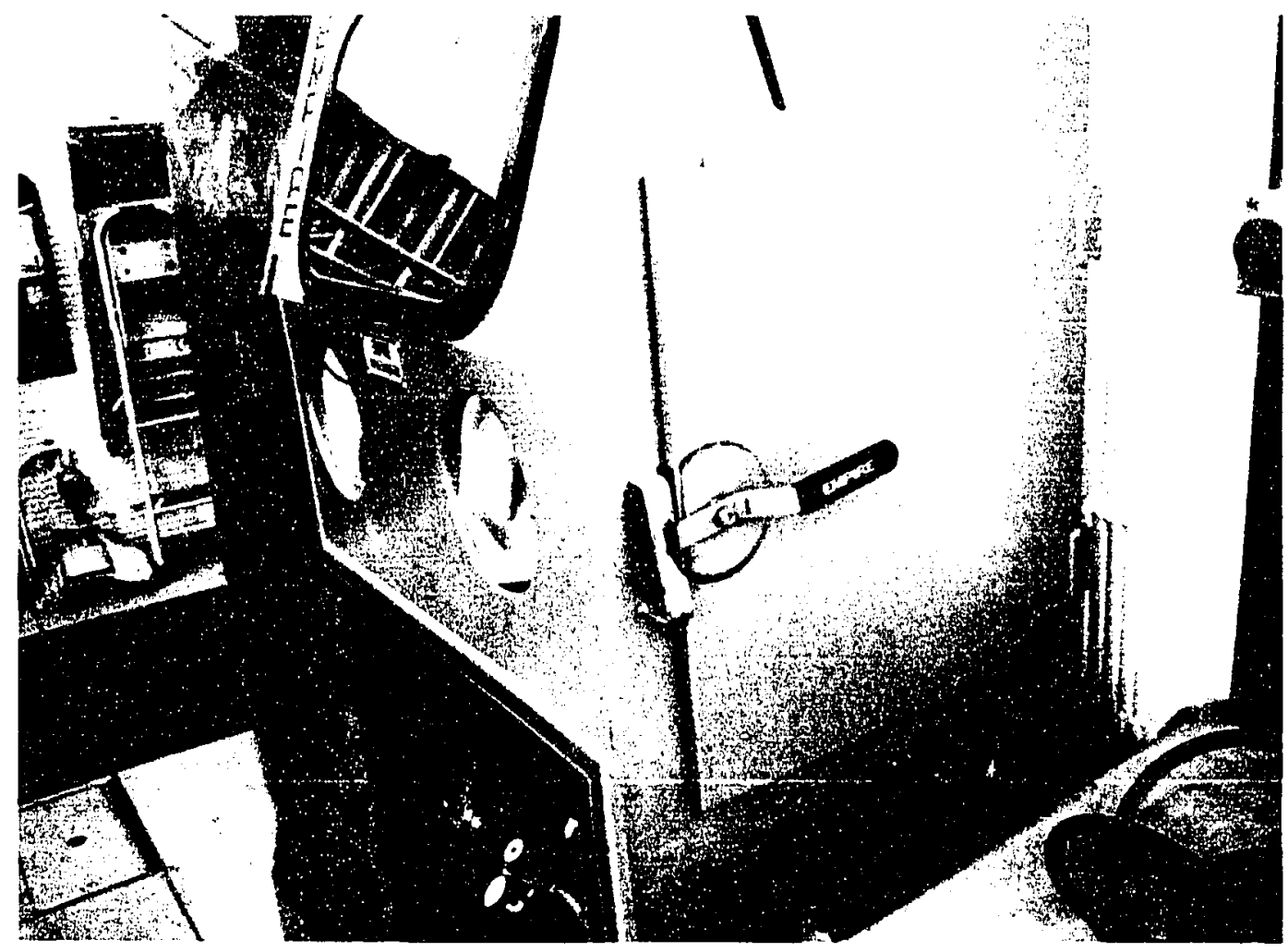

Turn the handle counter-clockwise and pull to open the door. Load the sample in and reverse the instructions to close the door. * Please note that a dust mask and a lab coat should be worn at all times during the sandblasting process.

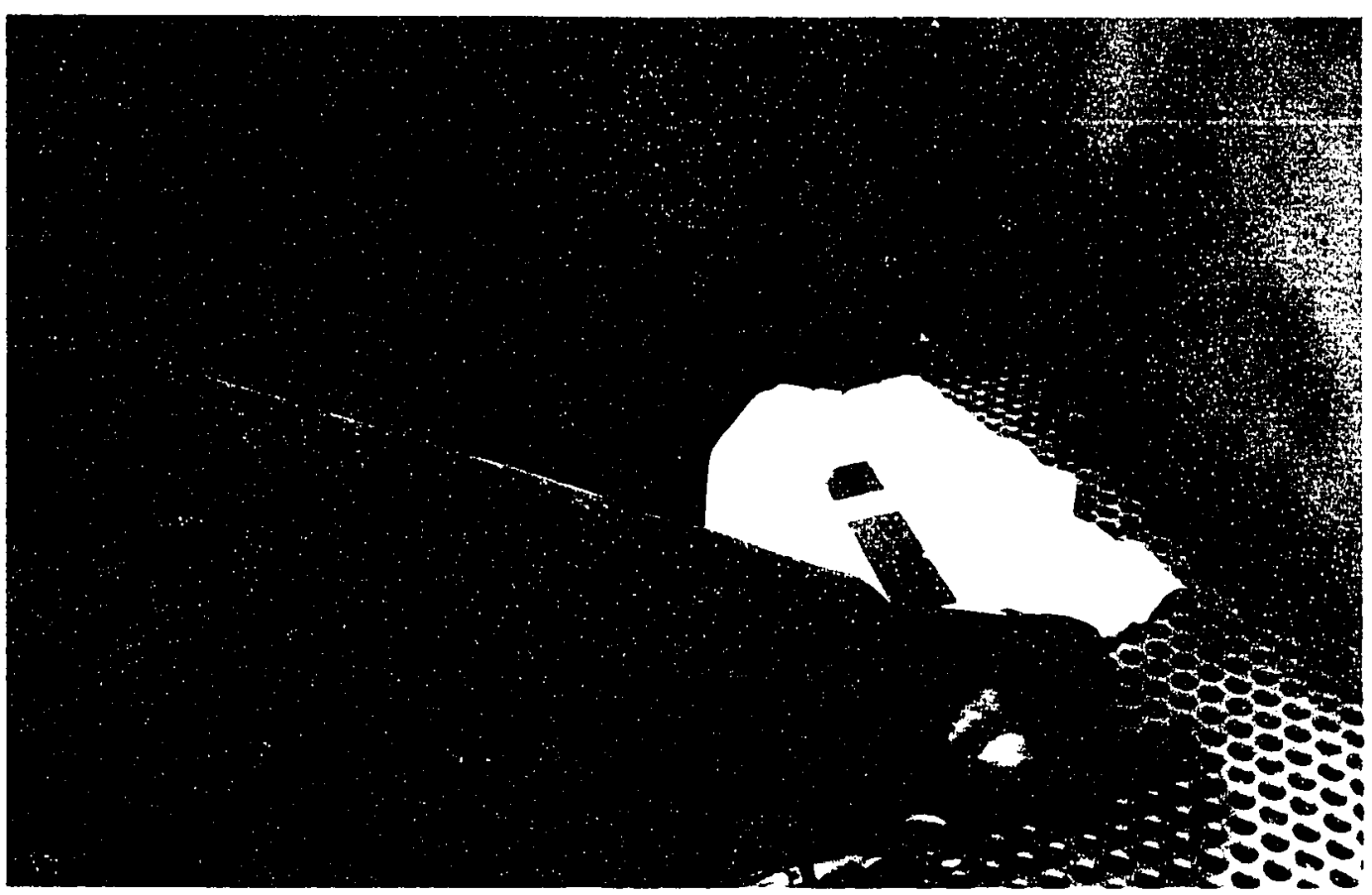


5. The sandblaster will need to be connected to the compressed air supply.

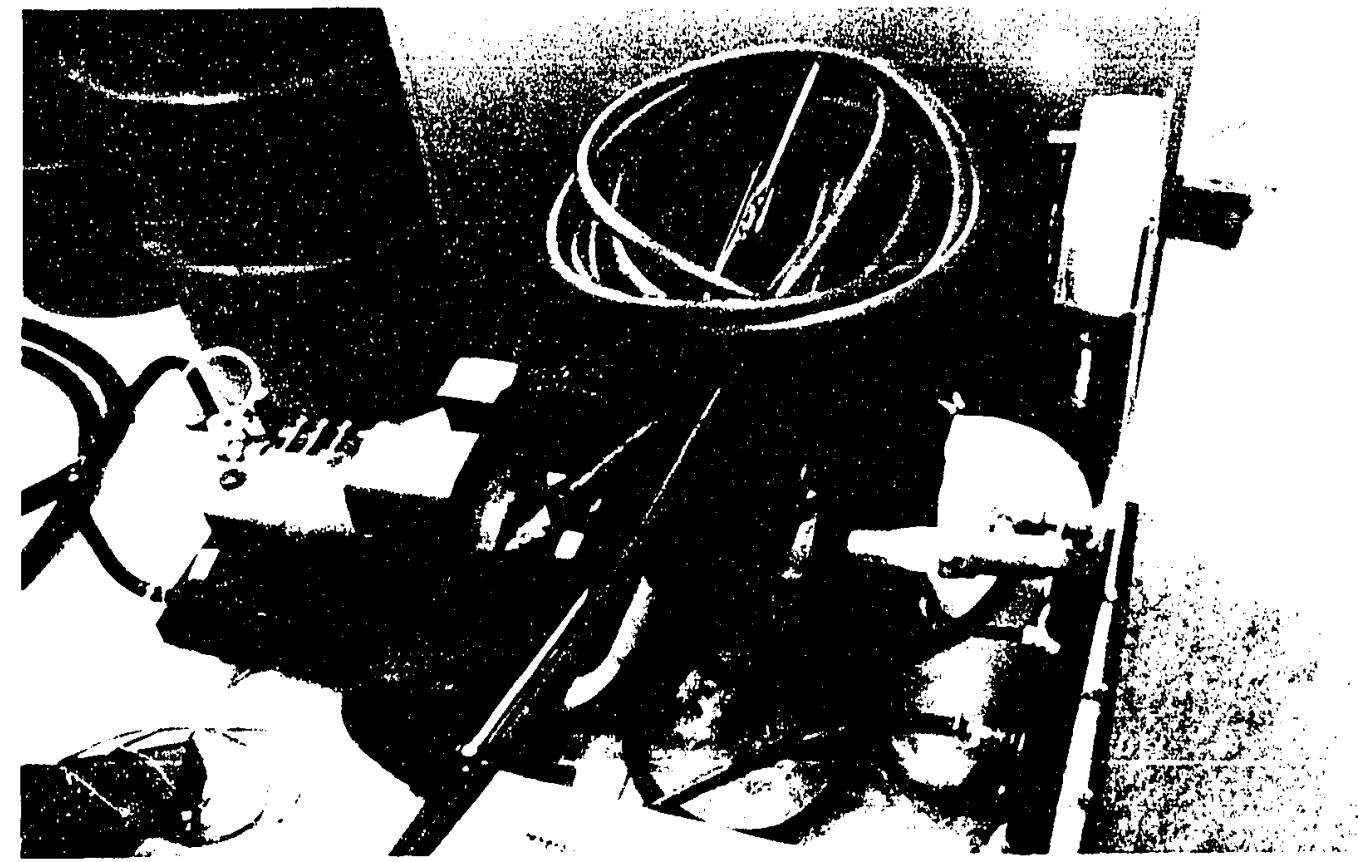

Connect the air supply to the sandblaster as shown below:

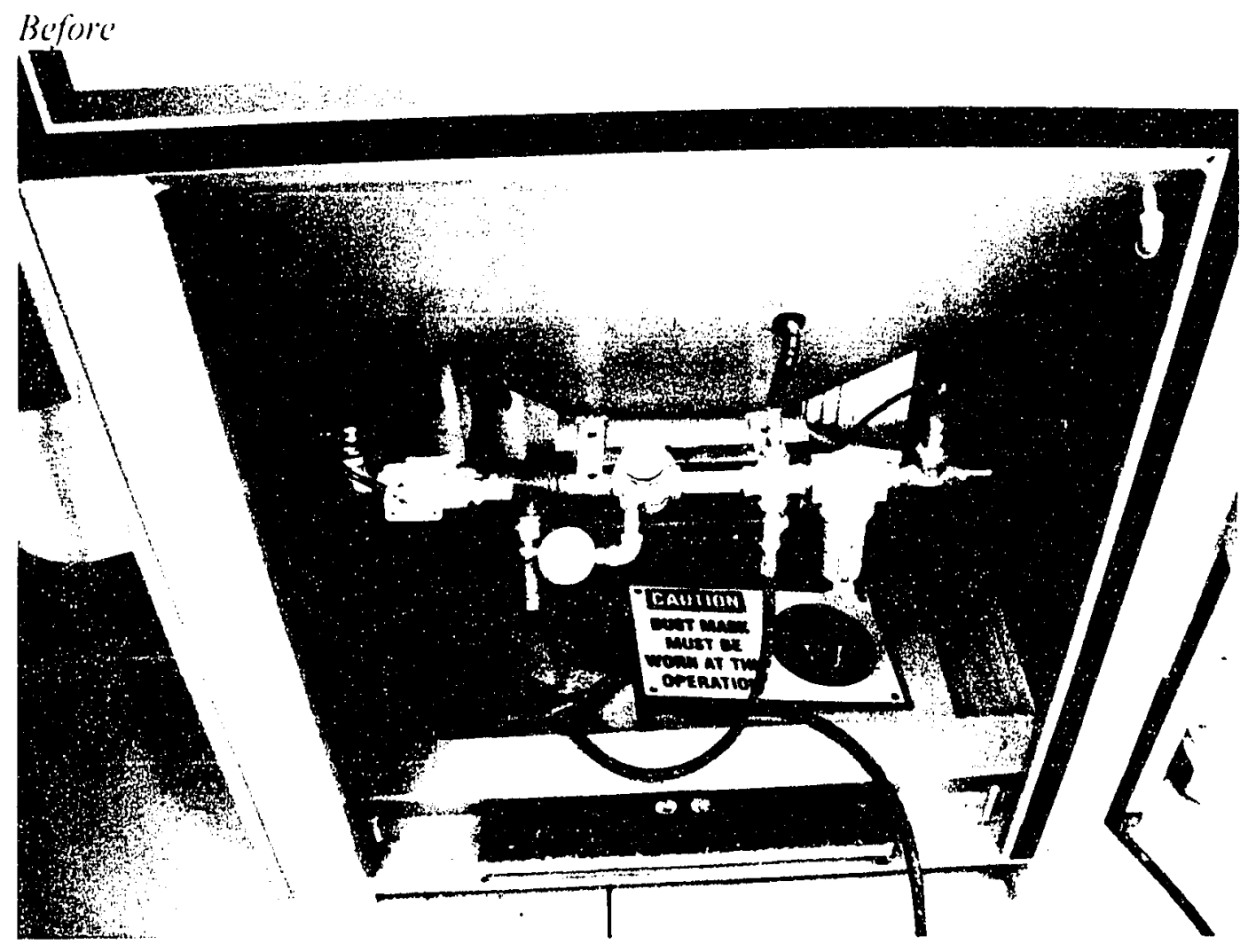




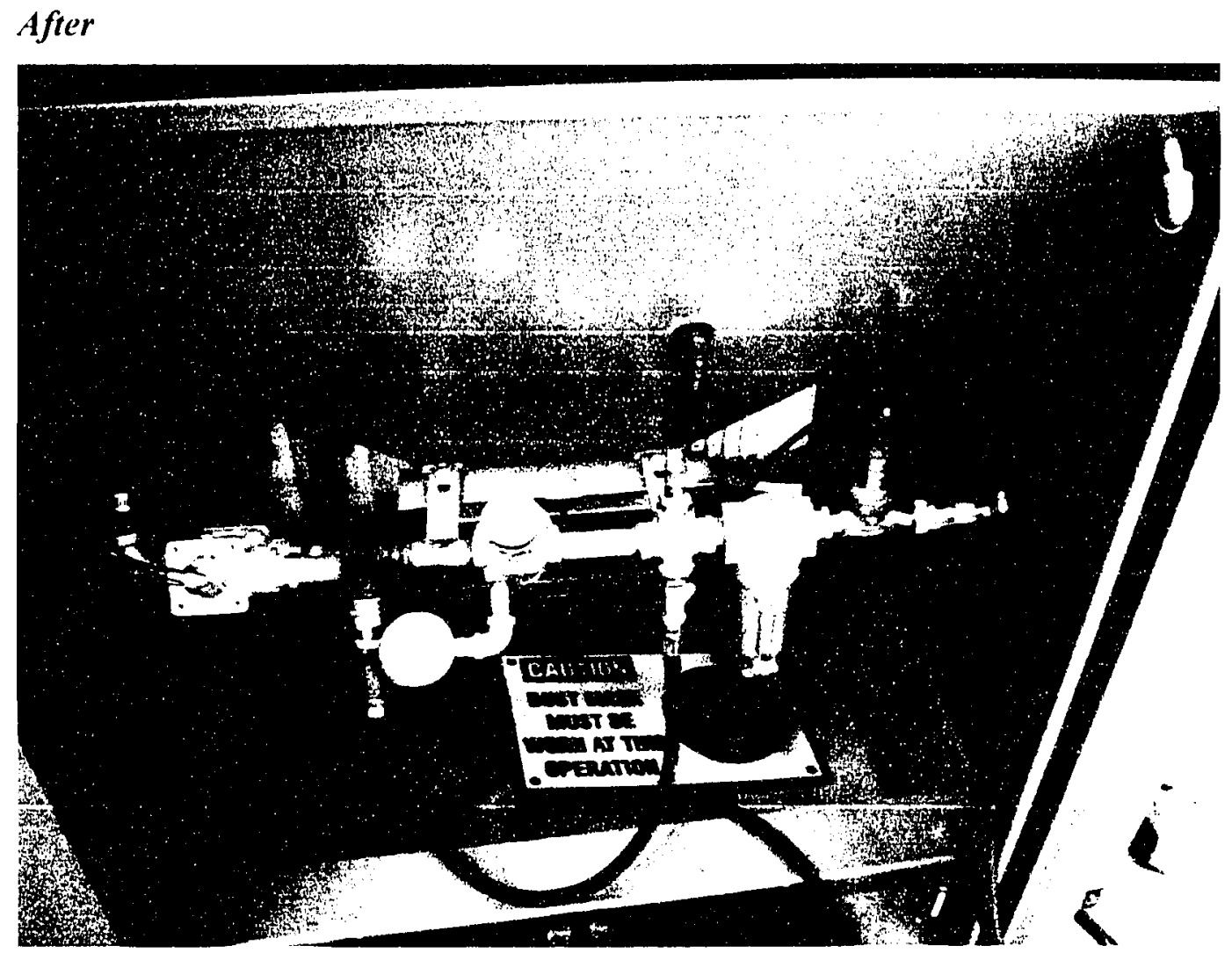

The pressure must be turned on. Before turning it on, the pressure gauge will read zero pressure.

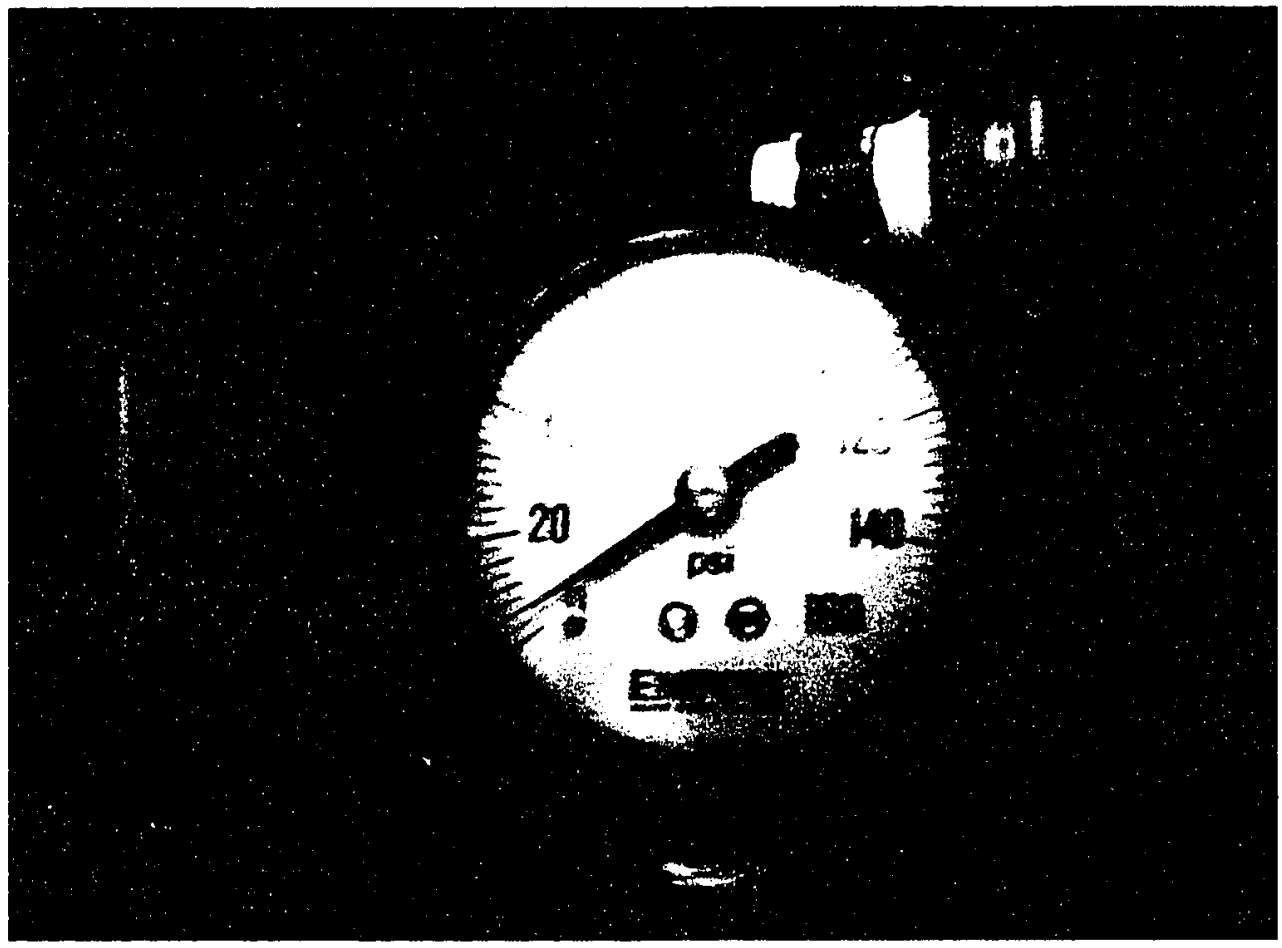


In order to turn on the pressure, the black know needs to be turned counterclockwise until it's fully open.

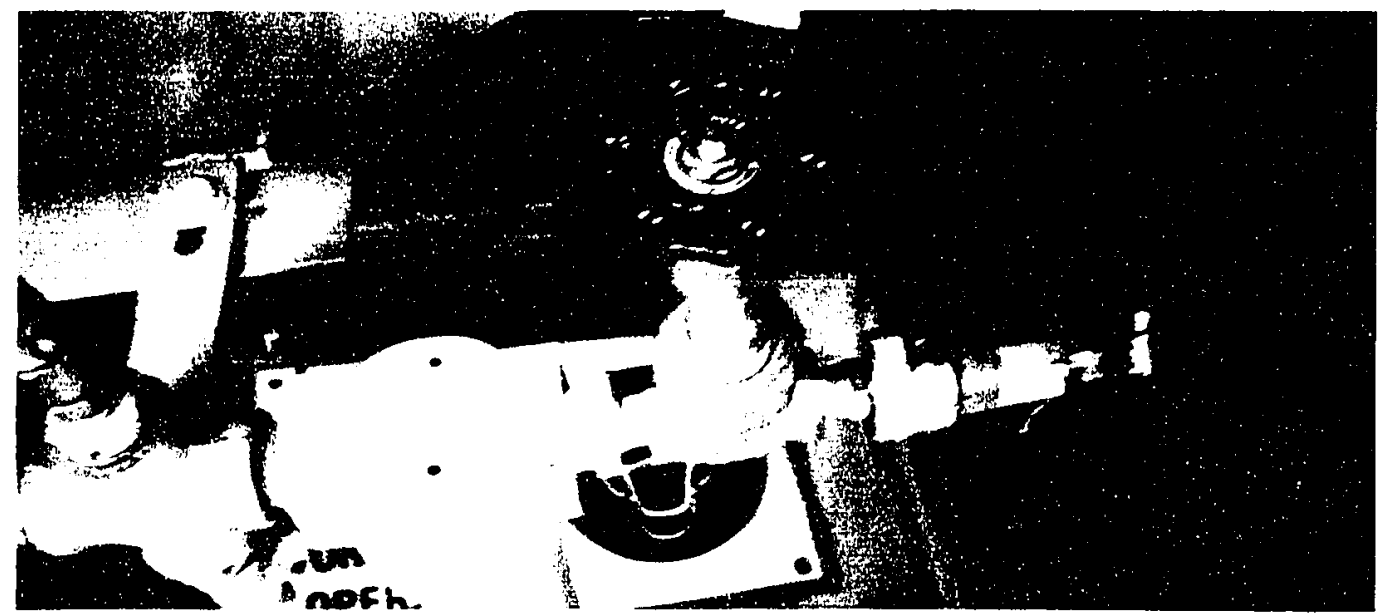

The pressure gauge should read somewhere between $100 \quad 120 \mathrm{psi}$.

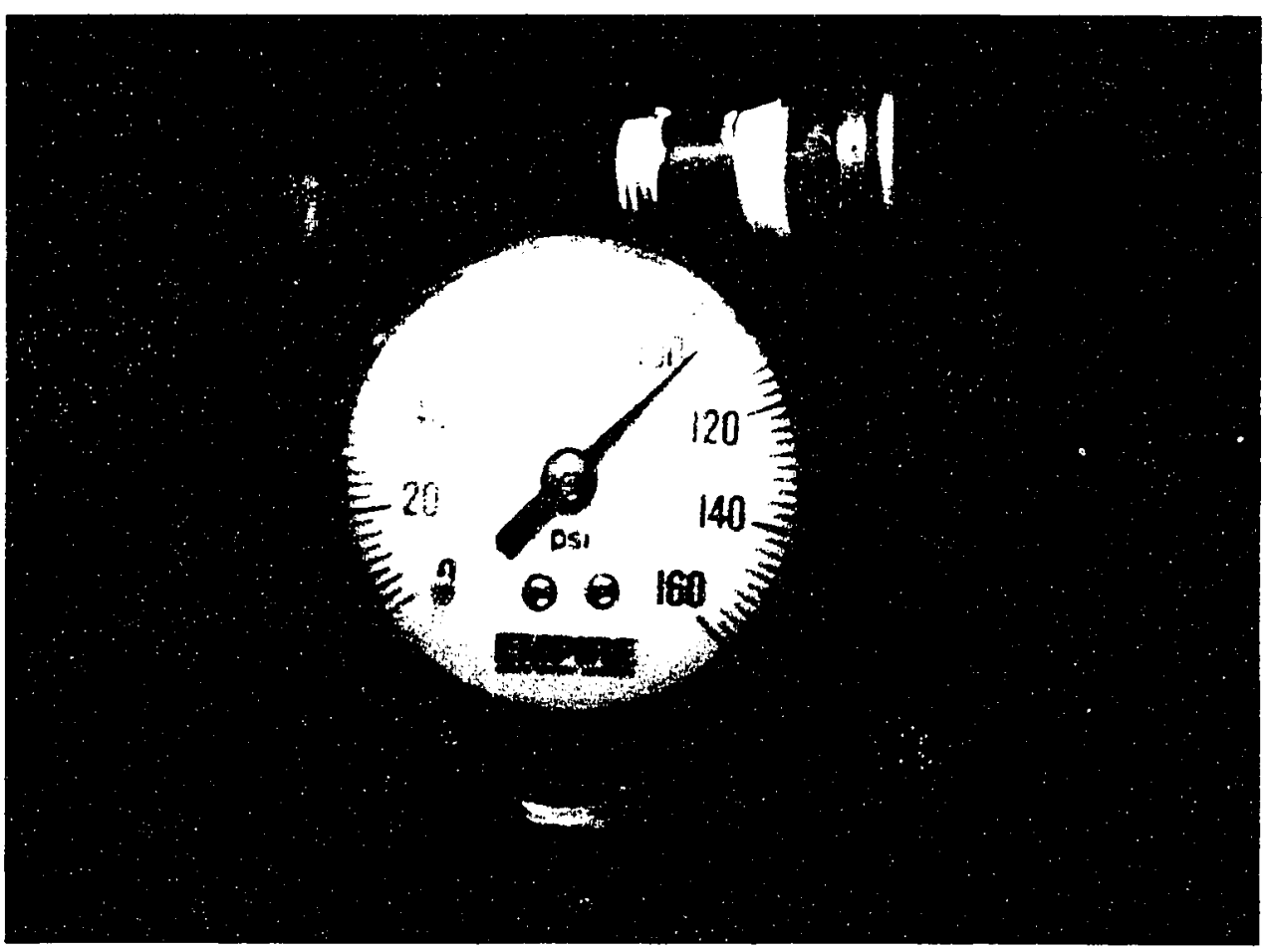


The sandblasting machine can be tumed on by the switch located on top of it.

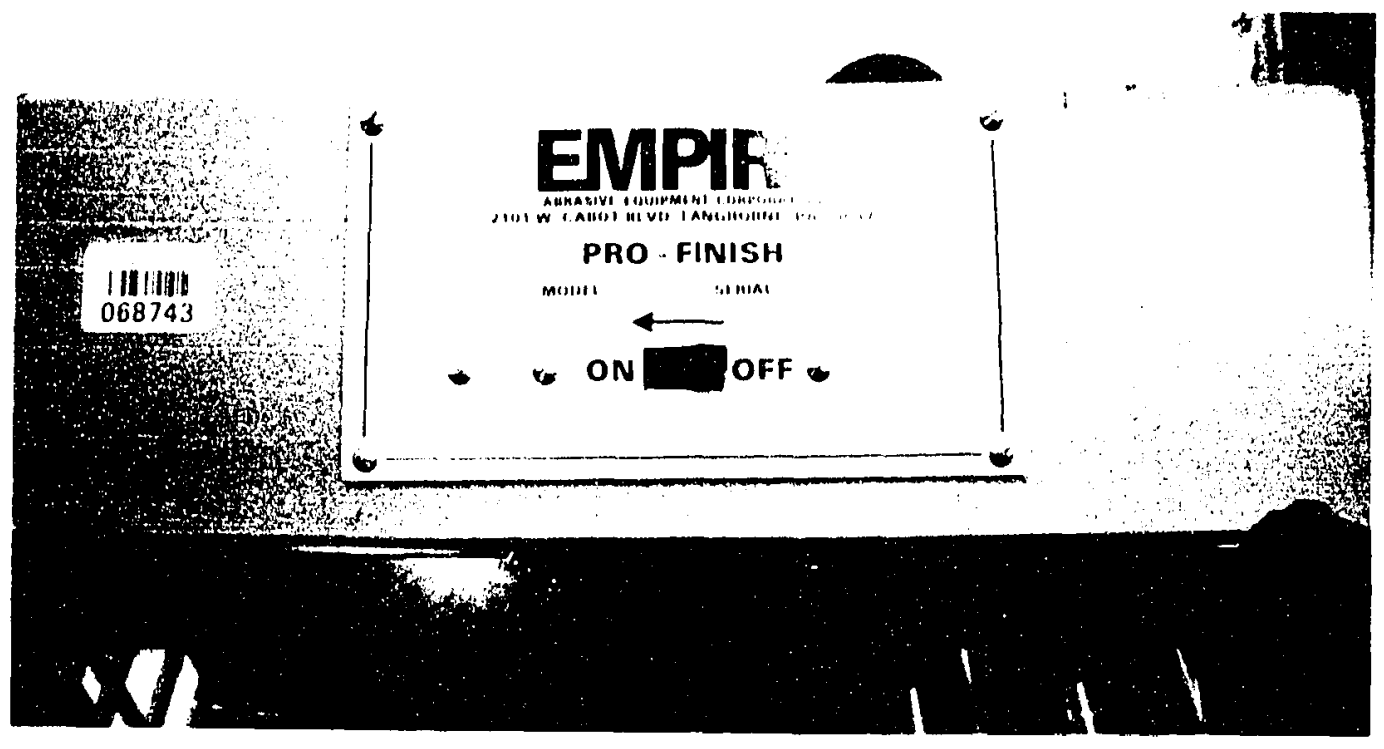

6. The sandblaster works by depressing on the foot pedal.

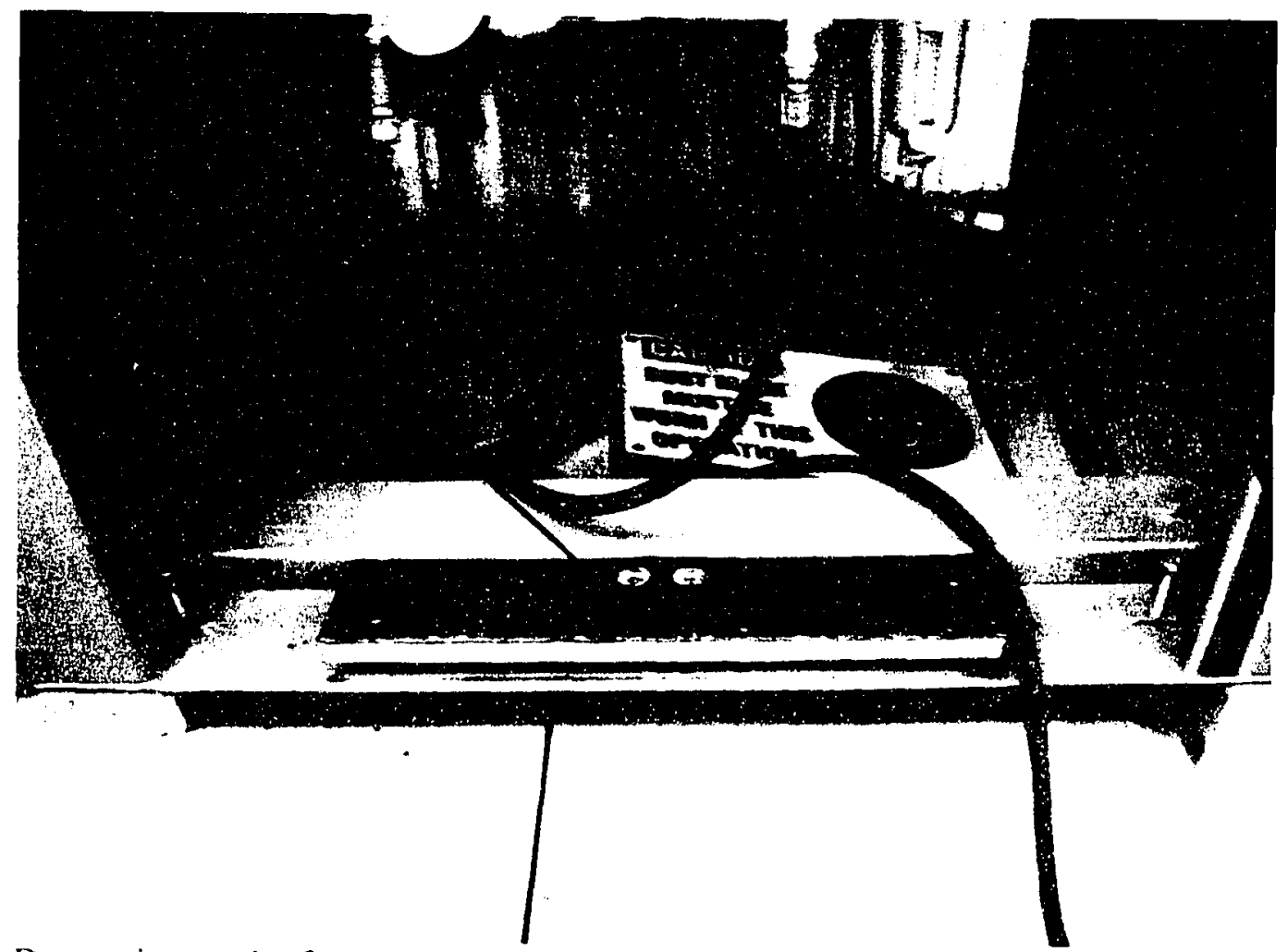

Depressing on the foot pedal will creale the blast of sand through the nozrle of the sand gun. 


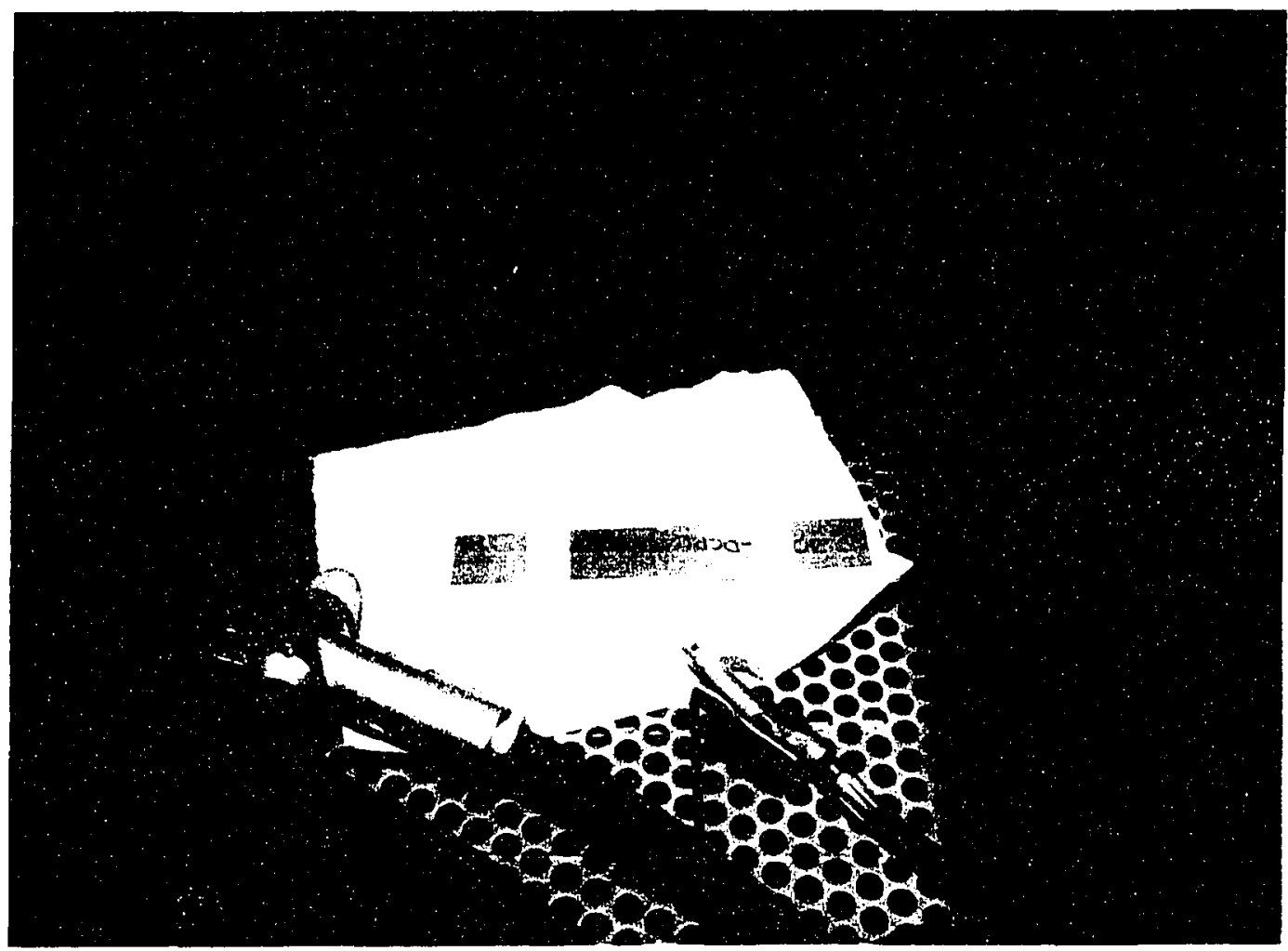

Lising SHORT BLiRSTS, spray over entire grip area until an even finish is achiered as shown.
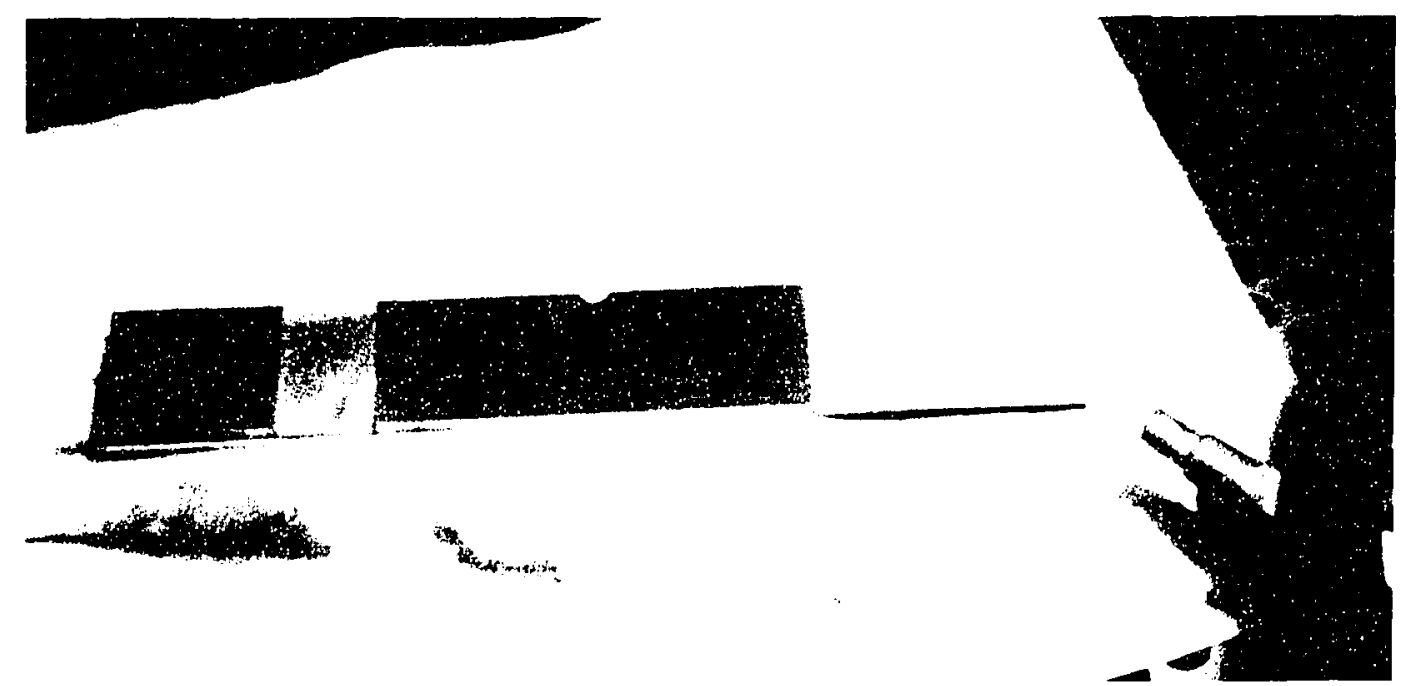

Repeat for the other side and then flip over to do the remaining two sides.

* Remember that sandblasting will create residual stresses and should be kept to a minimum.

7. Using the compressed air gun, spray over the entire chamber and sample to get rid of the sand within the chamber. 


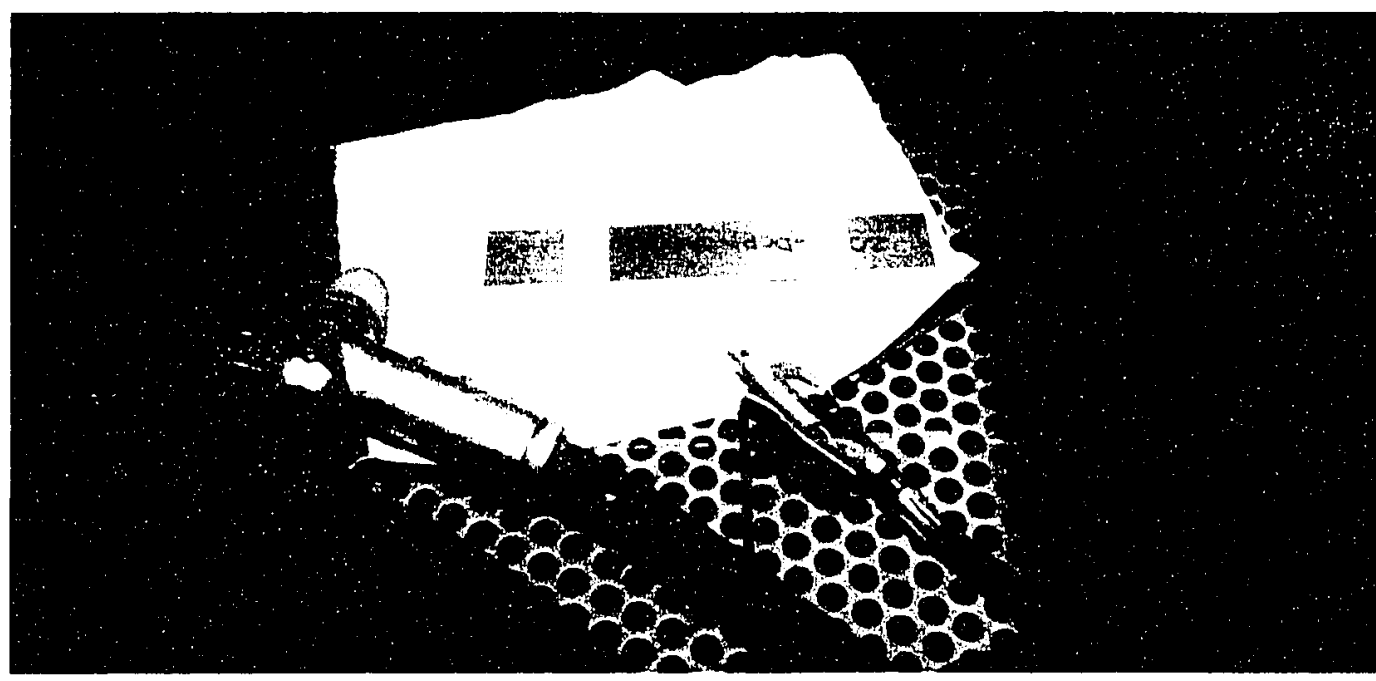

After the chamber is clean, turn it off by using the same switch on top of the sandblaster. Close the compressed air supply by turning the black valve clockwise. Spray the compressed air gun again to get rid of any remaining air pressure in the hose. It is now safe to remove the blue compressed air hose.

8. Open the door to the sand blaster as mentioned before and remove the sample. Remove the masking tape and use paper towel to wipe down the area near the notch to remove any sand particles that may interfere with viewing the notch under the traveling microscope. * Do this step gently to aroid scratching the notch. 
The sample should look like this.
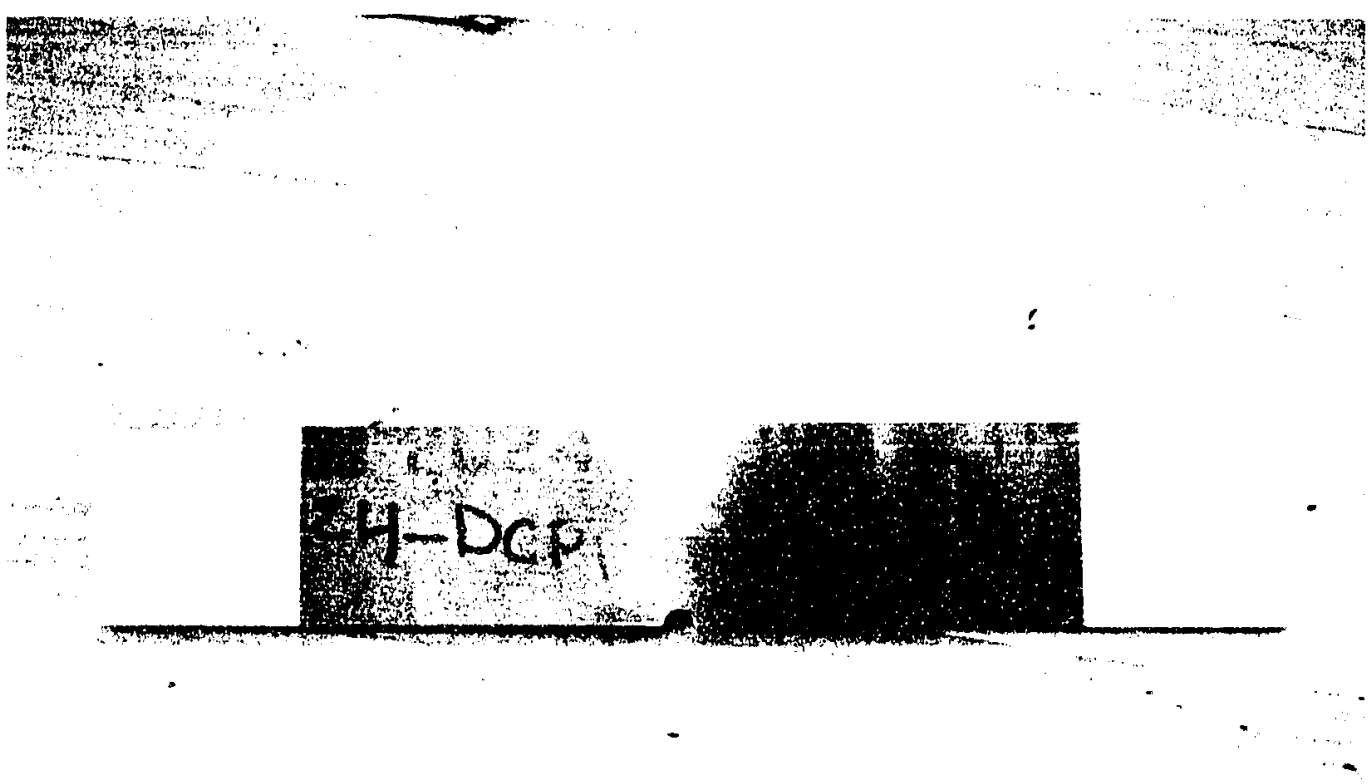

9. Place the sample in the lower grip. hush against the spacer and insert the aluminum shims with the SiC mesh paper as shown below. 


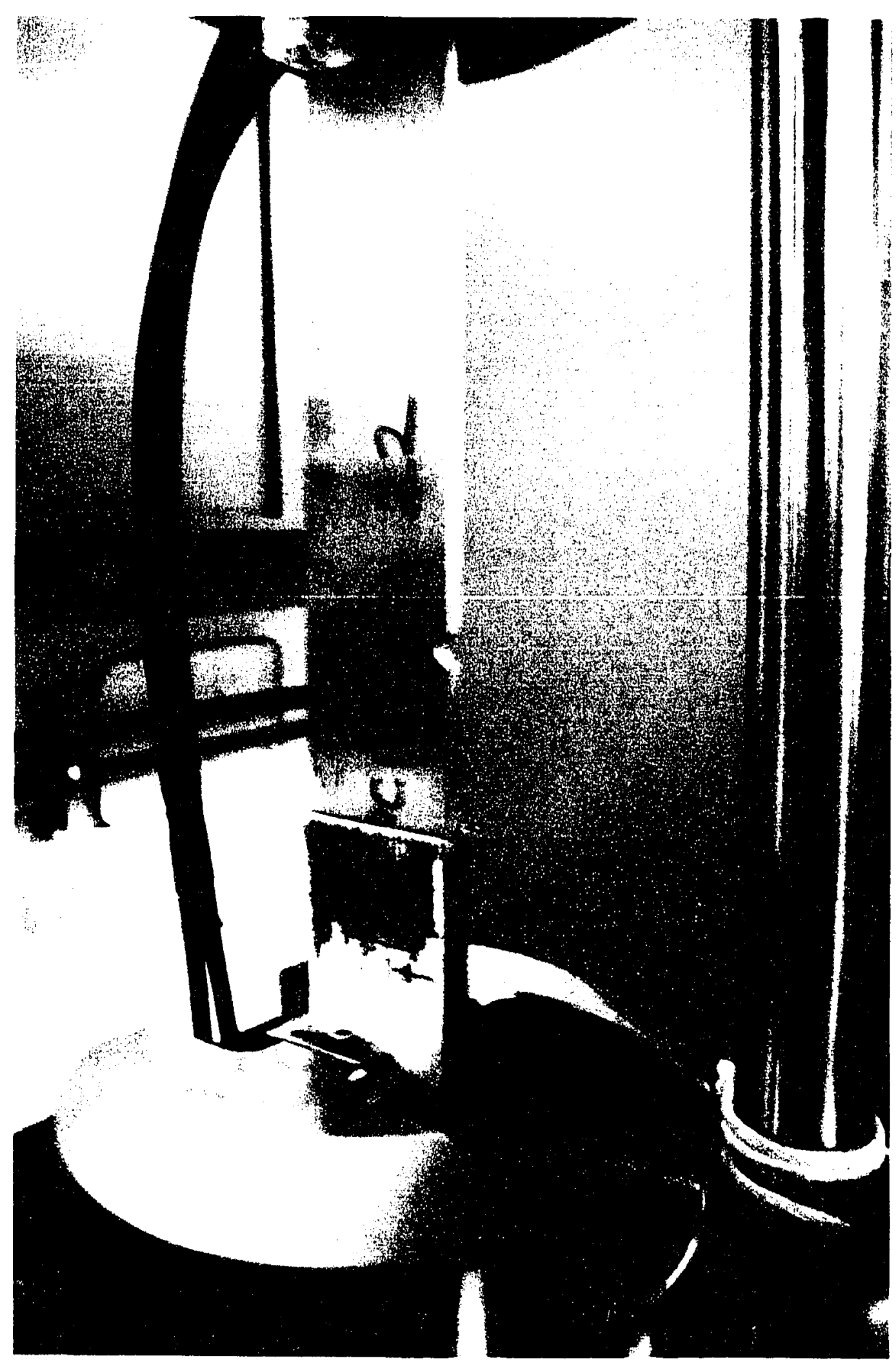


10. After the previous test was completed, the limits will have been surpassed and the program will still be "in progress." Therefore, the control pod will not be able to go into manual control until the following steps are followed.

Click on the new specimen button (\#1) and under the heading "Specimen", a new name will appear (i.e. 2024-depd-tk-01). Rename it to the specific specimen by typing it in the box (\#2).

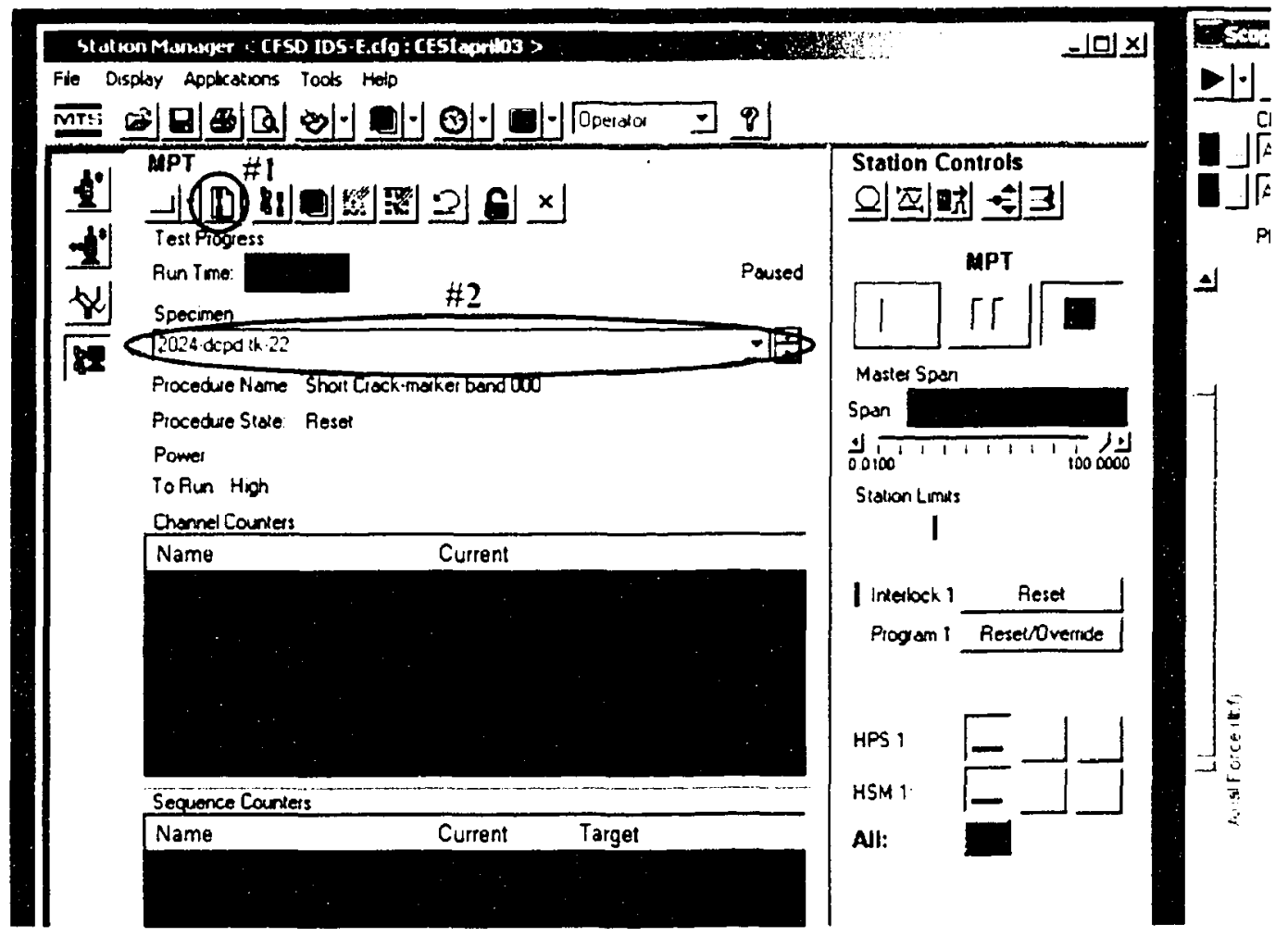

Before, proceeding back to the control pod, it is important to re-set the limits for loading the specimen so that there is a fail-safe from damaging the specimen or load frame during the sample loading operation. To bring up the limits, click on the "station setup" button (\#3) and change the upper force limit to a force a lot less than the maximum load during fatigue testing (i.c. $200 \mathrm{lbs}$ ). ( $\# 4$ ). 


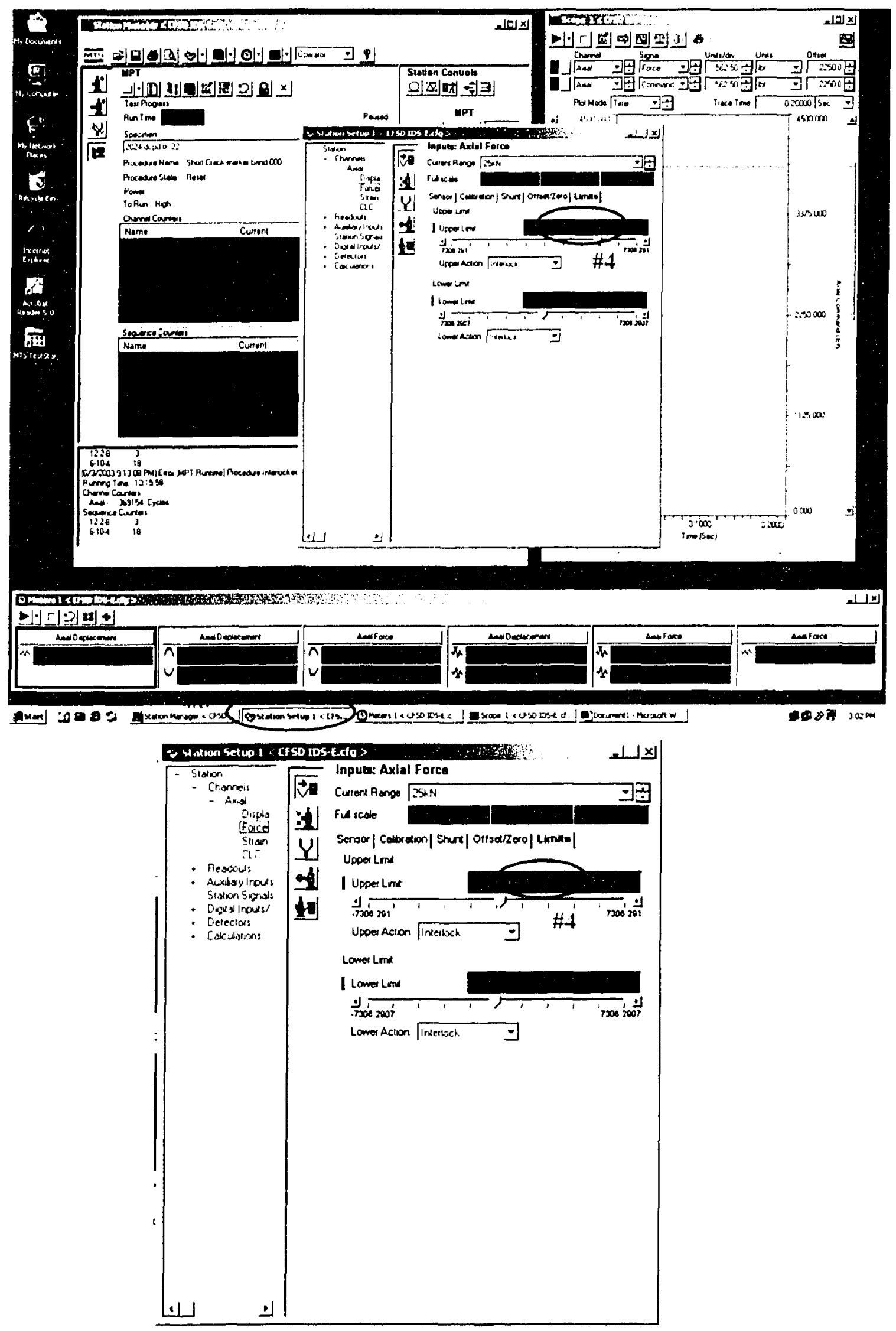


The displacement limits need to be disabled or else they will be tripped off during the loading operation. To disable them, click on the displacement button (\#5) to go to the displacement window and change the pull-down menus (\#6) from "Interlock" to "Disabled."

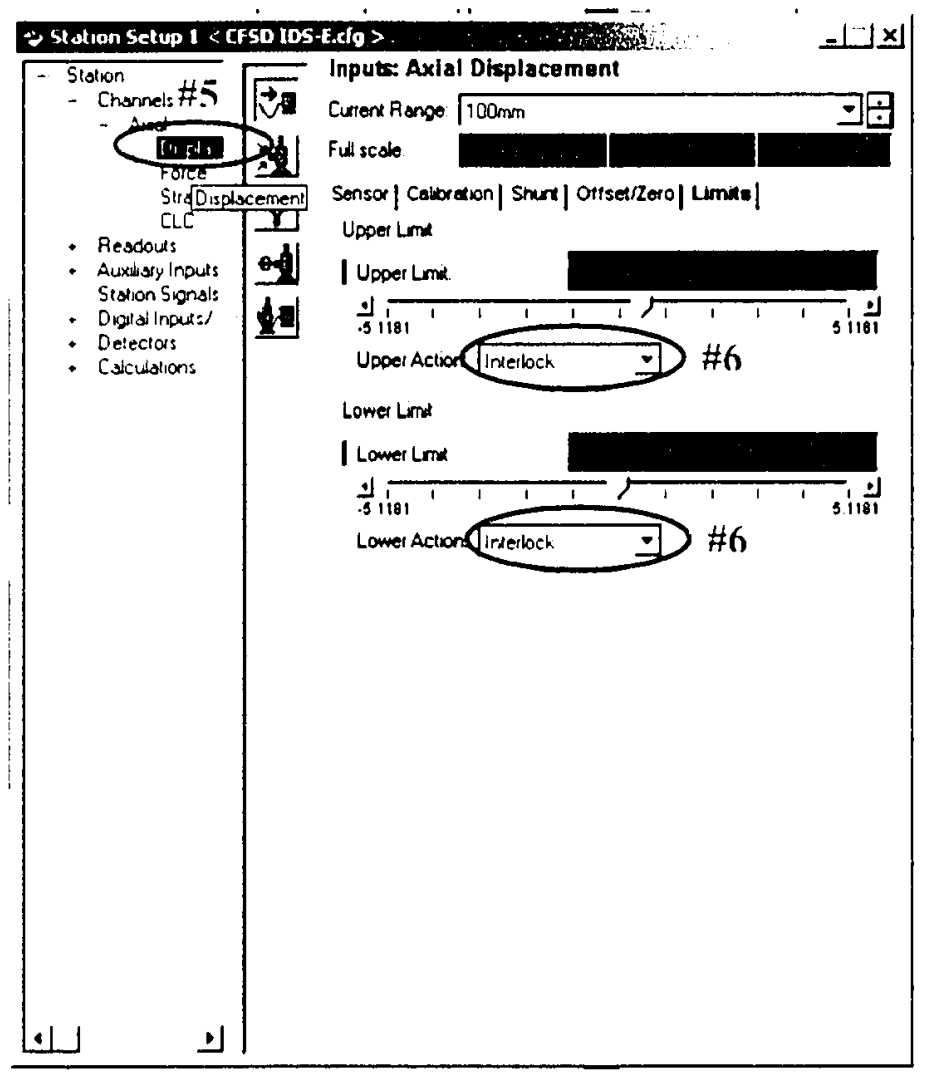




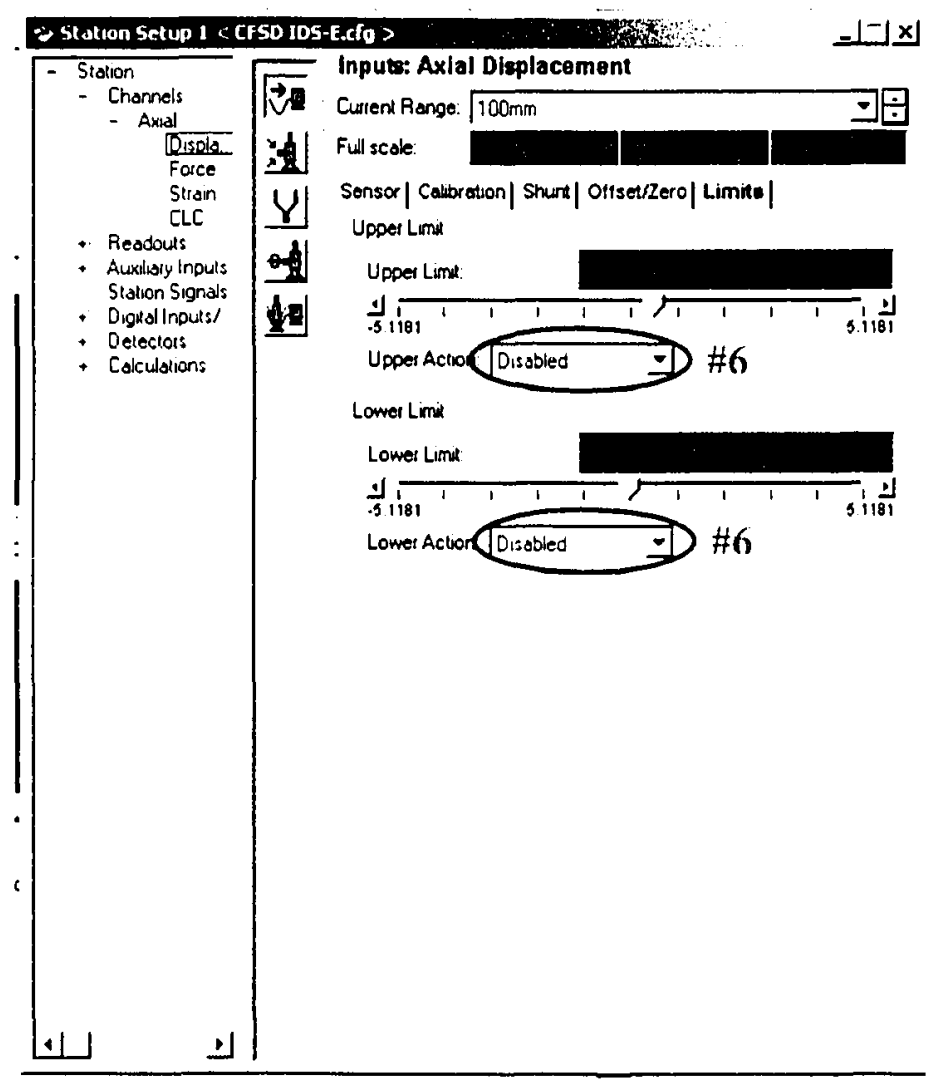

11. The control pod can now be set to manual mode. Firstly, press the reset button (\#7) on the control pod to remove the interlock. Once depressed, the red light should disappear. As can be seen by $\# 8$, the system will still be in force control and it should be changed to displacement control. Press the F4 button (\#9) until the "Manual Ctrl" reads "Displacement" and then press the "Enable/Disable" button (\#10) twice to put the system in displacement control. The "Axial" should now read "Displacement."

Turn on the hydraulics by pressing the "Low" and then the "High" for the "HPU" (\#11) and repeat for the "HSM" (\#12). Press the "Enable/Disable" button (\#10) once to enable the manual control. With the manual control enabled, the sample will move up and down by turning the knob clockwise (tension) or counterclockwise (compression). 


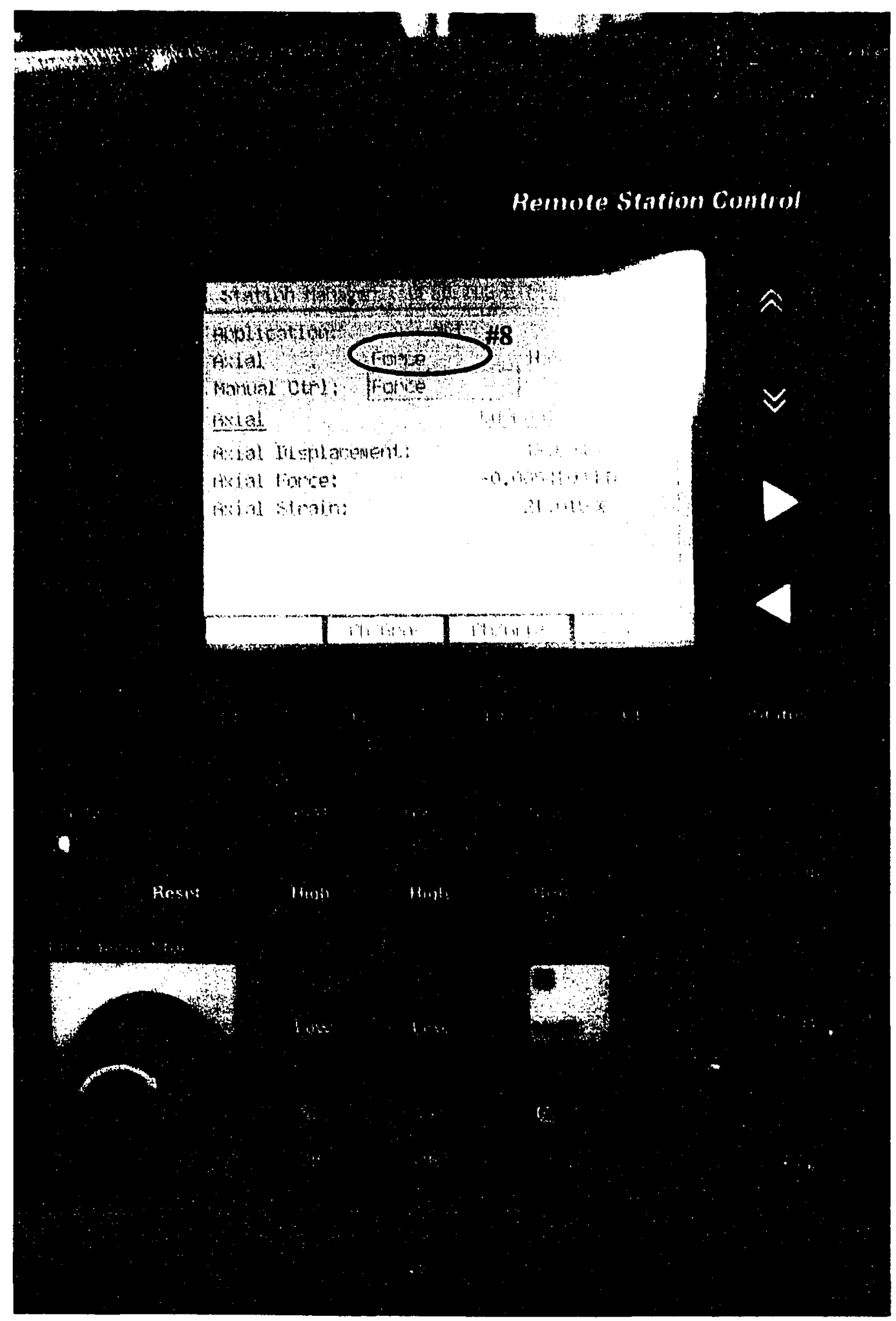

Raise the sample so that the top of the specimen is nearly touching the upper grip.

* Be careful not put any compression load on the sample! 


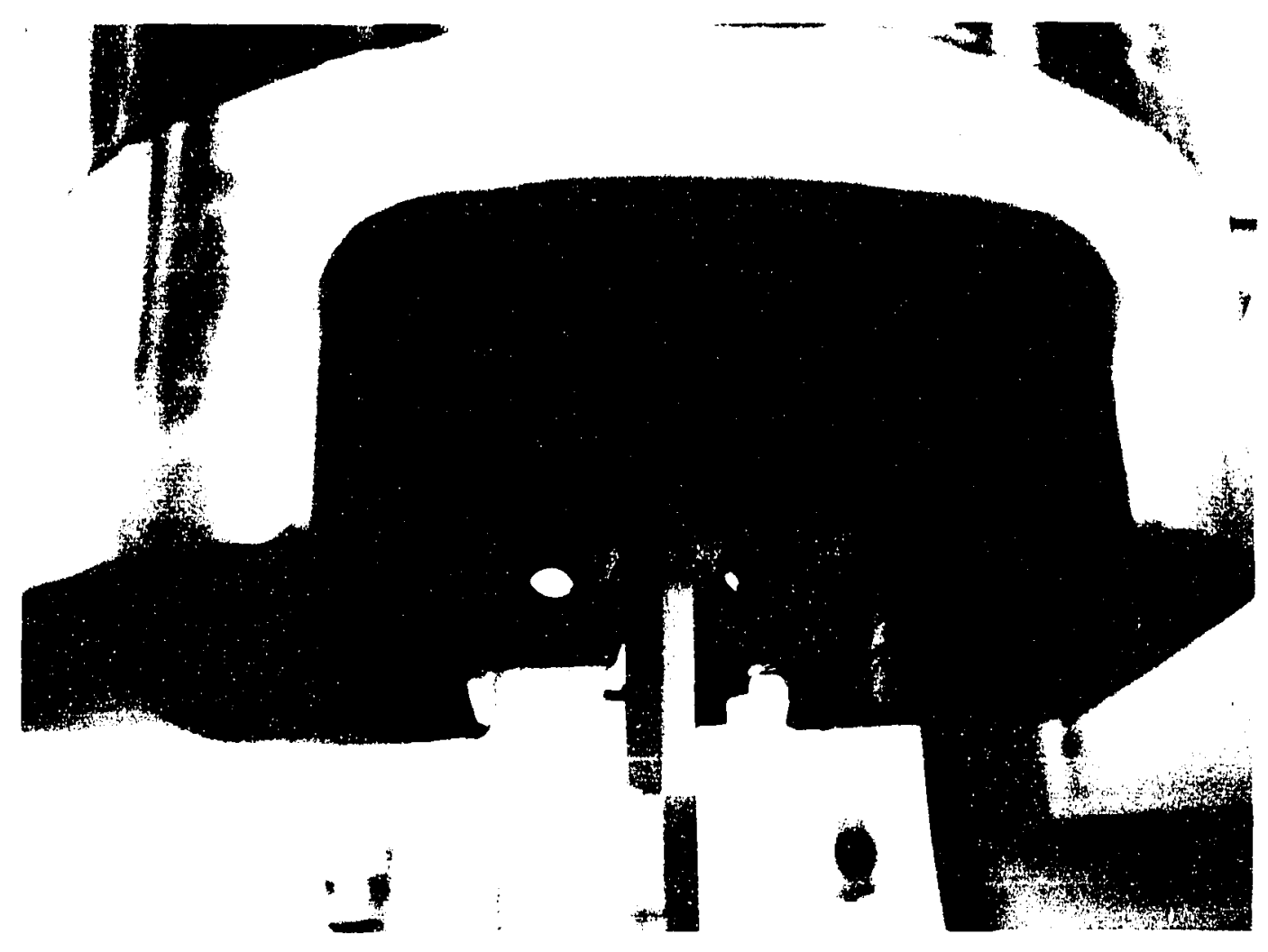

Press the "Enable/Disable" button ( $\# 10)$ again to turn off manual control and insert the aluminum shims with the SiC mesh on both sides of the specimen in the top grip. After the shims are in, and BEFORE the specimen is gripped, check that the top and bottom of the sample are flush against the two spacers as shown below. 


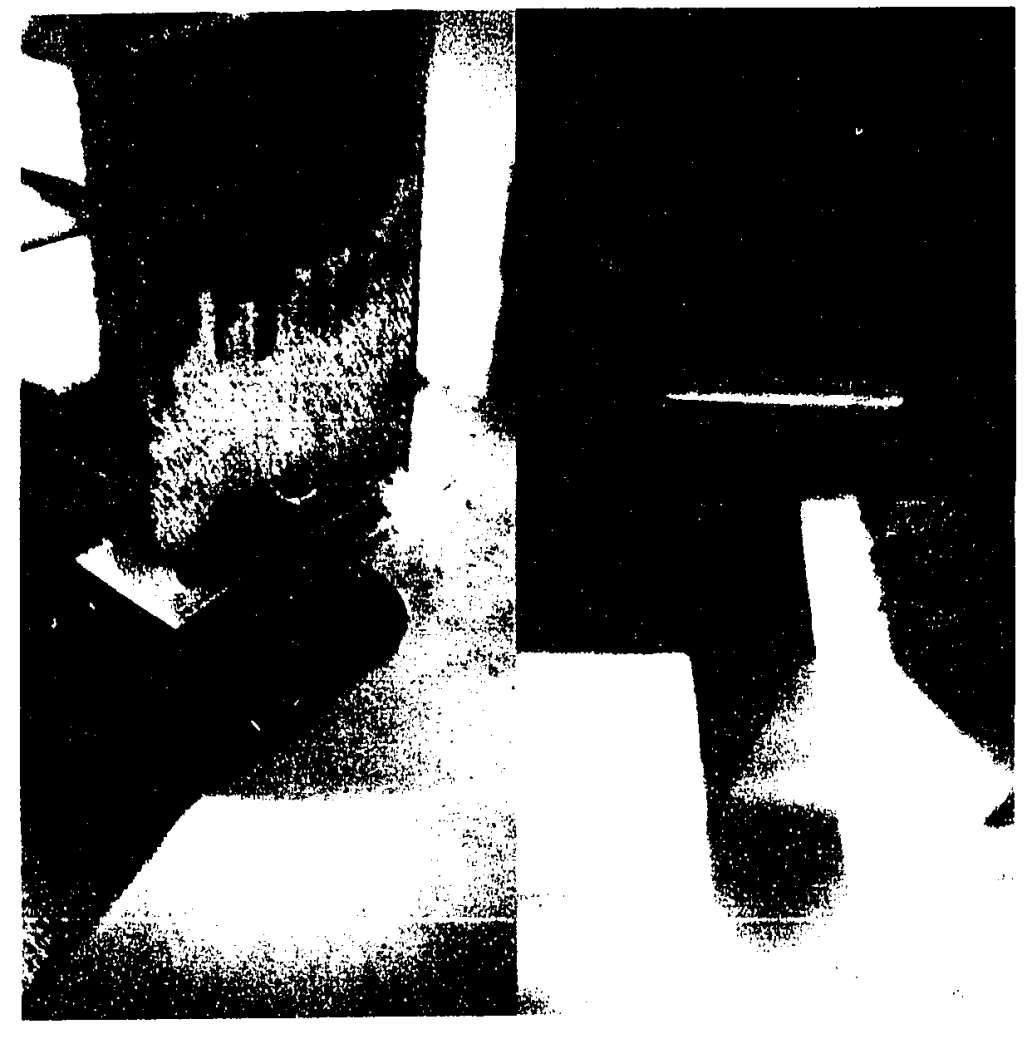

After checking the spacers, grip the bottom grip by turning the bottom lever clockwise. 
Before

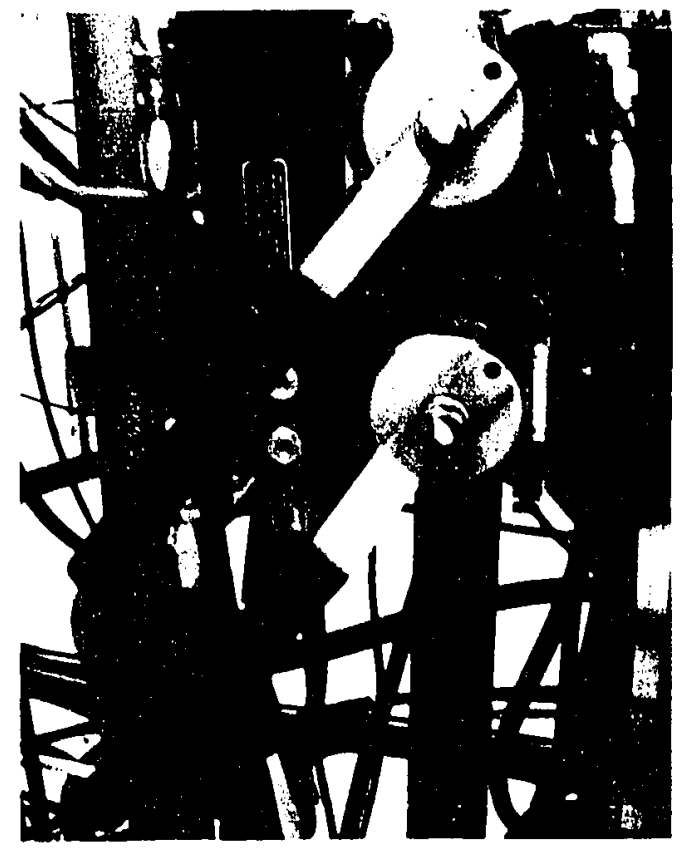

After

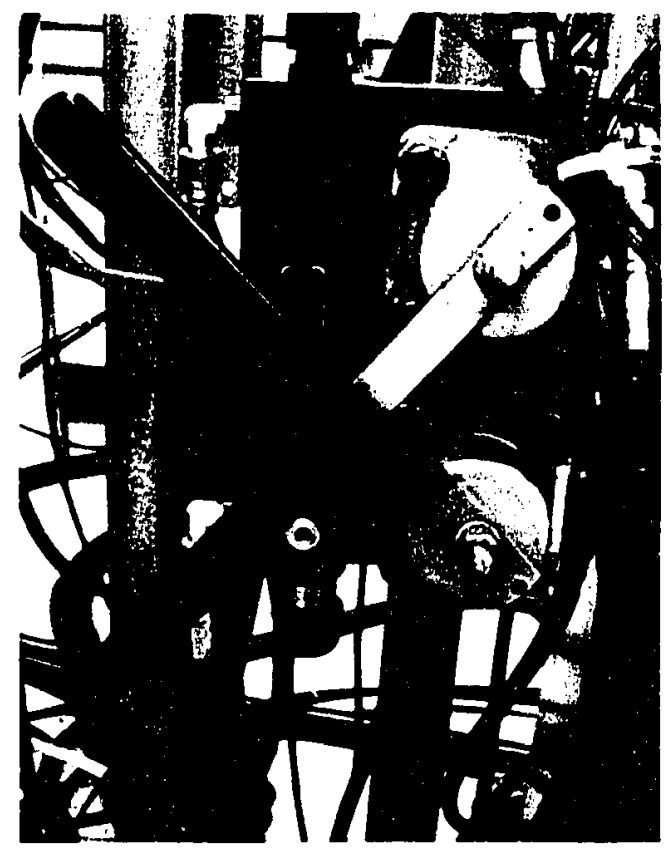

BEFORE gripping the top grip, the control mode must be switched to force control to ensure no compressive or tensile forces are applied to the sample. Press the F4 button (\#9) to switch the "Manual Ctrl" to read "Force". Pressing the "Enable/Disable" button (\#10) to switch the "Axial" to read "Force" will put the system in force control. With the system in force control, turn the other lever to grip the top grip in the same manner as the bottom grip. The levers should now look like this: 


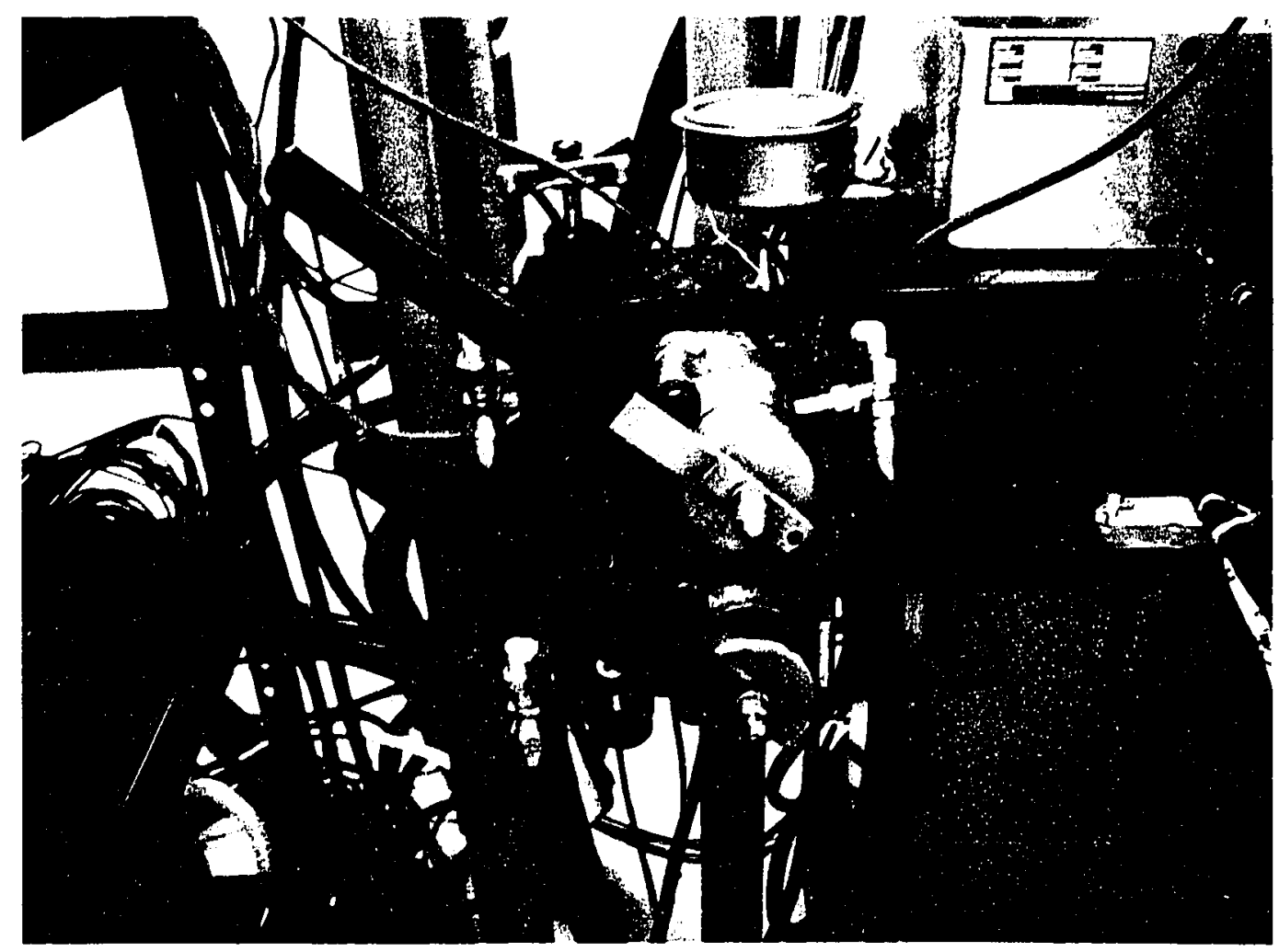

Adjust the force so that the sample is slightly in tension. Once the force is adjusted, press the "Enable/Disable" button (\#10) to disable the pod control.

12. Auto-offset the displacement by pressing the arrow buttons (\#13) to scroll down to "Axial Displacement" and press the FI button ( $\# 14)$.

13. Before starting the test, the limits must be adjusted for the testing conditions. Using the same methodology for resetting the limits before, set the upper force limit so that it is above the maximum load and re-enable the displacement limits to "Interlock." 

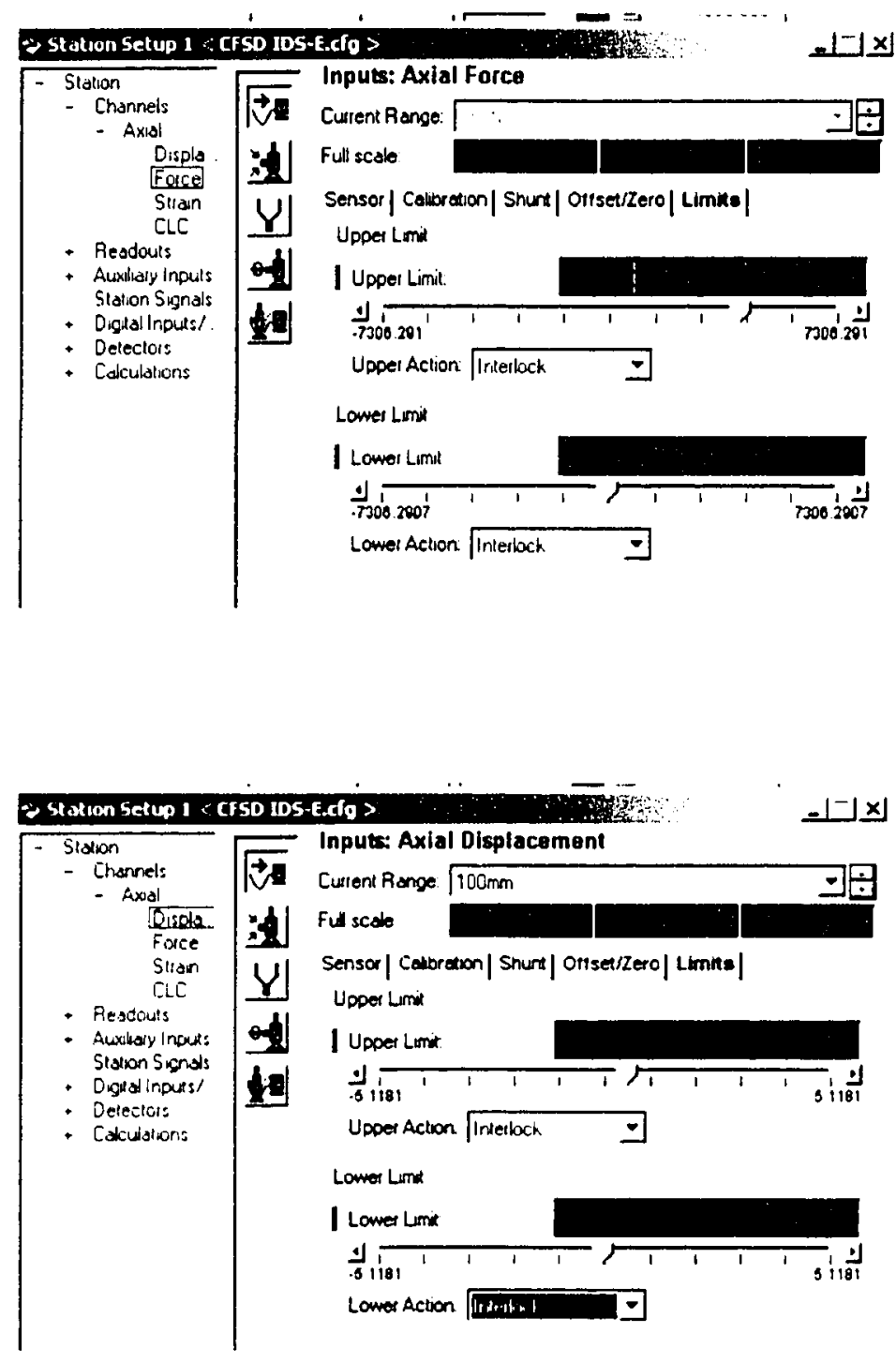

14. Before beginning the fatigue test, line up the traveling microscope so that the notch of the specimen is in view and adjust the focus so that the deepest part of the notch is clearly visible as shown below. 


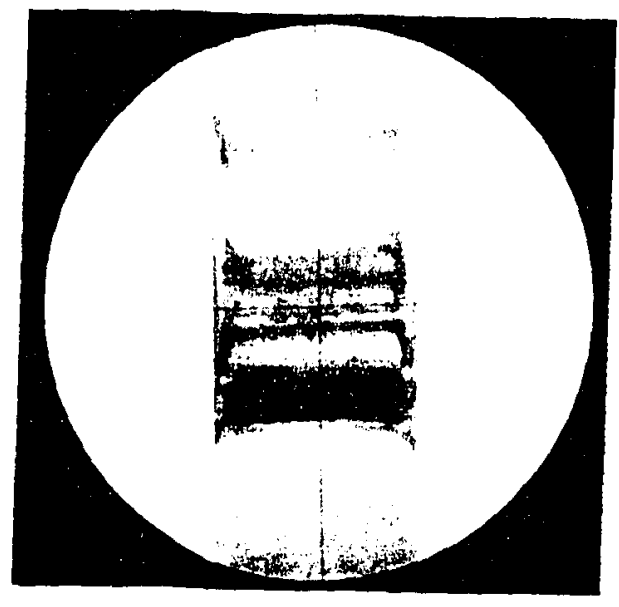

15. Write down all critical information about the test in the logbook.

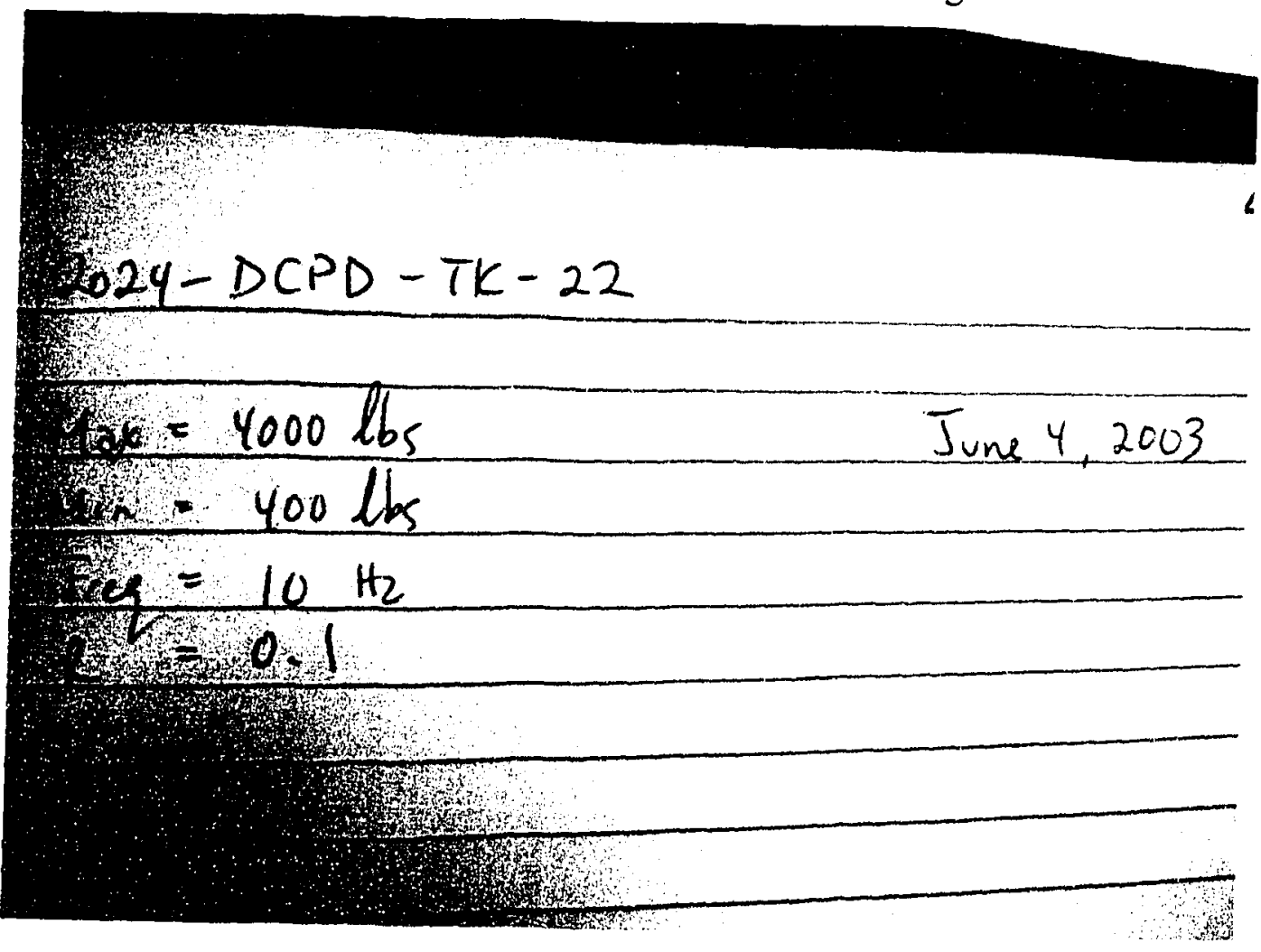

16. Start the test by pressing on the " "button (\#15) 


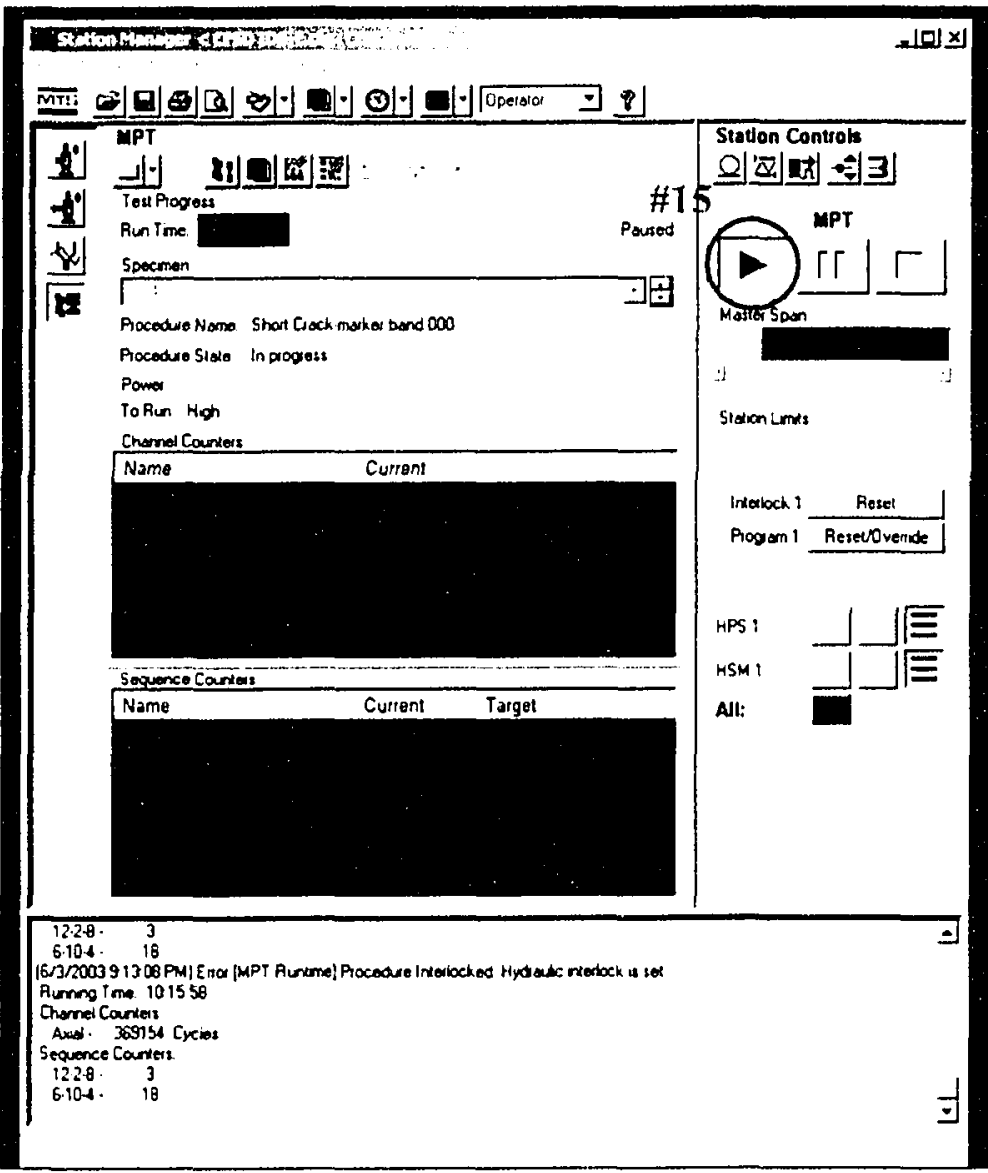

17. Once the cyclic loading is in progress, press the reset button (\#16) on the bottom meter window. 


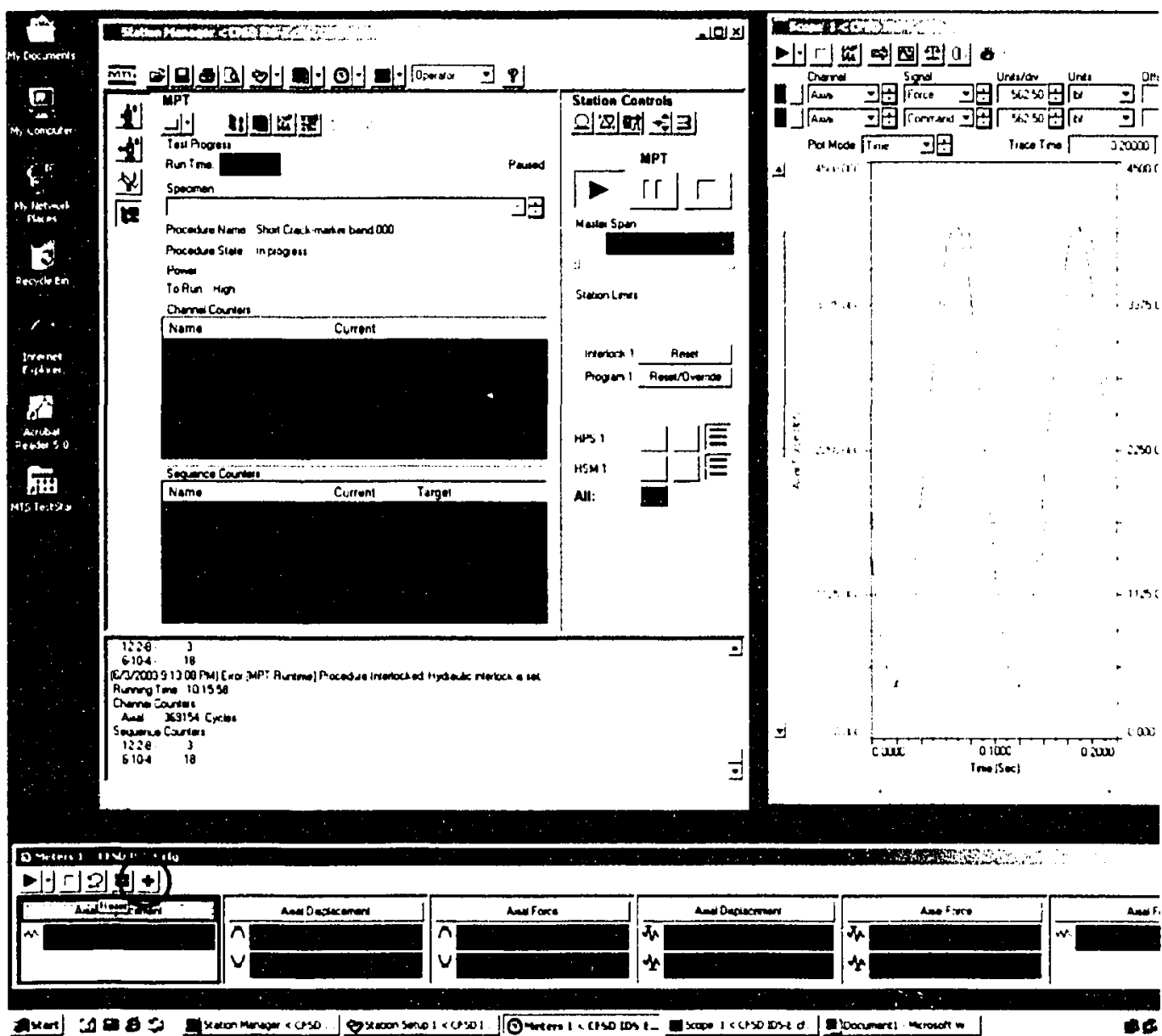

18. After the test has been completed, record the cycles to failure in the logbook. 


\section{APPENDIX D - EPD Testing Procedure}




\section{Procedure for Electrical Potential Drop (EPD) Fatigue Testing for Nucleation and}

\section{Short Crack Study}

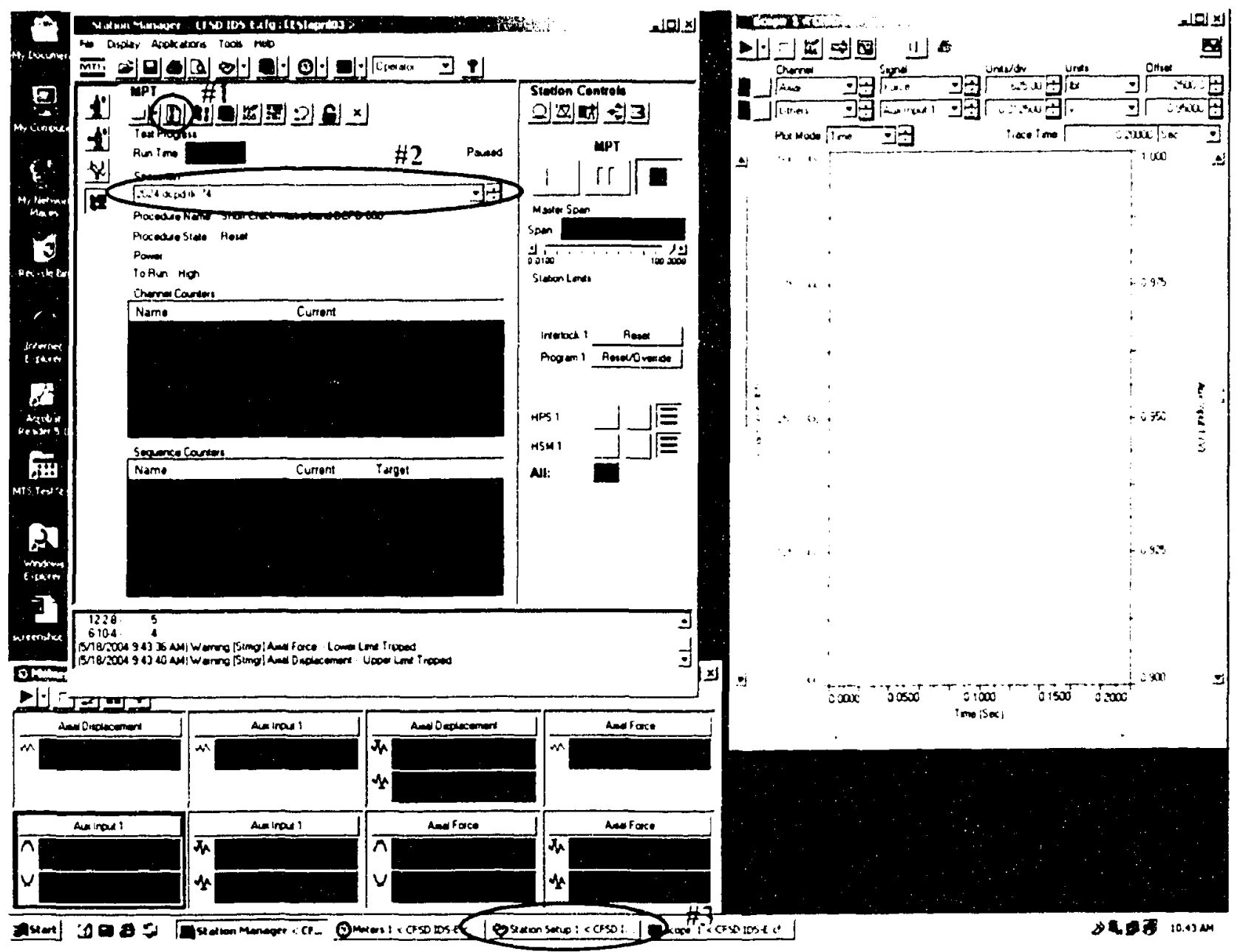

1) After the previous test is finished, record the cycles to failure in the log book, adjust the shims so that they are resting against the sample, take out the broken sample (as described in the fatigue sample loading procedure in Appendix A), and press the "New Sample" button (\#1).

2) Enter the new sample name (\#2).

3) Load the new sample into the axial load frame according to the procedure in Appendix A.

4) Attach the voltage and lead wires according to the diagram shown below. 


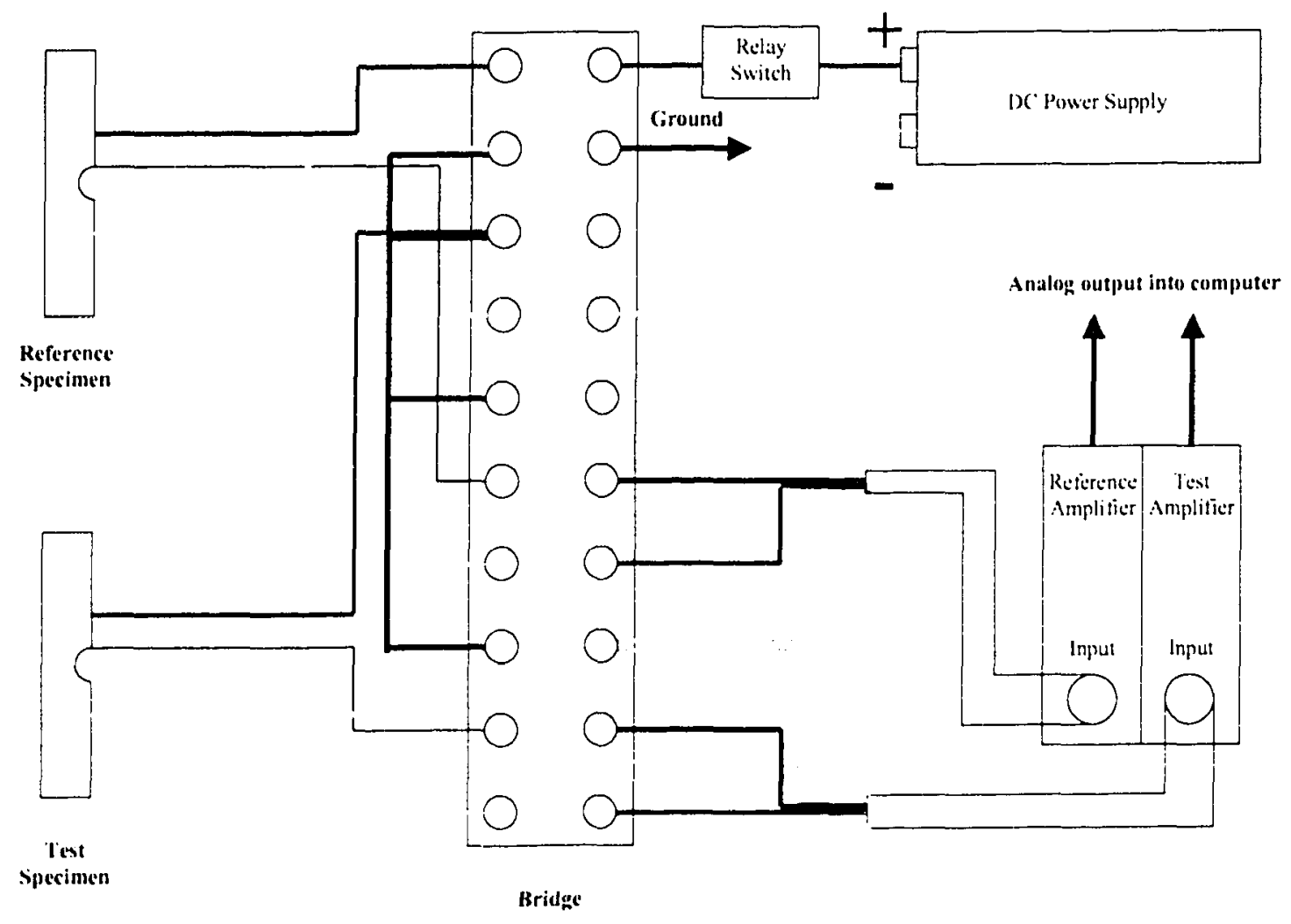

5) Before turning on the power supply, the upper voltage limit must first be disabled. To get to the voltage limit window, press the button in circle $\# 12$.

6) The upper limit can now be disabled by using the pull down window in circle \#13. Disabling the upper limit will remove any chance of surpassing that upper limit when the power supply is first turned on. The upper limit will be re-activated once the voltage readings have stabilized.

7) Minimize the station setup window. 


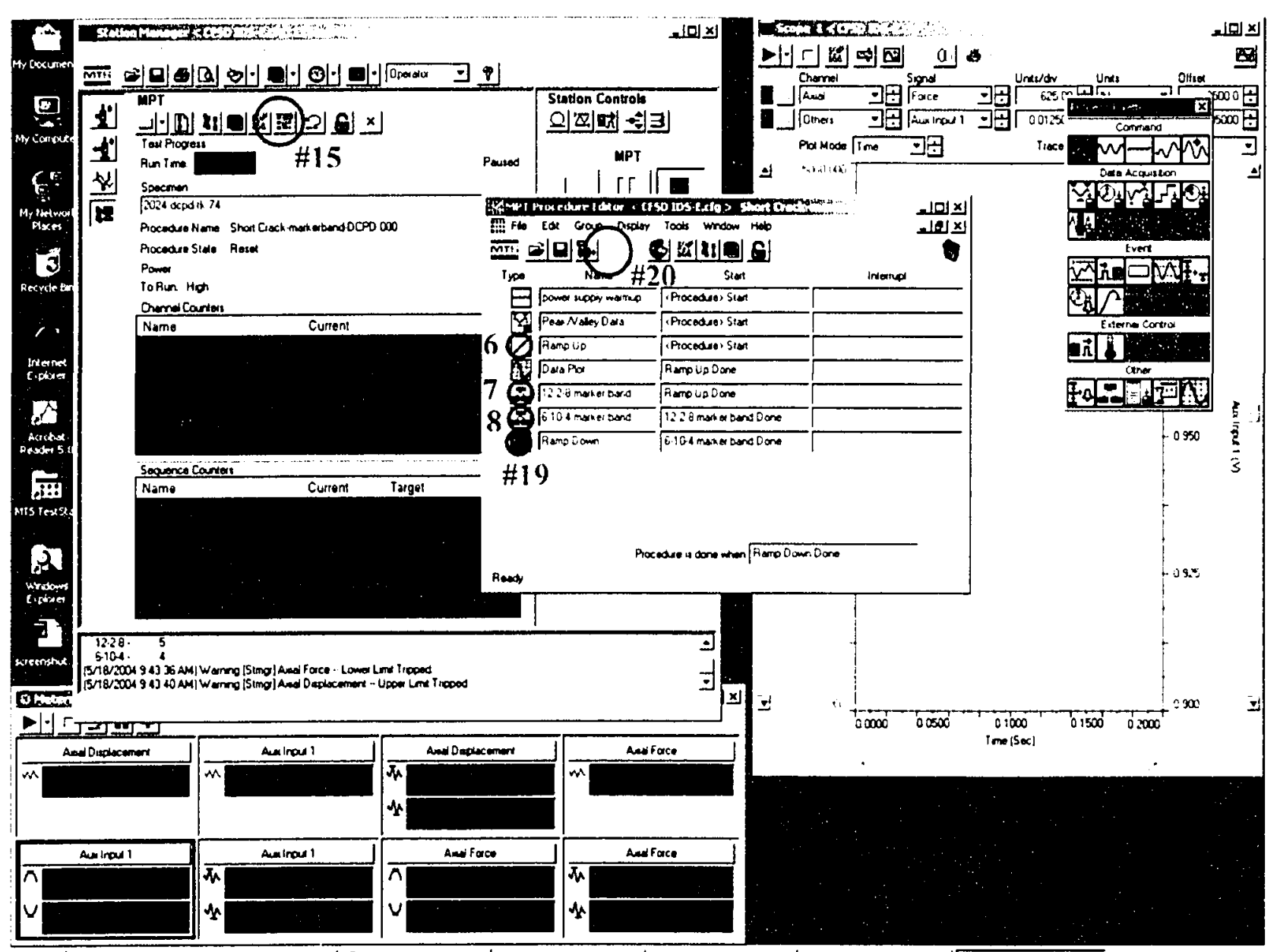

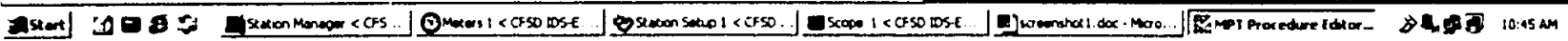

8) Any modifications to the fatigue testing procedure must be done in the MPT Procedure Editor. To access the procedure editor, press the button in circle $\# 15$.

9) If the lower fatigue load is changed, the ramp up load must also be changed to match the new lower fatigue load. This is accessed and changed by doubleclicking the button in circle $\$ 16$.

10) If the lower fatigue load has been changed, the ramp down load must also be changed to match that new load. This can be changed by double-clicking on circle \#19.

11) Any alterations to the first set of marker bands will need to be done by doubleclicking on circle $\# 17$.

12) Any alterations to the second set of marker bands will be accessed by doubleclicking on circle \#18. 


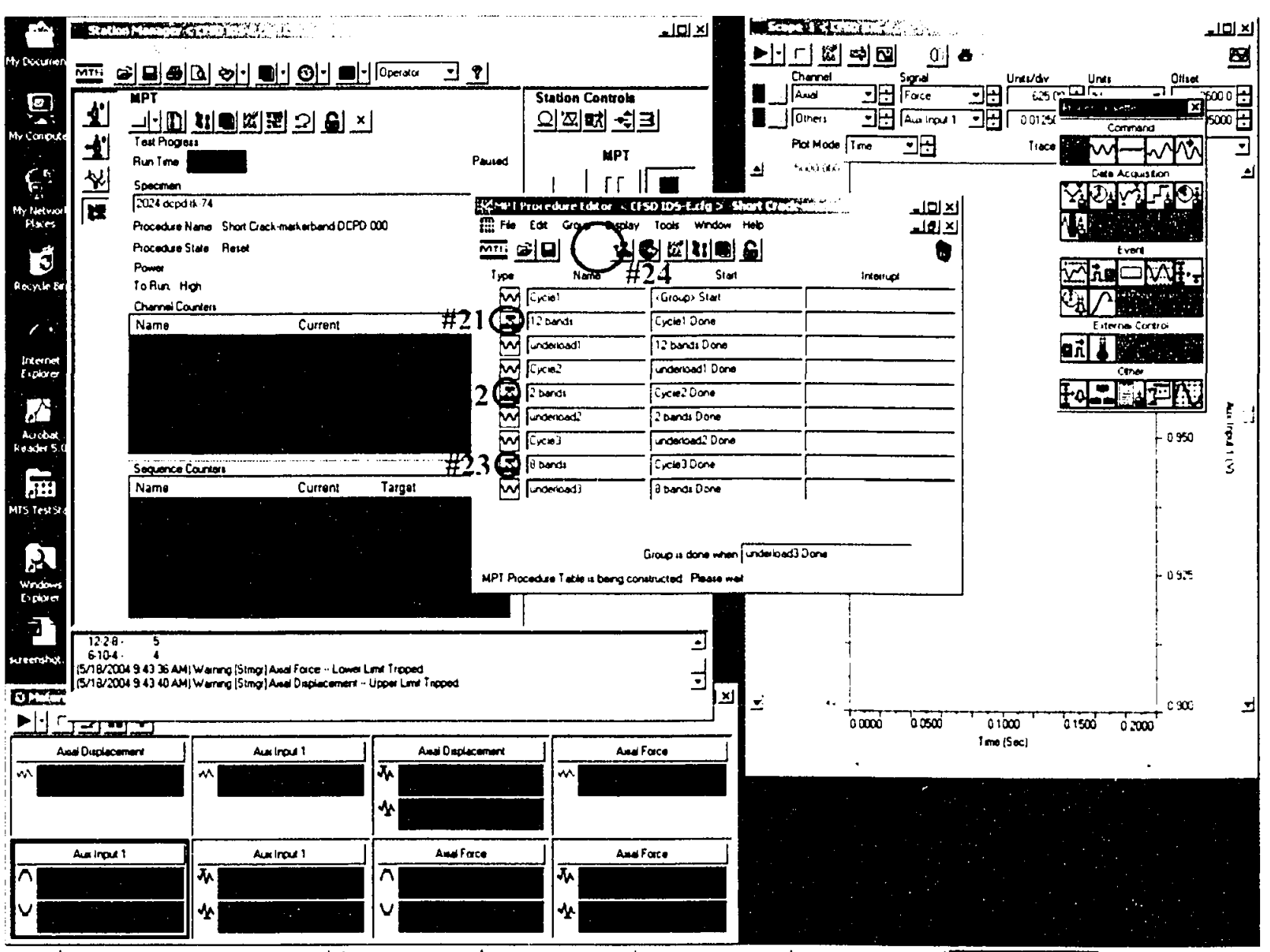

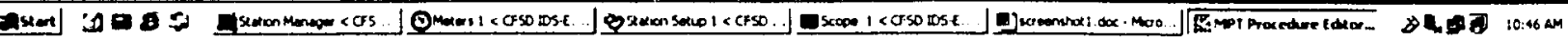

* Changing the upper and/or lower fatigue loads will require changes in all of the cyclic load commands.

13) To change the number of bands in the first group, double click on circle $\$ 20$ and enter the new number of bands.

14) To change the number of bands in the second group, double click on circle \#21 and enter the new number of bands.

15) To change the number of bands in the third group, double click on circle \#22 and enter the new number of bands.

16) The loads inside each group of marker bands must also be changed. To do this select circles $\# 21, \# 22$, and $\# 23$ one at a time and then click on circle $\# 24$ to expand the grouped procedure. 


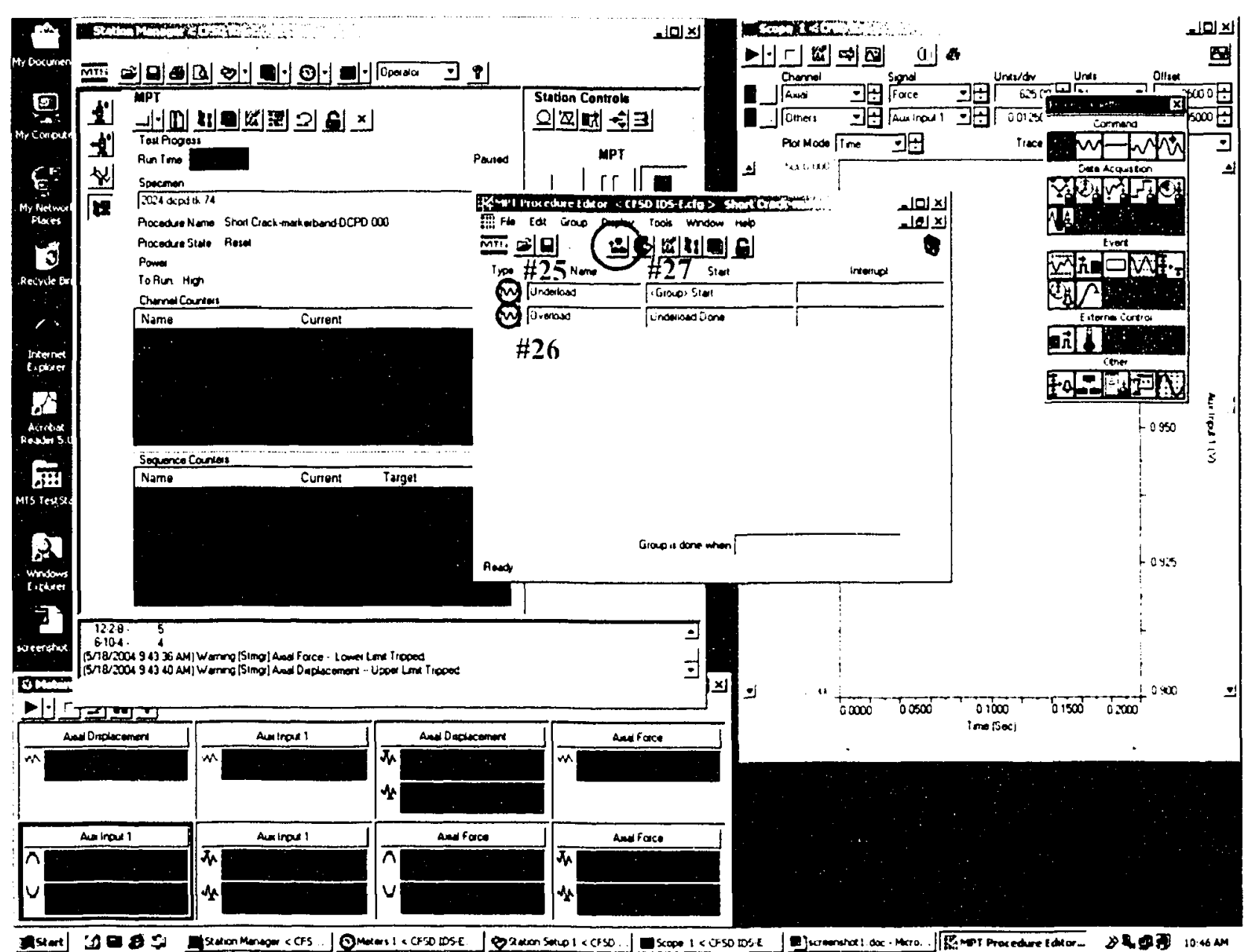

17) Double-click on circle \#25 to change the lower fatigue load.

18) Double-click on circle \#26 to change the upper fatigue load.

19) Click on circle \#27 to return to the previous window. Repeat for each group of marker bands. 


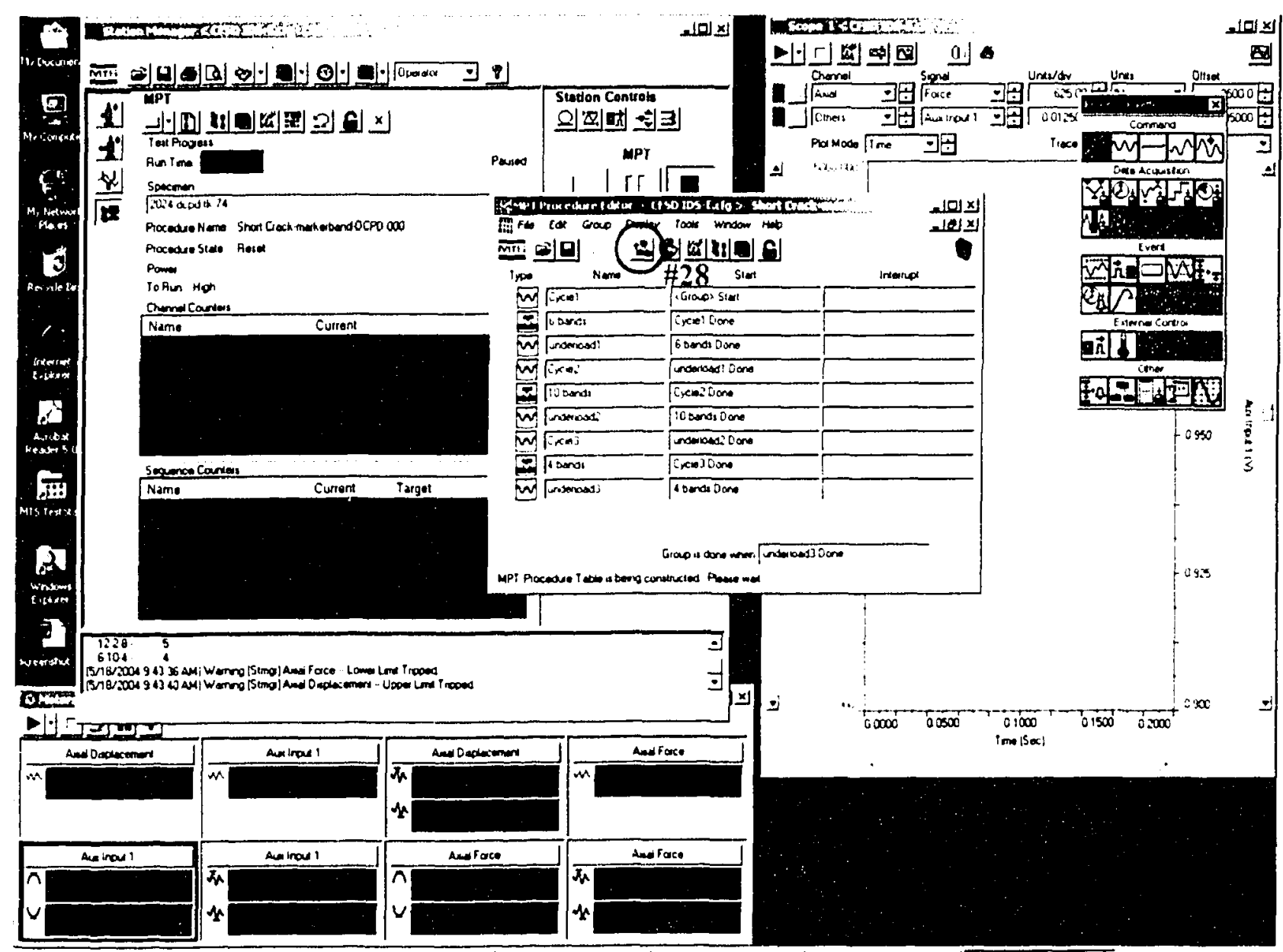

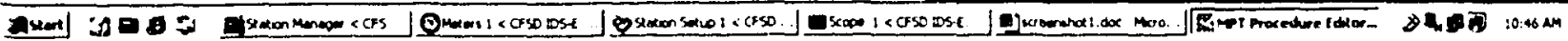

20) Once all the changes have been made, click on circle $\# 28$ to return to the main procedure window. 


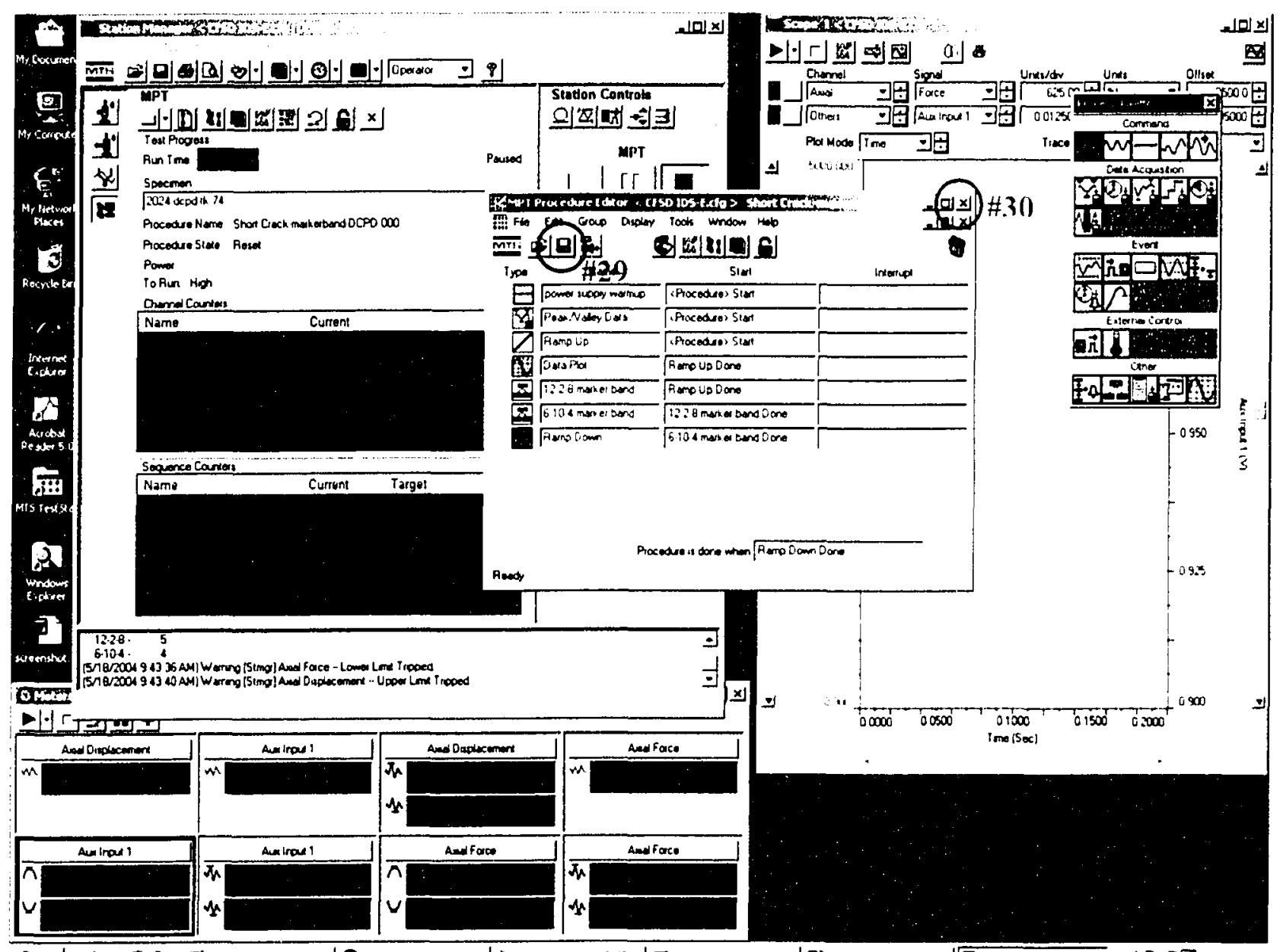

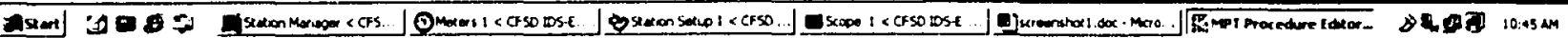

21) The procedure must be saved before it is recognized by the testing software. Click on circle \#29 to save the procedure.

22) Click on circle $\# 30$ to close the MPT Procedure Editor. 


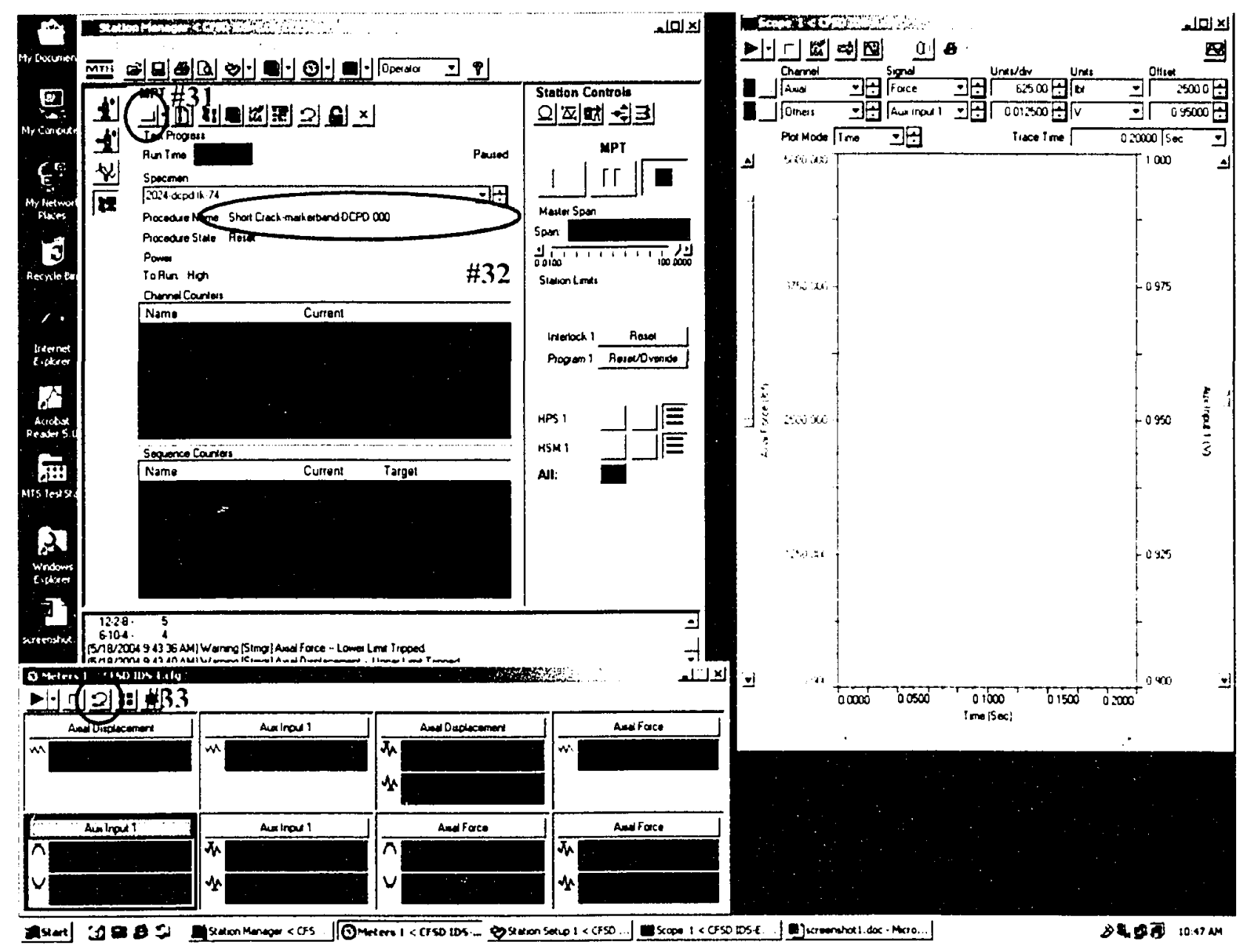

23) The modified procedure must be re-loaded before it used in the next fatigue test. Click on circle \#31 and select the correct procedure.

24) Check the procedure name within circle \#32 to make sure it is the correct procedure.

25) Reset the meter, by clicking on circle $\# 33$, so that the data shown will be for the current test. 


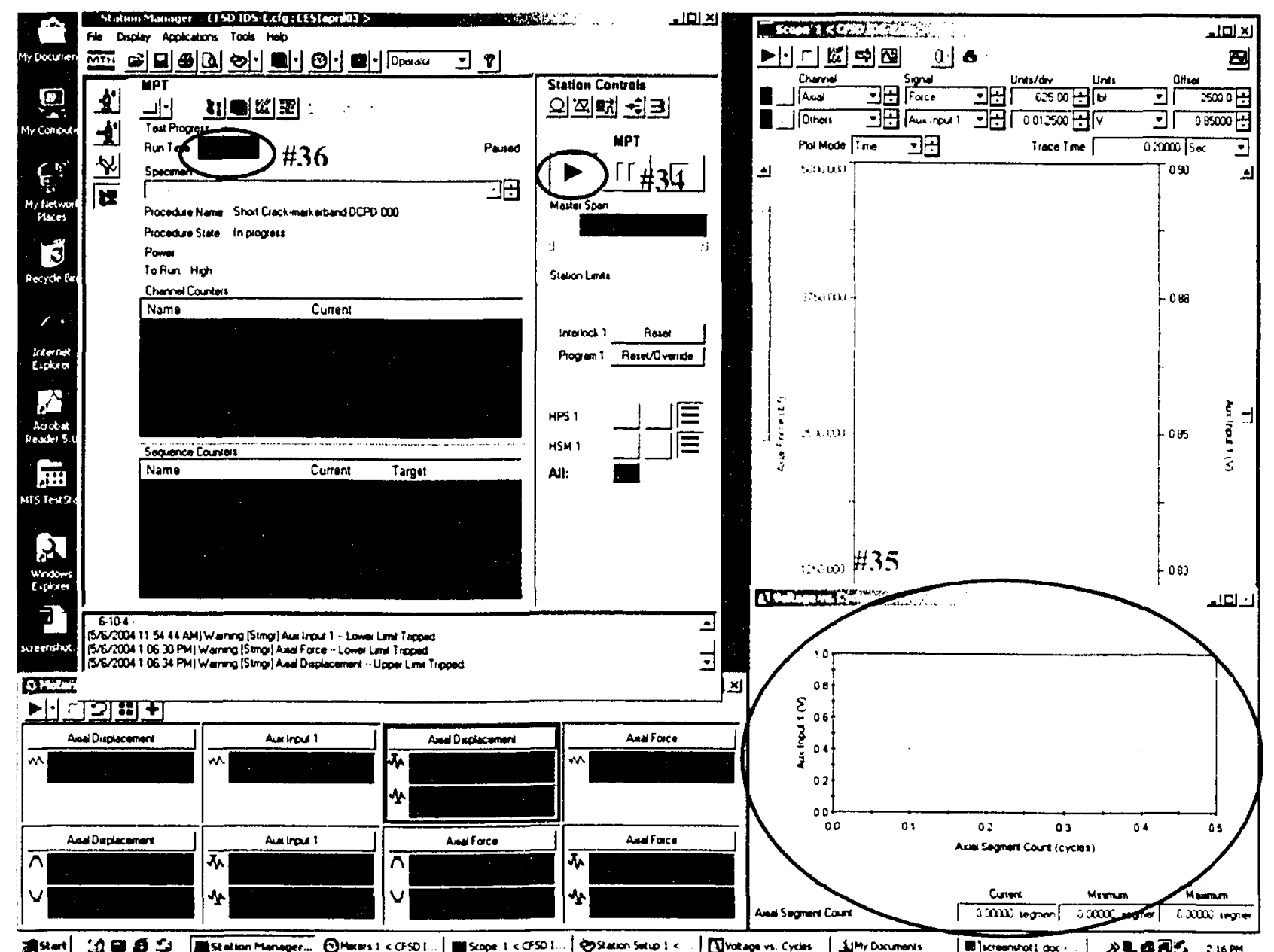

26) Start the test by pressing the button in circle $\# 34$. The procedure contains a two hour warm up time for the power supply.

27) A graph will appear on the bottom right of the screen as shown by circle $\# 35$ that will show the real-time voltage readings.

28) The timer in circle $\# 36$ shows the length of the test. 


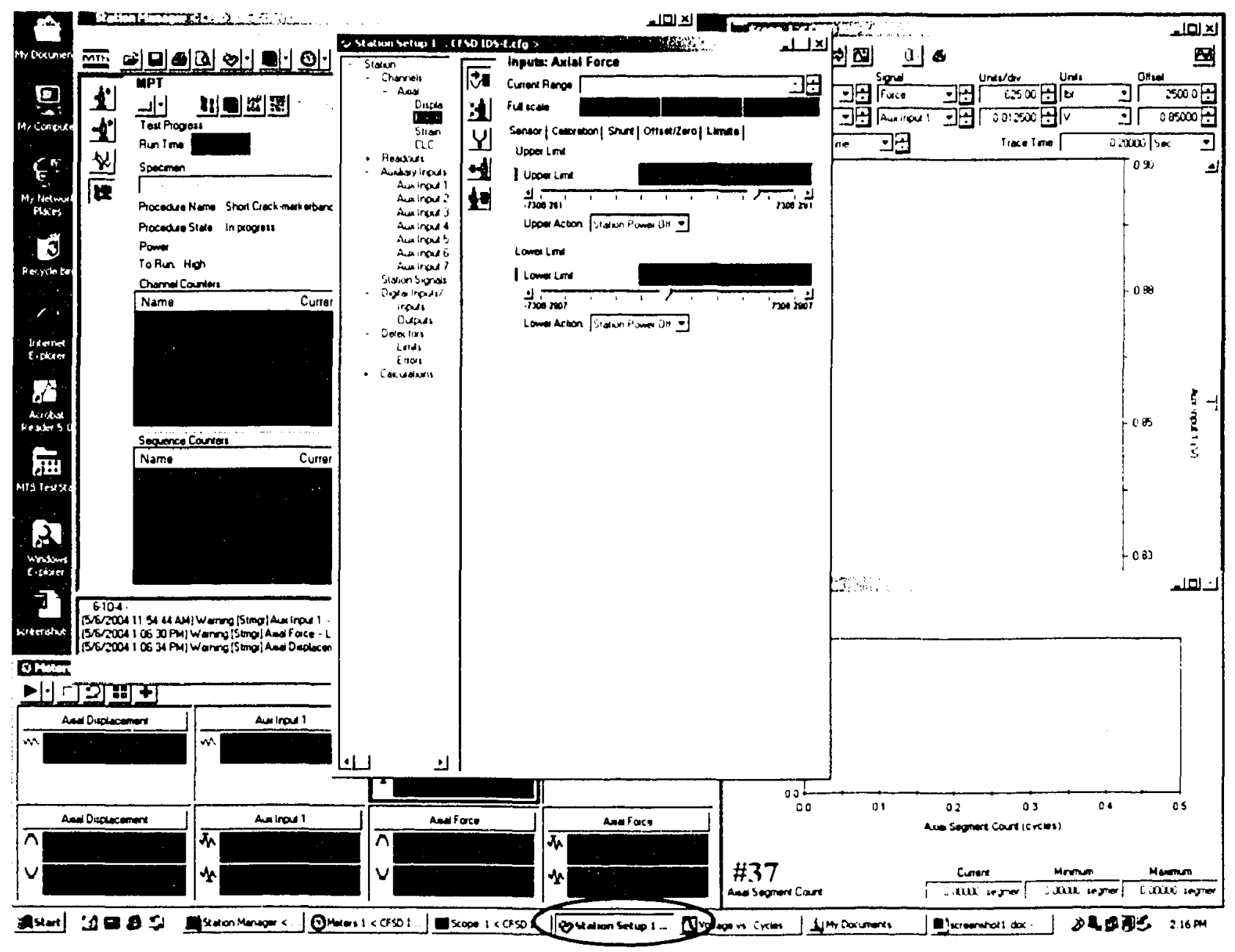

29) After the two hour warm-up time, the upper voltage limit must be set and activated. Return to the station setup by clicking on circle $\# 37$. 


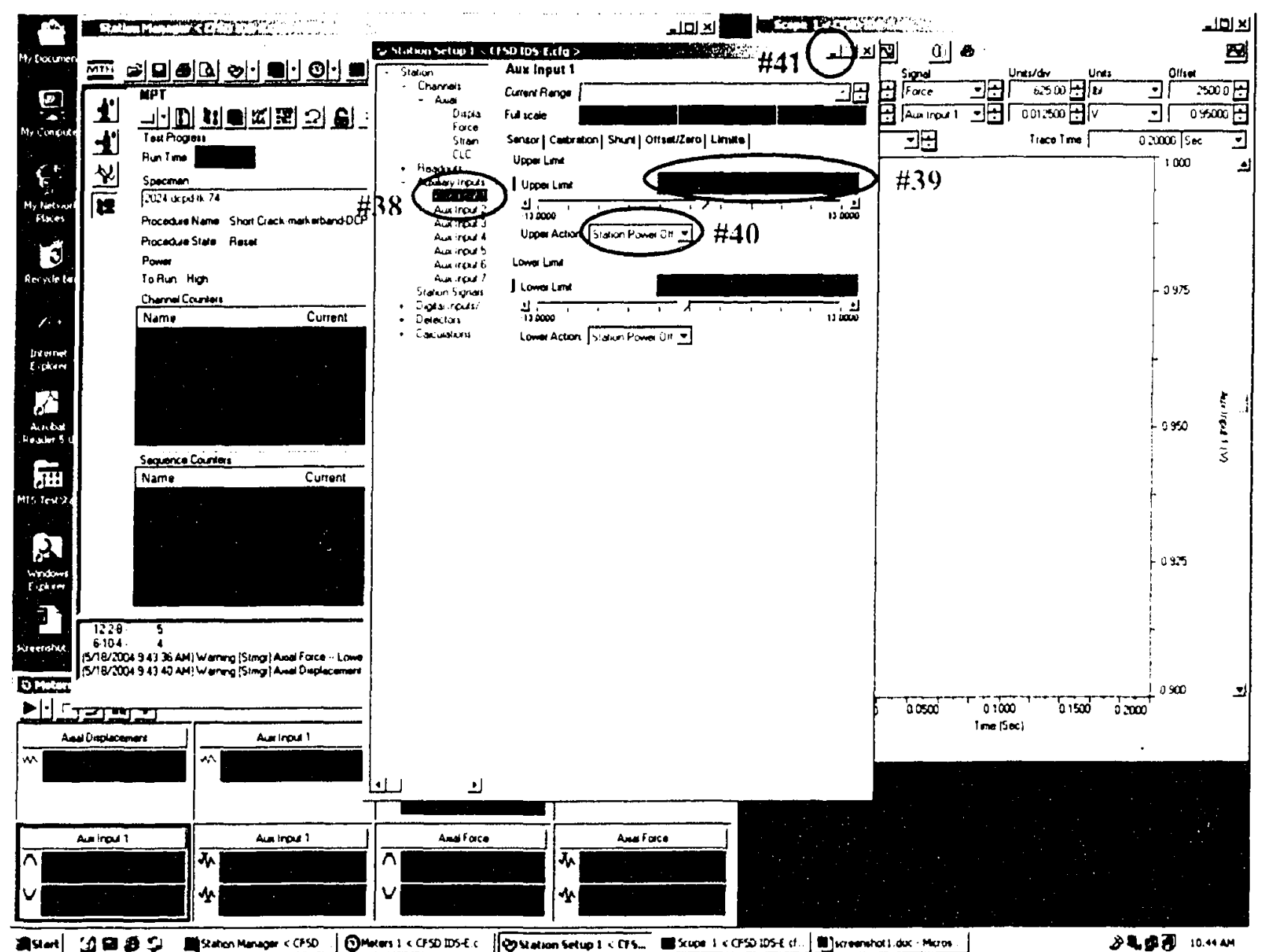

30) Select the channel that reads the voltage input signal (i.e. aux input 1 - circle $\# 38$ ).

31) In circle $\# 39$, type in the desired upper voltage limit and press "enter."

32) Activate the upper voltage limit by using the pull down menu highlighted in circle \#40 and selecting "Station Power Off."

33) Minimized the station setup window by clicking on circle $\# 41$. 


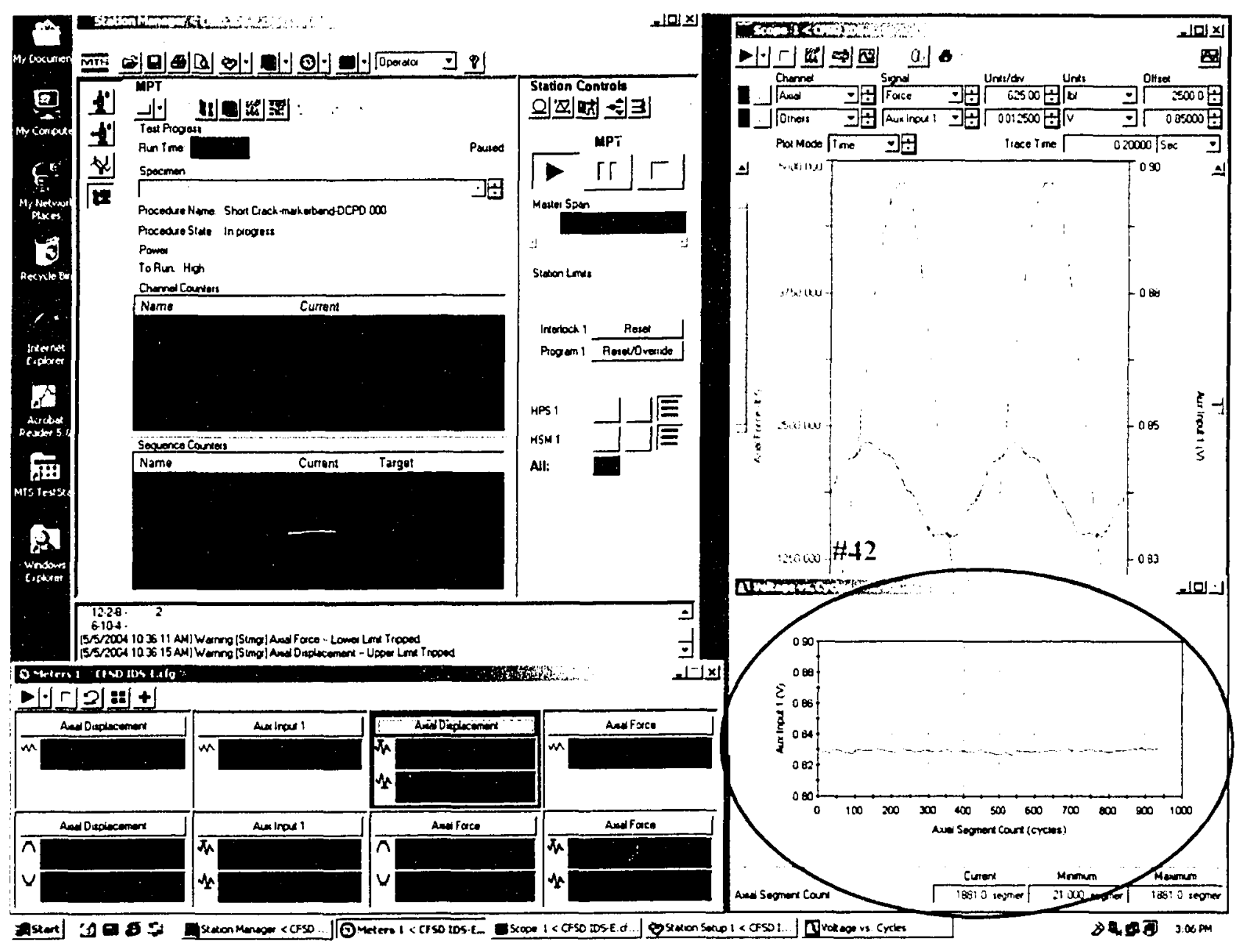

34) The voltage limits may require alterations during testing depending on the signal noise, voltage level, etc and this can be monitored by the real-time voltage drop curve plotted in circle $\# 42$. 